\title{
METODOS DE INCREMENTO \\ E BUSCA EM GRAFOS
}

\section{Celma de Oliveira Ribeiro}

Dissertação apresentada

ao

Instituto de Matemática e Estatistica

$d a$

Universidade de São Paulo

para obtenção do grau de

Mestre em Matemática Aplicada

Área de Concentração: Ciência de Computação

Orientador: Carlos Humes Júnior

- São Paulo, dezembro de 1987 - 
A minha mãe e

à minha avó 


\section{AGRADECIMENTOS}

À IBM do Brasil, pelo apoio financeiro concedido.

Ao Grupo Ultra, pelo apoio financeiro para minha participação no $\mathrm{X}$ CNMAC.

Ao orientador e amigo Carlos Humes Jr., sobretudo por ter me dado a consciencia de tudo a que tenho a aprender.

Aos professores Valdemar W. Setzer e Elza F. Gomide pelo estímulo dado para que eu iniciasse este trabalho.

A Luis Sérgio Tamer pela dedicação e inesgotável paciência com que me apoiou nos momentos mais difíceis da elaboração deste trabalho.

A Toni Takeshi pela grande dedicação na fase de datilografia.

A Ana Flora P. C. Humes pelo carinho com que me recebeu nas muitas noites em que trabalhei em sua casa.

Aos funcionários do IME-USP, pela sua constante ajuda; em particular ao Sérgio e ao Manoel, resposáveis pelo rápido trabalho de reprodução e encadernação.

A todos os amigos pelo constante apoio, decisivo para que este trabalho se completasse. 


\section{INTRODUÇÃO}

"O presente se desdobra a todo instante, em seu jorro mesmo, em dois jatos simétricos dos quais um recai no passado enquanto o outro se lança para o futuro"

Bergson

A motivação deste trabalho foi o estudo de dualidade subaditiva $e$ sua aplicação a problemas de programação linear definidos sobre grupos e a programção linear inteira. Esse conceito de dualidade tem sido bastante explorado na literatura não somente pela riqueza de sua estrutura, que garante a inexistência de "duality gaps", mas também por sua aplicaçã̃o na resolução destes problemas.

Dentro de uma outra abordagem,tais problemas podem ser resolvidos através de métodos para determinação de caminhos mínimos em grafos. 
Ao estudar esses métodos pudemos verificar que a subaditividade, também exercia um papel bastante importante no que se refere a sua complexidade computacional.

A partir daf, nosso trabalho passou a ser a busca de alguma relação entre algoritmos para a determinação de caminhos mínimos em grafos,em particular $A^{*}$, e algoritmos para a resolução do problema definido sobre grupos. Optamos pelo estudo deste problema e não pelo de programação linear devido a sua forte estrutura matemática.

Para que pudessemos uniformizar a notação utilizada, e não havendo uma referência básica comum aos métodos estudados, foi necessário que optassemos por um trabalho mais extenso, cuja organização é a que segue.

No capitulo 1 descrevemos o problema do caminho mínimo em termos de programação linear. Os capitulos 2, 3 e 4 se dedicam a algoritmos para a sua resolução, sendo que o capítulo 2 estuda os algoritmos de Dijlstre e Ford, 03 se dedica ao algoritmo $A$ e 0 quarto a, $A^{*}$. $O$ capítulo 5 apresenta o problema definido sobre grupos, sendo que no sexto é detalhada a aplicação de $A^{*}$ na sua resolução. 0 estudo de métodos de incremento é elaborado nos capítulos 7 e 8 , ficando claro neste último uma relação entre o método e $A^{*}$. 
Capítulo 1

\section{O Problema do caminho mais curto em grafos}

Um problema clássico em otimização é conhecido como "problema do caminho mais curto", e consiste na determinação de um caminho de custo mínimo entre um par fixado de nós de um grafo orientado, com custos fixos associados aos arcos.

Um grande número de autores vem estudando esse problema, de forma que é bastante extensa a literatura a seu respeito. Estudaremos nesse capitulo sua formulação em termos de programação linear, verificando suas principais características com relação a soluções básicas e dualidade. 


\section{1 - O PROBLEMA DO CAMINHO MINIMO}

O Problema que estaremos estudando parte da utilização de um grafo orientado com custos associados aos arcos, de forma que adotaremos as notaçōes e definições do apêndice 1 deste trabalho.

Durante esse capítulo e os que seguem admitiremos, a menos de menção explícita a contrário, que é satisfeita a seguinte hipótese.

\section{Hipótese Básica}

O grafo considerado $G \equiv(N, E)$ é orientado, conexo e tal que sua função de incidência $\xi_{G}(\cdot)$ é injetora, satisfazendo $\xi_{G}(e) \neq(i, i) \forall e \in E$, $\forall i \in N$.

Observe que segundo a definição de grafos adotada, a ocorrência de arcos "paralelos" é admitida, o que é excluído ao assumirmos $\xi_{G}(\cdot)$ injetora. Exemplificando, os grafos injetora)

$$
G_{1} \equiv\left(N_{1}, E_{1}\right) \operatorname{com} N_{1}=\{1,2\} E_{1}=\{(1,2),(1,2)\}\left(\xi_{G_{1}}(\cdot)\right. \text { não }
$$

$$
G_{2} \equiv\left(N_{2}, E_{2}\right) \operatorname{com} N_{1}=\{1,2\} E_{2}=\{(1,2)\}\left(\xi_{G_{2}}(\cdot) \text { injetora }\right)
$$

teriam a seguinte representação gráfica

$$
G_{1}:
$$

$G_{2}:$
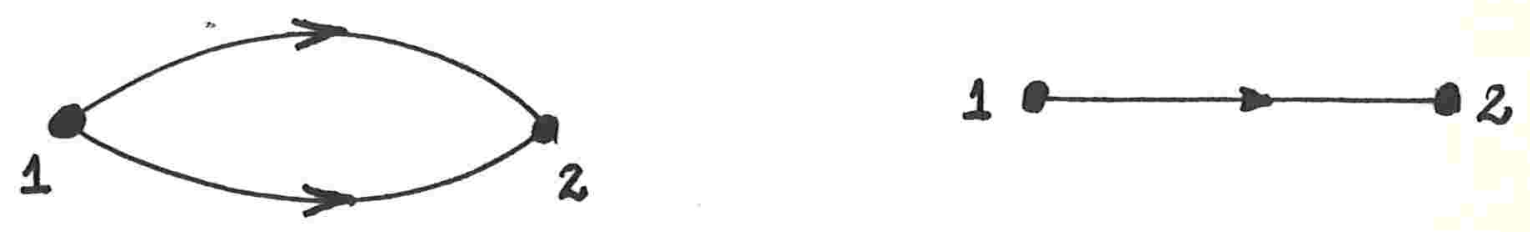

Além disso, note que ao admitirmos tal hipótese, teremos assegurado que o grafo $G \equiv(N, E)$ é finito se e somente se $\# N<\infty$. 
O problema que estaremos estudando é definido por:

\section{DEFINIÇÃO 1.1.1} (PCM)

Problema de determinação de caminho mínimo em grafos

Dado $G \equiv(N, E)$, um grafo orientado, $n_{0} \in N$ um nó fixado, $T \subset N$ um conjunto dado (de "alvos" a serem atingidos ) com $n_{0} \notin T$ e $c: E \rightarrow \overline{\mathbb{R}}$ a função custo associado aos arcos de $G$, determine, caso exista, um caminho $P_{\text {min }}=\left(n_{0}, i_{1}, i_{3}, \ldots, i_{r}\right)$ entre $n_{0}$ e $i_{r} \in T$, tal que seu custo $c\left(P_{\text {min }}\right)$ seja mínimo para todos os caminhos de $n_{0}$ a um nó "alvo". Ou ainda, encontre se existir, $P_{\min } \in P$ tal que $P_{\min } \in \arg \min \{c(P)\}$, com $\left.P=\left\{\omega \mid \omega \text { é um caminho entre } n_{0} \text { e } n_{8} \text { para algum } n_{8} \in T\right\}^{P}\right\}^{P}$

Para esse problema o grafo considerado não necessariamente deve ser finito ou possuir um conjunto finito de alvos. Porém, para que possamos formulá-lo de maneira simples como um problema de programação linear, assumimos a validade dessas condições como segue.

\section{HIPÓTESE 1.1.2}

O grafo $G \equiv(N, E)$ considerado é finito e o conjunto de elementos "alvo", $T$, possui um único nó, isto é, $T=\left\{n^{*}\right\}$ para algum $n^{*} \in N$. Por simplicidade, assumiremos que $N=\{1,2, \ldots, p\}, E=\{1,2, \ldots, q\}$ onde $p=\# N, q=\# E$ é ainda, $n_{0}=1$ e $n^{*}=p$.

A despeito de parecer restritiva, a condição \#T $=1$ pode ser assumida sem perda de generalidade, pois caso \#T $>1$ pode-se criar um grafo $G^{\prime} \equiv\left(N^{\prime}, E^{\prime}\right)$, adicionado-se um nó em $G \equiv(N, E)$, isto é, $N^{\prime}=N \cup\{\hat{n}\}$ e tomando-se $E^{\prime}=E \cup\{(i, \hat{n}) \mid i \in T\}$, associando às arestas adicionadas um custo nulo. Assim obtem-se um novo grafo para o qual o problema associado é equivalente ao original.

É importante salientar que ao assumir que os grafos estudados são finitos estamos admitindo implicitamente que o problema satisfaz inf $f_{p \in P}$ $c(p) \in \mathbb{R} \cup\{+\infty\}$ ( $P$ conforme DEFINIÇÃO 1.1.1) pois segundo a definição 
adotada, caminhos não contém circuitos, o que garante a existência de um número finito de caminhos. Para que tenhamos maior flexibilidade em nosso trabalho podemos admitir passeios, ao invés de caminhos, na definição do conjunto $P$, restringindo as soluçóes ótimas do problema a serem caminhos.

\section{DEFINIÇÄO 1.1.3}

Para o problema de determinação de caminho mínimo em um grafo $G \equiv(N, E)$, diremos que

i) o problema é viável se existe pelo menos um passeio entre 1 e p

ii) o problema é ilimitado se existe uma sequêencia $\left\{P^{k}\right\}_{k \in \mathbb{N}}$ de passeios entre 1 e $p$ tais que

$$
\lim _{k \rightarrow \infty} c\left(P^{k}\right)=-\infty
$$

A hipótese de finitude do grafo é bastante restritiva, excluindo de nosso estudo uma considerável quantidade de problemas. Entretanto, para que problemas definidos em grafos não finitos possuam solução ótima, é necessário que:

i) 0 alvo seja atingível a partir da origem por um passeio, o qual, segundo definição adotada é finito.

ii) $\exists p^{k} \in \underset{n \in p}{\arg \operatorname{mix}}(p)$, o que näo ocorre no seguinte exemplo:

$$
G \equiv(N, E) \quad N=\mathbb{N} \cup\{+\infty\}
$$

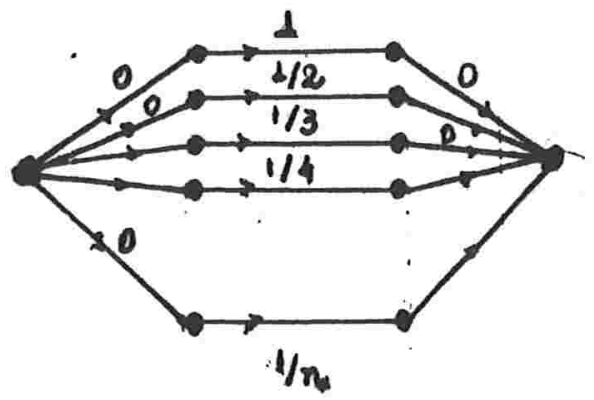

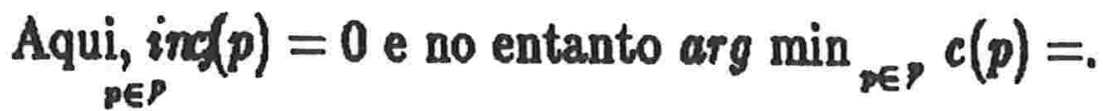


Ao estudarmos métodos para resolução do problema é usual admitirmos hipóteses que de certa forma correspondam a assumirmos uma certa "finitude" do horizonte de trabalho.

Grosseiramente, como métodos para determinação de caminhos mínimos devem de certa forma, "pesquisar" todos os caminhos que eventualmente conduzam à solução do problema, é natural admitirmos, por exemplo, que o número de caminhos cujo custo não supera o valor ótimo do problema seja finito e que o grafo seja localmente finito (isto é, $\forall j \in$ $N \# \operatorname{suc}(j)<+\infty)$.

Antes de passarmos à formulação do problema propriamente dito, estudaremos a sua relação com espaços vetorais. A linha que seguiremos baseia-se no trabalho de Murty [Mu 67].

\section{DEFINIÇÃO 1.1.4}

Diremos que uma função $y: E \rightarrow \mathbf{R}$ é uma circulação se

$$
\forall i \in N, \sum_{j:(i, j) \in E} y(i, j)-\sum_{k:(k, i) \in E} y(k, i)=0
$$

Ou seja $y(i, j)$ pode ser interpretado como uma quantidade de corrente elétrica que passa entre dois pontos $i \in N$ e $j \in N$,para a qual é satisfeita a lei de Kirchhof, qual seja, a soma algébrica das correntes que passam por um nó é nula (isto é, a quantidade de carga elétrica que "entra" no nó é a mesma que "sai").

\section{DEFINIÇÄO 1.1 .5}

Dizemos que uma função $z: E \rightarrow \mathbb{R}$ é uma função diferença de potencial se para alguma função $w: N \rightarrow \mathbb{R}, z(\cdot)$ satisfaz

$$
z(i, j)=\omega(i)-\omega(j), \forall(i, j) \in E
$$

Para essas funçő̉es tem-se

TEOREMA 1.1.6 
Os conjuntos $W_{C}=\{y: E \rightarrow \mathbb{R} \mid \mathrm{y}(\cdot)$ é uma circulação $\}$ e $W_{P}=\{z: E \rightarrow \mathbb{R} \mid z(\cdot)$ é função diferença de potencial $\}$ são espaços vetoriais sobre $\mathbb{R}$.

\section{Demostração}

Trivial, observando-se que a soma de circulações (potenciais) bem como a multiplicação por escalar fornecem circulações (funções diferença de potencial)

O espaço $W_{C}$ é comumente chamado Espaço dos Ciclos pois pode-se verificar que aos elementos de $W_{C}$ estão associados semi-circuitos no grafo considerado (ou equivalentemente, ciclos no grafo não orientado induzido por $G)$.

Para que verifiquemos essa relação consideraremos a "Matriz de Incidencia do Grafo $G \equiv(N, E)$ definida por

$$
\begin{gathered}
M=\left(m_{i}(a)\right) \in \mathbb{R}^{\# N \times \# E} \\
m_{i}(a)=\left\{\begin{array}{cl}
+1 & \text { se } a=(i, j) \\
-1 & \text { se } a=(j, i) \\
0 & \text { caso contrário }
\end{array} \quad \forall i \in N \quad \forall a \in E\right.
\end{gathered}
$$

Tendo admitido finitude no grafo considerado, para cada função $f: E \rightarrow \mathbb{R},(E=\{1,2, \ldots, q\}$ o conjunto de arcos do grafo $)$, podemos associar um vetor de $\mathbb{R}^{q}$ que define $f(\cdot)$, a saber, $f^{v}=(f(1), f(2), \ldots, f(q))^{t}$. Em particular, circulaçóes no grafo podem ser considerados vetores $y \in \mathbb{R}^{q}$, - que nos permitirá, posteriormente, associar dependência linear de colunas de $M$ com essas circulaçóes.

\section{TEOREMA 1.1.7}

Dado um grafo orientado $G \equiv(N, E)$ e a correspondente matrix $M$ , (cujas linhas denotaremos por $M_{j}, \forall j \in N$ ) tem-se que $W_{p}=\left[\left\{M_{j}\right\}_{j \in N}\right]$, ou seja, $W_{p}$ é o espaço gerado pelas linhas da matriz de incidência de $G$. 
Capítulo 1: O Problema do caminho mais curto em grafos

Demonstração

i) $W_{p} \subset\left[\left\{M_{j}\right\}_{j \in N}\right]$

Basta observar que $\forall p() \in W_{p}, \exists \omega: E \rightarrow \mathbb{R} \mid p(i, j)=\omega(i)-$ $\omega(j) \forall(i, j) \in E$ e portanto $\forall(i, j) \in E$,

$$
\begin{aligned}
& p(i, j)=\omega(i) * 1+\omega(j) *(-1)+\sum_{k \in N \backslash\{(i, j)\}} \omega(k) * 0= \\
& \begin{aligned}
=\omega(i) * m_{i}(i, j)+\omega(j) * m_{j}(i, j)+\sum_{k \in N \backslash\{(i, j)\}} \omega(k) * m_{k}(i, j)= \\
=\sum_{k \in N} \omega(k) * m_{k}(i, j)
\end{aligned} \\
& \text { ii) }\left\{\left\{M_{j}\right\}_{j \in N}\right] \subset \omega_{p}
\end{aligned}
$$

Claramente qualquer combinação linear de linhas de $M$ corresponde a uma função potencial e segue a afirmação.

O espaço vetorial gerado pelas circulaçöes $\left(W_{C}\right)$ por uma vez mantém uma estreita relação com $\omega_{p}$.

\section{TEOREMA 1.1.8}

Sendo $\langle\because, \cdot\rangle$ o produto interno usual de $\mathbb{R}^{\# E}$, e identificando-se as funções $f: E \rightarrow \mathbb{R}$ com $\mathbb{R}^{\# E}$, tem-se que $W_{C}$ é o complemento ortogonal de $W_{p}$, ou seja, $W_{C}=W_{p}^{\perp}$

Demonstração

i) $W_{C} \subset W_{p}^{\perp}$ 
Capítulo 1: O Problema do caminho mais curto em grafos

Como por definição $y(\cdot) \in W_{C}$ se e só se

$$
\forall i \in N \sum_{j:(i, j) \in E} y(i, j)-\sum_{k:(k, i) \in E} y(k, i)=0 \text {, segue que }
$$

$$
\forall i \in N \sum_{j:(i, j) \in E} y(i, j)+\sum_{k:(i, k) \in E}(-1) y(k, i)+\sum_{(r, 1) \in E_{i}} 0 * y(r, i)=0
$$

onde $E_{i}^{*}=E \backslash\{(j, k) \subset E \mid j=$ oou $k=i\}$

Assim, $\forall i \in N$

$$
\begin{gathered}
\sum_{j:(i, j) \in E} m_{i}(i, j) y(i, j)+\sum_{k:(k, i) \in E} m_{i}(k, i) y(k, i)+\sum_{(r, 1) \in E_{i}} m_{i}(r, 1) y(r, 1)= \\
\sum_{a \in E} m_{i}(a) y(a)=0
\end{gathered}
$$

Ou seja, $y(\cdot)$ é ortogonal às linhas da matriz de incidência do grafo e portanto $W_{C} \subset W_{p}^{\perp}$

ii) $W_{p}^{\perp} \subset W_{C}$

Seja $p \in W_{p}^{\perp}$ então como $W_{p}=\left[\left\{M_{j}\right\}_{j \in N}\right]$ pelo TEOREMA 1.1.7,

$\left\langle p, M_{i}\right\rangle=0 \quad \forall i \in N$,ou seja,

$$
\forall i \in N \sum_{a \in \mathbb{E}} p(a) M_{i}(a)=0
$$

(pla corresponde à componente associada ao arco $a$ no vetor $p$ )

Como $M_{i}(a) \neq 0$ se e somente se $a \in E_{i}=\{a=(j, k) \in E \mid j=i$ ou $k=i\}$ vem que

$$
\forall i \in N
$$


Capítulo 1: O Problema do caminho mais curto em grafos

$$
\begin{gathered}
0=\sum_{a \in E} p(a) M_{i}(a) \\
=\sum_{a \in E_{i}} p(a) M_{i}(a) \\
=\sum_{j:(i, j) \in E} p(i, j) M_{i}(i, j)+\sum_{k:(k, i) \in E} p(k, i) M_{i}(k, i) \\
=\sum_{j:(i, j) \in E} p(i, j)+\sum_{k:(k, i) \in E} p(k, i)
\end{gathered}
$$

Assim $p$ é uma circulação e portanto $W_{p}^{\perp} C$

De (i) e (ii) segue a afirmação.

Nosso objetivo com esse estudo sobre espaços vetoriais é tentar associar propriedades como independencia linear a propriedades de grafos, como por exemplo, ser acíclico pois com isso podemos associar, caminhos a elementos dos espaços vetoriais.

\section{LEMA 1.1.9}

Seja y $E \rightarrow \mathbb{R}$ uma funçäo e considere o suporte de y definido por $\operatorname{supp}(y)=\{a \in E(a) \neq 0\}$

Então se y(*) é circulação não nula, $\forall i \in N$, ou $\exists\left(k_{1}, k_{2}\right) \in N \times N$ tal que $\left(i, k_{1}\right) \in \operatorname{supp}(y)$ e $\left(k_{2}, i\right) \in \operatorname{supp}(y)$ ou $\forall k \in N(i, k) / \operatorname{supp}(f)$ e $(k, i) / \operatorname{supp}(f)$, isto é, os "nós" de supp $(y)(\neq \emptyset)$ possuem grau maior que um.

1)

Portanto sabemos que supp(y) contém um semi-circuito. (cf.Apendice

Vamos então considerar submatrizes de $M$, a matriz de incidência do grafo. Para um dado subconjunto $S \subset E$, denotaremos por $M / S$ à matriz formada pelas colunas de $M$ correspondentes aos arcos $a \in S$. Com essa notação vale então o seguinte resultado: 


\section{TEOREMA 1.1.10}

Seja $S$ um subconjunto qualquer de $E$, e $M / S \in \mathbb{R}^{\# N \times \# S}$ formada pelas colunas da matriz de incidência do grafo que correspondem aos elementos de $S$.

Então as colunas de $M / S$ são linearmente independentes se e só se $S$ não contém semi-circuitos.

\section{Demonstração}

Denotando por $M(a)$ à coluna de $M / S$ correspondente ao arco $a \in S$, tem-se

$$
\begin{gathered}
\{M(a)\}_{a \in S} \text { LD } \\
\Leftrightarrow \exists \alpha: S \rightarrow \mathbb{R} \mid \sum_{\mathrm{a} \in \mathrm{S}} \alpha(\mathrm{a}) \mathrm{M}(\mathrm{a})=0, \alpha(\overline{\mathrm{a}}) \neq 0
\end{gathered}
$$

para algum $\bar{a} \in S$

Assim, $G$,

i) Suponha que $\{M(a)\}_{a \in S}$ é LD e considere a função definida em

$$
\begin{gathered}
\beta: E \rightarrow \mathbb{R} \\
\beta(a)= \begin{cases}\alpha(a) & a \in S \\
0 & a \notin S\end{cases}
\end{gathered}
$$

Claramente $\beta(\cdot)$ assim definida é uma circulação não nula $(\beta(\bar{a}) \neq$ $0)$. Consequentemente $\operatorname{supp}\{\beta\} \neq \emptyset$ e do LEMA 1.1.9 $S$ contém um semicircuito. Logo, se $S$ não contém semi-circuitos então $\{M(a)\}_{a \in S}$ é $L I$.

i) Reciprocamente, se $C$ é um semi-circuito de $S$, tome $C^{*}$ o circuito definido a partir de $C$, onde $\forall(i, j) \in C^{*} \quad(i, j) \in C$ ou $(j, i) \in C$ (isto é, "muda-se" a orientação de $C$ de forma a se obter um circuito)

Tomemos então $y: E \rightarrow \mathbb{R}$ definida por

$$
y(i, j)= \begin{cases}1 & \text { se }(i, j) \in C \cap C^{*} \\ -1 & \text { se }(i, j) \in C \backslash C^{*} \\ 0 & \text { caso contrário }\end{cases}
$$


É de fácil verificação que $y(\cdot)$ assim definida é uma circulação em $G$, näo nula. Assim,

$$
\begin{aligned}
& \sum_{a \in S} y(a) M(a)=\sum_{a \in C} y(a) M(a)+\sum_{a \in E \backslash S} y(a) M(a) \\
= & \sum_{a \in E} y(a) M(a)=0 \text { e portanto }\{M(a)\}_{a \in S} \text { LD }
\end{aligned}
$$

De (i) e (ii) segue a tese.

Retornando ao nosso problema original, pretendemos determinar caminhos entre pares de nós do grafo. Como caminhos não possuem semicircuitos é natural buscar a relação entre nosso problema e a determinação de funções $x(\cdot)$ definidas sobre $E$, que mantenham a independência linear de $\{M(a)\}_{a \in \operatorname{supp}\{x\}}$.

Vamos entao estudar o seguinte problema.

\section{DEFINIÇÃO 1.1.11}

Dado $G \equiv(N, E)$ um grafo safisfazendo a HIPÓTESE 1.1.2, sejam $M$ sua matriz de incidência, $1 \in N$ o nó origem e $p \in N$ o nó alvo. Definiremos o problema do caminho mais curto na forma canônica (PMINC) por:

Encontre, se existir, $x^{k} \in \arg \min _{x \in X_{\min }}\{\langle c, x\rangle\} X^{1 p}=X_{\min }=$ $\left\{x \in \mathbb{R}^{\# E} \mid M x=e^{1}-e^{p} \quad x 0\right\}$ onde $\forall j \in I_{p}, E^{j}$ denota $0 j$-ésimo vetor da base canônica de $\mathbb{R}^{\mathrm{n}}$, isto é,

$$
\forall k \in\{1,2, \ldots, n\} \quad e_{k}^{j}= \begin{cases}1 & \text { se } k=j \\ 0 & \text { caso contrário }\end{cases}
$$

Sendo (PMINC) um problema linear sabemos resolvido pelos métodos usuais tais como o simplex. Portanto se as soluções dos problemas (PMINC) e de determinação do caminho mínimoforem equivalentes, já temos um primeiro método de resolução do problema original. 
Seja $p_{k_{1} k_{3}}$ um caminho entre dois nós $k_{1} \in N$ e $k_{2} \in N$ com custo $c^{*}=c\left(P_{k_{1} k_{2}}\right.$. Então $\exists x^{*} \in X^{k_{1} k_{2}}$, onde, $X^{k_{1} k_{2}}=\left\{x \in \mathbb{R}^{\# \mathbb{E}} \mid M x=\right.$ $\left.e^{k_{1}}-e^{k_{2}} x 0\right\}$, tal que $\left\langle c, x^{*}\right\rangle=c^{*}$.

Demonstração

Considere $x^{*} \in \mathbb{R}^{\# \mathbb{E}}$ definido por

$$
x_{i j}^{*}= \begin{cases}1 & \text { se }(i, j) \in P_{k_{1} k_{2}} \\ 0 & \text { caso contrário }\end{cases}
$$

Claramente $x^{*} 0$. Alerdisso,

$$
\begin{aligned}
M x^{*}= & \sum_{(i, j) \in E} M(i, j) x_{i, j}^{*}=\sum_{(i, j) \in P_{k_{1} k_{2}}} M(i, j) x_{i j}^{*}= \\
& \sum_{(i, j) \in P_{k_{1} k_{2}}} M(i, j)=\sum_{(i, j) \in P_{k_{1} k_{2}}}\left(e^{i}-e^{j}\right)
\end{aligned}
$$

Mas $\forall j i n N \backslash\left\{k_{1}, k_{2}\right\}$ tal que $j$ é um nó do caminho $P_{k_{1} k_{2}}, \exists !\left(j_{1}, j_{2}\right)$ $\in N \times N$ tal que $(j 1, j) \in P_{k_{1} k_{2}}$ e $\left(j, j_{2}\right) \in P_{k_{1} k_{3}}$

Assim :

$$
\begin{gathered}
M x^{*}=e^{k_{1}}-e^{k_{3}}+\sum_{\substack{(i, j) \in P_{k_{1} k_{2}} \\
i \neq k_{1} \\
j \neq k_{2}}}\left(e^{i}-e^{j}\right)= \\
=e^{k_{1}}-e^{k_{3}}+\sum_{\substack{(i, j) \in P_{k_{1}} k_{2} \\
i \neq k_{1}}}\left(e^{i}-e^{i}\right)=e^{k_{1}}-e^{k_{2}}
\end{gathered}
$$

e consequentemente $x^{*} \in X^{k_{1} k_{2}}$. Claramente por construção $C^{*}=\langle c, x *\rangle$.

\section{COROLARIO 1.1.13}

Qualquer que seja o caminho $P$ entre 1 e $p \exists x^{*} \in X_{\min }$ tal que $\left\langle c, x^{*}\right\rangle=c(P)$. 
Ao contrário do que possa parecer, os elementos viáveis nos conjuntos $X^{k_{1} k_{2}}$ não necessariamente correspondem a caminhos no grafo considerado. Esse é o caso por exemplo do grafo $G \equiv(N, E)$, dado por

$$
\begin{aligned}
& N \equiv\{1,2,3\} \\
& E=\left\{e_{1}, e_{2}, e_{3}, e_{4}\right\}
\end{aligned}
$$

Assumindo-se que os nós origem e destino sejam respectivamente 1 e 3 , tem-se que $x^{*}=(1,0,1,1)^{t}$ é viável em $X^{1,3}$. Associando-se a cada elemento $x_{e}^{*} \neq 0$ o correspondente arco em $\mathrm{G}$, o subgrafo então obtido é

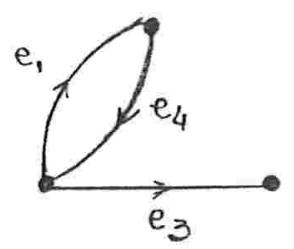

que claramente näo corresponde a um caminho entre 1 e 3 pois possui um circuito.

Um outro exemplo interessante nesse mesmo grafo é obtido ao considerarmos o elemento $x^{*}=(0.5,0.5,0,0.5)^{t}$ viável em $X^{1,3}$. Construindose o subgrafo correspondente a $x^{*}$ vem:

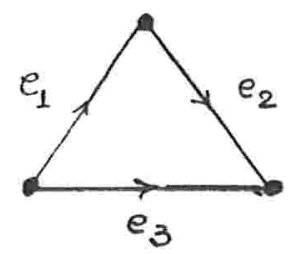

e que novamente não corresponde a um caminho devido à existência de semi-circuitos.

Fica claro nos dois casos que apesar de näo haver correspondência entre os elementos $x^{*} \in X^{1,3}$ apresentados e caminhos entre os nós origem e destino, o nó 3 é atingivel a partir de 1 por um semi-passeio, o que sugere que a elementos viáveis de $X^{k_{1}, k_{2}}, \forall\left(k_{1}, k_{2}\right) \in N \times N$, estejam associados de 
alguma forma, semi-passeios entre os nós $k_{1}$ e $k_{2}$, ou se possível, caminhos entre eles.

A verificação de resultados nessa linha se utiliza dos seguintes fatos:

\section{FATO 1.1.14}

Seja $\left(k_{1}, k_{2}\right) \in N \times N, k_{1} \neq k_{2}$ e considere $x^{*} \in X^{k_{1} k_{2}}$ e $S=$ $\operatorname{supp}\left\{x^{*}\right\}$. Então para o grafo $G^{*} \equiv\left(N^{*}, E^{*}\right)$ induzido por $S$, isto é, tal que $E^{*}=S$ e $N^{*}=\{i \in N \mid(i, j) \in S$ ou $(j, i) \in S$ para algum $j \in N\}$ tem-se $\forall i \in N^{*} \backslash\left\{k_{1}, k_{2}\right\} \quad \delta(i)>1$

Demonstração

É claro que

$$
\begin{gathered}
M x^{*}=e^{k_{1}}-e^{k_{2}} \Longleftrightarrow \\
\Leftrightarrow \sum_{a \in \mathscr{E}} M(a) x^{*}(a)=\sum_{a \in S} M(a) x^{*}(a)=e^{k_{1}}-e^{k_{3}}
\end{gathered}
$$

e da definição de $M$,

$$
\begin{gathered}
M x^{*}=e^{k_{1}}-e^{k_{2}} \Longleftrightarrow \\
\Leftrightarrow \sum_{(i, j) \in S}\left(e^{i}-e^{j}\right) x^{k}(i, j)=e^{k_{1}}-e^{k_{2}} \Longleftrightarrow \\
\Leftrightarrow \sum_{k \in N^{*}}\left(\sum_{j:(k, j) \in S} x^{*}(i, j)-\sum_{i:(i, k) \in S} x^{*}(i, k)\right) e^{k}=e^{k_{1}}-e^{k_{2}}
\end{gathered}
$$

Assim da independência linear dos vetores $\left\{e^{k}\right\}_{k \in N}$ temos que

$$
\forall k \in N^{*} \backslash\left\{k_{1}, k_{2}\right\} \quad \sum_{j:(k, j) \in S} x^{*}(k, j)=\sum_{i:(i, k) \in S} x^{*}(i, k)
$$

Consequentemente como por construçäo de $G^{*}, \forall k \in N^{*} \mid\left\{k_{1}, k_{2}\right\}, \exists j \in$ $N^{*} \mid x^{*}(k, j)>0$ ou $x^{*}(j, k)>0$, temos que $\forall k \in N^{*} \mid\left\{k_{1}, k_{2}\right\}$, se $\left(k, j^{\prime}\right) \in \operatorname{supp}\left(x^{*}\right)$, então $\exists\left(j^{\prime \prime}, k\right) \in \operatorname{supp}\left(x^{*}\right)$ e portanto $\delta(k)>1$. 
Fato 1.1.15

Nas condições do fato anterior, $\delta\left(k_{1}\right) \geq 1$ e $\delta\left(k_{2}\right) \geq 1$

Demonstração

Usando raciocínio semelhante ao do caso anterior temos

$$
M x^{*}=e^{k_{1}}-e^{k_{2}} \Longleftrightarrow \sum_{k=1}\left(\sum_{j:(j, k) \in E} x^{*}(k, j)-\sum_{i:(i, k) \in E} x^{*}(i, k)\right) e^{k}=e^{k_{1}}-e^{k_{2}}
$$

o que devido a independência linear dos vetores $\left\{e^{k}\right\}_{k}$ implica que

$$
\sum_{j:\left(k_{1}, j\right) \in E} x^{*}\left(k_{1}, j\right)-\sum_{j:\left(j, k_{1}\right) \in E} x^{*}\left(j, k_{1}\right)=1
$$

e

$$
\sum_{j:\left(k_{2}, j\right) \in E} x^{*}\left(k_{2}, j\right)-\sum_{j:\left(j, k_{2}\right) \in E} x^{*}\left(j, k_{2}\right)=-1
$$

donde vem que $\exists j^{\prime} \in N \mid\left(k_{1}, j^{\prime}\right) \in S$ e $\exists j^{\prime \prime} \in N \mid\left(j^{\prime \prime}, k_{2}\right) \in S$ e portanto, pela construção de $G^{*}, \delta\left(k_{1}\right) \geq 1$ e $\delta\left(k_{2}\right) \geq 1$

Esse resultado, juntamente com o anterior, nos mostra que os únicos nós que podem possuir grau $\delta(j) \geq 1$ são os nós $k_{1}$ e $k_{2}$. A partir daítemos

Proposição 1.1.16

Nas condiçóes do fato 1.1.14, temos

Se as colunas de $M / S$ são LI então o grafo induzido $G^{*}$ é conexo.

Demonstração

Como as colunas de $M / S$ são LI, $S$ não contém semi-circuitos pelo teorema 1.1.10, o mesmo valendo para qualquer subconjunto $S^{\prime}$ de $S$. 
Tome uma componente conexa de $G^{*}$. Como o conjunto dos arcos dessa componente não contém semi-circuitos, tornam semi-árvores, a qual possui pelo menos dois nós (cf. apêndice 1) $p_{1} \in N^{*}$ e $p_{2} \in N^{*}$ tais que $\delta\left(p_{1}\right)=1=\delta\left(p_{2}\right)$ que dos fatos 1.1.14 e 1.1.15, implica que $\left\{p_{1}, p_{2}\right\} \subset$ $\left\{k_{1}, k_{2}\right\}$

Logo, toda componente conexa de $G^{*}$ contém os nós $k_{1}$ e $k_{2}$ e portanto $G^{*}$ é conexo.

Esses resultados permitem que provemos:

Proposição 1.1.17

Seja $\left(k_{1}, k_{2}\right) \in N \times N, k_{1} \neq k_{2}$, e $x^{*} \in X^{k_{1} k_{2}}$. Ent ao o grafo $G^{*} \equiv\left(N^{*}, E^{*}\right)$ induzido por $S+\operatorname{supp}\left\{x^{*}\right\}$ tem as seguintes propriedades:

i) $k_{2}$ é atingivel por um passeio de $k_{1}$

ii) Uma das duas afirmaçóes mutuamente exclusivas é válida:

a)ou $G^{*}$ contém semi-circuitos

b)ou $\exists$ ! caminho de $k_{1}$ a $k_{2}$ que utiliza todos os arcos de $G^{*}$

\section{Demonstração}

i)Seja $A$ o conjunto de nós atingíveis a partir de $k_{1} \mathrm{em} S$, isto é, $A=\left\{i \in N \mid \exists \varphi\left(i_{1}, i_{2}, \ldots, i_{r}\right)\right.$ um passeio entre $i_{1}=k_{1} \mathrm{e} i_{r}=i$ tal que $\forall j \in\{1,2, \ldots, r-1\}\left(i_{j}, i_{j+1}\right) \in S$

Fixados dois conjuntos $Z \subset N$ e $Y \subset N(Z \neq \emptyset, N \neq \emptyset)$ chamamos

$$
x^{*}(Z, Y)=\sum_{i \in Z} \sum_{j \in Y:(i, j) \in E} x_{i j}^{*}
$$

Supondo-se $k_{2} \notin A$,e somando-se as equações do problema correspondente aos nós de $\mathrm{A}$ temos

$$
\left.x^{*}(A, N)-x^{*}(N, A)=1 \text { (pois } k_{1} \notin A\right)
$$

Assim, $1=x^{*}(A, N)-x^{*}(N, A)=x^{*}(A, A)+x^{*}(A, \bar{A})-x(\bar{A}, A)-$ $x(A, A)$, onde $\bar{A}=N A$ e portanto $x^{*}(A, \bar{A})-x^{*}(\bar{A}, A)=1$ 
Mas por construção de $A, \forall(i, j) \in(A, \bar{A})$ tem-se $x_{i j}^{*}=0$ e portanto $1=x^{*}(A, \bar{A})-x^{*}(\bar{A}, A)=-x^{*}(\bar{A}, A)$ e como $x^{*} \geq 0,1=-x^{*}(\bar{A}, A) \leq 0$. absurdo

Logo $k_{2} \in A$ e segue a afirmação

ii)Considere $M / S$

a) Se as colunas de $M / S$ são $L D, S$ contém um semi-circuito. b)Se as colunas de $M / S$ são LI, então do fato anterior $G^{*}$ é uma semi-árvore e portanto possue semi-circuitos (teorema 1.1.10). Logo $G^{*}$ é uma semi-árvore e portanto possue pelo menos dois nós $p_{1}$ e $p_{2}$ tais que $\delta\left(p_{1}\right)=\delta\left(p_{2}\right)=1$, isto é, dois nós pendentes. Logo $\left\{p_{1}, p_{2}\right\}=\left\{k_{1}, k_{2}\right\}$ do FATO 1.1.15

Assim, $G^{*}$ é uma semi-árvore com apenas dois nós pendentes 1 e 2 e portanto seus arcos formam um semi-caminho $P=\left(k_{1}, i_{1}, \ldots, i_{r}, k_{2}\right)$ entre 1 e 2 .

Mais ainda,

$$
\begin{aligned}
& M x^{*}= \sum_{a \in E} M(a) x^{*}(a)=\sum_{a \in S} M(a) x^{*}(a)= \\
& \sum_{a \in \mathcal{P}} M(a) x^{*}(a)=e^{k_{1}}-e^{k_{a}}
\end{aligned}
$$

Definamos então $\bar{x} \in \mathbb{Z}^{\mathrm{q}}$,

$$
\bar{x}(a)=\bar{x}(i, j)=\left\{\begin{array}{cl}
+1 & \text { se }(i, j) \in P \\
-1 & \text { se }(j, i) \in P \\
0 & \text { caso contrário }
\end{array} \quad \forall a \in E\right.
$$

(Note que $\bar{x}$ está bem definido pois como $P$ não possui circuitos nä० ocorre $(i, j) \in P$ e $(j, i) \in P)$

É fácil ver que

$$
M \bar{x}=\sum_{a \in E} M(a) \bar{x}(a)=\sum_{a \in P} M(a) \bar{x}(a)=e^{k_{1}}-e^{k_{2}}
$$

Mas por hipótese, as colunas de $M / S$ são LI, donde vem que $\sum_{a \in P} M(a) \bar{x}(a)=\sum_{a \in P} M(a) x^{*}(a) \Longleftrightarrow x^{*}(a)=\bar{x}(a) \forall a \in P$

e claramente $x^{*}=\bar{x}$ e portanto $\bar{x} \geq 0$, donde segue que o semicaminho $P$ é um caminho. 
Seria interessante se pudéssemos caracterizar quais os elementos viáveis em $X^{k_{1} k_{2}}$ que correspondem a caminhos no grafo. Como já vimos, a subconjuntos de colunas da matriz de incidência do grafo que sejam LI podemos associar semi-valores em $G^{*}$. Reciprocamente, a grafos acíclicos, podemos somar subconjuntos LI de colunas da matriz logo é atual tentarmos utilizar propriedades de independência linear ao buscar uma caracterização de caminhos em $G$.

Conforme sabemos, os vértices do conjunto $X^{k_{1} k_{2}}$ estão associados a subconjuntos linearmente independentes de $M$, de tal forma que fica clara a correspondência entre vértices; mais precisamente, adotando a notação e os resultados básicos de [Hermes Hermes] vem

\title{
LEMA 1.1.18
}

$\operatorname{Dados}\left(k_{1}, k_{2}\right) \in N \times N \quad k_{1} \neq k_{2}$ seja $B$ uma base * viável de $X^{k_{1} k_{2}}$

Então o grafo $G^{*}$ induzido pela base $B$,ou seja, formado pelos arcos de $G$ que correspondem às colunas de $M$ associados à base $B$, é uma semiárvore.

Demonstração

Como as colunas de $M$ associadas à base $B$ são LI do TEOREMA 1.1.10 vem que o grafo correspondente não mantém semi-circuitos.

Mas do FATO 1.1.16, esse grafo é conexo e segue a afirmação.

\author{
Além disso temos
}

\section{TEOREMA 1.1.19}

* Utilizamos a notaçào "base" por simplicidade, uma vez que a matriz $M$ não possui característica plena, mas sim, $p-1$. No presente caso, entende-se por base um subconjunto de colunas de $M$ maximal com relação ż independência linear, ou seja, um conjunto de $p-1$ colunas 
Qualquer que seja a base viável $B$ em $X^{k_{1} k_{2}}$ existe um único caminho $P^{*}$ entre $k_{1} \in N$ e $k_{2} \in N$ formado pelos arcos do conjunto $\operatorname{supp}\left\{x^{*}\right\}$ onde $x^{*}$ é o vértice associado a essa base. Para esse caminho $c\left(P^{*}\right)=<$ $c, x^{*}>$.

\section{Demonstração}

Sendo $B$ uma base viável, do lema anterior, o grafo $G^{*}$ induzido é uma semi-àrvore. Como o conjunto dos arcos associados a $B$ contém $S=$ $\operatorname{supp}\left\{x^{*}\right\}$, os arcos de $S$ formam um caminho entre $k_{1}$ e $k_{2}$ (univocamente determinados).

Mas $x^{*}$ é único e, pela prova da PROPOSIÇÃO 1.1.17, dado por

$$
x^{*}(a)= \begin{cases}1 & \text { se } a \in P \\ 0 & \text { caso contrário }\end{cases}
$$

donde segue que $\left\langle c, x^{*}\right\rangle=c\left(P^{*}\right)$

\section{COROLÁRIO 1.1.20}

Qualquer que seja a base viável de $X^{1 p}, \exists$ ! caminho $P$ entre os nós $1 \in N$ e $p \in N$ tal que $c(P)=\left\langle c, x^{*}\right\rangle$ com $x^{*}$ o vértice associado a essa base.

Esse conjunto de resultados poderá nos conduzir, erroneamente, que o problema de determinação de caminho mínimo e o correspondente problema linear fossem equivalentes, o que não ocorre como nos mostra 0 seguinte exemplo.

\section{EXEMPLO 1.1.21}

Seja $G \equiv(N, E) \circ$ grafo

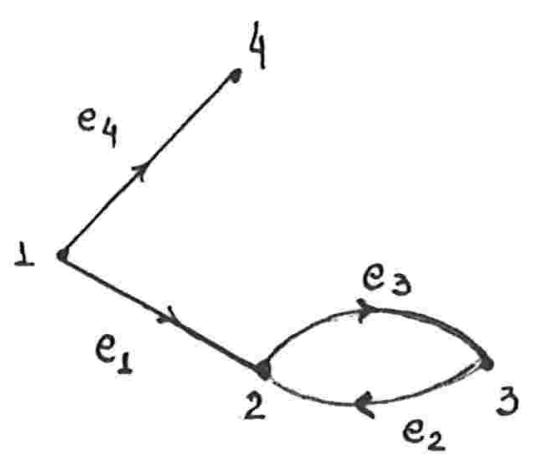

$$
\operatorname{com} N=\{1,2,3,4\}
$$




$$
\begin{gathered}
E=\left\{e_{1}, e_{2}, e_{3}, e_{4}\right\} \text { e } \\
\text { custos } c\left(e_{1}\right)=10 \quad c\left(e_{2}\right)=c\left(e_{3}\right)=-1 \\
c\left(e_{4}\right)=10
\end{gathered}
$$

Claramente o problema de determinação de caminho mínimo entre os nós 1 e 4 possui solução ótima $\phi^{*}=(1,4)$. Entretanto o problema linear correspondente (PMINC) é ilimitado, sendo

$$
y^{*}(\alpha)=\left(\begin{array}{l}
1 \\
0 \\
0 \\
0
\end{array}\right)+\alpha\left(\begin{array}{l}
0 \\
0 \\
1 \\
1
\end{array}\right) \alpha \geqq 0
$$

uma aresta de ilimitacão do problema do caminho mais curto e sua versão em termos de programação linear é válida com uma pequena qualificação como abaixo:

\section{TEOREMA 1.1.22}

Se o problema (PMINC) $\min _{x \in X_{\text {min }}}\left\langle c, x>\right.$ com $x_{\min }=\{x \in$ $\left.\mathbb{R}^{\# \mathbb{E}} \mid \quad M x=e^{1}-e^{p} \quad x \geq 0\right\}$ possui solução ótima $x^{*} e n t \tilde{a} 0 \exists P^{*}$ um caminho mínimo entre os nós $1 \in N$ e $p \in N$ cujo custo $c\left(P^{*}\right)$ é o valor ótimo do problema (PMINC).

\section{Demonstração}

Se (PMINC) possui solução ótima $x^{*}$, então $\exists x^{*} \in X_{\min }$ uma solução ótima básica, $x^{*}=\left(x_{B} \mid 0\right)$ para alguma base ótima $B$. $O$ caminho $P^{*}$ correspondente a $x^{*}$ conforme COROLÁRIO 1.1.20 é portanto um caminho entre os nós 1 e $p$ com custo $c\left(P^{*}\right)=\left\langle c, x^{*}\right\rangle$ cuja otimalidade segue da otimalidade de $x^{*}$. 


\section{COROLÁRIO 1.1.23}

Dada $x^{*}$ solução ótima básica de (PMINC) então o grafo $G^{*} \equiv$ $\left(N^{*}, E^{*}\right)$ induzido por $x^{*}$ é o caminho mínimo $P^{*}$ entre 1 e $p$, sendo que $c\left(P^{*}\right)=\left\langle c, x^{*}\right\rangle$.

Alguns outros exemplos ilustrativos com relação à limitação podem ser construídos:

\section{EXEMPLO 1.1.24}

Considere 0 grafo $G \equiv(N, E)$

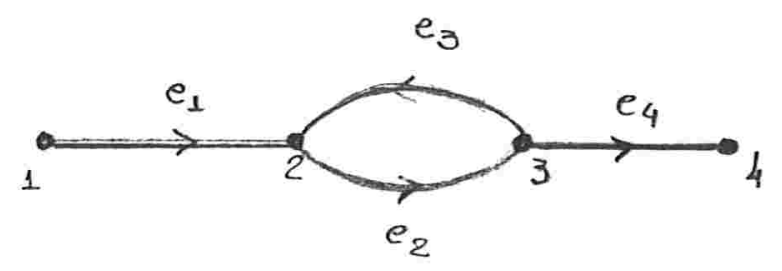

$$
\begin{gathered}
\operatorname{com} N=\{1,2,3,4\} \\
E=\left\{e_{1}, e_{2}, e_{3}, e_{4}\right\} \\
c\left(e_{1}\right)=c\left(e_{4}\right)=10 \\
c\left(e_{3}\right)=-6 \quad c\left(e_{2}\right)=5
\end{gathered}
$$

O problema é claramente ilimitado, sendo que o correspondente problema linear possui como uma aresta de ilimitação

$$
y^{*}(\alpha)=\left(\begin{array}{l}
1 \\
1 \\
0 \\
1
\end{array}\right)+\alpha\left(\begin{array}{l}
0 \\
1 \\
1 \\
0
\end{array}\right)
$$

\section{EXEMPLO 1.1.25}

Seja $G \equiv(N, E)$ 
Capítulo 1: O Problema do caminho mais curto em grafos

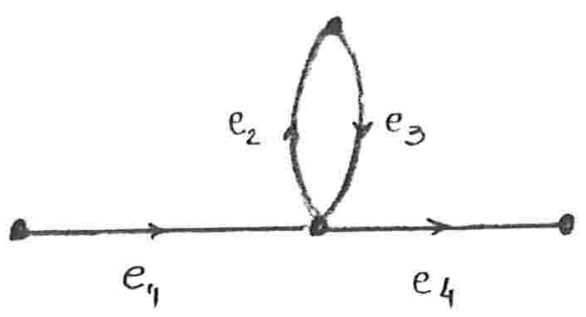

$$
\begin{aligned}
& \operatorname{com} N=\{1,2,3,4\} \\
& E=\left\{e_{1}, e_{2}, e_{3}, e_{4}\right\} \\
& c\left(e_{1}\right)=c\left(e_{4}\right)=1 \\
& c\left(e_{3}\right)=5 \quad c\left(e_{2}\right)=-6
\end{aligned}
$$

Nesse caso, assim como no anterior, tanto o problema de caminho mínimo como o correspondente problema linear são ilimitados

$$
\left(y^{*}(\alpha)=\left(\begin{array}{l}
1 \\
0 \\
0 \\
1
\end{array}\right)+\alpha\left(\begin{array}{l}
0 \\
1 \\
1 \\
0
\end{array}\right)\right)
$$

Nos três exemplos de ilimitação vistos, o que permitiu a construção de arestas de ilimitação foi a existência de circuitos de custo negativo no grafo.

Lembremos que a aresta de ilimitação de problemas lineares estão associados elementos de um cone, no nosso caso especificamente $H=\{h \in$ $\left.\mathbb{R}^{\mathrm{q}} \mid M \cdot h=0 \quad h \geqq 0\right\}$. Mais precisamente o problema (PMINC) é ilimitado se e somente se $\exists \bar{h} \in H$ tal que $\langle c, \bar{h}\rangle\langle 0$ ([Humes Humes]).

Observe que aos elementos do conjunto $H$, podemos associar, de forma natural, circulaçóes positivas definidas sobre os arcos do grafo (assim como fizemos no LEMA 1.1.10) e isso sugere que relacionemos entăo esse cone com circuitos no grafo.

\section{LEMA 1.2.26}

Seja $H=\left\{h \in \mathbb{R}^{\# E} \mid M \cdot h=0 \quad h \geqq 0\right\}$ se $H \neq\{0\}$ então o grafo $G \equiv(N, E)$ contém semi-circuitos.

\section{Demonstração}


Identificando os elementos de $H$ com funções definidas sobre as arestas de $G$, temos que $H=\left\{h: E \rightarrow \mathbb{R} \mid \sum_{\mathrm{a} \in \mathrm{E}} \mathrm{M}(\mathrm{a}) \mathrm{h}(\mathrm{a})=0 \mathrm{~h}(\mathrm{a}) \geqq 0\right.$ $\forall a \in E$ \} e portanto $H \subset W_{C}$ (o espaço vetorial das circulações).

Como $H \neq\{0\}, \exists \hat{h}(\cdot) \in H$ uma circulação não nula em $G$ e portanto $\operatorname{supp}(\hat{h}) \subset E$ contém um semi-circuito (do LEMA 1.1.9).

A recíproca desse lema não é verdadeira pois a semi-circuitos no grafo podemos, segundo o TEOREMA 1.1.10, associar colunas de $M$ linearmente dependentes de forma que $M \cdot h=0$ para algum $h \neq 0$, a qual não satisfaz necessariamente e $h \geqq 0$. Para que possamos garantir a não negatividade de $H$ devemos supor condições mais fortes

\section{PROPOSIÇÃO 1.1.27}

A cada circuito $C^{*}$ em $G \equiv(N, E)$, existe associado $h^{*} \in H=\{h$
$\left.\in \mathbb{R}^{\# E} \mid M h=0 \quad h \geq 0\right\}$ tal que $\left\langle c, h^{*}>=c\left(C^{*}\right)\right.$.

Demonstração

Na mesma linha que na demonstração do TEOREMA 1.1.10, considere o vetor $y \in \mathbb{R}^{\# \mathbb{E}}$, definido por

$$
y(i, j)= \begin{cases}1 & \text { se }(i, j) \in C \\ 0 & \text { caso contrário }\end{cases}
$$

que claramente satisfaz $M y=0$

Sendo $y \geqq 0, y \in H$

Por construção segue que $\langle\epsilon, y\rangle=c\left(C^{*}\right)$

Para esse resultado vale a recíproca

\section{PROPOSIÇÃO 1.1.28}

Se $H=\left\{h \in \mathbb{R}^{\# \mathbb{E}} \mid M \cdot h=0 \quad h \geqq 0\right\}$ 
Se $H \neq\{0\}$ então o grafo $G \equiv(N, E)$ contém circuitos.

\section{Demonstração}

Identificando $H$ com o espaço das funções, isto é, $H=\{h: E \rightarrow$ $\mathbb{R} \mid \sum_{\mathrm{a} \in \mathrm{E}} \mathrm{M}(\mathrm{a}) \mathrm{h}(\mathrm{a})=0 \mathrm{~h}(\mathrm{a}) \geq 0$ foralla $\left.\in \mathrm{E}\right\}$

Qualquer que seja $h(\bar{\cdot}) \in H \backslash\{0\}$, o grafo $\bar{G}=(\bar{N}, \bar{E})$, induzido por $\bar{E}=\operatorname{supp}\{\bar{h}\}$ (conforme FATO 1.1.14) contém semi-circuitos.

Tomemos $h^{*}(\cdot) \in H$, minimal com relação a esta propriedade, isto é, $I^{*}=\operatorname{supp}\left\{h^{*}\right\}=\left\{a_{1}, a_{2}, \ldots, a_{r}\right\}$ contém um semi-circuito $\forall j \in I^{*}, I^{*} \backslash$ $\{j\}$ não contém semi-circuitos.

Tome $a_{j} \in I^{*}$ qualquer, $a_{j}=\left(j_{1}, j_{2}\right)$, e seja $J^{*}=I^{*} \backslash\left\{a_{j}\right\}$ claramente $\sum_{a \in E} M(a) h^{*}(a)=\sum_{a \in I^{*}} M(a) h^{*}(a)=\sum_{a \in I^{*}} M(a) h^{*}(a)+$ $M\left(a_{j}\right) h^{*}\left(a_{j}\right)=0$

ou equivalentemente $\sum_{a \in I^{*}} M(a) \frac{h^{*}(a)}{h^{*}\left(a_{j}\right)}=e^{j_{1}}-e^{j_{2}}$.

Chamando $h(a)=\frac{h^{*}(a)}{h^{*}\left(a_{j}\right)}$, estamos nas condiçóes da PROPOSIÇÃO 1.1.17 e existe um caminho entre $j_{1}$ e $j_{2}$ que utiliza todos os arcos de $J^{*}$. Sendo a escolha de $a_{j}$ arbitrária, vem que $I^{*}$ contém um circuito.

A inexistência de circuitos de custos negativos em grafos finitos é uma condição suficiente, para que o problema de determinação do caminho mínimo, se viável, possua solução ótima. É usual ao estudarmos problemas de busca em grafos, admitiremos tal hipótese e segundo o resultado anterior, ao fazê-lo estamos assumindo implicitamente que o correspondente problema linear não é ilimitado.

Sob essa hipótese, ao utilizarmos por exemplo, o método simplex para a resolução do problema (PMINC), das soluçōes ótimas obtidas podemos extrair caminhos mínimos no grafo. Apesar disso, um algoritmo geral tipo simplex não se aproveita da estrutura de grafos do problema, o que justifica a tentativa de utilização de algoritmos que a explore. Conduziremos a apresentação deste tipo de algoritmo em conjunto com o estudo de dualidade para (PMINC), o que nos permitirá relacionar algoritmos em grafos com dualidade.

\subsection{O DUAL DO PROBLEMA DO CAMINHO MAIS CURTO}


Assumiremos aqui que é conhecido o processo de construção de duais de problemas lineares segundo a abordagem de Lagrange, bem como os principais resultados sobre dualidade tais como teoremas forte e fraco e conceitos como folgas complementares, conforme apresentado a nível de graduação em [Humes \& Humes].

Adotando-se a formulaçã̃o do problema em termos de programação linear (PMINC), estudemos seu dual.

\section{FATO 1.2.1}

$O$ dual de (PMINC) é dado por

(DMINC): "Encontre, se existir, $u^{*} \in \mathbb{R}^{\# N}$ tal que $u^{*} \in$ arg $\max _{u \in U_{D M I N C}}\left\{u_{1}-u_{p}\right\}$, onde $U_{D M I N C}=\left\{u \in \mathbb{R}^{\# N} \mid u_{\mathrm{i}}, \mathrm{u}_{\mathrm{j}} \leqq \mathrm{c}_{\mathrm{ij}} \forall(\mathrm{i}, \mathrm{j}) \in\right.$ E\}".

Demonstração

Segue que nos moldes clássicos, lembrando que a matriz de coeficientes do problema é da forma $M(a)=e^{i}-e^{j} \forall a=(i, j) \in E$

Um resultado que nos será util posteriormente, relaciona as soluções otimas dos dois problemas

\section{LEMA 1.2.2 (FOLGAS COMPLEMENTARES) \\ Sejam $x \in \mathbb{R}^{\# \mathbf{E}}, u \in \mathbb{R}^{\# \mathbf{N}}$}

$\left.\begin{array}{l}\mathrm{x} \text { é solução ótima de (PMINC) } \\ \mathrm{x} \text { é solução ótima de (DMINC) }\end{array}\right\} \Leftrightarrow\left\{\begin{array}{l}u \in U_{\text {DMINC }} \\ x \in X_{\min } \\ x_{i j}\left(c_{i j}-u_{i}+u_{j}\right) \\ \forall(i, j) \in E\end{array}\right.$

Demonstração 

blema dual (DMINC)

O método por ser um resultado clássico particularizado para o pro-

Como estamos trabalhando com problemas lineares, vale o teorema forte de dualidade, que nos assegura que os valores ótimos primal e dual sấo iguais. No nosso caso, em particular, é possível a partir de uma solução ótima primal, uma solução ótima dual. Antes porém de verificarmos esse fato fixemos uma definição que será adotada até o final do trabalho.

\section{DEFINIÇÃO 1.2 .3}

Dado um grafo orientado $G \equiv(N, E)$ com custos associados às arestas, definimos por $h(\cdot, \cdot)$ à função distância do grafo, isto é,

$$
\begin{gathered}
k: N \times N \rightarrow \overline{\mathbb{R}} \\
k(i, j)=\inf \{c(P) \mid P \text { eum caminho entre i e j }\}
\end{gathered}
$$

Em particular, para uma fixada origem $1 \in N$, denotamos por $g(\cdot)$, à função

$$
\begin{gathered}
g: N \rightarrow \overline{\mathbb{R}} \\
g(i)=k(1, i)
\end{gathered}
$$

Nessas condições vamos construir variáveis dual viáveis a partir de caminhos mínimos. (DMINC)

\section{TEOREMA 1.2.4 (CONSTRUÇÃO DE VARIÁVEIS DUAIS)}

Considere o problema de caminho mínimo (PMINC) e seu dual

Se (PMINC) possue solução ótima e $\forall i \in N g(i) \in \mathbb{R}$ então $u^{*} \in$ $\mathbb{R}^{\# N}$ definida por $u_{i}^{*}=-g(i) \forall i \in N$ é uma solução ótima dual

Demonstração 
Seja $(i, j) \in E$ Como $g(j) \leqq g(i)+c_{i j}$, temos $u_{i}^{*}-u_{j}^{*}=-g(i)+$ $g(j) \leqq c_{i j}$ e portanto $u^{*}$ é viável dual.

Por outro lado, como (PMINC) possue solução ótima $u^{*}$, o grafo $G \equiv(N, E)$ não possue circuitos de custo negativo (da PROPOSIÇÃO 1.1.25) portanto $g(1)=0$. Além disso, $\left\langle c, x^{*}\right\rangle=g(p)$. Assim, $u_{1}^{*}$ viável segue a tese.
.

Assim, $u_{1}^{*}-u_{p}^{*}=-g(1)+g(p)=g(p)=<c, x^{*}>$ e como $u^{*}$ é

A hipótese de $\forall i \in N g(i) \in \mathbb{R}$ pode ser vista como uma forma de indicar que todos os nós são atingíveis a partir da origem, o que ocorre nos casos em que estaremos particularmente interessado (mais precisamente, na resolução de problemas de grupo).

As condiçōes de folgas complementares estão associadas a condições necessárias e suficiente de otimalidade que nos permitem garantir a validade algoritmos (primais ou duais) e portanto é de se esperar que algoritmos que explorem a estrutura de grafos e dualidade (implícita ou explicitamente) utilizem essa condição. Assim, uma idéia para a obtenção de algoritmos para detecção de caminhos mínimos consiste em construir uma sequiência de elementos dual viáveis mantendo folgas complementares e de tal forma a se obter a solução ótima $u^{*}$ construída no teorema anterior numa linha análoga à do simplex dual.

Cabe observar uma peculiaridade deste problema dual.

Se considerarmos para una fixada solução dual viável $u$, a função $p_{u}(\cdot)$ definida por $p_{u}: N \rightarrow \mathbb{R} p_{u}(\mathrm{i})=u_{\mathrm{i}}$ e tomarmos $f_{u}: E \rightarrow \mathbb{R} f_{u}(\mathrm{i}, \mathrm{j})=$ $\mathrm{p}_{\mathrm{u}}(\mathrm{i})-\mathrm{p}_{\mathrm{u}}(\mathrm{j})$ uma função diferença de potencial, o problema dual(DMINC) pode ser reescrito como

$$
\max f_{u}(1, p) \quad f_{u}(i, j) \leqq c_{i j} \forall(i, j) \in E \forall u \in \mathbb{R}^{\# N}
$$

ou seja, (PMINC) pode ser visto como o problema de determinação da função diferença de potencial que maximiza $f_{u}(1, p)$ para um fixado conjunto de funções diferença de potencial e obedece à restrição $f_{u}(i, j) \leqq c_{i j}$

Observe que nesse caso, $f_{z}(i, j)=\left\langle\omega, e^{i}-e^{j}\right\rangle \forall(i, j) \in E$, ou seja, estamos considerando funçổes $f_{u}(\cdot, \cdot)$ "lineares", definidas abre os arcos de $G$. 
Se reescrevermos o problema dual (DMINC) na forma

$$
\begin{aligned}
& (\mathrm{DMINC}) \max <u, e^{1}-e^{p}> \\
& \left\langle M(i, j), u>\leqq c_{i j} \forall(i, j) \in E \quad \forall u \in \mathbb{R}\right.
\end{aligned}
$$

(com $M$ a matriz de coeficientes do problema), e considerarmos que as funçöes lineares definidas sobre $\mathbb{R}^{\# \mathbb{N}}$ se escrevesse na forma $\varphi(\cdot)=\left\langle e^{*}, \cdot\right\rangle$ para algum $e^{*} \in \mathbb{R}^{\# N}$, o problema dual pode ser interpretado como a determinação da função linear $\varphi(\cdot)$ definida em $\mathbb{R}^{\# N}$ que maximiza o valor $\varphi\left(e^{1}-e^{p}\right)$.

Assim o problema dual (DMINC) é equivalente a

$$
\max \varphi\left(e^{1}-e^{p}\right) \varphi(M(i, j)) \leqq c_{i j} \quad \forall(i, j) \in E \quad \forall \varphi(\cdot) \in \mathcal{L}
$$

$\mathcal{L}$ o espaço vetorial das funções lineares definidas em $\mathbb{R}^{\# N}$.

Esse tipo de enfoque nos será bastante util quando passarmos ao estudo da relação entre algoritmos enformados para a resolução de problemas de busca em grafos e dualidade em um sentido mais amplo.

\section{3 - CONCLUSÃO}

Do que foi exposto, podemos extrair um enfoque para resolução do problema do caminho mínimo, através da utilização de métodos que explorem a estrutura de grafos do problema, construindo iterativamente, elementos viáveis no dual do correspondente problema linear.

É natural que algoritmos que busquem caminhos mínimos pesquisem os nós do grafo iterativamente, buscando determinar o valor $g(i)$ para cada $i \in N$. Nossos dois próximos capitulos estudam alguns desses algoritmos, um deles "ótimo" no sentido de "pesquisar" o menor número possível de nós. No capítulo que segue estudamos os dois algoritmos mais clássicos, quais sejam os algoritmos de Dijkstra e o de Ford. 


\section{Capítulo 2}

\section{Algoritmos de Dijkstra e Ford}

Dentre os algoritmos aplicáveis a resolução de problemas de busca de caminho mínimo em grafos, encontramos uma particular classe, que chamaremos de algoritmos de rotulação, que pesquisam iterativamente os nós do grafo, associando aos mesmos um conjunto de informaçóes quanto a algum caminho entre a origem e o nó sendo pesquisados.

Alguns dos algoritmos de rotulação mais conhecidos são o algoritmo de Dijkstra, aplicável a grafos cuja função custo seja nảo negativa, algoritmos de busca em profundidade e busca horizontal, o de Ford e finalmente o algoritmo $A^{*}$, inicialmente estudado na área de inteligência artificial.

A bibliografia no que se refere à resolução do problema é extensa e nosso estudo será basicamente restrito ao estudo dos algoritmos de Dijks- 
tra, Ford e $A^{*}$ devido a relação que os mesmos mantém com os duais do problema do caminho mais curto.

\subsection{O ALGORITMO DE DIJKSTRA E DUALIDADE}

A descrição do algoritmo de Dijkstra tem como referência básica - trabalho de Dijkstra [Di59], sendo que trabalhos similares, como os de Moore [Mo59], o de Whiting and Hillier [WH60] e o de Dantrig [Da59] entre outros podem ser encontrados na literatura. Outras referências historicamente interessantes podem ser encontradas no trabalho de Dreyfus [Dr68].

O princípio básico do processo consiste em associar rótulos aos nós do grafo $(j \in N)$ que correspondam a um limite superior do custo do caminho mínimo entre a origem e o nó $j$. Os rótulos dos nós sáo subdivididos em dois tipos: temporários e permanentes, sendo que os segundos correspondem ao custo de um caminho mínimo entre a origem e o nó rotulado. Mais ainda, em cada iteração do algoritmo faz-se uma partição dos nós em nós sem rótulo, com rótulo permanente e nós com rótulos temporários, sendo que ao conjunto de nós com rótulo permanente denominamos fechados enquanto que aos com rótulo temporário: abertos.

A idéia fundamental do processo é a deteç̧ão do custo do caminho mínimo entre a origem e algum elemento aberto, com fechamento do mesmo e a subsequente atualização do conjunto de nós adjacentes a ele, quer por inclusão de nós que não foram nem abertos nem fechados no conjunto de abertos, ou por atualizaçao de rótulos dos abertos.

Dois aspectos caracterizam o algoritmo em termos operacionais:

i) é aplicavél apenas a grafos com custos não negativos, 0 que apesar de restritivo cobre uma classe ampla de problemas.

ii) é eficiente em termos computacionais, tanto em termos imprecisos quanto no sentido formal de complexidade.

Para indicar a restrição imposta pelo método em relação a custos nảo negativos, nos referiremos à seguinte hipótese

\section{HIPÓTESE 2.1.1}


$\mathbb{R}^{+}$

A função custo associada ao grafo é não negativa, isto é, $c: N \rightarrow$

Utilizaremos também as hipóteses assumidas no capítulo 1 quanto à finitude, conexidade e inexitência de laços. Mais precisamente iremos associar a HIPÓTESE BÁSICA e a HIPÓTESE 1.1.2.

Ao conjunto de nós com rótulos permanentes (nós fechados) na iteração $k$ chamaremos $C^{k}$, e aos com rótulos temporários (abertos) de $O^{k}$. Tendo admitido que o conjunto de nós do grafo é finito, $N=\{1,2, \ldots, p\}$ vamos denominar o rótulo de um dado nó $j \in N$ na iteração $k \in \mathbb{N}$ por $f^{(k)}(j)$. Descrevendo então o algoritmo teremos:

\section{ALGORITMO 2.1.2 O ALGORITMO DE DIJKSTRA}

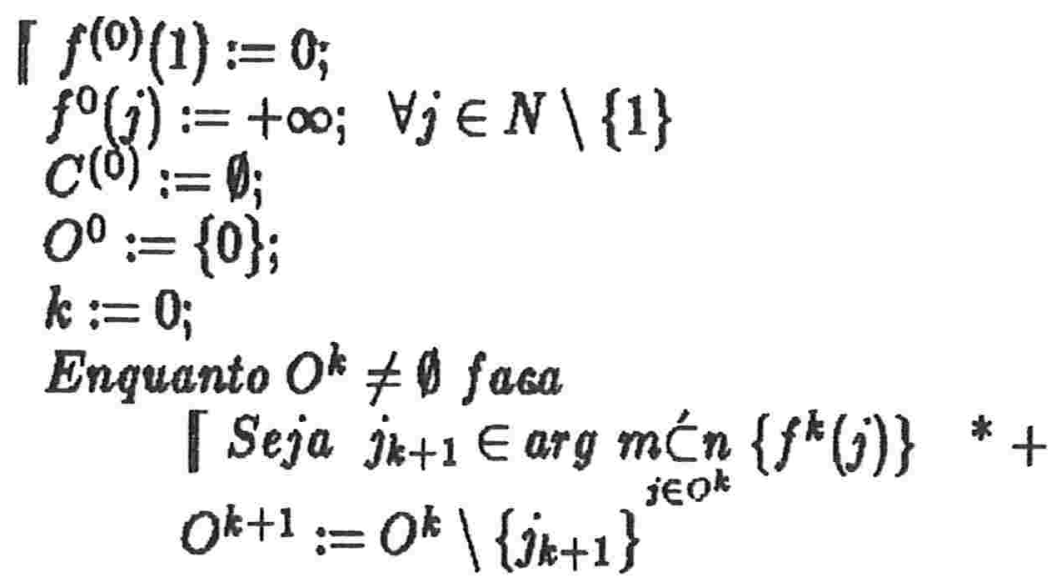

* Para que fossemos mais precisos deveríamos tomar a função in $\{\{\}$ ao invés de $\min \{\}$ na expressäo, pois eventualmente pode ocorrer $f^{k}(j)=$ $+\infty \forall j \in O^{k}$; ainda assim adotaremos o "mínimo" para que a notação seja consistente com o trabalho posterior. Poderíamos assumir sem perda de generalidade, que inicialmente $f^{k}(j)=M \forall j \in O^{k} \backslash\{1\}$, onde $M$ é um número suficientemente grande (por exemplo, $M \geqq[(\# E)+1] * \max _{o \in E}\{c(e)\}$ e com isso não haveria problemas em adotarmos tal nomenclatura.

$+\mathrm{Na}$ forma proposta o método não se caracteriza como um algoritmo uma ver que nos casos de "empate", isto é, quando arg $\min _{j \in O^{k}}\left\{f^{k}(j)\right\}>1$, não determinamos um critério de escolha de elemento para fechamento. Apesar disso não haverá prejuízo ao adotarmos tal nomenclatura, usual na literatura. 


$$
\begin{aligned}
& C^{k+1}:=C^{k} \cup\left\{j_{k+1}\right\} \\
& \forall j \in N \text { faga } \\
& \text { se } j \notin C^{k+1} \text { e } j \in \operatorname{suc}\left(j_{k+1}\right) \\
& \text { então } \\
& k:=k+1 ;
\end{aligned}
$$

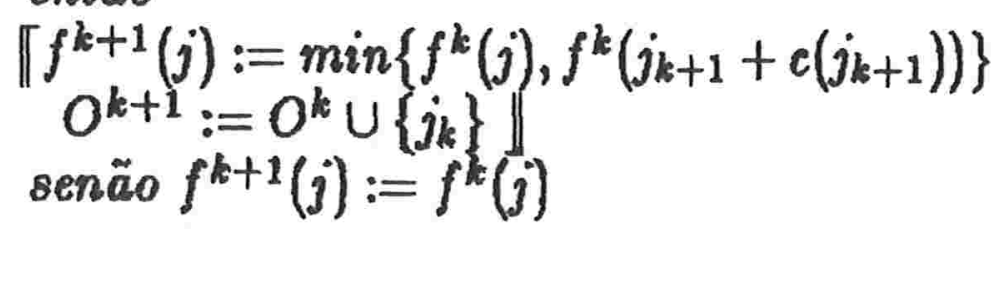

\section{FIM - ALGORITMO}

Uma observação cabe ser feita neste ponto : 0 algoritmo de Dijkstra , conforme descrito usualmente, possui como critério de parada a condição $p \in C^{k}$ ao invés de $O^{k} \neq \emptyset$, porém iremos adotar tal critério para que haja consistência com o restante do trabalho.

Em casos triviais como no seguinte grafo

$$
G \equiv(N, E) \quad N=\{(2,1)\} \quad E=\{(2,1)\}
$$

- nó alvo (no caso, 2 e $N$ ) só é incluído no conjunto de elementos fechados com valor $f^{k}(p)=+\infty\left(f^{k}(2)=+\infty\right)$, o que nos leva a indagar se não seria esta uma condição suficiente para deteç̧ão de inviabilidade do problema. Isso é verdade e, ainda mais geralmente, caso $f^{k}(p) \in \mathbb{R}$, um caminho mínimo pode ser obtido entre a origem e 0 alvo.

Um primeiro aspecto a ser observado é que ao associarmos um rótulo $f^{k}(j) \in \mathbb{R}$ a um nó, teremos atingido, pelo processo de construção do algoritmo, esse nó $j \in N$ a partir da origem por um passeio de custo $f^{k}(j)$. Portanto, do processo de fechamento de nós, ao escolhermos $j_{k} \in N$ cujo valor $f^{k}\left(j_{k}\right)$ seja mínimo, o correspondente passeio entre 1 e $j$ deve possuir custo mínimo, de tal forma que os nós fechados possuam rótulos $f^{k}(j)=g(j)$ (onde $g(j)$, conforme DEFINIÇÃO 1.2.3 é o custo de um caminho mínimo entre 1 e $j$ ).

A verificação desses resultados se processa como segue 


\section{PROPOSIÇÃO 2.1.3}

Seja $k \in \mathbb{N}$ uma iteração qualquer do algoritmo de Dijkstra.

Então $\forall j \in C^{k} \cup O^{k}$ existe um passeio $P$ entre $i$ e $j$ de custo $c(p)=f^{k}(j)$

\section{Demonstração}

Por indução sobre o número de iterações do algoritmo.

Base da indução $(k=1)$

Nessa iteração, $C^{1}=\left\{1, j_{1}\right\}$ e $O^{1}=\operatorname{suc}(1) \backslash\left\{j_{1}\right\}$

Seja então $j \in C^{1} \cup O^{1}$

Se $j=1 f^{1}(j)=f^{0}(j)=0$ e nada a fazer

Se $i \in \operatorname{suc}(1)$ então $f^{1}(j)=j^{0}(1)+c(1, j)=c(1, j)$ e a afirmação segue tomando-se $p_{j}=(1, j)$

Hipótese de indução

Suponha que $\forall k 1 \leqq k \leqq p-1$ a afirmação seja verdadeira, isto é,

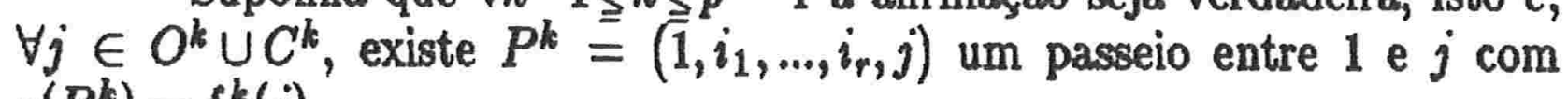
$c\left(P^{k}\right)=f^{k}(j)$

$k=p$

Seja $j \in O^{k} \cup C^{k}$

Se $f^{k}(j)=f^{k-1}(j)$ a afirmação segue da hipótese de indução.

Se $f^{k}(j) \neq f^{k-1}(j)$ então esse nó teve seu rótulo alterado nesta iteração e $f^{k}(j)=f^{k-1}\left(j_{k}\right)+c\left(j_{k}, j\right), j_{k}$ o nó escolhido para fechamento nessa iteração.

Mas da hipótese de indução, como $j_{k} \in O^{k-1}$, temos que $\exists P_{j_{k}}^{k-1}=$ $\left(1, i_{1}, \ldots, i_{r}, j_{k}\right)$ com $c\left(P_{j_{k}}^{k-1}\right)=f^{k-1}\left(j_{k}\right)$ e tomando-se o passeio $P_{j}^{k}=$ $\left(1, i_{1}, \ldots, i_{r}, j_{k}, j\right)$ segue a afirmação.

Mostramos assim que aos nós fechados ou abertos pelo algoritmo estäo associados passeios cujo custo é dado pelos seus rótulos.

Segue trivialmente deste resultado que 


\section{LEMA 2.1.4}

Sendo $k \in \mathbb{N}$ uma iteração do algoritmo de Dijkstra tem-se $\forall j \in$ $N \quad f^{k}(j) \geqq g(j)$

Demonstração

Seja $j \in N$

anterior

Se $j \in O^{k} \cup C^{k}$, a afirmação segue trivialmente da proposição

Se $j \notin O^{k k}$ então claramente $f^{k}(j)=+\infty$ e portanto $f^{k}(j) \geqq g(j)$

Como consequência vem que

\section{COROLÁRIO 2.1.5}

Seja $j \in N$

origem.

Se $\exists k \in \mathbb{N}$ tal que $f^{(k)}(j) \in \mathbb{R}$, então $j$ é atingível a partir da

Observe que esses resultados são válidos para uma classe bastante ampla de algoritmos que rotulam os nós do grafo considerado. $\mathrm{O}$ que distingue Djkstra dos demais algoritmos é a utilização de um critério de pesquisa de nós que permite que ao fechar um nó tenhamos determinado 0 custo de um caminho mínimo da origem até ele.

\section{TEOREMA 2.1.6}

Seja $k \in \mathbb{N}$ uma iteração qualquer do algoritmo de Dijkstra.

Então $\forall j \in C^{k}, f^{k}(j)=g(j)$

Demonstração

Por indução sobre o número de iteração :

$k=0$ nada a farer 
$k=1$

Seja $\left.j \in C^{1}=\{1, j)\right\}$

Se $j=1 f^{1}(1)=f^{0}(1)=0=g(1)$

Se $j \neq 1$ então do critério de escolha para fechamento de nós, $f^{1}(1) \leqq f^{1}(i) \forall i \in O^{1}=\operatorname{suc}(1)$ e portanto $c(1, j) \leqq c(1, i) \forall i \in O^{1}$

Mas por hipótese os custos do grafo são não negativos e portanto qualquer passeio entre 1 e $j$ näo terá custo inferior a $P(1, j)$. Assim $f^{1}=$ $c(1, j)=g(j)$.

Hipótese de indução

Suponha que $\forall k \quad 1 \leqq k \leqq p-1, \forall j \in C^{k} \quad f^{k}(j)=g(j)$

$k=p$

Seja $j \in C^{k}$

Se $j \in C^{k-1}$, da hipótese de indução $f^{k-1}(j)=g(j)$ e como rótulos de elementos fechados não são alteradas, $f^{k}(j)=f^{k-1}(j)=g(j)$. iteração.

Se jin $C^{k} \backslash C^{k-1}$ então $j$ foi o nó escolhido para fechamento nesta

Suponhamos que $f^{k}(j)>g(j)$ (sabemos que $\left.f^{k}(j) \geq g(j)\right)$ e considere um caminho mínimo entre 1 e $j, P=\left(i_{1}, i_{2}, \ldots, i_{r}\right)$. Seja $g^{*} \in$ $\{1,2, \ldots, r\}$ o maior índice para o qual $\forall l 1 \leqq l \leqq s^{*} i_{l} \in C^{k-1} i_{s^{*}+1} \notin C^{k-1}$ $\left(s^{*}\right.$ existe pois $1 \in C^{k-1}$ e $\left.j \notin C^{k-1}\right)$. Da hipótese de indução $f^{k}\left(i_{s^{*}}\right)=$ $f^{k-1}\left(i_{z^{*}}\right)=g\left(i_{z^{*}}\right)$ e portanto $f^{k}\left(i_{s^{*}}+1\right) \leqq g\left(i_{z^{*}}+1\right)+c\left(i_{z^{*}}, i_{g^{*}}+1\right)=g\left(i_{z^{*}+1}\right)$ Mas $f^{k}\left(i_{s^{*}}+1\right) \geqq g\left(i_{s^{*}}+1\right)$ e temos então $f^{k}\left(i_{s^{*}+1}\right)=g\left(i_{s^{*}+1}\right)$

Como $i_{s^{*}+1} \notin C^{k-1}$ e $i_{s^{*}} \in C^{k-1}$, temos que $i_{s^{*}+1} \in O^{k-1}$ e além disso dendo os custos do grafo não negativos, $f^{k}\left(i_{s^{*}+1}\right)=g\left(i_{s^{*}+1}\right) \leqq g(j)<$ $f^{k}(j)$ o que contraria a escolha de $J$ para fechamento.

Assim $f^{k}(j)=g(j)$

Do princípio de indução segue a afirmação.

Esse resultado nos assegura que se o problema for viável e o nó $p$ for fechado pelo algoritmo, teremos determinado o valor ótimo do problema, isto é, obtemos o custo de um caminho mínimo entre a origem e 0 alvo. 
O critério de rotulação do algoritmo possibilitou que associássemos passeios a cada nó aberto ou fechado pelo algoritmo. Como pudemos garantir que para elementos fechados, $f^{k}(j)=g(j)$, sendo $P_{j}$ o passeio entre a origem e o nó $j$ de custo $c\left(P_{j}\right)=f^{k}(j)=g(j)$, caso o grafo não possua circuitos de custo nulo, $P_{j}$ é um caminho.

Podemos garantir também que o algoritmo termina em um número finito de passos, quando então terá determinado o custo de um caminho mínimo entre a origem e cada nó atingível a partir dela (em particular para o nó p caso o mesmo seja atingível).

\section{TEOREMA 2.1.7}

Seja $G \equiv(N, E)$ um grafo orientado satisfazendo as HIPÓTESES 1.1.2, 2.1.1 e HIPÓTESE BÁSICA.

Então o algoritmo de Dijkstra aplicado a $G$ termina em um número finito $k^{*}\left(k^{*} \leqq \# N\right)$ de passos, quando então $O^{k^{*}}=\emptyset \mathrm{e} \forall j \in C^{k^{*}} f^{k^{*}}(j)=$ $g(j)$.

\section{Demonstração}

Da finitude do grafo, como a cada iteração um nó é escolhido para fechamento não sendo mais reaberto, segue que após um número finito $k^{*}\left(k^{*} \leqq \# N\right)$ de passos $O^{k^{*}}=\emptyset \mathrm{e} 0$ algoritmo termina. $g(j)$

Nesse ponto, do resultado anterior segue que $\forall j \in C^{k^{*}} f^{k^{*}}(j)=$

O algoritmo de Dijkstra pode ser considerado um caso particular de outro algoritmo conhecido como $A^{*}$, que estudaremos em detalhe adiante, sendo que podemos assim assegurar a validade de Dijkstra como uma consequência imediata da finitude e validade de $A^{*}$. Não optamos por essa linha pois não ficariam claras algumas peculiaridades de Dijkstra que inclusive simplificam as demonstraçóes utilizadas ( por exemplo, o fechamento 
apenas de nós cujo rótulo corresponda ao custo de um caminho mínimo). Além disso, a notação lá utilizada dificulta a compreensão daqueles não familiarizados com o método.

Outro resultado válido para Dijkstra é o seguinte :

\section{PROPOSIÇÃO 2.1.8}

Nas condiçōes do teorema anterior temos que após um número finito $k^{*}$ de passos $\forall j \in N, j$ é atingível a partir de 1 tem-se $j \in C^{k^{*}}$

\section{Demonstração}

Sendo $j \in N$ atingivel a partir de 1 sabemos que existe um caminho mínimo entre 1 e $j$ (pois o grafo por hipótese é finito com custos não nega. tivos). Considere então $P^{*}=\left(i_{1}, i_{3}, \ldots, i_{r}\right)$ e seja $k$ uma iteração qualquer do algoritmo e seja $s^{*}, 1 \leqq s^{*} \leqq r$ o maior índice para o qual $\forall l, 1 \leqq l \leqq s^{*} i_{l} \in C^{k}$. Se $s^{*}=r$ nada a fazer, $j \in C^{k}$ Se $s^{*}<r$ então o nó $i_{s^{*}+1} \in O^{k}$ e portanto $O^{k} \neq \emptyset$ de forma que o algoritmo não termina nessa iteraçäo. Como ao final de um número finito $k^{*}$ de passos $O^{k^{*}}=1$ segue a afirmação.

A partir daí temos a seguinte

\section{PROPOSIÇÃO 2.1.9}

Nas condições do TEOREMA 2.1.7 na iteração $k^{*}$ em que 0 algoritmo termina, tem-se que:

Se $j \in N$ é atingivel a partir de 1 , então qualquer caminho mínimo entre 1 e $j$ possue todos os nós em $C^{k^{*}}$.

\section{Demonstração}

Do resultado anterior temos que na iteração em que 0 algoritmo termina, todos os nós atingfveis a partir da origem estaräo fechados. Em particular, sendo $P=\left(i_{1}, i_{2}, \ldots, i_{p}\right)$ um caminho mínimo entre $i_{1}=1 \mathrm{e}$ $j=i_{r}$ vale a afirmação e portanto $\forall l \quad 1 \leqq l \leqq r \quad i_{l} \in C^{h^{*}}$ 
Assim, ao final do algoritmo temos que os caminhos mínimos entre a origem e os nós podem ser recuperados através de pesquisa no conjunto $C^{k^{*}}$. A fim de facilitar tal determinação de caminhos podemos associar aos nós $j \in N$ alguma informação quanto ao elemento que o antecede no caminho $P$ entre 1 e $j$ associado a ele em uma dada iteração $k$, isto é, sendo $P=\left(1, i_{1}, i_{2}, \ldots, i_{r}, j\right)$ podemos definir o antecessor de $j$ como $a^{k}(j)=i_{r}$. Com isso, ao final do algoritmo basta aplicar sucessivamente esse operador e teremos determinado o caminho.

A verificação de que isso é possível será feita ao estudarmos $A^{*}$ sendo por hora omitida.

Observe que poderíamos utilizar o critério de parada usualmente utilizado por Dijkstra, isto é, $p \in C^{k}$ pois ao final de um número finito de passos o nó $p$, se atingível a partir de 1 , é inclú́do no conjunto $C^{k}$ com $f^{k}(p)=g(p)$.

O processo de pesquisa de nós poderia ser "acelerado" no sentido de diminuirmos o número de elementos abertos pelo algoritmo; se dispuséssemos de um limitante superior $M^{*}$ do valor ótimo do problema, isto é, tal que $M^{*} \geqq g(p)$, ao fecharmos o nó $j_{k}$ na iteração $k$, só abriríamos os sucessores de $j_{k}$ cujos rótulos $f^{k}(j) \leqq M^{*}$ pois os demais não conduziriam a caminhos mínimos devido ao fato de estarmos considerando apenas grafos com custos não negativos. Esse procedimento, no entanto, só melhora o desempenho do algoritmo em termos de espaço utilizado e não em relação a número de fechamentos pois o algoritmo só fecha nós que podem eventualmente fazer parte da solução ótima do problema.

\section{PROPOSIÇÃO 2.1.10}

Seja $k^{*}$ a iteração na qual o algoritmo termina.

Sendo $\left\{j_{k}\right\}_{k=1}^{k^{*}}$ a seqüêencia de nós fechados pelo algoritmo de Dijkstra, tem-se que $\left\{f^{k}\left(j_{k}\right)\right\}_{k=1}^{k^{*}}$ é não decrescente e $f^{k}(\cdot)$ satisfaz $f^{k}(j) \leqq g(p)$ $\forall j \in C^{k}, \forall k \leqq k$ onde $k=\min \left\{k \in\left\{1, \ldots, k^{*}\right\} \mid p \in C^{k}\right\}$.

\section{Demonstração}


A demonstração se processa em duas etapas:

i) $\left\{f^{k}\left(j_{k}\right)\right\}_{k=1}^{k^{*}}$ é não decrescente

Seja $k \in\left\{1,2, \ldots, k^{*}-1\right\}$ e considere $f^{k}\left(j_{k}\right)$.

Se $f^{k+1}\left(j_{k+1}\right)=f^{k}\left(j_{k+1}\right)$, do critério de escollha de nós para fechamento, $f^{k}\left(j_{k+1}\right)=f^{k+1}\left(j_{k+1}\right) \geqq f^{k+1}\left(j_{k}\right)$.

Se $f^{k+1}\left(j_{k+1}\right) \neq f^{k}\left(j_{k+1}\right)$ então $f^{k+1}\left(j_{k+1}\right)=f^{k}\left(j_{k}\right)+c\left(j_{k}, j_{k+1}\right)$ $\geqq f^{k}\left(j_{k}\right)$ pois $c\left(j_{k}, j_{k+1}\right) \geqq 0$

Assim, $f^{k+1}\left(j_{k+1}\right) \geqq f^{k}\left(j_{k}\right)$ e a sequiência é não decrescente.

ii) $\forall j \in C^{k} f^{k}(j) \leqq g(p) \quad \forall k \mid p \notin C^{k}$

Como a escolha para fechamento fornece uma seqüência não decrescente $\left\{f^{k}\left(j_{k}\right)\right\}_{k \in N}$ temos que sendo $k_{1}$ e $k_{2}$ as iterações em que foram fechados os nós 1 e $p$ respectivamente, tem-se

$$
f^{k_{1}}(j) \leqq f^{k_{2}}(p)=g(p)
$$

e segue a afirmação.

Uma observação interessante na linha deste resultado é a seguinte: se considerarmos a seqüência de funções definidas em $N,\left\{\pi^{k}(\cdot)\right\}_{k \in N}$ onde

$$
\begin{gathered}
\pi^{k}: N \rightarrow \overline{\mathbb{R}} \\
\pi^{k}(j)=f^{k}(j)
\end{gathered}
$$

é claro que $\forall j \in N \quad\left\{\pi^{k}(j)\right\}_{k \in N}$ é não decrescente do critério de atualização dos rótulos $f^{k}(j)$.

Essa seqüência de funçôes, conforme veremos oportunamente permite que 0 algoritmo possa ser considerado um caso particular de uma outra classe de algoritmos conhecida como métodos de incremento, que se aplicam à resolução de problemas mais gerais do que o problema de determinação caminhos mínimos em grafos.

Esse conjunto de resultados que estudamos mostram a validade do algoritmo de Dijkstra para o caso de grafos finitos. No caso de grafos localmente finitos, isto $e$, tal que $\# \operatorname{suc}(j)<\infty \forall j \in N$, o algoritmo 
também é válido, desde que possamos garantir que apenas um número finito de nós pode ser fechado pelo algoritmo. Esse caso ocorre, por exemplo, quando $\exists N^{\prime} \subset N, N^{\prime}$ finito, tal que $\forall j \in N \backslash N^{\prime} g(j)>g(p)$. Nessa situação, $\forall j \in N \backslash N^{\prime} j \notin C^{k} \forall k \in N \mid p \notin C^{k}$ pelo resultado anterior.(que pode ser provado independentemente da finitude do grafo) ou tal forma que após um número finito de passos $p \in C^{k} \mathrm{e} o$ algoritmo pode terminar.

É importante salientar que tanto neste caso como no de grafos finitos a hipótese de custos nã̃o negativos é determinante para que se verifique a validade do processo, assegurando que qualquer nó atingível com custo inferior a $g(p)$ é fechado, com rótulo igual ao custo do caminho mínimo entre origem e o nó.

Uma das razóes pelas quais o algoritmo de Dijkstra tem sido estudado se deve à sua medida de complexidade computacional. Se utilizarmos o algoritmo simplex aplicado a, redes, em uma análise de pior caso sua complexidade é da ordem $O\left(2^{p}\right)$ com $p=\# N$ [Cu79] Dijkstra por sua vez apresenta complexidade polinomial conforme mostra o seguinte resultado

TEOREMA 2.1.11 (artigo 19)

Nas condições do teorema anterior o algoritmo de Dijkstra possue rapider pessimista * da ordem de $O\left(p^{2}\right)(p=\# N)$.

\section{Demonstração}

Para cada elemento $j \in O^{k}$ que 0 algoritmo escolhe para fechamento, são realizados no máximo $(p-1)$ comparaçōes para alteração dos rótulos, correspondendo à pesquisa nos elementos de $\operatorname{suc}(j)$.

Como no pior caso, todos os $p$ nós são fechados, poderemos efetuar $p *(p-1)$ comparaçốes e sendo o processo de inicialização e o de detecção de caminhos é, no máximo, buscar com o número de nós, a complexidade do algoritmo é dada pelo termo dominante $p *(p-1)$, ou seja, da ordem $O\left(p^{2}\right)$.

* Ver Terada[Te82], onde o conceito de complexidade é bem denominado rapidez pessimista, por corresponder a uma análise de pior caso 
$O$ fato de termos obtido um algoritmo polinomial em $p(p=\# N)$ não significa que não exista algoritmos com rapidez $O(\# E)$, ainda que não se conheça nenhum algoritmo com essa complexidade. Veja que $O(\# E)$ é um limitante inferior (em análise de pior caso ) da complexidade para a resolução do problema de existência de um caminho entre os nós 1 e $p$.

A desvantagem do algoritmo de Dijkstra se refere ao fato de ser válido apenas para grafos com custos não negativos, hipótese esta utilizada para garantir que ao fecharmos um nó determinamos um caminho mínimo da origem até ele. Isso sugere uma linha mais ampla de trabalho, permitindo que alguns custos sejam negativos, o que nos forçaria a considerar a possibilidade de que os nós sejam reabertos.

Para extendermos esses resultados uma possível ferramenta seria a de dualidade. Já havíamos visto que um candidato natural à variável dual b́tima era dado pelo custo do caminho mínimo da origem até cada um dos nós do grafo. Para os elementos $i \in N$ fechados pelo algoritmo tem-se que $f^{k}(i)=g(i)$ e portanto se pudermos fechar todos os nós, obtemos uma solução dual ótima dada por $u^{k} \in \mathbb{R}^{p} \quad u_{i}^{k}=-\mathbf{f}^{\mathbf{k}}$ (i) $\forall i \in N$.

Considerando-se então o algoritmo de Dijkstra para o caso de grafos finitos, e adotando como critério de parada, $O^{k} \neq \emptyset$ temos que ao final do processo todos os nós $j \in N$ atingíveis a partir da origem, estarão fechados e portanto com valor $f(j)=g(j)$. Assim, caso todos os nós sejam atingíveis temos :

\section{TEOREMA 2.1.12}

Seja $G \equiv(N, E)$ um grafo orientado sob as HIPÓTESES 1.1.2 e 2.1.1 e a HIPÓTESE BASICA.

Se na iteração $k^{*}$ na qual o algoritmo termina, $C^{k^{*}}=N$ então uma solução ótima para o problema dual (PMINC) é dada por $u^{*} \in \mathbb{R}^{\# N}$, $u_{j}^{k^{*}}=-f^{k^{*}}(j) \forall j \in N$

\section{Demonstração}


Como ao final do processo, $\forall j \in C^{k^{*}} f^{k^{*}}(j)=g^{k^{*}}(j)=g(j)$, segue do TEOREMA 1.2.4 (construção de variáveis duais) que $u^{*} \in \mathbb{R}^{\# N} u_{j}^{k}=$ $-\mathrm{f}^{\mathrm{k}}(\mathrm{j})=-\mathrm{g}(\mathrm{j})$ é uma solução ótima de (DMINC).

De certa forma Dijkstra pode ser entấo considerado um algoritmo para o dual (DMINC), no sentido de construir uma solução ótima para o mesmo, através da função $f^{k^{*}}(\cdot)$. Conforme veremos posteriormente, outros algoritmos podem ter o mesmo tipo de caracterização, ao tomarmos como candidato a variável dual a função que determina a escolha de nós para fechamento.

Se por um lado o algoritmo de Dijkstra é eficiente, em contrapartida restringe a classe de problemas aos quais se aplica e é claro que qualquer esforço no sentido de extender o algoritmo a problemas com custos reais pode acarretar alguma perda em termos de eficiência. Duas idéias fundamentais associadas ao algoritmo de Dijkstra podem ser exploradas na tentativa de resolver problemas com custos negativos. A primeira delas explora basicamente os conceitos de dualidade, procurando obter iterativamente viabilidade ao problema dual; a segunda consiste em um processo de rotulação de nós que independe da não negatividade dos custos : visando a construção da solução ótima.

Estudemos primeiramente um método que utiliza dualidade.

\section{2 - O MÉTODO DE FORD E DUALIDADE}

Seguindo as linhas propostas vamos primeiramente extender os princípios de dualidade implícitos no algoritmo de Dijkstra para o caso de grafos com custos reais.

A única restrição que iremos impor sobre os custos é a inexistência de circuitos de custo negativo, isto é, adotaríamos a seguinte hipótese : 
Para o grafo $G \equiv(N, E)$ considerado, qualquer que seja o circuito $C^{k}=\left(i_{1}, i_{2}, \ldots, i_{i}, i_{1}\right)$, o seu custo é não negativo $\left(c\left(C^{k}\right) \geqq 0\right)$.

Conforme estudamos, o algoritmo de Dijkstra constrói dois conjuntos $\left(C^{k}\right.$ e $\left.O^{k}\right)$ cujos rótulos $f^{k}(j)$ correspondem a custos de passeios entre a origem e os nós, sendo que ao final do processo, caso $C^{k^{*}}=N$, podemos determinar uma solução ótima dual, $u^{k} \in \mathbb{R}^{p} \quad u_{i}^{k}=f^{k^{*}}$ (i) $\forall i \in N$. Se a cada iteração definirmos um elemento $u^{k} \in \mathbb{R}^{p}$ com $u_{i}^{k}=-f^{k}(i)$, teríamos que $\forall(i, j) \in E$ tal que $i \in C^{k} u_{i}^{k}-u_{j}^{k}-f^{k}(i)+f^{k}(j)=-g(i)+g(j) \leqq c(i, j)$

Ou seja, para arcos $(i, j)$ com $i \in C^{k}$, a correspondente restrição no problema dual era satisfeita pelo vetor $u^{k}$, sendo atrvés de uma escolha bem determinada de nós, a obtenção de $u^{*}$ viável dual (se possível) era feita com complexidade polinomial (o que se deve basicamente a trabalharmos com custos não negativos).

Ao utilizarmos esse mesmo princípio de construção de elementos viáveis no problema dual para o caso em que permitimos a existência de custos negativos, vamos obter um método para a resolução do problema do caminho mais curto conhecido como MÉTODO DE FORD. A descriçâo que passamos a fazer deste método baseia-se nos trabalhos de [Si68] e [La76].

\section{MÉTODO 2.2.2 - O MÉTODO DE FORD}

$$
\begin{aligned}
& \llbracket f^{0}(1):=0 \\
& f^{0}(j):=-\infty ; \quad \forall j \in N \backslash\{1\} * \\
& k:=0 \text {; } \\
& \text { Enquanto } \exists(i, j) \in E \mid f^{k}(i)-f^{k}(j)>c(i, j) f(i) \in \mathbb{R} \text { faca } \\
& \left|\operatorname{Seja}_{j k} \in N\right| f^{k}\left(j_{k}\right) \in \mathbf{R} \\
& \text { Para cada } j \in \operatorname{suc}\left(j_{k}\right) \text { faca } \\
& \text { se } f^{k}\left(j_{k}\right)-f^{k}(j)>c\left(j_{k}, j\right) \\
& \text { entäo } f^{k+1}(j):=f^{k}\left(j_{k}\right)-c\left(\dot{j}_{k}, j\right) \\
& \text { senäo } f^{k+1}(j):=f^{k}(j)
\end{aligned}
$$

* Assim como no caso de Dijkstra poderíamos tomar inicialmente $f^{0}(j)=-m$, onde $m \mathbb{R}$ é um número suficientemente grande, $(m \geq g(j) \forall j \in$ $N \mid g(j) \in \mathbb{R}$ ), sem que isso afetasse os resultados que serão verificados. 


$$
\begin{aligned}
& \forall j \in N \backslash \operatorname{suc}\left(j_{k}\right) f^{k+1}(j):=f^{k}(j) \\
& k:=k+1_{i} \rrbracket
\end{aligned}
$$

\section{FIM-MÉTODO}

Observe que neste caso há um alto grau de indeterminação no método, uma vez que não foi especificado um critério, ainda que geral, de escolha do nó $j_{k}$ para alteração dos rótulos dos nós $j \in \operatorname{suc}\left(j_{k}\right)$.

$\mathrm{Na}$ verdade, é essa escolha que determina a eficiência do método : o algoritmo de Dijkstra pode ser entendido como um caso particular deste método. No sentido de procurar obter viabilidade dual, que, devido a uma boa escolha de nós, permitida pela não negatividade dos custos, possue complexidade polinomial.

Apesar da indeterminação de Ford, alguns aspectos são bastante relevantes com relação ao método.

\section{LEMA 2.2.3}

Seja $k$ uma iteração do método de Ford, e $j \in N$.

Se $f^{k}(j) \neq f^{k+1}(j)$ então $f^{k+1}(j)>f^{k}(j)$

Demonstração

Como $f^{k}(j) \neq f^{k+1}(j)$, para algum $j_{k} \in N, f^{k}\left(j_{k}\right)-f^{k}(j)>$ $c\left(j_{k}, j\right)$ e dá $f^{k+1}(j)=f^{k}\left(j_{k}\right)-c\left(j_{k}, j\right)>f^{k}(j)$.

Um resultado bastante importante é o seguinte:

\section{PROPOSIÇÃO 2.2.4}

\section{Seja $k \in N$}

Então $\forall j \in N$, ou $f^{k}(j)=-\infty$ ou existe um caminho $P=$ $\left(i_{1}, i_{2}, \ldots, i_{i}\right)$ entre $i_{1}=1$ e $i_{i}=j$ tal que $f^{k}\left(i_{r}\right)=-c\left(P_{r}\right) \forall r \in\{1,2, \ldots, t\}$ onde $P_{r}=\left(i_{1}, \ldots, i_{r}\right)$ 


\section{Demonstração}

Por indução sobre o número de iterações

Base de indução

Se $k=0$ a afirmação é trivial

Se $k=1$ temos

$$
\text { i) se } j \notin O^{1} \cup C^{1}=\{1\} \cup \operatorname{suc}\{1\} \text { então } f^{k}(j)=-\infty
$$

ii) $j=1 f^{1}(1)=0$ e tomo $P=(1)$

ii) se $j \in \operatorname{suc}(a)$ tomando $P=(1, j)$ temos $f^{1}(1)=0$

$f^{1}(j)=c(P)$ e a afirmação segue trivialmente observando que $j \neq 1$ pois o grafo näo possui laços.

\section{Hipótese de indução}

Suponhamos que $\forall k \quad 1 \leq k \leq p-1$ a afirmação seja verdadeira, isto é, $\forall j \in N$ ou $f^{k}(j)=-\infty$ ou $\bar{\exists} P^{\bar{k}}=\left(i_{1}, \ldots, i_{s}\right)$ um caminho entre $i_{1}=1$ $\left.\mathrm{e} i_{s}=j \operatorname{com} f^{k}\left(i_{i}\right)=c\left(P^{k}\right) \forall l \in\{1,2, \ldots, 8\} \quad P_{l}^{k}=\left(i_{1}, i_{2}, \ldots, i_{l}\right)\right)$ $k=p$

Seja $j \in N$ qualquer

Se $f^{k}(j)=-\infty$ nada a fazer

Caso contrário a demonstração se processa por etapas.

Mostremos inicialmente que o caminho correspondente ao nó $j_{k}$ não foi alterado.

De fato, seja $P=\left(i_{1}, i_{2}, \ldots, i_{t}\right)$ o caminho associado a $j_{k}$ na iteração $k-1$ e $l_{0}$ maior índice para o qual $f^{k}(i \tau) \neq f^{k-1}(i \tau)(\bar{l} \in\{1,2, \ldots, t\})$.

Do critério de al teração dos rótulos temos que $i_{\tau} \in \operatorname{suc}\left(j_{k}\right)$ e $f^{k}\left(i_{\tau}\right)=$ $f^{k-1}\left(j_{k}\right)-c\left(j_{k}, i_{i}\right)>f^{k-1}\left(i_{i}\right)$

Como $f^{k-1}\left(j_{k}\right)=f^{k-1}\left(i_{i}\right)-\sum_{m=l+1}^{t-1} c\left(i_{m}, i_{m+1}\right)$ da hipótese de indução, segue que $f^{k}\left(i_{\eta}\right)=f^{k-1}\left(i_{l}\right)-\sum_{m=l+1}^{t-1} c\left(i_{m}, i_{m+1}\right)-c\left(j_{k}, i_{l}\right)>$ $f^{k-1}\left(i_{\tau}\right)$

Assim, $-\sum_{m=l+1}^{t-1} c\left(i_{m}, i_{m+1}\right)-c\left(j_{k}, i_{i}\right)>0$, ou numa notação simplificada, $c\left(i_{\tau_{1}} i_{l+1}\right)+c\left(i_{l_{+1}}, i_{I_{+2}}\right)+\ldots+c\left(i_{-1}, j_{k}\right)+c\left(j_{k}, i_{i}\right)<0$ ou, seja existe um circuito de custo negativo, o que contraria a hipótese originalmente assumida. Assim, $\forall m \quad 1 \leqq m \leqq t \quad f^{k-1}\left(i_{n}\right)=f^{k}\left(i_{n}\right)$, ou seja 0 caminho não teve seus rótulos alterados.

Seja $j \in N \backslash\left\{j_{k}\right\}$ um nó qualquer com $f^{k}(j) \in \mathbb{R}$. Temos dois casos a estudar: 
i) Se $j \in \operatorname{suc}\left(j_{k}\right)$, tomamos o passeio $P=\left(i_{1}, i_{2}, \ldots, i_{t}, j\right)$. Esse passeio é um caminho pois caso contrário detectaríamos um circuito de custo negativo. Além disso é imediato que $f^{k}(j)=c(P)$ e $f^{k}\left(i_{l}\right)=$ $c\left(P_{l}\right) \quad \forall l \in\{1,2, \ldots, t\}$

ii) Se $j \notin \operatorname{suc}\left(j_{k}\right)$, tomando-se o caminho $P^{k-1}=$ $\left(n_{1}, n_{2}, \ldots, n_{r}\right)$ entre $n_{1}=1$ e $n_{r}=j$ associado a $j$ na iteração $k-1$, temos $f^{k-1}\left(n_{l}\right)=c(P) \quad \forall l \in\{1,2, \ldots, \tau\}$ pela hipótese de indução.

Seja então $l$ o maior índice para o qual $f^{k}\left(i_{i}\right) \neq f^{k-1}\left(i_{i}\right)$

Tomando-se $P^{k}=\left(i_{1}, i_{2}, \ldots, i_{t}, n_{l+1}, \ldots, n_{r}\right)$ a afirmação segue da hipótese de indução e pela inexistência de circuitos de custo negativo.

Do princípio de indução segue a tese.

Observe que esse resultado juntamente com 0 anterior nos assegura que as alterações de rótulos efetuadas buscam caminhos de custo cada vez menor, mos trando que Ford pesquisa o grafo na "direção correta". Assim, como em Dijkstra temos :

\section{COROLÁRIO 2.2.5}

Seja $j \in N$

Então $\forall j \in N \quad f^{k}(j) \geqq g(j)$

Além disso

\section{COROLÁRIO 2.2.6}

Seja $k \in N$

Se $\exists k \in N$ tal que $f^{k}(j) \in \mathbb{R}$ então $j$ é atingível a partir da origem.

Mais ainda, assim como em Dijkstra, ao obter custos de caminhos mínimos não mais altera os correspondentes rótulos.

\section{LEMA 2.2.7}

Seja $k^{*} \in N$ tal que $f^{k^{*}}(j)=-g(j)$, para algum $j \in N$ Então $\forall k \geq k^{*} f^{*}(j)=-g(j)$ 


\section{Demonstração}

Se $g(j) \in \mathbb{R}$ então como da PROPOSIÇÃO 2.2.4, $-f^{k}(j)$ corresponde ao custo de um caminho entre 1 e $j, \forall k \geqq k^{*}$ temos que $-f^{k}(j) \geqq-$ $f^{k^{*}}(j)=g(j)$ e do LEMA 2.2.4 segue a afirmação, pois para que $f^{k}(j) \neq$ $-g(j)$ deveríamos ter $f^{k}(j) \in \mathbb{R}$ e portanto $j$ seria atingível a partir da origem.

Ao determinar o custo de um caminho mínimo entre a origem $e$ um dado nó podemos então garantir que

\section{LEMA 2.2.8}

Seja $k^{*} \in N$ e $i \in N$ tais que $f^{k^{*}}(i)=-g(i)$

Então $\forall j \in \operatorname{suc}(i) \quad \forall k \geqq k^{*} f^{k}(i)-f^{k}(j) \leqq c(i, j)$

\section{Demonstração}

Assumamos sem perda de generalidade que na iteração $k^{*}$ o nó $i$ receba o rótulo $f^{k^{*}}(i)=-g(i)$ Temos então que $-f^{k^{*}}(j) \leqq c(i, j)+f^{k^{*}}(i)$ do critério de alteração de rótulos é como o valor de $-f^{k}(j)$ só pode decrescer, e $\forall k \geqq k^{*} f^{k}(i)=f^{k^{\prime}}(i)$ vem que $\forall k \geqq k^{\prime}-f^{k}(j)(i, j)+f^{k}(i)$.

Assim , a termos detectado o custo de um caminho mínimo entre a origem e um dado nó não mais é escolhido para atualização de rótulos de seus suces sores. Logo, se dispusermos de um critério razoável de escolha de nós tem-se

\section{TEOREMA 2.2.9}

Seja $G \equiv(N, E)$ um grafo orientado satisfazendo as HIPBTESES 1.1.2, 2.2.2 e a HIPÓTESE BÁSICA. 
Então o método de Ford, quando aplicado a $G$ termina em um número finito $k^{*}$ de passos.

\section{Demonstração}

Como 0 grafo não possui circuitos de custo negativo e é finito, temos que para cada nó $j \in N$ é finito o número de caminhos entre 1 e $j$.

Como as alterações dos rótulos de um dado nó $j$, correspondem á determinação de caminhos entre 1 e $j$ de custo cada vez menor, o número de alteraçốes possíveis é finito.

Mas ao obtermos $f^{k^{*}}(j)=-g(j)$ esse valor não é mais alterado ( e do resultado anterior não mais é escolhido para atualização pois $f^{k}(j)-$ $\left.f^{k}(l)(j, l) \quad \forall l \in N:(j, l) \in E\right)$. Assim, como o método escolhe a cada passo, um elemento para alteração, da finitude do grafo segue a afirmação.

Neste caso, contrariamente ao algoritmo de Dijkstra não podemos determinar a priori um subconjunto de nós cujos rótulos $f^{k}(j)$ correspondam ao custo de um caminho mínimo. (Naquele caso um subconjunto com essa propriedade era dado pelos nós fechados). Isso só será assegurado no término do algoritmo conforme mostra o seguinte resultado.

\section{PROPOSIÇÃO 2.2.10}

Se ao final da iteração $k^{*}$ o método de Ford termina então $\forall j \in$ $N \mid f^{k^{*}}(j) \in \mathbb{R}$ tem-se $f^{k^{*}}(j)=-g(j)$

\section{Demonstração}

Seja $j \in N \mid f^{k^{*}}(j) \in \mathbb{R}$ e considere $P^{k}=\left(i_{1}, \ldots, i_{t}\right)$ um caminho entre $i_{1}=1 \mathrm{e} i_{t}=j$.

Claramente nessa iteracão $f^{k^{*}}\left(i_{l}\right)-f^{k^{*}}\left(i_{i+1}\right) \leqq c\left(i_{i}, i_{l+1}\right) \quad \forall l \in$ $\{1,2, \ldots, t-1\}$ e portanto

$$
f^{k^{*}}\left(i_{l}\right)-f^{k^{*}}\left(i_{i}\right)=\sum_{l=1}^{t-1}\left(f^{k^{*}}\left(i_{l}\right)-f^{k^{*}}\left(i_{l+1}\right) \leqq \sum_{i=1}^{t-1} c\left(i_{l}, i_{l+1}\right)=c\left(P^{*}\right)=g(j)\right.
$$


Como o grafo não possue ciclos de custo negativo temos então $f^{k^{*}}\left(i_{1}\right) \geqq g\left(i_{1}\right)=g(1) \geqq 0$ e portanto $-f^{k^{*}}\left(i_{t}\right) \leqq f^{k^{*}}\left(i_{1}\right)-f^{k^{*}}\left(i_{t}\right) \leqq g(j)$

Mas $-f^{k^{*}}\left(i_{i}\right)=-f^{k^{*}}(j)$ corresponde ao custo de um passejo e portanto $-f^{k^{\circ}}(j)=g(j)$ e segue a tese.

Podemos então verificar que 0 método não termina enquanto não detectar para cada nó atingível a partir da origem, o custo de um caminho mínimo entre a origem e esse nó.

\section{COROLÁRIO 2.2.11}

Seja $k \in N$ uma iteração do método de Ford e $j \in N$ um nó atingível a partir de 1.

Se $f^{k}(\hat{j}) \neq-g(j)$ então $\exists(\hat{i}, \hat{j}) \in E \mid f^{k}(\hat{i})-f^{k}(\hat{j})>c(\hat{i}, \hat{j})$ (isto é o algoritmo não termina nesta iteração)

Demonstração

Trivial.

Ao final do processo um caminho mínimo entre a origem e cada nó atingí vel pode ser determinado:

\section{TEOREMA 2.2.12}

Se o método de Ford termina em um número finito $k^{*}$ de passos então $\forall j \in N$ ou $f^{k^{*}}(j)=-\infty$, ou existe um caminho mínimo $P^{k}=$ $\left(i_{1}, i_{2}, \ldots, i_{t}\right)$ entre $i_{1}=1$ e $i_{t}=j$ de custo $f^{k^{*}}(j)$ tal que $f^{k^{*}}\left(i_{l}\right)=-g\left(i_{l}\right)$ $\forall l \in\{1,2, \ldots, t\}$

\section{Demonstração}

$\operatorname{Seja} j \in N$ 

a) $f^{k^{*}}(j)=+\infty$ nada a fazer
b) $f^{k^{*}}(j) \in \mathbb{R}$

Nesse caso, claramente $f^{k^{*}}(j)=-g(j)$ e tomando-se um caminho mínimo entre 1 e $j, P^{k}=\left(i_{1}, i_{2}, \ldots, i_{l}\right)$ é claro que $f^{k^{*}}(j)=-g(j)$ e segue a afirmação.

Note que para este método a seqüência $\left\{f^{k}\left(j_{k}\right)\right\}_{k \in N}$ não é necessariamente crescente, uma vez que a escolha deste elemento é numa certa medida arbitrária como mostra o seguinte exemplo:

EXEMPLO 2.2.13

Considere o grafo $G \equiv(N, E)$ dado por

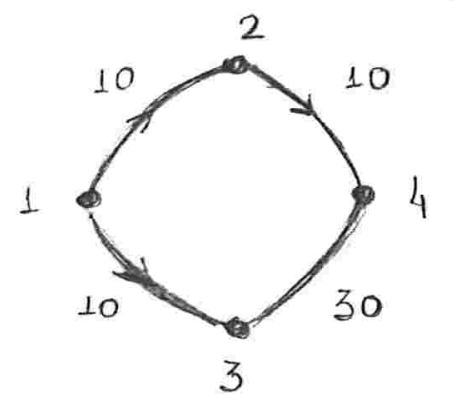

$$
\begin{gathered}
N=\{1,2,3,4\} \\
E=\{(1,2),(1,3),(2,4),(3,4)\}
\end{gathered}
$$

Para a escolha de nós abaixo teremos

$\begin{array}{lll}k & j_{k} & f^{k}\left(j_{k}\right) \\ 0 & 1 & 0 \\ 1 & 3 & -10 \\ 2 & 4 & -40 \\ 3 & 2 & -10 \\ 4 & 4 & -20\end{array}$

que nos mostra que $\left\{f^{k}\left(j_{k}\right)\right\}_{k \in N}$ não é crescente (näo decrescente) nem tampouco decrescente (não crescente). 
Apesar de não podermos assegurar sequer que essa seqüência seja não decrescente, se tomarmos a seqüência de funções $\left\{\pi^{k}(\cdot)\right\}_{k \in N}$, onde

$$
\begin{gathered}
\pi^{k}: N \rightarrow \overline{\mathbb{R}} \\
\pi^{k}(i)=-f^{k}(i)
\end{gathered}
$$

é claro, pelo LEMA 2.2.3 que $\forall j \in N\left\{\pi^{k}(\cdot)\right\}_{k \in N}$ é não decrescente,

e portanto o método constrói implicitamente uma seqüência de funções não decrescentes

Em termos da notação adotada no capítulo precedente teríamos que

$$
\pi^{k}(j)= \begin{cases}\pi^{k}: N \rightarrow \overline{\mathbb{R}} \\ \infty c, x\rangle & \text { para algum } x \in X^{1 j} \text { se } j \in O^{k} \cup C^{k} \\ \infty & \text { caso contrário }\end{cases}
$$

( $X^{1 j}$, conforme TEOREMA 1.1.11)

Isso permite que, assim como no caso de Dijkstra, o método de Ford seja aplicável a problemas mais gerais que o problema de busca em grafos.

É interessante observar que o método mantém uma estrita relação com o algoritmo simplex. Neste último caso mantem-se, a cada passo, viabilidade primal e folgas complementares, buscando atingir viabilidade dual. Considerando-se um problema na forma $\min _{x \in X}\langle c, x\rangle \quad X=\{x \in$ $\left.\mathbb{R}^{\mathrm{q}} \mid \mathrm{Ax}=\mathrm{bx} \geq 0\right\}$ viabilidade dual corresponde a obter $c-A^{T} u \geqq 0$, sendo que caso $\left(c-\bar{A}^{T} u\right)_{i}<0$ pode-se crescer a correspondente componente $x_{i}$ obtendo-se então uma diminuição do valor da função objetivo. Ao trabalharmos com o método de Ford utilizamos um princípio semelhante, uma vez que viabilidade dual corresponde a termos $c(i, j)-u_{i}: u_{j} \geqq 0 \quad \forall(i, j) \in E$ e caso isto näo ocorra (isto é, $\exists(\hat{i}, \hat{j}) \in E\left(c(\hat{i}, \hat{j})-u_{\hat{i}}+u_{\hat{j}}<0\right)$ alterase a varíavel dual $u$, de forma a se obter $c(i, j)-u_{i}+u_{j}=0$ podendo-se então "crescer" a correspondente variável primal $x_{i j}$ sem que se perca folgas complementares.

Com relação a dualidade vale também o seguinte resultado 
Seja $G \equiv(N, E)$ um grafo sob as HIPßTESES 1.1.2, 2.2.1 e HIPÓTESE BÁSICA

Então, se ao final de $k^{*}$ iteraçóes o método termina e $f^{k^{*}}(j) \in$ $\mathbb{R} \forall j \in N$ então uma solução do problema dual (DMINC) é dada por $u^{k} \in \mathbb{R}^{\# N}, u_{j}^{k}=\mathrm{f}^{\mathbf{k}^{*}}(\mathrm{j}) \forall \mathrm{j} \in \mathrm{N}$

\section{Demonstração}

Claramente, ao final do processo, $\forall(i, j) \in E f^{k^{*}}(i) \leqq f^{k^{*}}(j) \leqq c(i, j)$ e $u^{k}$ é viável. Além disso, como o método só termina ao determinar o custo do caminho mínimo entre a origem e cado nò atingível, sendo $f^{k^{*}}(p) \in \mathbb{R}$, vem que $f^{*}(p)=-g(p)$. Mas $f^{*}(1)=0$ e portanto $u_{1}^{*}-u_{p}^{*}=0+g(p)=$ $g(p)$, donde segue que $u^{*}$ é solução ótima do problema.

Admitindo-se que o método termine, seu desempenho não é em linhas gerais tão bom quanto o de Dijkstra. É claro que o fator que determina a complexidade do método de Ford é a escolha do nó $j_{k}$ cujos sucessores terão seus rótulos alterados; particulares processos de alteração de rótulos podem conduzir a diferentes graus de complexidade.

No trabalho de Lawler [La.54] vamos encontrar casos em que particulares do algoritmo de Ford, com diferentes graus de complexidade, cabendo destacar por exemplo o algoritmo de Bellman-Ford cuja complexidade é da ordem $O\left(p^{3}\right)$.

A diferença essencial entre os métodos de Ford e Dijkstra é que não possui ciclos de custo negativo, sem possuir uma boa regra de escolha de nós para pesquisa que indique a proximidade do alvo, enquanto que Dijkstra, por trabalhar com custos não negativos escolhe os nós mais "promissores" para pesquisa. 0 algoritmo de Dijkstra, garante devido á não negatividade dos custos, que o custo do caminho percorrido para atingir um nó fechado qualquer, é um subestimador do custo do caminho mínimo entre a origem e 0 alvo; isso ocorre basicamente pelo fato de que o algoritmo dispóe de alguma informação quanto ao "custo" para se atingir o alvo, qual seja, que seu valor é não negativo. No caso de Ford, ainda que saibamos que aos rótulos $f^{k}(j)$ cujo valor seja real temos associado o custo de um caminho, nada se pode afirmar quanto à sua relaçao com a solução do problema pois o custo para se atingir o alvo a partir de um dado nó pode ser negativo e portanto $f^{k}(j)$ não subestima o valor ótimo do problema. 
Essas considerações nos mostram que o algorimo de Dijkstra de certa forma se utiliza de alguma informação quanto à proximidade do alvo, o que permite que a pesquisa de nós por ele efetuada seja bem determinado. Ora, se para o caso de grafos com custos negativos também dispusermos de algum tipo de informaçấo sobre o "futuro", isto é, se tivermos algum tipo de indicação quanto à proximidade do alvo, talvez possamos obter algoritmos "eficientes".

Vamos então prosseguir nessa linha estudando algoritmos que, assim como Dijkstra, rotulam os nós do grafo utilizando alguma informação quanto à proximidade do alvo. 


\section{Capitulo 3}

\section{$\mathbf{O}$ algoritmo $\mathbf{A}$}

$\mathrm{O}$ algoritmo $\mathrm{A}$ [Ni71], bastante conhecido em inteligência artificia] e um método para a determinação de caminhos mínimos em grafos, que utiliza, para definir a sequência de deteç̧ôes de caminhos, alguma informação quanto a estrutura do problema modelado pelo grafo, a qual estima a proxi midade entre os nós e 0 alvo a ser atingido.

A função que estima distâncias no algoritmo $\mathrm{A}$ e chamada heurística, sendo o fator determinante para o bom desempenho do algoritmo.Em geral essa terminologia causa confusão pois algoritmos aplicados a particulares problemas e que utilizam alguma informação obtida empiricamente costumam ser denominados "algoritmos heurísticos", sendo que neste caso näo se pode provar a otimalidade da solução obtida.No nosso caso 
heurísticas são funçōes bem definidas,que permitem assegurar a validade do algoritmo.

$\mathrm{O}$ algoritmo pode ser entendido como uma generalização de $\mathrm{Di}$ jkstra, para o caso de grafos com custos reais,que mantém uma estreita relação com duali dade.

\section{ALGORITMO 3.1 - ALGORITMO A}

Assim como Dijkstra, o algoritmo A gera iterativamente dois subconjuntos de nós do grafo (abertos e fechados), associando rótulos a esses nós ,que permitem a identificação de caminhos da origem até eles.Aplicável a problemas com custos reais, o algoritmo admite a reabertura de nós incluidos em fechados.O critério de fechamento por sua vez,parte de uma particular função $\mathrm{f}($.$) definida sobre os nós do grafo,selecionando, dentre$ os elementos $j \in N$ abertos, aquele com menor valor $\mathrm{f}(\mathrm{j})$ para fechamento.

Observe que,colocado sob esta forma o método inclui algoritmos clássicos como o algoritmo de busca em profundidade, onde o elemento escolhido para fechamento e o de maior "profundidade" ,isto é, aquele mais recentemente incluido na lista de elementos abertos.

$\mathrm{O}$ algoritmo $\mathrm{A}$ utililiza um estimador aditivo, isto é, de uma função $f: N \rightarrow \mathbb{R}$, na forma $f(j)=\hat{g}(j)+\hat{h}(j), j \in N$,onde $\hat{g}(j)$ e $\hat{h}(j)$ correspondem respectivamente a uma super-estimativa do custo de um caminho mínimo entre j e o nó alvo (i.e., $\hat{h}(j)$ estima $k(j, p))$.Essa denominação é a utilizada por Nilsson [Ni81],sendo a nomenclatura $A^{*}$ reservada para o caso em que se impóe particulares propriedades a função $\hat{h}($. $)$.Dentro desta caracterização para cada nó $j \in N, f(j)$ corresponde a uma estimativa do custo de um caminho entre 1 e $p$, restrito a passar pelo nó j.Um estimador natural de $g(j)$ é dado então pelo custo do caminho encontrado no processo de busca,conforme os algoritmos de Dijkstra e Ford,por exemplo, o que implica $\hat{g}(j) \geqq g(j) \forall j \in N$.

Fixemos as hipóteses utilizadas:

\section{HIPóTESE 3.1.1}

Para o grafo $G \equiv(N, E)$ considerado, qualquer que seja o circuito $C^{*}=\left(\dot{i}_{1}, \dot{i}_{2}, . . i_{i}, i_{1}\right), c\left(C^{*}\right) \geqq 0$,isto é,o grafo não possue circuitos de custos negativos. 
Serão mantidas aqui também a hipótese básica assumida no capitulo 1 (o grafo e orientado, conexo e sem arcos "paralelos"), bem como a hipótese 1.1.2 (finitude do grafo), apesar de que o algoritmo A pode ser aplicado ao caso em que o grafo é localmente finito (i.e., tal que $\forall j \in N, \# \operatorname{suc}(j)<\infty)$, desde que se imponha hipóteses adicionais que assegurem que o número de aberturas e fechamentos do processo seja finito.

A notação que utilizaremos, apesar de complexa, permite que sejam verificados resultados cuja complexidade não fica em geral evidente.Assim como em Dijkstra, denotaremos os conjuntos de nós abertos e fechados por $O^{k}$ e $C^{k}$ respectivamente.Quanto aos rótulos associados aos nós ,consideraremos não somente o nó, mas também alguma informação que nos permita obter tais caminhos ao término do processo.Mais geralmente,definiremos uma rotulação no grafo como sendo uma aplicação

$$
r: N \rightarrow \mathbb{R x} \mathbb{N}\left(\mathbb{N}=\mathbb{N} \cup\{\lambda\}^{*}\right)
$$

$\mathrm{O}$ algoritmo de Dijkstra, conforme já estudamos, associa iterativamente aos nós, um elemento $f^{k}(j)$ que corresponde ao custo do "menor" caminho encontrado pelo algoritmo até aquela iteraçăo.Utilizando-se esse princípio,podemos associar aos nós um rotulação

$$
\begin{aligned}
& r: N \rightarrow \overline{\mathbb{R x}} \bar{N} \\
& r(j)=(\hat{g}(j), \hat{a}(j))
\end{aligned}
$$

onde $\hat{g}(j)$ e o custo de um caminho entre 1 e j construido pelo algoritmo, (ou $\hat{g}(j)=\infty$ se nenhum caminho entre 1 e j foi determinado) e cujo nó que antecede j no mesmo e $\hat{a}(j)$ (ou $\hat{a}(j)=\lambda)$.

Como esse caminho pode variar com as iteraçöes dos algoritmos, para que nossa notação seja consistente, esses rótulos serâo indexados com as iteraçöes.

Sendo $J^{k}=\left\{j \in N \mid\right.$ o algoritmo encontrou um caminho $P^{k}(j)$ entre $1 \mathrm{e} \mathbf{j}$ até a iteração $\mathbf{k}\}$

consideraremos $\left\{r^{k}(.)\right\}_{k \in \mathbb{N}}$,

$$
r^{k}: N \rightarrow \bar{R} \bar{x}
$$

* Entenda-se por $\lambda$ um elemento não pertencente a $N$, ou , aqueles familiarizados com as notaçōes clássicas de estruturas de dados,com 0 simbolo do elemento "vasio" 


$$
\begin{aligned}
& r^{k}(j)=\left(g^{k}(j), a^{k}(j)\right) \\
& \operatorname{com} a^{k}(.) \text { e } g^{k}(.) \text { tais que : } \\
& g^{k}(j)= \begin{cases}\infty & j \in N \backslash J^{k} \\
0 & j=1 \\
c\left(P^{k}(j)\right) & c c\end{cases} \\
& a^{k}(j)= \begin{cases}\lambda & j \in N \backslash J^{k} \\
1 & j=1 \\
\text { antecessordejnocaminhoP }(j) & c c\end{cases}
\end{aligned}
$$

Em termos da notação adotada,não construiremos os rótulos $r^{k}($. por estarem implicitamente definidos ao constrirmos os $a^{k}($.$) e g^{k}($.$) . Qua-$ nto a implementação, podemos considerar que os rótulos são elementos de uma lista ligada, sendo $a^{k}(j)$ um apontador para outro elemento da lista (eventualmente, $a^{k}(j)=\lambda$ ). Para que essa lista estivesse bem definida, a identificação do nó também deveria fazer parte da informaçäo,isto é , os rótulos deveriam ser da forma $\left(j, g^{k}(j), a^{k}(j)\right)$, porém isso sobrecarregaria ainda mais a notação utilizada.

\section{ALGORITMO 3.1.2}

Admitiremos que seja conhecida uma sequência $\left\{h^{k}(.)\right\}_{k \in \mathbb{N}}$ de funções definidas sobre os nós do grafo, que chamaremos de estimadores, sobre as quais näo e imposta a priori, nenhuma restrição.

$$
\begin{aligned}
& \text { O ALGORITMO A } \\
& \qquad g^{0}(1):=0 ; \\
& g^{0}(j):=\infty \forall j \in N \backslash\{1\} ; \\
& f^{0}(j):=g^{0}(j)+h^{0}(j) \forall j \in N ; \\
& a^{0}(1):=1 ; \\
& a^{0}(j):=\lambda \forall j \in N \backslash\{1\} ; \\
& O^{0}:=\{1\} ; \\
& C^{0}:=0 ; \\
& k:=0 ;
\end{aligned}
$$




$$
\begin{aligned}
& \text { Enquanto }\left(O^{k} \neq 0\right) \text { faça } \\
& \text { 『Seja } j_{k} \in \arg \min _{j \in O^{k}} f(x) \\
& F(k):=f^{k}\left(j_{k}\right) \text {; } \\
& O^{k+1}:=O^{k} \backslash\left\{j_{k}\right\} \\
& C^{k+1}:=C^{k} \cup\left\{j_{k}\right\} \\
& \text { Para cada } j \in \operatorname{suc}\left(j_{k}\right) \text { faça } \\
& \text { se } g_{k}(j)>g^{k}\left(j_{k}\right)+c\left(j_{k}, j\right) \\
& \text { então } \left.\llbracket g^{k+1}\right)^{1}:=g^{k}\left(j_{k}\right)+c\left(j_{k}, j\right) \text {; } \\
& a^{k}+j:=j k i \\
& O^{k+1}:=O^{k} \cup\{j\} \\
& \text { se } \mathrm{j} \in C^{k} \\
& \text { entäo } C^{k+1}:=C^{k+1} \cup\{j\} ; \\
& \text { senão }\left\lceil g^{k+1}(j):=g^{k}(j)\right. \\
& a^{k+1}(j):=a^{k}(j) ; \\
& f^{k+1}(j):=g^{k+1}(j)+h^{k+1}(j) \forall j \in N \text {; } \\
& a^{k+1}(j):=a^{k}(j) \forall j \in N \backslash \operatorname{suc}\left(j_{k}\right) ; \\
& g^{k+1}(j):=g^{k}(j) \forall j \in N \backslash \operatorname{suc}\left(j_{k}\right) ; \\
& k:=k+1 ;\rfloor
\end{aligned}
$$

Se $p \in C^{k-1}$ então recupere um caminho mínimo através da função $a^{k}($.$) , iniciando por a^{k}(p) . \unlhd$

Cometeremos aqui também, um certo abuso de notação, chamando - método de algoritmo, pois não definimos o critério de escolha para fecha. mento nos casos onde \# $\arg \min _{j \in O^{k}} f^{k}(j)>1$.

Basearemos a verificação da validade do algoritmo nos trabalhos de Gonzaga ([Go78]), Pearl ([Pe83]) e Nilsson ([Ni81] ,[Ni73]).

\section{DEFINIÇÄO 3.1 .3}

Dada uma função

$\varrho: N \cup\{\lambda\} \rightarrow \varrho: N \cup\{\lambda\}$ e $r \in \mathbb{N}$, denotamos por $\varrho_{r}($.$) a$

$\varrho_{r}: N \cup\{\lambda\} \rightarrow \varrho: N \cup\{\lambda\}$ 
$\varrho= \begin{cases}\lambda & \text { se } j=\lambda \\ j & \text { se } r=0 \\ e\left(e_{r-1}\right) & \text { ce }\end{cases}$

ou seja, $\ell_{r}($.$) corresponde a aplicação da função \varrho($.$) , r vezes$

Estamos interessados em $a_{r}^{k}().(r \in \mathbb{N})$, uma vez que $a^{k}(j)$ corresponde ao antecessor do nó $j$, associado a um particular caminho, e portanto sua aplicação sucessiva deve estar relacionada com a obtenção do mesmo.

FATO 3.1.4

$$
\begin{aligned}
& \forall k \in \mathbb{N} \quad \forall j \in O^{k} \cup C^{k}, \\
& \text { ou } j=\operatorname{louak}(j)=\lambda \\
& \text { ou } j \in \operatorname{suc}\left(a^{k}(j)\right) .
\end{aligned}
$$

Demonstração

Trivial por construção.

FATO 3.1.5

Sejam $k \in \mathbb{N}, j \in \mathbb{N} \backslash\left(O^{k} \cup \mathbf{C}^{k}\right)$.

Entäo $a^{k}(j)=\lambda$ e $g^{k}(j)=\infty$

Demonstração

Imediata.

FATO 3.1.6

Sejam $(\mathbf{k} 1, \mathbf{k} 2) \in \mathbb{N} \times \mathbb{N}$ e $j \in N$.

Se $a^{k 1}(j) \neq a^{k 2}(j)$ então $g^{k 1}(j) \neq g^{k 2}(j)$. 
Demonstração

Trivial

\section{LEMA 3.1.7}

Seja $\mathbf{k} \in \mathbb{N}, \mathbf{j} \in \mathrm{O}^{\mathbf{k}} \cup \mathbf{C}^{\mathbf{k}}$.

Então, $a^{k}(j) \neq \lambda$ e $g^{k}(j) \neq \infty$

Demonstração

Por indução sobre o número de iteraçōes.

$\mathrm{k}=0$

Claramente, $O^{0} \cup C^{0}=\{1\}$ e , por construção $a^{0}(1)=1 g^{0}(1)=0$.

Passo da Indução

Suponhamos que $\forall k 0 \leqq k \leqq s-1$, a afirmação seja verdadeira.

$k=s$

Seja $\mathrm{j} \in O^{k} \cup C^{k}$

Temos dois casos a analisar :

i.) $g^{k}(j)=g^{k-1}(j)$

Nesse caso, do processo de construção dos conjuntos $O^{k}$ e $C^{k}$, como $\mathrm{j} \in O^{k} \cup C^{k}$ e $g^{k}(j)=g^{k-1}(j), \mathrm{j} \in O^{k-1} \cup C^{k-1}$ e da hipótese de indução segue que $g^{k}(j) \in \mathbb{R}$.Como $g^{k}(j)=g^{k-1}(j)$, temos $a^{k}(j)=$ $a^{k-1}(j)$, e novamente da hipótese de indução vem que $a^{k}(j) \neq \lambda$.

ii.) $g^{k}(j) \neq g^{k-1}(j)$

Neste caso o rótulo de $\mathrm{j}$ foi alterado e temos $a^{k}(j)=j_{k} g^{k}(j)=$ $g^{k-1}(j)+c\left(j_{k}, j\right)$, onde $j_{k} \in O^{k-1}$ é o elemento escolhido para fechamento nessa iteração. Como $g^{k}\left(j_{k}\right) \in \mathbb{R}$, pela hipótese de indução, segue que $g^{k}(j) \in \mathbb{R}$. Além disso, $a^{k}(j)=j_{k} \neq \lambda$. 
Do princípio de indução segue a afirmação.

\section{COROLÁRIO 3.1.8}

Seja $(\mathrm{k}, \mathrm{r}) \in \mathbb{N} \times \mathbb{N} \mathbf{j} \in \mathrm{O}^{\mathrm{k}} \cup \mathrm{C}^{\mathrm{k}}$. Então $a_{r}^{k}(j) \neq \lambda$.

Demonstração

Trivial pelo lema anterior,observando que

$$
\forall j, a^{k}(j) \neq \lambda, a^{k}(j) \in C^{k} \cup O^{k} \text {. }
$$

Nessas condições vem que :

\section{COROLÁRIO 3.1.9}

Seja $k \in \mathbb{N}, j \in O^{k} \cup C^{k}$. Então $\forall r \in \mathbb{N} \quad a_{r}^{k}(j) \in O^{k} \cup C^{k}$

Demonstração

Do resultado anterior $, \forall r \in \mathbb{N}, a_{r}^{k}(j) \neq \lambda$ e pelo fato 3.1.5, $j \in O^{k} \cup C^{k}$.

Caracterizamos assim, os elementos de $O^{k} \cup C^{k}$, como aqueles para os quais e possível construir uma sequência de nós adjacentes, $j, a^{k}(j), a_{2}^{k}(j), \ldots, a_{r}^{k}(j)$, ou seja, um passeio entre $a_{r}^{k}(j)$ e $j$, inteiramente contido em $O^{k} \cup C^{k}$.Para r suficientemente grande, podemos detectar passeios ,e até mesmo caminhos, entre a origem e os nós .

Com relação aos rótulos $g^{k}($.$) , temos :$

LEMA 3.1.10

$\operatorname{Sejam}\left(k_{1}, k_{2}\right) \in \mathbb{N} x \mathbb{N k}_{1}<\mathbf{k}_{2}$ ej $\in \mathbb{N}$. 
Se $g^{k_{1}}(j) \neq g^{k_{2}}$ então $g^{k_{1}}(j)>g^{k_{3}}$

\section{Demostração}

Seja k' a primeira iteração do algoritmo para a qual algoritmo temos que

$g^{k_{1}}(j) \neq g^{k^{\prime}}(j), k^{\prime}>k 1$ Do processo de rotulação utilizado pelo $j_{k} \in N$

$g^{k_{1}}(j)=g^{k^{\prime}-1}(j)>g^{k^{\prime}-1}\left(j_{k}\right)+c\left(j_{k}, j\right)==g^{k^{\prime}}\left(j_{k}\right)$ para algum

e segue a afirmação.

Como a aplicação sucessiva de $a^{k}($.) corresponde a "pesquisarmos" algum passeio na orientação oposta a dos arcos que o compö́em, é natural que os valores $g^{k}(j)$ estejam associados ao custos desses passeios.

\section{LEMA 3.1.11}

$\operatorname{Sejam}(k, r) \in \mathbb{N} x \mathbb{N}, j \in O^{\mathbf{k}} \cup C^{\mathbf{k}}$.

Então, $g^{k}(j)=g^{k}(j)+c\left(P_{j}\right)$, onde $\vec{j}=a_{r}^{k}(j)$ e $P_{j}$ o passeio $\left.P_{j}=\left(a_{\bar{z}}^{k}(j), a_{\bar{z}-1}^{k}(j), \ldots, a_{(j)}^{k} j\right), j\right)$, para algum $\bar{s} \leqq r$.

\section{Demonstração}

Do processo construtivo do algoritmo temos que se $j_{2} \in N \backslash\{1\}$ e $a^{k}\left(j_{2}\right)=j_{1}\left(j_{1} \in N\right)$, então $g^{k}\left(j_{2}\right)=g^{k}\left(j_{1}\right)+c\left(j_{1}, j_{2}\right)$. Portanto, $\forall j \in$ $\left(C^{k} \cup O^{k}\right) \backslash\{1\}, g^{k}(j)=g^{k}\left(j_{1}\right)+c\left(P_{1}\right)$, onde $P_{s}=\left(a_{s}^{k}(j), a_{s-1}^{k}(j), \ldots, j\right)$ $\forall s \in \mathbb{N} \mid a_{s-1}^{k}(j) \neq 1$.

Assim, $\forall r \in \mathbb{N}$, tomando-se $\overline{8}, 0$ maior índice

$\bar{s} \leqq r$ tal que $a_{\bar{s}-1}^{k}(j) \neq 1$, segue a afirmação.

Segue dai que : 


\section{LEMA 3.1.12}

Sejam $k \in \mathbb{N}, \mathrm{j} \in O^{\mathrm{k}} \cup \mathrm{C}^{\mathrm{k}} \mid \mathrm{g}^{\mathrm{k}}(\mathrm{j})=\mathrm{g}^{\mathrm{k}+1}(\mathrm{j})$

Então $\forall r \in \mathbb{N} \quad a_{r}^{k}(j)=a_{r}^{k+1}(j)$ e $g^{k}\left(a_{r-1}^{k}(j)\right)=g^{k+1}\left(a_{r-1}^{k+1}(j)\right)$

Demonstração

Do fato 3.1.6, temos que $g^{k}(j)=g^{k+1}(j) \Rightarrow a^{k}(j)=a^{k+1}(j)$. Assim, pelo resultado anterior, segue a afirmação.

Os passeios obtidos a partir da aplicação recursiva de $a^{k}($.$) , cor-$ respondem a caminhos, devido ao fato de estarmos considerando apenas grafos que näo possuam circuitos de custos negativo :

\section{TEOREMA 3.1.13}

Seja $k \in \mathbb{N}, j \in O^{k} \cup C^{k}$.Então existe um caminho $P$ entre a origem e o nó $\mathbf{j}, c(P)=g^{k}(j)$, que pode ser obtido a partir da aplicação recursiva de $a^{k}($.$) , isto é, P=\left(a_{s}^{k}(j), \ldots, a^{k}(j), j\right)$, para algum $r \in \mathbb{N}$.

Demonstração

Por indução sobre o número de iterações

Base de indução

$\mathbf{k}=0$ Imediato

$\mathrm{k}=1$

Neste caso $C^{1}=\{1\}$ e $O^{1}=\operatorname{suc}(1)$ Portanto:

Se $\mathrm{j}=1$ tomamos $P=(1)=\left(a_{0}^{1}(1)\right) \operatorname{com} g^{1}(1)=0=c(P)$

Se $j \in O^{1} P=(1, j)=\left(a^{1}(j), j\right)$, e claramente $g^{1}(j)=c(1, j)=$ $c(P)(j \neq 1$ pois grafo não possue laços).

\section{Hipótese de indução}

Suponhamos que a afirmação seja verdadeira $\forall k 1 \leq k \leq s-1, \forall j \in$ $O^{k} \cup C^{k} \exists P^{k}$, um caminho entre a origem e o nó j com $c\left(P^{k}\right)=g^{k}(j)$ e $P^{k}=\left(a_{r}^{k}(j), \ldots, a^{k}(j), j\right)$ para algum $r_{k} \in \mathbb{N}$. 
$k=8$

$\operatorname{Seja} \mathrm{j} \in O^{k} \cup C^{k}$

i.) $g^{k}(j)=g^{k-1}(j)$

Da hipótese de indução, $\exists P^{k-1}$, um caminho entre $1 \mathrm{ej}, P^{k-1}=$ $\left(a_{r}^{k-1}(j), \ldots, a^{k-1}(j), j\right)$ para algum $\mathrm{r} \in \mathbb{N}, \operatorname{com} g^{k-1}(j)=c\left(P^{k-1}\right)$. Ora, como $g^{k}(j)=g^{k-1}(j)$ vem, do lema 3.1.2 que os rótulos $a_{s}^{k-1}(j)$ e $g^{k-1}\left(a_{s}^{k-1}(j)\right)$ não foram alterados e portanto $P^{k}=\left(a_{r}^{k}(j), \ldots, a^{k}(j), j\right)$ e um caminho entre $1 \mathrm{e} \mathrm{j}, g^{k}(j)=c\left(P^{k}\right)$.

ii.) $g^{k}(j) \neq g^{k-1}(j)$

Nesse caso, $a^{k}(j)=j_{k}\left(j_{k}\right.$ o nó escolhido para fechamento na iteração k). Da hipótese de indução, existe um caminho entre $1 \mathrm{e} j_{k}$, $P^{k-1}=\left(a_{r}^{k}(j), \ldots, a^{k}(j), j\right)$, com $c\left(P^{k-1}\right)=g^{k-1}\left(j_{k}\right)$,

E imediato que $P^{k}=\left(a_{r}^{k}\left(j_{k}\right), \ldots, a^{k}\left(j_{k}\right), j_{k}, j\right)$ e um passeio entre 1 e j de custo $c\left(P^{k}\right)=g^{k}(j)+c(j k, j)=g^{k}(j)$.Como $P^{k-1}$ e um caminho, e suficiente mostrar que j não é um nó de $P^{k-1}$

Suponhamos, por contradição, que

$\left\{s \in \mathbb{N} \mid \mathrm{a}_{\mathrm{B}}^{\mathrm{k}}\left(\mathrm{j}_{\mathrm{k}}\right)=\mathrm{j}, \mathrm{s} \leqq \mathrm{r}\right\} \neq 0$ Tomando-se $\bar{s}$ o menor destes elementos, temos que

$$
\begin{aligned}
& g^{k}(j)=c\left(j_{k}, j\right)+g^{k}\left(j_{k}\right)= \\
& \quad c\left(j_{k}, j\right)+g^{k-1}\left(j_{k}\right)= \\
& \quad c\left(j_{k}, j\right)+c(\hat{P})+g^{k-1}(j) \\
& \text { onde, } P=\left(a_{\bar{g}-1}^{k-1}\left(j_{k}\right), \ldots, a^{k-1}\left(j_{k}\right), j_{k}, j\right)
\end{aligned}
$$

Como $g^{k}(j)<g^{k-1}(j)$ temos $c(\hat{P})+c\left(j_{k}, j\right)<0$ e portanto $\left(a_{\overline{8}-1}^{k-1}\left(j_{k}\right), \ldots, a^{k-1}\left(j_{k}\right), j_{k}, j\right)=\left(j, a_{\bar{g}-1}^{k-1}\left(j_{k}\right), \ldots, a^{k-1}\left(j_{k}\right), j_{k}, j\right)$ é circuito de custo negativo, contrariando a hipótese 3.1.1 .

Logo,$P^{k}$ não possue circuitos, sendo entẫo um caminho. Do princípio de indução segue a tese.

Apesar de termos garantido assim a recuperação de um caminho através dos apontadores $a^{k}($.$) , precisamos verificar que o caminho mínimo$ pode ser determinado. 
Seja $k \in \mathbb{N} \mathbf{j} \in \mathrm{O}^{\mathrm{k}} \cup \mathrm{C}^{\mathrm{k}} \operatorname{Se} g^{k}(j)=g(j),(\mathrm{g}(\mathrm{j})$ o custo do caminho mínimo entre 1 e j), então $\forall k^{\prime} \geqq k g^{k^{f}}(j)=g(j)$.

\section{Demonstração}

Do teorema anterior, $\forall l \in \mathbb{N}, \mathrm{g}^{1}(\mathbf{j}) \geqq \mathrm{g}(\mathrm{j})$. Se $\exists k^{\prime} \in \mathbb{N k}^{\prime} \geqq \mathrm{k} \mid \mathrm{g}^{\mathbf{k}^{\prime}}(\mathbf{j}) \neq$ $\mathrm{g}(\mathrm{j})=\mathrm{g}^{\mathrm{k}}(\mathrm{j})$, então $g^{k^{\prime}}(j)<\mathrm{g}^{k}(j)$ e portanto $g(j) \leqq g^{k^{\prime}}(j)<g(j)$

Absurdo.

Assim tendo determinado o custo de um caminho mínimo entre a origem e um dado nó , o rótulo deste nó não e mais alterado, e portanto o algoritmo A detecta "ótimalidade" de caminhos .Mais ainda temos :

\section{LEMA 3.1.15}

Seja $k^{*} \in \mathbb{N}$.

Se $\mathrm{j} \in C^{k^{*}}$ e $g^{k^{*}}(j)=g(j)$, então $\forall k \geqq k^{*} \quad j \in C^{k^{*}}$

\section{Demonstração}

Trivial pois como $g^{k^{*}}(j)=g(j)$ temos pelo resultado anterior que $\forall k \geq k^{*} g^{k}(j)=g(j)$.Para que o nó fosse reaberto, seria necessário que $g^{k}(j)<g^{k^{*}}(j)$, e segue a afirmação.

Nada impede que um nó fechado venha a ser reaberto, ainda que o algoritmo efetue apenas um número finito de fechamentos e reaberturas ate detectar um caminho de custo mínimo entre 1 e p (caso exista).Contrariamente ao caso de Dijkstra, não é possivel assegurar que ao fechar o nó alvo tenhamos determinado o valor ótimo do problema e portanto não podemos simplesmente tomar como critério de parada do algoritmo, $p \in C^{k}$. Ainda assim, podemos assegurar que para qualquer nó $j$, 0 algoritmo esta sempre pesquisando algum caminho mínimo entre a origem e nó j. 


\section{PROPOSIÇÃO 3.1.16}

Seja $\mathrm{j} \in N$, um nó atingível a partir de 1 .

Então $\forall k \in \mathbb{N}$, ou $g^{k}(j)=g(j)$ ou $\exists i \in O^{k}$ um nó pertencente a algum caminho mínimo entre 1 e j tal que $g^{k}(i)=g(i)$.

\section{Demonstração}

Seja $k \in \mathbb{N}$

Se $g^{k}(j)=g(j)$ nada a fazer.

Caso contrário, seja $P^{*}=\left(j_{1}, j_{2}, \ldots, j_{r}\right)$, um caminho mínimo entre $j_{1}=1$ e $j_{r}=j\left(P^{*}\right.$ existe pois jé atingível a partir de 1 e o grafo não possue circuitos de custo negativo).

Tome $s^{*}$ o maior indice, tal que $\forall s \in\left\{1,2, \ldots, s^{*}\right\}, g^{k}\left(j_{s}\right)=$ $g\left(j_{s}\right), j_{s} \in C^{k}$.Claramente $s^{*}$ existe pois $1 \in C^{k}$ e $g^{k}(1)=g(1)=0$.

Tomemos entäo $\bar{i}=i_{\varepsilon^{*}+1}$

a.) $i \in O^{k}$

$\mathrm{Na}$ iteração $k_{1}$, na qual o nó $i_{g^{*}}$ for fechado, com rótulo $g^{k_{1}}\left(i_{g^{*}}\right)=$ $g\left(i_{s^{*}}\right)$, tinhamos $g^{k_{1}}\left(i_{s^{*}}+1\right)=g^{k_{1}}\left(i_{s^{*}}\right)+c\left(i_{s^{*}}, i_{s^{*}+1}\right)$ e como $P^{*}$ é caminho mínimo, $g^{k_{1}}\left(i_{s^{*}}\right)+c\left(i_{s^{*}}, i_{s^{*}}+1\right)=g\left(i_{z^{*}}+1\right)$ ado.

Logo $g^{k_{1}}\left(i_{s^{*}}+1\right)=g\left(i_{s^{*}+1}\right)$ e portanto esse rótulo não e mais alter-

b.) $\vec{i} \notin O^{k} \mathrm{Na}$ iteração $k_{1}$, na qual o nó $i_{g^{*}}$ for fechado, $i_{s^{*}+1}$ possue rótulo $g^{k_{1}}\left(i_{s^{*}+1}\right)=g\left(i_{s^{*}+1}\right)$, o qual não é mais alterado (observe que $-i$ esta aberto nesta iteração).Assim, se para algum $\left.\hat{k} \geqq k_{1} i_{a^{*}+1}\right) \in C^{\hat{k}}$, então na iteração $k, i_{s^{*}+1} \in C^{k}$ e $g^{k}\left(i_{s^{*}}+1\right)=g\left(i_{z^{*}}+1\right)$, contrariando a escolha de $s^{*}$.Portanto $i \notin C^{k}$.

De a.) e b.) segue a afirmação

Com isso, dentro do critério de parada adotado,o algoritmo não termina enquanto näo obtiver, para cada nó atingível a partir da origem,o custo do caminho mínimo para atingi-lo.Assim, ao terminar, o algoritmo tera detectado, caso exista, uma solução ótima do problema. 


\section{TEOREMA 3.1.17}

Seja $\mathrm{G} \equiv(\mathrm{N}, \mathrm{E})$ um grafo orientado satisfazendo as hipóteses 1.1.2 , 3.1.1 , e a HIPÓTESE BÁSICA.Então, o algoritmo A, quando aplicado a $G$, termina em um número finito de passos, quando um caminho entre 1 e cada nó atingível pode ser obtido através dos rótulos $a^{k}($. $)$.

\section{Demonstração}

A cada iteração, $\exists \mathbf{j} \in O^{k}$ que é fechado pelo algoritmo na iteração k, o qual só será reaberto ao se detectar um caminho de custo estritamente inferior a $g^{k}(j)$.Como $\mathrm{G}$ é finito, o número de caminhos entre $1 \mathrm{e} j$ também o é portanto o processo de reabertura de nós só pode ser executado um número finito de vezes.Então, ao final de um número finito $k^{*}$ de passos, $g^{k^{*}}(j)=g(j)$ e $j \in C^{k^{*}}$, não sendo mais reaberto.Segue que após um número finito de passos, $O^{k}=\emptyset$ e o algoritmo termina.

Mas da proposição anterior,isso só ocorre quando, para cada nó j atingível a partir da origem, $\mathrm{j} \in C^{k}, g^{k}(j)=g(j)$, sendo que um caminho mínimo entre 1 e j pode ser obtido a partir da aplicação sucessiva de $a^{k}($.$) .$

Com isso mostramos a validade do algoritmo $A$ no caso de grafos finitos. Para o caso em que $\mathrm{G}$ e apenas localmente finito, pode-se mostrar que o algoritmo termina nos caso em que apenas um número finito de nós, em particular o nó alvo, pode ser fechado, o que pode ser conseguido através de hipóteses não muito restritivas.*

É importante observar que no processo de demonstração dos resultados, utilizou-se apenas os estmadores $g^{k}($.$) , correspondendo ao custo$ de um caminho entre a origem e os nós, sem que sequer mencionassemos as funções $h^{k}(.) . N a$ verdade, o conjunto de resultados vistos, se aplica a uma classe mais ampla de algoritmos, conhecidos como algoritmos de rotulação, os quais diferem em função do critério de escolha para fechamento

*

H1: Dado $\mathrm{n} \in N, \exists k_{n}>0: \hat{h}(n)<k_{n}$, qualquer iteração H2: $\exists N^{\prime} \subset N, N^{\prime}$ finito $\mid \forall n \in N \backslash N^{\prime} g(n)>h(1)$

H3: $\forall \alpha>0 \exists N^{\prime} \subset N$, finito, tal que

$\forall n \in N \backslash N^{\prime} g(n)+\hat{h}(n)<\infty$ 
utilizado.Podem ser incluidos nessa classe os algoritmos de busca em profundidade, o de busca por espalhamento, onde os elementos menos recentemente gerados são escolhidos, ou mesmo o de busca uniforme, onde $h^{k}()=$.0 . Com relação a este úl timo caso vale:

\section{TEOREMA 3.1.18}

Seja $\mathbf{k} \in \mathbb{N}, \mathbf{k} \geq \mathbf{l j} \in \mathbf{C}^{\mathbf{k}} \mathbf{j}$ e suponhamos que

$c: N \rightarrow \mathbb{R}^{+} \operatorname{eh}^{\mathbf{k}}()=$.0 .

Então $g^{k}(j)=g(j)$

\section{Demonstração}

Por indução sobre o número de iterações

$k=1$

Claramente, $C^{1}=\{1\} \operatorname{eg}^{1}(1)=0=g(1)$

Hipótese de indução

Suponha que a afirmação seja válida $\forall k, 1 \leqq k \leqq s-1$

$k=8$

Seja $\mathrm{j} \in C^{k}$

Se $\mathrm{j} \in C^{k-1}$, da hipótese de indução, $g^{k-1}(j)=g(j)$, e da proposição 3.1.16, $g^{k}(j)=g(j)$.

Se $j \notin C^{k-1}$, entäo $\mathrm{j}$ foi o elemento escolhido para fechamento nessa iteração, isto é, $f^{k-1}(j)=g^{k-1}(j)=\min _{j^{\prime} \in O^{k-1}} g^{k-1}\left(j^{\prime}\right)$

Suponhamos que $g^{k}(j)=g^{k-1}(j)>g(j)$, e sejam

$P_{\min }=\left(i_{1}, i_{2}, \ldots, i_{t}, j\right)$ um caminho mínimo entre $i_{1}=1$ e $j$ e $s^{*}$ - maior índice, $1 \leqq s^{*} \leq t$, para o qual $i_{s^{*}} \in C^{k}$. Claramente, $s^{*}$ existe pois $1 \in C^{1}$ e $g^{1}(1)=g(1)$ e esse nó só seria reaberto com custo inferior, o que não é possível.

Além disso, na iteração em que $s^{*}$ foi fechado, digamos $k^{*}$,

$g^{k^{*}}\left(i_{s^{*}}\right)+c\left(i_{s^{*}}, i_{s^{*}}+1\right)=$

$g\left(i_{a^{*}}\right)+c\left(i_{a^{*}}, i_{s^{*}}+1\right)=$

$g\left(i_{a^{*}}+1\right)$, pois o caminho $P_{\min }$ e mínimo.Assim ,como

$\forall k g^{k}\left(i_{s^{*}}+1\right)=g\left(i_{s^{*}}+1\right)$, temos $g^{k^{*}}\left(i_{8^{*}}+1\right)=g\left(i_{8^{*}}+1\right)$, e portanto 
$\forall k^{\prime} \geqq k^{*} g^{k^{\prime}}\left(i_{s^{*}+1}\right) g\left(i_{s^{*}+1}\right)$, em particular na iteração $\mathbf{k}-1$

Logo, $g(j) i g^{k-1}(j)=g^{k}(j)=\min _{j^{\prime} \in O^{k-1}} g^{k-1}\left(j^{\prime}\right)$ e como $i_{8^{*}+1} \in$ $O^{k}$ por escolha de $s^{*}, g(j)<g^{k-1}\left(i_{s^{*}+1}\right)=g\left(i_{s^{*}+1}\right)$.

Mas por hipótese os custos do grafo são não negativos e portanto $g(j) \geqq g\left(i_{s^{*}}+1\right)$ Assim, $g(j)<g\left(i_{s^{*}+1} \leqq g(j)\right.$.Absurdo.

Logo, se $g^{k}(j)>g(j)$, esse elemento não será escolhido para fechamento,

e portanto $g^{k}(j)=g(j)$.

Do princípio de indução segue a tese.

Este resultado nos mostra que o algoritmo de busca uniforme, isto é, $\operatorname{com} h^{k}()=$.0 , coincide com Dijkstra desde que os custos do grafo sejam não negativos, pois ao fecharmos um nó, teremos detectado um caminho entre a origem e ele, de tal forma que 0 algoritmo não executa o procedimento de reabertura de nos.Com isso, todos os resultados válidos para o algoritmo A se aplicam a Dijkstra, inclusive a determinação de um caminho mínimo entre a origem e cada nó atingível a partir dela.

Um aspecto importante que fica evidenciado com isso e que as funçōes $\left\{h^{h}().\right\}$, juntamente com os custos associados ao grafo, são o fator determinante para o bom desempenho dos algoritmos, $\mathrm{e}$ isso nos induz a um estudo mais detalhado do comportamento destas funções.

\section{2 - CONCLUSÃO}

Vimos então um algoritmo para a resolução do problema do caminho mais curto, que se aplica a problemas onde os custos associados ao grafo podem ser negativos, e que, estruturalmente, mantem uma relação bastante forte com os algoritmos de Dijkstra e Ford.

$\mathrm{O}$ que define o comportamento dos algoritmos são as estimativas utilizadas. Desde que se imponha condições não muito restritivas sobre as mesmas, podemos assegurar um desempenho semelhante ao de Dijkstra, no sentido de que a fechar um no, teremos obtido o custo de um caminho mínimo entre a origem e este nó.

Nosso próximo passo e o estudo do desempenho do algoritmo ao utilizarmos particulares estimativas.Em particular, estudaremos o caso em que as mesmas subestimam a distância entre cada nó e 0 alvo, obtendo então um algoritmo bastante conhecido em inteligência artificial - o algoritmo $A^{*}$. 


\section{Capitulo 4}

\section{0 algoritmo $\mathrm{A}^{*}$ e sua relação com dualidade}

O considerável destaque que o algoritmo $A^{*}$ recebe na literatura, reflete, de certa forma, sua eficiência.Podemos considerá-lo um caso particular do algoritmo $A$, sobre o qual se impöe a restrição de que os estimadores utilizados sejam, na. verdade, subestimadores do caminho mínimo entre cada nó e 0 alvo.

Essa retrição pode reduzir significativamente o número de nós

pesquisados pelo algoritmo $A$ pois pode-se assegurar que, ao fechar - nó alvo, (caso façamos) teremos determinado a soluçäo do problema. Casos particulares de subestimativas permitem, até mesmo, que o algoritmo se comporte como Dijkstra fechando apenas nós para os quais se tenha obtido o custo do caminho mínimo da origem até eles.A determinação de 
"bons" estimadores que induram a um menor número de pesquisas de nós tem sido objeto de estudo de vários autores [Pe83] [Ge78].

Por outro lado o algoritmo $A^{*}$ mantém forte relação com dualidade, ao adotarmos um conceito mais amplo de problema dual, relação esta que permite considerarmos $A^{*}$ um bom algoritmo para a resolução de problemas mais gerais que o problema de determinação de caminhos mínimos.

\section{A Escolha de Estimadores "Inteligentes" - O Algoritmo A}

Vamos admitir neste capítulo a validade de todas as hipóteses assumidas no capitulo precedente. Em particular lembramos que o grafo considerado não possui circuitos de custo negativos, hipótese esta fundamental para nosso trabalho.

Como se pretende utilizar um critério de fechamento de nós que de certa forma induza a uma pesquisa "bem orientada" dos mesmos,é natural que esse critério utilize "boas estimativas" do custo do caminho entre os nós e o alvo.Sendo os estimadores que estamos adotando da forma $f(\cdot)=$ $\hat{g}(\cdot)+(\cdot)$, deve-se verificar quais condiçōes devem ser impostas sobre $h(\cdot)$ para que $f(\cdot)$ seja um bom estimador uma vez que já dispomos de uma maneira de calcular $\hat{g}(\cdot)$,conforme proposto pelo algoritmo $A$.

Naturalmente o "melhor" estimador possível é dado pela própria distância entre os nós e os alvos,isto é, $\hat{h}(j)=k(j, p) \forall j \in N$,porém em termos operacionais sua determinação é tão complexa quanto a resolução do próprio problema. Assim é usual trabalharmos com estimadores menos . fortes.

\section{DEFINIÇĀO 4.1.1}

Uma função $\hat{h}: N \rightarrow \mathbb{R}$

é chamada uma heuristica admissível, ou subestimador admissível,

$$
\forall j \in N \hat{h}(j) \leqq h(j)=k(j, p)
$$


Capítulo f: $O$ algoritmo $\mathbb{A}^{*}$ e sua relação com dualidade

Como os grafos com os quais estamos trabalhando não possuem circuitos de custos negativo, para o nó alvo, temos que $h(p)=k(p, p)=0$ e não é portanto muito restritivo assumirmos a seguinte hipótese :

\section{HIPÓTESE 41.2}

Os estimadores $\left\{h^{k}(.)\right\}_{k \in \mathbb{N}}$ utilizados pelo algoritmo A satisfarem

$$
h^{k}(p)=0 \forall k \in \mathbb{N}
$$

A principal caracteristica de estimadores admissiveis é que,ao utilizálos,o algoritmo A passa a ter um melhor desempenho quanto ao número de aberturas e fechamentos efetuado.

\section{PROPOSIçÃO 41.3}

Se 0 algoritmo A utiliza uma sequência $\left\{h^{k}(\cdot)\right\}_{k \in \mathbb{N}}$ de estimadores admissíveis satisfazendo a hipótese 4.1.2, então, na iteraçäo

$k^{*} \in \mathbb{N}$ na qual o nó p e fechado tem-se

$$
g^{*}(p)=g(p)
$$

\section{Demonstração}

Ao fechar o nó p,tem-se $g^{k^{*}}(p)=c(P)$ para algum caminho $\mathrm{P}$ entre 1 e p,e portanto $g^{k^{*}}(p) \geqq g(p)$.

Se $g^{k^{*}}(p)>g(p)$ temos que $\exists i \in \mathbb{N}$ um nó de algum caminho minimo entre 1 e $p$ tal que $g^{k^{*}}(i)=g(i), i \in O^{k^{*}}$. estimadores.

Assim, $f^{k^{*}}(i)=g(i)+h^{k^{*}}(i) \leqq g(i)+h(i)$ adimissibilidade dos

Portanto,como i é um nó de um caminho minimo, $f^{k^{*}}(i) \leqq g(i)+$ $k(i, p)=g(p)$. 
Capítulo 4: $O$ algoritmo $A^{*}$ e sua relação com dualidade

Ora,mas $h^{k^{*}}(p)=0$ e dai

$f^{k^{*}}(i)=g(p)=g(p)+h^{k^{*}}(p)<g^{k^{*}}(p)+h^{k^{*}}(p)=f^{k^{*}}(p)$

ou seja, $f_{i}^{k^{*}}(i)<f^{k^{*}}(\mathrm{p})$

contrariando a escolha de $p$ para fechamento

Caso o nó p seja atingível a partir de 1 ,esse resultado define um novo critério de parada para o algoritmo A,qual seja, a inclusäo do nó alvo no conjunto de nós fechados. Na verdade trabalho de Nilsson [Ni82] o critério de término do algoritmo $\mathbb{A}$ é dado por $\mathrm{p} \in C^{k}$,e caso $O^{k}=\emptyset$ o algoritmo terminadetectando inviabilidade. Assim, caso o processo termine com $p \in C^{k}$ não se pode assegurar a obtenção do valor ótimo do problema a menos que trabalhemos com estimadores admissiveis.Nilsson chama de $A^{*}$ a a algoritmo A sob a hipotese admissibilidade e,da proposição anterior,ao fechar o nó p o processo detecta o valor ótimo do problema.Assim admitiremos a, seguinte definição :

\section{DEFINIÇÄO 4.1.4}

Chamamos de $A^{*}$ ao algoritmo $\mathbb{A}$ que se utiliza de uma sequência de subestimadores $\left\{h^{k}(\cdot)\right\}_{k \in \mathbb{N}}$ admissiveis satisfazendo a hipótese $4.1 .2 \mathrm{e}$ com critério de parada $\left(O^{k} \neq \emptyset\right.$ e $\left.p \notin c^{k}\right)$ em substituiçäo a $\left(O^{k} \neq \emptyset\right)$.

\section{TEOREMA 4.1.5}

Nas condicōes do teorema 3.1.17 (finitude do algoritmo $A^{*}$ ) 0 algoritmo termina ao final de um número finito de passos,quando então ou detecta inviabilidade do problema, ou um caminho mínimo entre 1 e p pode ser obtido através da aplicação sucessiva de $a^{k}(\cdot)$.

Demonstração: 
Da mesma forma que o teorema 3.1.17, tem- se que ao final de um número finito $k$,de passos ou $\mathrm{p} \in C^{k}$, ou $O^{k}=\emptyset$. Caso $O^{k}=\emptyset \mathrm{e}$ $\mathrm{p} \notin C^{k}, 0$ algoritmo detectou inviabilidade do problema,pela proposição 3.1.16. Caso contrário,da proposição anterior,ao ser fechado $g^{k}(p)=g(p)$ algoritmo termina com a obtenção de $\mathrm{g}(\mathrm{p})$.

A verificação de que o caminho pode se recuperado segue analogamente ao caso do algoritmo $\mathrm{A}$ uma vez que os demais resultados não são invalidados pelo novo critério de parada.

Passemos agora a estudar o desempenho de $A^{*}$.Como a complexidade do algoritmo esta fortemente relacionada com o número de fechamentos e aberturas efetuadas pelo mesmo primeiro passo será verificar as condiçöes necessárias e suficientes para fechamento e / ou reabertura de nós.

0 primeiro resultado nessa linha é o seguinte :

\section{LEMA 4.1.6}

Seja $k \in \mathbb{N}$ uma iteração do algoritmo $A^{*}$ tal que $O^{k} \neq \emptyset$ e $g^{k}(p) \neq$ $g(p)$. Entäo

$$
\exists i \in O^{k} \mid f^{k}(i) \leqq g(p)
$$

\section{Demonstração}

Se $g(p)=+\infty$ nada a fager.

Caso contrário,seja $i \in O^{k} \mid g^{k}(i)=g(i)$,onde $i \in \mathbb{N}$ é um nó de algum caminho mínimo entre 1 e p.

Temos então

$f^{k}(i)=g^{k}(i)+h^{k}(i)=g(i)+h^{k}(i)$
um de um caminho minimo entre 1 e p)

$f^{k}(i)=g^{k}(i)+h^{k}(i)=g(i)+h^{k}(i) \leqq g(i)+h(i)=g(p)$ (pois i é

Esse resultado nos mostra que enquanto 0 algoritmo não terminar,existe uma estimativa de custo de caminho inferior ao valor b́timo do 
Capítulo 1: $O$ algoritmo $A^{*}$ e sua relação com dualidade

problema.Além disso podemos garantir que $A^{*}$ não nós cuja a estimativa de custos de caminho supere $g(p)$.

\section{LEMA 4.1.7}

$A^{*}$.

Seja $j_{k} \in N$ o nó escolhido para fechamento na k-ésima iteração de

Então $f^{k}\left(j_{k}\right) \leqq g(p)$

Demonstração

Se $g(p)=+\infty$ nada a fazer.

Caso contrário, na iteração $\mathrm{k}, \exists i \in O^{k} \mid f^{k}(i) \leqq g(p)$ e portanto do critério de escollha para fechamento,

$$
f^{k}\left(j_{k}\right) \leqq f^{k}(i) \leqq g(p)
$$

Ou seja,mostramos que o algoritmo fecha apenas os nós que podem fager parte de algum caminho minimo entre a origem e 0 alvo.A condição $f^{k}(j) \leqq g(p)$ não é entretanto uma condição suficiente para que o nó j seja fechado conforme o seguinte exemplo:

\section{EXEMPLO 4.1.8}

Considere o grafo $G=(N, E)$

$N=\{1,2,3\}$

$E=\left\{e_{1}, e_{2}, e_{3}\right\}$

$c\left(e_{1}\right)=c\left(e_{2}\right)=5 \quad c\left(e_{3}\right)=7$

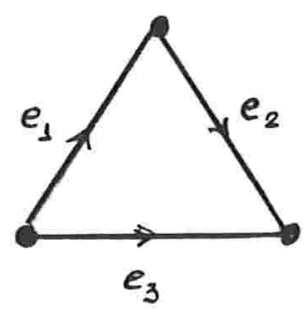

e suponhamos que as estimativas utilizadas sejam constantes, $\hat{h}(1)=7, \hat{h}(2)=2, \hat{h}(3)=0$ (claramente admissiveis). A sequência de conjuntos. ada por $A^{*}$ é dada por 
Capítulo 4: $O$ algoritmo $A^{*}$ e sua relação com dualidade

$$
\begin{aligned}
& O^{0}=\{1\} C^{0}=\emptyset, f^{0}(1)=7 f^{0}(2)=f^{0}(3)=+\infty \\
& O^{1}=\{2,3\} C^{1}=\{1\}, f^{1}(1)=7 f^{1}(2)=7 f^{1}(3)=7
\end{aligned}
$$

Tomando-se para fechamento o nó 3 temos

$$
O^{2}=\{2\} C^{2}=\{1,3\} f^{2}(1)=7 f^{2}(2)=7^{2}(3)=7
$$

e $o$ algoritmo termina,sem fechar o nó $2,\left(f^{2}(2)=g(3)\right)$.

Observe que no exemplo acima o nó 2 poderia ter sido fechado,o que dependia exclusivamente do critério de escolha para fechamento utilizado.Na verdade o que impede que se afirme que um nó satisfazendo $f^{k}(j) \leqq g(p)$ seja fechado é que,nos casos em que ocorre igualdade não dispomos de um critério assegure a escolha de $\mathrm{j}$ fechamento,podendo o nó $p \in N$ ser fechado antes de $\mathrm{j}$.

Vale no entanto

\section{LEMA 4.1.9}

Seja $k^{*} \in \mathbb{N}$ tal que $\mathrm{j} \in \mathrm{O}^{k^{*}}$

Se $\forall k \geqq \cdot k^{*} f^{k}(j)<g(p)$ então $\exists k \in N \mid j \in C^{k}$

Demonstração

Se $g(p)=+\infty$ então o algoritmo termina com $O^{k}=\emptyset$ e a afirmação segue trivialmente.

Se $g(p) \in \mathbb{R}$ então $\forall k \geq k^{*}$,

$f^{k}(j)<g(p)=g(p)+\bar{h}^{k}(p) \leqq g^{k}(p)+h^{k}(p)=f^{k}(p) e$

portanto como $\mathrm{j} \in \mathrm{O}^{k^{*}}$ o nó álvo não é escolhido para fechamento antes que $\mathrm{j} 0$ seja.

Um corolário imediato deste resultado é o seguinte : 
Capítulo 4: $O$ algoritmo $A^{*}$ e sua relaşão com dualidade

\section{COROLÁRIO 4.1.10}

Suponhamos que a sequência de estimadores utilizados por $A^{*}$ seja invariante com as iteracões,isto é, $h^{k}(\cdot)=\hat{h}(\cdot) \forall k \in \mathbb{N}$.

Se $\exists k^{*} \in N f^{k^{*}}(j)<g(p), j \in O^{k^{*}}$ então

$$
\exists \bar{k} \in \mathbb{N} \mid \mathbf{j} \in \mathbb{C}^{\mathbb{E}}
$$

\section{Demonstração}

Trivial observando que a sequência $\left\{g^{k}(j)\right\}_{k \in \mathbb{N}}$ é não decrescente e portanto se $\exists k^{*} \in \mathbb{N} / \mathrm{f}^{\mathbf{k}^{*}}(\mathrm{j})<\mathrm{g}(\mathrm{p})$ então $\forall k \geqq k^{*} f^{k}(j)<g(p)$.

Logo estamos nas condicöes do lema anterior e segue a afirmação.

Ainda que valham estes resultados não podemos garantir que em dado nó $j$ seja fechado antes que $p$ o seja pois nada assegura que $j$ seja aberto antes de $p$ ter sido fechado. Esse é o caso do seguinte exemplo

\section{EXEMPLO 4.1.11}

Seja $G=(N, E)$ dado por

$N=\{1,2,3,4\}$

$E=\{(1,4),(1,2),(2,3),(3,4)\}$

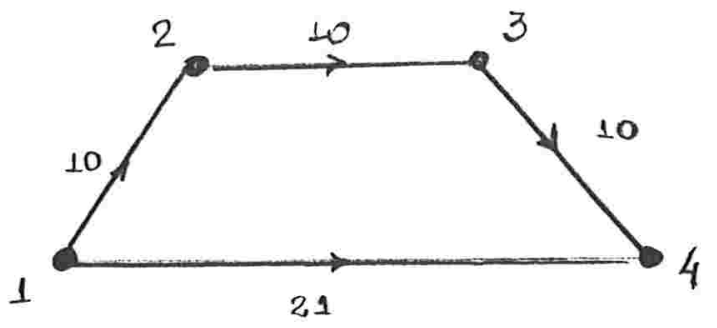

e suponha que $\left\{h^{k}(\cdot)\right\}_{k \in \mathbb{N}}$ seja invariante com as iteracões

$$
h^{k}(1)=21 h^{k}(2)=20 h^{k}(3)=0 \quad h^{k}(4)=0
$$

É fácil verificar que a sequência de nós fechados por $A^{*}$ nesse caso é $n_{1}=1 n_{2}=4$ e o algoritmo termina.

Observe que caso o nó 3 tivesse sido aberto $f^{k}(3)=20<f^{k}(1)$ 
Capítulo f: $O$ algoritmo $A^{*}$ e sua relação com dualidade

Para que possamos garantir o fechamento de um nó é preciso trabalhar com uma condição mais forte.

\section{DEFINIÇÃO 4.1.12}

Dizemos que um caminho $P=\left(i_{1}, i_{2}, \ldots \ldots . i_{r}\right)$ entre $i_{1} \in N$ e $i_{r} \in N$ é (estritamente) C-limitado se

$$
\forall j \in\{1,2, \ldots, r\} f\left(i_{j}\right)+g_{P}\left(i_{j}\right)+\hat{h}\left(i_{j}\right) \leqq C\left(f\left(i_{j}\right)<c\right)
$$

onde $g_{P}\left(i_{j}\right)$ denota o custo do caminho $P_{i j}=\left(i_{1}, i_{2}, \ldots . . i_{j}\right)$ e $\hat{h}\left(i_{j}\right)$ é um subestimador de $k\left(i_{j}, p\right)$.

Quando necessário diremos que um caminho é C-limitado em $\hat{h}(\cdot)$ para definir o estimador utilizado.

Ou seja, um caminho $\mathrm{P}$ e C-limitado quando para cada nó n do mesmo a estimativa de custo de um cminho entre $i_{1}$ e $i_{r}$, restrito a passar pelo trecho do caminho $P$ entre $i_{1}$ e n,não exceder $C$.

\section{PROPOSIÇÃO 4.1.13}

Suponhamos que a sequência $\left\{h^{k}(\cdot)\right\}_{k \in \mathbb{N}}$ seja tal que

$\forall k \in \mathbb{N} \forall j \in N, f^{k}(j) \gtreqless f^{k+1}(j)$ e considere $\mathrm{j} \in N$ tal que $\mathrm{j} \in C^{k^{*}}$ para alguma iteração $k^{*}$ do algoritmo $A^{*}$.

Então $\forall k \in \mathbb{N} \mathbf{k} \geqq$. $\mathbf{k}^{*}$ existe um caminho $v^{*}$-limitado em $h^{k}($. entre $1 \mathrm{e}$ j,onde $v^{*}=g(p)$.

\section{Demonstração}

Ao ser fechado na iteração $k^{*}, f^{k^{*}}(j) \leqq v^{*}$ do lema 4.1.7.

Considere então o caminho $\mathbf{P}=\left(i_{1}, i_{2}, \ldots, i_{r}\right)$ associado $j$ nessa iteração conforme o teorema 3.1.8. É claro,pelo processo construtivo de caminhos que $\forall j \in\{1,2, \ldots, r-1\}, i_{j}$ foi fechado em alguma iteração $k_{i}$ anterior a 
Capítulo f: $O$ algoritmo $A^{*}$ e sua relaçäo com dualidade

$k^{*}$,quando $f^{k_{i}}(j) \leqq v^{*}$.Mas em todas as iteracões $\mathrm{k}$ subsequentes a de seu fechamento, $f^{k}\left(i_{j} \bar{T}=g^{k}\left(i_{j}\right)+h^{k}\left(i_{j}\right) \leqq g^{k_{i}}\left(i_{j}\right)+h^{k_{i}}\left(i_{j}\right)=f^{k_{i}}\left(i_{j}\right) \leqq v^{*}\right.$ Portanto P e $v^{*}$ limitado em $\bar{h}^{k}(\cdot) \forall k \geqq \cdot k^{*}$

Uma consequência imediata disse resultado é :

\section{COROLÁRIO 4.1.14}

Se a sequência de funções $\left\{h^{k}(.)\right\}_{k \in \mathbb{N}}$ é invariante com as iterações, isto é, $h^{k}(j)=\hat{h}(j) \forall k \in \mathbb{N} \forall \mathbf{j} \in \mathrm{N}$ e para alguma iteração $\mathrm{k}$ do algoritmo $A^{*}, j \in C^{h}$,então existe um caminho $v^{*}$ limitado entre $1 \mathrm{ej}$.

\section{Demonstração}

Trivial,uma vez que como $\left\{h^{k}(.)\right\}_{k \in \mathbb{N}}$ é constante e os valores $\left\{g^{k}(.)\right\}_{k \in \mathbb{N}}$ são não crescentes $\forall j \in N$,a sequência $\left\{f^{k}(j)\right\}_{k \in \mathbb{N}}$ é não crescente e estamos sob as condiçóes do resultado anterior.

Vale uma recíproca desses resultados.

\section{PROPOSICÃO 4.1.15}

Suponhamos que a sequência $\left\{h^{k}(.)\right\}_{k \in \mathbb{N}}$ seja tal que

$$
\forall k \in \mathbb{N} \forall \mathbf{j} \in \mathbb{N}, \mathbf{f}^{\mathbf{k}}(\mathbf{j}) \geqq \mathrm{f}^{\mathbf{k}+1}(\mathbf{j})
$$

e considere $\mathrm{j} \in N$ um nó para o qual exista um caminho estritamente $v^{*}$-limitado $\left(v^{*}=g(p)\right)$ entre $1 \mathrm{e}$ para alguma iteração $k^{*}$ antes do término do algoritmo.

Entäo $\exists k \in \mathbb{N}, \mathbf{k} \geq=\mathbf{k}^{*} \mid \mathbf{j} \in \mathbf{C}^{\mathbf{k}}$.

Demonstração 
Capítulo 4: $O$ algoritmo $A^{*}$ e sua relaşão com dualidade

Suponhamos que exista $P=\left(i_{1}, i_{2}, \ldots, i_{r}\right)$ um caminho entre $i_{1}=1 \mathrm{e}$ $i_{r}=j$ estritamente $v^{*}$-limitado na iteração $k^{*}$ e que o nó $\mathrm{j}$ não seja fechado pelo algoritmo.

Seja $8^{*}$ o maior índice, $1 \leq 8^{*} \leq=-1$ para o qual,

$\forall l \in\left\{1,2, \ldots, 8^{*}\right\} i_{l} \in C^{k}, k$ a iteração na qual o algoritmo termina.Claramente $s^{*}$ existe pois $1 \in C^{k}$ e $j \in C^{k}$. $h^{k}\left(i_{s}+1\right)$.

Temos então $i_{\varepsilon^{*}+1} \in O^{K}$ e além disso $f^{k}\left(i_{\theta^{*}+1}\right) \leqq g^{K}\left(i_{8^{*}}+1\right)+$

Como $\forall k \in \mathbb{N} H \in\left\{1,2, \ldots, \mathrm{s}^{*}\right\} g_{\left(i_{i+1}\right)}^{k} \leqq \cdot g^{k}\left(i_{i}\right)+c\left(i_{i}, i_{l+1}\right)$ e esses rótulos são não crescentes com as iteraçôes,

$\forall k \geqq k^{*} g^{k}\left(i_{l}\right) \leqq g_{p}\left(i_{l}\right)\left(g_{p}\left(i_{l}\right)=c\left(P_{l}\right)=\left(i_{1}, i_{2}, \ldots \ldots, i_{l}\right)\right)$.

fechado.Portanto:

Em particular, $g^{k}\left(i_{z^{*}+1}\right) \leqq g_{p}\left(i_{g^{*}+1}\right)$ iteração na qual $i_{g^{*}}$ foi

$$
\begin{gathered}
f^{k}\left(i_{s^{*}}+1\right)=g^{k}\left(i_{8^{*}+1}\right)+h^{k}\left(i_{8^{*}+1}\right) \leqq \\
g^{k^{*}}\left(i_{\theta^{*}+1}\right)+h^{k^{*}}\left(i_{\theta^{*}+1}\right) \leqq \\
\leqq \\
\leqq
\end{gathered}
$$

(pois $\mathrm{P}$ e estritamente $v^{*}$-limitado para $k^{*}$ ). to e portanto

Como $i_{s^{*}+1} \in O^{k}, 0$ algoritmo termina escolhendo $p$ para fechamen-

$$
f^{\bar{k}}(p) \leqq f^{\bar{h}}\left(i_{z^{*}+1}\right)
$$

Mas $v^{*}=f^{h}(p) \leqq f^{h}\left(i_{s^{*}+1}\right)<v^{*}$ Absurdo.

Logo $\forall l \in\{1,2, \ldots, r\} \exists k \leqq k \mid i_{l} \in C^{k}$

e segue a afirmaçäo

E importante observar que nesse caso a existência do caminho mínimo é suficiente para assegurar que um dado no sera explorado,o que não ocorria no lema 4.1.9.esses conceitos caminhos $v^{*}$-limitados nos permitem estudar o desempenho de $A^{*}$ a utilizarmos diferentes heurísticas. Veja que 
um fator que deve ser consideradoao estudarmos a performance de $A^{*}$ é o número de aberturas e fechamentos efetuado pelo mesmo,e a utilização de tais conceitos permite que asseguremos que um dado nó será explorado.

\section{DEFINICAO 4.1.16}

Dadas duas estimativas

$$
\begin{aligned}
& \hat{h}_{1}: N \rightarrow \mathbb{R} \\
& \hat{h}_{2}: N \rightarrow \mathbb{R} \text { diremos que }
\end{aligned}
$$

$\hat{h}_{2}($.$) é mais informada que \hat{h}_{1}($.$) se$

$$
\forall j \in N \backslash\{p\} \hat{h}_{2}(j)>\hat{h}_{1}(j)
$$

\section{DEFINICAO 4.1.17}

Sejam $A_{1}^{*}$ e $A_{2}^{*}$ os algoritmos $A^{*}$ que se utilizam das sequências de heuristicas $\left\{h_{1}^{k}(.)\right\}_{k \in \mathbb{N}}$ e $\left\{h_{2}^{k}(.)\right\}_{k \in \mathbb{N}}$ respectivamente.

Dizemos que o algoritmo $A_{2}^{*}$ domina $A_{1}^{*}$ se $\forall j \in N$, se j é fechado por $A_{2}^{*}$ em alguma iteração antes do término do algoritmo,então $\mathrm{j}$ também é fechado por $A_{1}^{*}$.

Com essas definiçčes podemos então garantir que heurísticas mais informadas conduzem a um melhor desempenho do algoritmo em termos de número de nós fechados.

\section{TEOREMA 4.1.18}

Sejam $A_{1}^{*}$ e $A_{2}^{*}$ os algoritmos obtidos a partir de $A^{*}$ utilizando-se a sequência dos estimadores $\left\{h_{1}^{k}(.)\right\}_{k \in \mathbb{N}}$ e $\left\{h_{2}^{k}(.)\right\}_{k \in \mathbb{N}}$

Se $\forall k \in \mathbb{N}_{2}^{k}($.$) é mais informada que h_{1}^{k}($.$) então A_{2}^{*}$ domina $A_{1}^{*}$.

Demonstração 
Seja j $\in N$ um nó fechado pelo algoritmo $A_{2}^{*} \mathrm{em}$ alguma iteração $k^{*}$ antes do seu término .Então da proposição 4.1.13 $\forall k \geqq k^{*}$ existe um caminho $v^{*}$-limitado $\quad \mathbf{P}=\left(i_{1}, i_{2}, \ldots . ., i_{r}\right) i_{1} \stackrel{\equiv}{=} 1, \iota_{r}=j$ onde

$\forall l \in\{1,2, \ldots, r\} f^{k}\left(i_{l}\right)=g^{k}\left(i_{i}\right)+h_{2}^{k}\left(i_{i}\right) \leqq v^{*} \forall k \geqq k^{*}$

Assim,

$\forall k \geqq k^{*} g^{k}(i)+h_{1}^{k}(i)<g^{k}(i)+h_{2}^{k}(i) \leqq v^{*}$

e portanto existe um caminho $v^{*}$-limitado entre 1 e j para 0 algoritmo $A_{1}^{\natural}$.Assim da proposição 4.1.15 $\exists k_{1} \in \mathbb{N}$ uma iteração antes do término de $A_{1}^{*}$ para o qual o nó j e fechado.

Logo $A_{2}^{*}$ domina $A_{1}^{*}$.

Intuitivamente,o melhor subestimador possível é o próprio custo do caminho mínimo,que corresponde ao estimador "perfeito". Isso se confirma pelo teorema anterior,pois sendo subestimador mais alto,para qualquer outra estimativa utilizada,o algoritmo fechará tantos nós quanto aqueles fechados por $A^{*}$ ao trabalhar com $\hat{h}(i)=k(i, p)$.

Neste caso,resultados aindamais fortes podem ser garantidos.

\section{PROPOSICAO 4.1.19}

Suponhamos que a sequência de estimadores utilizada por $A^{*}$ seja invariante com as iterações. $h^{k}(i)=k(i, p) \forall i \in N$,suponha que o problema seja viável.

Se 0 algoritmo escolhe para fechamento,nos casos em que há empate,o nó cuja estimativa $h^{k}(j)$ seja mínimo,então $A^{*}$ fecha apenas nós pertencentes a caminhos mínimos.

\section{Demonstração}

Como p é atingivel a partir da origem,ao final de um número finito de passos o algoritmo inclui $p$ no conjunto de fechados $e$ termina.

Considere então o caminho mínimo determinado pelo algoritmo,

$$
P^{k}=\left(i_{1}, i_{2}, \ldots, i_{r}, p\right) i_{1}=1
$$


Suponhamos que $\exists n \in N$ tal que $\mathrm{n} \in C^{k+1} \backslash C^{k}$ para algum $\hat{k} \in N$ e seja $s^{*} \in\{1,2, \ldots, r\}$ tal que $\forall l \in\left\{1,2, \ldots, 8^{*}\right\} i_{l} \in C^{k}, i_{s^{*}+1} \notin C^{k}$

(e portanto $i_{s^{*}+1} \in O^{k}$ )

Temos então

$f^{k}(n) \leqq f^{k}\left(i_{s^{*}+1}\right)=g^{k}\left(i_{s^{*}+1}\right)+h^{h}\left(i_{s^{*}+1}\right)=$

$=g^{k}\left(i_{8^{*}+1}\right)+k\left(i_{8^{*}+1}, p\right)=g\left(i_{8^{*}+1}\right)+k\left(i_{8^{*}+1}, p\right)=$

$=g(p)$

Além disso

$f^{k}(n)=g^{k}(n)+h^{k}(n)=$

$g^{k}(n)+k(n, p) \geqq g(n)+k(n, p) \geqq g(p)$

Logo, $f^{k}(n)=g^{k}(n)+h^{k}(n)=g(p)$

e portanto como $g^{k}(n)$ corresponde ao custo de um caminho entre 1 e n e $h^{k}(n)=k(n, p)$ vem que $g^{k}(n)=g(n)$ e portanto o nó pertence a algum caminho mínimo $P$ entre 1 e $p$.

Nada pode ser afirmado,no caso geral,quanto ao número de caminhos mínimos pesquisados,como mostra o seguinte exemplo:

\section{EXEMPLO 4.1.20}

Seja $\mathrm{G} \equiv(N, E)$ o seguinte grafo

$$
\begin{aligned}
& N=\{1,2,3,4\} \\
& \mathrm{E}=\left\{e_{1}, e_{2}, e_{3}, e_{4}\right\} \\
& c\left(e_{1}\right)=c\left(e_{2}\right)=0 \\
& c\left(e_{3}\right)=c\left(e_{4}\right)=10
\end{aligned}
$$

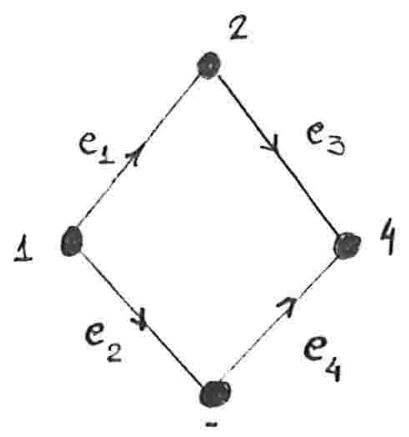

Admitindo-se o criterio de escollha do teorema anterior a sequência de fechamento poderia ser $j_{0}=1, j_{1}=2, j_{2}=3, j_{4}=4$ e teriamos pesquisados todos os caminhos mínimos entre a origem e 0 alvo. 
Apenas em casos particulares,por exemplo,quando os custos associados ao grafo são estritamente positivos,é que se pode assegurar a pesquisa de um único caminho (no sentido de que o algoritmo escolhe para fechameto uma sequência de nós adjacentes).

Sendo a melhor estimativa o custo do caminho mínimo,e natural que tentemos impor condiçôes sobre as heurísticas utilizadas,que assegure um comportamento semelhante 80 de $k(., p)$

Consideremos então a seguinte definição:

\section{DEFINIÇÄO 4.1.21}

Dizemos que um estimador $\hat{h}: N \rightarrow \mathbb{R}$ é consistente se :

$$
\forall(i, j) \in N x N \hat{h}(i) \leqq k(i, j)+\hat{h}(j)
$$

Consistência é uma propriedade bastante semelhante à " propriedade triangular" de $\mathbf{k}(.,$.$) , isto é,$ resultados bastante interessantes sejam obtidos.

$$
\forall(i, j, l) \in N x N x N k(i, j) \leqq k(i, l)+k(l, j) \text { e permite que }
$$

\section{FATO 4.1.22}

\footnotetext{
Seja $\hat{h}: N \rightarrow \overline{\mathbb{R}}$ um estimador consistente. Se $\hat{h}(p)=0$ então $\hat{h}($. é admissível.
}

Demonstração

$\forall j \in N, \hat{h}(j) \leqq k(j, p)+\hat{h}(p)=k(j, p)$

Logo $h($.$) é admissível$

Sob a forma considerada, a verificação de consistência pode ser impraticável Consideremos entảo a seguinte definiçåo : 
Capítulo 4: $O$ algoritmo $A^{*} e$ sua relação com dualidade

\section{DEFINIÇÃO 4.1 .23}

Dizemos que o estimador $\hat{h} N \rightarrow \mathbb{R}$ é uma heurística MONÓTONA se, $\forall(i, j) \in E, \hat{h}(i) \leq c(i, j)+\hat{h}(j)$.

Ou seja, $\hat{h}($.$) é monótona caso se comporte como uma heurístca$ consistente para nós adjacentes do grafo. Naturalmente heurísticas consistentes så̃o também monótonas da própria definiçấo. Entretanto, vale também a reciproca como mostramos a seguir.

\section{LEMA 4.1.24}

Seja $\hat{h} N \rightarrow \overline{\mathbb{R}}$ uma heurística monótona. Então, $\hat{h}($.$) é consistente.$

\section{Demonstração}

Sejam $i \in N$ e $\mathrm{j} \in N$

Se j não é acessível a partir de i, isto é, se não existe um caminho entre i e $j, k(i, j)=-\infty$ e a afirmação segue trivialmente.

Caso contrário,seja $P$ um caminho qualquer entre i $\mathrm{e} j, \mathrm{P} \equiv\left(i_{1}, i_{2}, \ldots, i_{r}\right)$.Send $\hat{h}($.$) monótona por hipótese, \forall k, 1 \leqq k \leqq r-1 \hat{h}\left(i_{k}\right) \leqq \hat{h}\left(i_{k+1}\right)$ e pode se verificar facilmente que:

$$
\begin{aligned}
& \hat{h}\left(i_{1}\right) \leq C_{i_{1} i_{2}}+\hat{h}\left(i_{2}\right) \\
& \leqq c_{i_{1} i_{2}}+c_{i_{3} i_{3}}+\hat{h}\left(i_{3}\right) \leqq \ldots \leqq \sum_{k=1}^{r-1} c_{i_{k} i_{k+1}}+\hat{h}\left(i_{r}\right) \\
& \text { Ou seja, } \hat{h}(i) \leqq c(P)+\hat{h}(j) \text {. }
\end{aligned}
$$

Ora, mas $P$ é um caminho qualquer entre i e j, donde $\hat{h}(i) \leqq k(i, j)+\hat{h}(j)$ e segue a afirmação

É claro que apesar deste resultado reduæir significativamente nosso trabalho em termos da determinação da consistência,a verificação exaustiva. 
da monotocidade de $\hat{h}($.$) pode ser inviável e consequenternente a criação de$ $\widehat{\mathrm{h}}\left(\right.$.) é um aspecto considerável no estudo de $A^{*}$.

Já estudamos dois estimadores consistentes. $O$ primeiro foi visto em Dijkstra;este algoritmo é um caso particular de $A^{*}$ ao utilizarmos a sequência de estimadores invariantes com as iteraçöes, $h^{k}()=$.0 . Esse estimador satisfaz trivialmente $h^{k}(i)=0 \leqq k(i, j)+\hat{h}(j)=k(i, j) \forall(i, j) \in$ $N x N$, pois considera-se apenas grafos com custos näo negativos.

O segundo estimador dado pela própria função distância,isto é, $\hat{h}(j)=k(j, p)$ é também,claramente consistente.

Veja que esses dois casos possuem um ponto,importante, em comum : ambos fecham apenas nós pertencentes a caminhos mínimos entre a origem e os nós.

\section{TEOREMA 4.1.25}

Se os estimadores utilizados por $A^{*}$ são consistentes,isto é $\forall k \in$ $\mathbb{N h}^{\mathrm{k}}($.$) é consistente,então$

$\forall j \in C^{k} g^{k}(j)=g(j)$

\section{Demonstração}

Seja j $\in C^{k}$, k uma iteração qualquer de $A^{*}$.

Na iteração $k^{*}$ na qual o nó jé fechado temos,pela proposição 3.1 .16 que, ou $g^{k^{*}}(j)=g(j)$, ou $\exists i \in 0^{k^{*}} \mid g^{k^{*}}(i)=g(i)$ para i pertencente a algum caminho mínimo entre i e $j, P=\left(i_{1}, i_{2}, \ldots, i_{r}\right)$

Suponhamos que $g^{k^{*}}(j)>g(j)$ Entäo

$$
\begin{gathered}
f^{k^{*}}(i)=g^{k^{*}}(i)+h^{k^{*}}(i)= \\
=g(i)+h^{k^{*}}(i) \leqq \\
\leqq g(i)+h\left(i_{i}, i_{r}\right)+h^{k^{*}}\left(i_{r}\right)=
\end{gathered}
$$


Capítulo f: $O$ algoritmo $A^{*}$ e sua relação com dualidade

$$
\begin{gathered}
=g\left(i_{r}\right)+h^{k^{*}}\left(i_{r}\right)= \\
=g(j)+h^{k^{*}}(j)< \\
<g^{k^{*}}(j)+h^{*}(j)=f^{k^{*}}(j)
\end{gathered}
$$

contrariando a escolha do nó j para fechamento . Assim, $g^{k^{*}}(j)=$ $g(j)$ e portanto esse rótulo não é mais alterado.

Logo,na iteração $\mathbf{k}, g^{k}(\mathrm{j})=\mathrm{g}(\mathrm{j})$

Segue dai que

\section{COROLARIO 4.1.26}

Nas condições do teorema anterior, sendo $\mathrm{j} \in \mathbb{N}$ temos que se $\exists k^{*} \in$ $N \mid j \in C^{k^{*}}$

então $\forall k \geqq k^{*} j \in C^{k^{*}}$.

\section{Demonstração}

Trivial pelo problema anterior e pela lema 3.1.15

Ao trabalharmos com estimadores consistentes teremos assegurado então, um comportamento de $A^{*}$ semelhante ao de Dijkstra,e que naturalmente diminui sua complexidade,conforme estudaremos adiante.Outras propriedades às válidas no caso de Dijkstra podem ser verificadas.

\section{PROPOSIÇÃO 4.1.27}

Seja $\left\{h^{k}(.)\right\}_{k \in N}$ uma sequência de subestimadores consistentes sob a hipótese $4.1 .2\left(h^{k}(p)=0 \forall k \in \mathbb{N}\right)$ 
Capítulo 1: $O$ algoritmo $A^{*}$ e sua relação com dualidade

Se o algoritmo $A^{*}$ utiliza a sequência $\left\{h_{m}^{k}(.)\right\}_{k \in \mathbb{N}}$ definida por $h_{m}^{1}()=.h^{1}($.

$$
h_{m}^{k+1}(j)=\max \left\{h^{k}(j), h^{k+1}(j)\right\} \forall j \in N \forall k \in \mathbb{N}
$$

entäo

$$
\{E(k)\}_{k \in \mathbb{N}}\left(F(k)=f^{k}\left(j^{k}\right)\right)
$$

é năo decrescente.

Demonstração

Primeiramente observe que $\forall k \in \mathbb{N} h_{\mathbf{m}}^{\mathbf{k}}($.$) é consistente pois$

$$
\begin{gathered}
\forall(i, j) \in N x N h_{m}^{k+1}(i) \\
=\max \left\{h^{k}(i), h^{k+1}(i)\right\} \leqq \\
\leqq \max \left\{k(i, j)+h^{k}(j), k(i, j)+h^{k+1}(j)\right\}= \\
=k(i, j)+\max \left\{h^{k}(j), h^{k+1}(j)\right\} \\
=k(i, j)+h_{m}^{k+1}(j)
\end{gathered}
$$

mente.

Sejam $j_{k}$ e $j_{k+1}$ os nÓs fechados nas iterações $k$ e $k+1$ respectiva-

Consideremos dois casos :

i) $g^{k}\left(j_{k+1}\right) \neq g^{k+1}\left(j_{k+1}\right)$

Neste caso

$j_{k+1} \in \operatorname{auc}\left(j_{r}\right)$ e $g^{k+1}\left(j_{k+1}\right)=g^{k}\left(j_{k}\right)+c\left(j_{k}, j_{k+1}\right)$

Assim, $F(k)=f^{h}\left(j_{k}\right)=g^{k}\left(j_{k}\right)+h_{m}^{k}\left(j_{k}\right)$

tanto

Mas por construção, $\forall j \in N\left\{h_{m}^{k}(j)\right\}_{k \in N}$ é näo decrescente e por- 
Capítulo f: $O$ algoritmo $A^{*}$ e sua relaşão com dualidade

$f(k) \leq g^{k}\left(j_{k}\right)+h_{m}^{k+1}\left(j_{k}\right)$

Sendo $h_{m}^{k+1}($.$) consistente, temos$

$$
\begin{gathered}
F(k) \leqq-g^{k}\left(j_{k}\right)+c\left(j_{r}, j_{r+1}\right)+h_{m}^{k+1}\left(j_{r+1}\right) \\
=g^{k+1}\left(j_{r+1}\right)+h_{m}^{k+1}\left(j_{r+1}\right)= \\
=f^{k+1}\left(j_{r+1}\right)=F(k+1)
\end{gathered}
$$

Logo $F(k) \leqq F(k+1)$

ii) $g^{k}\left(j_{r+1}\right)=g^{k+1}\left(j_{r+1}\right)$

Neste caso

$$
\begin{gathered}
F(k)=f^{k}\left(j_{r}\right) \leqq f^{k}\left(j_{r+1}\right) \\
=g^{k}\left(j_{r+1}\right)+h_{m}^{k}\left(j_{r+1}\right)= \\
=g^{k+1}\left(j_{r+1}\right)+h_{m}^{k}\left(j_{r+1}\right) \\
\leqq g^{k+1}\left(j_{r+1}\right)+h_{m}^{k+1}\left(j_{r+1}\right)= \\
=f^{k+1}\left(j_{r+1}\right)=F(k+1)
\end{gathered}
$$

Ou seja, $F(k) \leqq f(k+1)$ crescente.

Sendo a afirmação válida $\forall k \in \mathbb{N}$ segue que $\{f(k)\}_{k \in N}$ é não de-

Como consequência deste resultado seguem os seguintes corolários 
Se a sequência de estimadores utilizadas por $A^{*}$ é não decrescente em $k$, (isto é, $\forall j \in N\left\{h^{k}(j)\right\}_{k \in N}$ é não decrescente) e consistente, então $\{F(k)\}_{k \in \mathbb{N}}$ é não decrescente.

\section{Demonstração}

Trivial, observando que neste caso

$h_{m}^{k}(.) \equiv h^{k}(.) \forall k \in \mathbb{N}$

\section{Além disso,}

\section{COROLÁRIO 4.1.29}

Se a sequência de estimadores utilizada por $A^{*}$ é invariante com as iteraçőes e consistentes,entäo $\{F(k)\}_{k \in \text { IN }}$ é nẳo decrescente.

Novamente se manteve a similaridade entre os resultados válidos para $A^{*}$ sob a hipóotese de consistênciados estimadores e o algoritmo de Dijkstra. $\mathrm{O}$ mais interessante seria se pudessemos assegurar a mesma complexidade que a obtida no algoritmo de Dijkstra,polinomial em relação ao número de nós do grafo.

$\mathrm{DE} A^{*}$

\subsection{ALGUNS COMENTÁRIOS SOBRE A COMPLEXIDADE}

Poderíamos citar alguns trabalhos bastante interessantes quanto a complexidade de $\boldsymbol{A}^{*}$.Martelli [Ma77], baseado em um trabalho anterior de Johnson [Jo73], propos uma classe de grafos com custos associados não negativos e para os quais o desempenho $A^{*}$ é exponencial. Um exemplo de um grafo desta classe é o seguinte : 
Capítulo 4: $O$ algoritmo $A^{*}$ e sua relaçäo com dualidade

Seja $G \equiv(N, G)$ o seguinte grafo

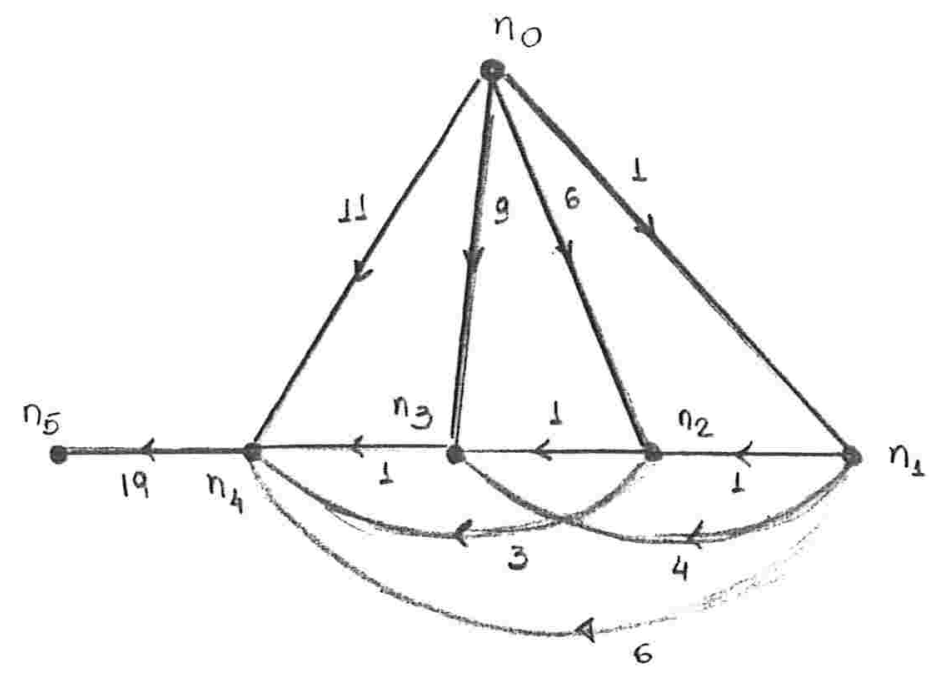

Admitindo-se as estimativas invariantes com as iterações

$\hat{h}\left(n_{0}\right)=23 \hat{h}\left(n_{1}\right)=13 \quad \hat{h}\left(n_{2}\right)=7 \hat{h}\left(n_{3}\right)=3$

$\hat{h}\left(n_{4}\right)=0 \quad \hat{h}\left(n_{5}\right)=0$

pode-se verificar que o comportamento de $A^{*}$ é exponencial,no número de nós do grafo $(0(2 \mathrm{p}))$, o que se deve basicamente ao fato de ser necessário reabrirmos nós fechados.

0 papel exercido por consistência fica claro neste ponto.Como sob a hipótese de consistência ao fechar um nó não mais o reabrirmos é de se esperar que a complexidade do algoritmo neste caso seja polinomial número de nós do grafo.Na verdade,temos

\section{TEOREMA.4.2.2}

Seja $G \equiv(N, E)$ um grafo nas condições do teorema 4.1.5 (que asseguram a finitude de $A^{*}$ ).

Se $A^{\prime \prime}$ ao ser aplicado a este grafo utiliza uma sequência de estimadores consistentes e admissíveis,então o algoritmo possui rapidez pessimista da ordem $O\left(p^{2}\right)$.

Demonstração 
Omitida pela sua semelhança com o caso do algoritmo de Dijkstra

Como consistência,não é uma propriedade trivialmente obtida,é usual encontrarmos trabalhos que procuram fager adaptaçōes no algoritmo $A^{*}$ buscando diminuir o grau de complexidade do mesmo.Nessa linha encontramos os trabalhos de Martelli [Ma77]já citado,e o de Bagchi e Mahaniti [BM83], que utilizam limitantes superiores do custo do caminho mínimo no processo de escolha de nós para fechamento,obtendo com isso uma acelereção do metódo. 0 trabalho de Gelperin [Ge77], onde se permite que as estimativas $h^{k}\left(\right.$.) dependam do estado da busca, isto é,de $g^{k}($.$) cabe$ ser o trabalho de Pearl [Pe 33] também não pode ser omitido e lá encontramos inclusive um estudo bastante detalhado sobre a complexidade média de $A^{*}$,inclusive nos casos em $\hat{h}(n)$ é uma variável randômica.

\section{3 - O ALGORITMO A* E SUA RELACÃO COM DUALIDADE}

Ao estudarmos os algoritmos de Ford e Dojkstra no capitulo precedente pudemos relacioná-los com dualidade,no sentido clássico através das funçōes $f^{k}($.$) ,que fornecem variáveis dual viáveis para o problema do cam-$ inho mais custo. 0 algoritmo $A^{*}$, sendo uma "generalização" do algoritmo de Dijkstra,deve, de certa forma estar correlacionado com dualidade,quer no sentido clássico,quer sob um conceito mais amplo.

Em termos de generalizaçâo do conceito de dualidade vamos encontrar o trabalho de Tind and Wolsey [TW81] que propos um conceito de problema dual mais geral,onde os elementos do conjunto de pontos viáveis são funçōes e não vetores de $\mathbb{R}^{p}$.0s resultados mais relevantes para nosso caso podem ser encontrados no apêndice $\Pi, 0$ q quais para efeito deste capítulo adotaremos como conhecidos.

Como sabemos,o maior problema ao estudarmos a generalização do conceito de problema dual reside na escolha do conjunto de funçöes sobre os quais se efetua a maximização. 0 dual generalizado mais conhecido ésem dúvida, o chamado DUAL SUBADITIVO,onde as funçôes consideradas são subaditivas, (isto é,satisfazem $\psi(\alpha+\beta) \leq \psi(\alpha)+\psi(\beta) \forall(\alpha, \beta)$ $\in Y x Y$, com $\psi: Y \rightarrow \mathbb{R}$ ).Mais precisamente,no caso do problema de caminho mínimo temos : 
Capítulo 4: $O$ algoritmo $A^{*}$ e sua relação com dualidade

\section{DEFINIÇÄO 4.3.1}

Chamamos de DUAL SUBADITIVO para 0 algoritmo para o problema de caminho mínimo a

(DSUB) $\sup _{\psi(.) \in U_{s a b}} \psi\left(e^{1}-e^{p}\right)$

$U_{\text {sub }}=\left\{\psi(.) \in \Im_{a} u b \mid \psi(M x) \leqq<c, x>\forall x \geqq 0\right.$

$\left.x \in \mathbb{R}^{q}\right\}$

$$
\Im_{\text {sแb }}=\left\{\psi: \mathbb{R}^{\mathrm{p}} \overleftarrow{\mathbb{R}} \mid \psi(.)\right.
$$

é subaditiva $\}$

onde $\mathrm{M}$ denota a matriz de incidência do grafo considerado.

$\mathrm{Da}$ estrutura da matriz $\mathrm{M}$ e da subaditividade das funçóes $\psi($. pode-se verificar trivialmente que esse dual é equivalente a outro problema.

\section{LEMA 4.3.2} é equivalente a

O dual subaditivo para o problema de caminho mínimo (DSUB*

$\left(D S U B^{*}\right) \sup _{\psi(.) \in U_{\text {oub }}} \psi\left(e^{1}-e^{p}\right)$

$U_{s u b}=\left\{\psi(.) \in \Im_{s} u b \mid \psi\left(e^{i}-e^{j}\right) \leqq c(i, j) \forall(i, j) \in E\right\}$

$\Im_{\text {sub }}$ como na definição 4.3.1

\section{Demonstração} deste trabalho

O resultado é um caso particular do teorema $\mathrm{A2}$ - 10 do apêndice

Se este problema por um lado restringe o conjunto de funçöes con. sideradas de outro assegura a validade de um teorema forte de dualidade (uma vez que a função perturbação do problema original

$$
v: \mathbb{R}^{\mathrm{p}} \rightarrow \mathbb{R}
$$




$$
v(y)=\inf _{x \in X_{m i n} y}\langle e, x\rangle
$$

pertence a $U_{\text {aub }}$ )

Poderíamos então tentar encontrar algum outro problema dual que, assegurando a valiadde do teorema forte de dualidade, não dificulte a determinação das funções consideradas. Assim definimos o seguinte problema.

\section{DEFINIÇÃO 4.3.3} mínimo a :

Chamamos de DUAL ESTIMADOR para o problema do caminho

$$
\begin{aligned}
& (\mathrm{DEST}) \sup _{\psi(.) U_{e a t}} \psi\left(e^{\mathbb{1}}-e^{p}\right) \\
& U_{e s t}=\left\{\psi(.) \in \Im \mid \psi\left(e^{i}-e^{j}\right) \leqq: k(i, j) \forall(i, j) \in N \times N\right\} \\
& \Im=\left\{\psi: \mathbb{R}^{\mathrm{p}} \rightarrow \mathbb{R}\right\}
\end{aligned}
$$

Observe que o dual estimador não é exatamente um caso particular do dual geral,pois as restriçōes consideradas foram impostas no conjunto de pontos viáveis e näo no conjunto de funçōes. Todavia o problema mantém as propriedades mais importantes do dual geral.

\section{LEMA 4 .3.4}

Para o problema do caminho mínimo e seu dual estimador vale o teorema fraco de dualidade, isto é,

$$
\sup _{\psi(.) \in U_{e s t}} \psi\left(e^{1}-e^{p}\right) \leqq \cdot \inf _{x \in X_{\text {min }}}\langle c, x\rangle
$$

\section{Demonstração}

Segue trivialmente da condição $\psi\left(e^{i}-e^{j}\right) \leq k(i, j)$ 
A função perturbação do problema do caminho mínimo é viável nos problemas duais subaditivo e estimador, isto é,

$v: \mathbb{R}^{\mathrm{P}} \rightarrow \mathbb{R}$

$v(y)=\inf _{x \in X ;}\langle c, x\rangle$

$\operatorname{com} X_{y}^{\prime}==\left\{x \in \mathbb{R}^{\mathrm{q}} \mid \mathrm{Mx}=\mathrm{yx} \geq 0\right\}$

satisfag i)

$$
v(.) \in U_{e s t}
$$

ii)

$$
v(.) \in U_{\text {oub }}
$$

Demonstração

i) Seja $(i, j) \in N \times N$

Se $\mathbf{k}(i, j)=+\infty, v\left(e^{i}-e^{j}\right) \leq k(i, j)$

Caso contrário, do teorema 1.2.11, a cada caminho $P^{i j}$ entre os nós i e j, $\exists x^{*} \in X_{e^{i}-e^{j}} l \quad\left\langle c, x^{*}\right\rangle=c\left(P^{i j}\right) \log 0 v\left(e^{i}-e^{j}\right) \leq c\left(P^{i j}\right) \mathrm{e}$ portanto $v\left(e^{i}-e^{j}\right) \leq k(i, j)$ Como $v(.) \in \Im_{\text {segueaa firmacäo }}$

ii) Seja $(i, j) \in E$

Claramente, $v\left(e^{i}-e^{j}\right) \leq c(i, j)$ (pois $e^{i}-e^{j}$ é uma coluna da matriz M).Além disso, a função $\mathrm{v}($.$) é subaditiva e portanto v(.) \in \Im_{\text {sub }}$.

Segue então a afirmação que $v(.) \in U_{s e b}$

Esse resultado nos mostra que os duais estimador e subaditivo são sempre viáveis e tem como corolários :

\section{COROLÁRIO 4.3.6}

Para o problema do caminho mínimo (PCMIN) e seu dual estimador (DEST) vale o teorema forte de dualidade. 
Capítulo f: $O$ algoritmo $A^{*}$ e sua relação com dualidade

Para o problema do caminho mínimo (PCMIN) e seu dual subaditivo vale o teorema forte de dualidade.

O dual estimador é menos restritivo que o subaditivo pois não impõe restrições no conjunto de funçōes utilizada,e isso certamente facilita sua construção. ( veja que pode não ser trivial o processo de construção de funções subaditivas)

\section{LEMA 4.3.8}

Nas condiçôes das definições 4.3.1 e 4.3.3 vale

i) $U_{s u b} \subset U_{e s t}$

ii) $\sup _{\psi(.) \in U_{o u b}} \psi\left(e^{1}-e^{p}\right)=$

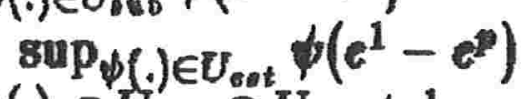

iii) $\exists \psi^{*}(.) \in U_{s u b} \cap U_{\text {est }}$ tal que $\psi^{*}($.$) resolve$

(DEST) e (DSUB)

Demonstracão

i) Seja $\psi(.) \in U_{\text {oub }}$ e $\left(\mathrm{i}_{2} \mathrm{j}\right) \in N x N$

Tomemos, caso exista, $\mathrm{P} \equiv\left(i_{1}, \ldots, i_{r}\right)$ um caminho qualquer entre $i_{1}=i$ e $j=i_{r}$

$$
\begin{aligned}
\psi\left(e^{i}-e^{j}\right) & =\psi\left(e_{1}^{i}-e_{r}^{i}\right)= \\
& =\psi\left(e_{1}^{i}+\sum_{k=2}^{r-1}\left(-E_{i k}+e_{i k}\right)-e_{r}^{i}\right)= \\
& =\psi\left(\sum_{k=1}^{r-1}\left(e_{i k}-e_{i k+1}\right)\right) \\
& \left.\vdots \sum_{k=1}^{p-1} \psi\left(e_{i k}-e_{i k+1}\right)\right)
\end{aligned}
$$

(da subaditividade de $\psi($.$) )$

Como $\forall k 1 \leqq k \leqq r-1\left(i_{r}, i_{r+1}\right) \in E$

$\psi\left(e_{i_{k}}-e_{i_{k+1}}\right) \leqq c_{i_{k}, i_{k+1}}$, e portanto

$\psi\left(e_{1}^{i}-e_{k}^{i}\right) \leqq \sum_{k=1}^{r-1} c_{i_{k}, i_{k+1}}=c\left(P^{i} j\right)$

Sendo $P^{i j}$ um caminho qualquer entre i e j,vale

$\psi\left(e^{i}-e^{j}\right) \leqq \mathrm{k}(\mathbf{i}, \mathbf{j})$.

Caso o caminho $P^{i j}$ não exista,, $\mathrm{h}(\mathrm{i}, \mathrm{j})=+\infty$ e trivialmente $\psi\left(e^{i}-e^{j}\right) \leqq: k(i, j)$ 
Capítulo 1: $O$ algoritmo $A^{*}$ e sua relação com dualidade

$\log 0 \psi(.) \in U_{e s t}$ e segue a afirmação.

ii) Basta observar que

$\sup _{\psi() \in U_{a s b}} \psi\left(e^{1}-\quad\right)=v\left(e^{1}-e^{p}\right)=\sup _{\psi(.) \in U_{a \theta t}} \psi\left(e^{1}-e^{p}\right)$

iii) Imediato,tomando-se $\psi()=.v(.) \in\left(U_{s u b} \cap U_{e s t}\right)$

No capitulo anterior vimos que as funções determinavam a escolha de nós para fechamento do algoritmo correspondiam a variáveis dual viáveis.Aqui,não s somente essa relação é valida como também uma "reciproca" do mesmo que nos permite ,a partir de funçãos dual-viáveis construir subestimadores para 0 algoritmo $A^{*}$.

Vamos admitir inicialmente que seja conhecida uma formula de subestimadores de caminho mínimo $\hat{h}_{i}({ })_{i \in N}$ que sejam estimadores,isto é,

$$
\begin{aligned}
& \hat{h}_{i}: N \rightarrow \mathbb{R} \forall i \in \mathbb{N} \\
& \hat{h}_{i}(j) \leq k(i, j) \forall(i, j) \in N \times N
\end{aligned}
$$

ou seja,adotando uma notaçấo mais simples consideremos

\section{DEFINIÇÄO 4.3 .9}

Dizemos que uma função $\hat{h}(.,$.$) satisfaz a propriedade de admissi-$ bilidade se

$$
\begin{aligned}
& \hat{h}: N \times N \rightarrow \mathbb{R} \\
& \hat{h}(i, j) \leqq k(i, j) \forall(i, j) \in N \times N
\end{aligned}
$$

\section{PROPOSIÇÃO 4.3.10}

Suponhamos que se conheça uma sequência de funções $\left\{h^{k}(., .)\right\}_{k \in \mathbb{N}}$ satisfazendo a propriedade de admissibilidade.Então definindo-se

$$
\psi^{k}: \mathbb{R}^{\mathrm{p}} \rightarrow \mathbb{\mathbb { R }}
$$

* convenciona-se que $\forall m \in \mathbb{R}-\infty+m=-\infty$ 
Capítulo 4: $O$ algoritmo $A^{*}$ e sua relação com dualidade

$\psi^{k}(y)= \begin{cases}h^{k}(i, j) & \text { se } y=e^{i}-e^{j}(i, j) \in N x N \\ -\infty & \text { c.c }\end{cases}$

temos que $\forall k \in \mathbb{N} \psi^{k} \in \mathrm{U}_{\text {est }}$

\section{Demonstração}

Imediata da definição de dual estimador

Uma primeira correlação entre $A^{*}$ e dualidade pode ser obtida : o algoritmo utiliza subestimadores $h^{k}($.$) adimissíveis,e portanto,$

$\hat{W}^{k}(i, j)= \begin{cases}\mathrm{h}^{k}(i) & \text { se } \mathrm{j}=\mathrm{p} \forall(i, j) \in N \mathrm{xN} \\ -\infty & \text { c.c }\end{cases}$

estamos nas condições da proposição anterior e podemos construir trivialmente uma função dual viável.Essa sequência de funçōes,no entanto,não possui propriedades muitos fortes a menos que se imponha condiçóes mais restritivas sobre $\left\{h^{k}().\right\}$

\section{DEFINIÇÄO 4.3.11}

Dizemos que uma função $\mathrm{h}(.,$.$) satisfaz a propridade de consitência$ se $\hat{h}: N \times N \rightarrow \mathbb{R}$ obedece as condiçôes

i) $\hat{h}(i, i)=0 \forall i \in N$

ii) $\hat{h}(i, j) \leqq k(i, l)+\hat{h}(l, j) \forall(i, l, j) \in N \times N \times N$

\section{PROPOSIÇÃO 4.3.12}

Seja $\left\{h^{k}(., .)\right\}_{k \in \mathbb{N}}$ uma sequência de funçōes satisfarendo a propriedade de consistência. Então sendo $\left\{f^{k}(.)\right\}_{k \in \mathbb{N}}$ a sequência de funções gerada por $\boldsymbol{A}^{*}$ ao utilizar os subestimadores $\left\{h^{k}(,, p)\right\}_{k \in \mathbb{N}}$ tem-se que a sequência de funções

$$
\begin{aligned}
& \psi^{k}: \mathbb{R}^{p} \rightarrow \overline{\mathbb{R}} \\
& \psi^{k}(y)= \begin{cases}\max _{l \in C^{k}} f^{k}(l) & \text { se } y=e^{1}-e^{p} \\
h^{k}(i, j) & \text { se } y=e^{i}-e^{j}(i, j) \in N \times N(i, j) \neq(1, p) \\
-\infty & \text { c.c }\end{cases}
\end{aligned}
$$

satisfar 
Capítulo 1: $O$ algoritmo $A^{*}$ e sua relação com dualidade

$\forall k \in N \psi^{g} \in U_{e s t}$

\section{Demonstração}

Se $\mathrm{y} \neq e^{1}-e^{p}$, trivialmente $\psi\left(e^{i}-e^{j}\right) \leq=\mathrm{k}(\mathrm{i}, \mathrm{j}) \mathrm{da}$ definição de $U_{\text {est }}$ Se $y=e^{1}-e^{p}$, então como a sequência de estimadores $\left\{h^{k}(,, p)\right\}_{k \in \mathbb{N}}$ é consistente (no sentido usual), temos que $\forall l \in C^{k} f^{k}(l) \leq=g(p)=k(1, p)$ Assim,

$\psi^{q}\left(e^{1}-e^{p}\right)=\max _{l \in C^{k}} f^{k}(l) \leq=\max _{l \in C^{k}} k(1, p)=k(1, p)$

Logo.

$\forall(i, j) \in N \times N \psi^{k}\left(e^{i}-e^{j}\right) \leq=k(i, j)$ e dai, $\psi^{k} \in U_{e s t}$

Com esse resultado mostramos que o critério de fechamento dos nós de $A^{*}$, quando utilizando uma heurística bem comportada,permite que se construa funçôes dual viáveis,reproduzindo resultados válidos para o algoritmos de Dijkstra e Ford. Esse resultado é ainda mais forte

\section{TEOREMA 4.3.13}

Nas condições da proposição anterior sendo $k^{*}$ a iteração na qual o algoritmo termina,tem-se que se $\mathrm{p} \in C^{k^{*}} \psi^{k^{4}}($.) é solução ótima do dual estimador (DEST)

\section{Demonstração}

Do resultado anterior $\psi^{k^{*}}(.) \in U_{\text {est }}$

Além disso, $\psi^{k^{*}}\left(e^{1}-e^{p}=\max _{l \in C^{*}} g^{k^{*}}(l)+h^{k^{*}}(l, p) \geq=\right.$

$g^{k^{*}}(p)+h^{k^{*}}(p, p)=g^{k^{*}}(p)=k(1, p)$, ou seja,

$\psi^{k^{*}}\left(e^{1}-e^{p}\right) \geq=k(1, p)$.

Da viabilidade de $\psi^{k}($.$) segue entảo que \psi^{k^{*}}\left(e^{1}-e^{p}\right)=k(1, p)$ e portanto $\psi^{k^{*}}($.$) é solução ótima de (DEST).$

Observe que se na iteração de $k^{*}$ o nó $\mathrm{p} / C^{k^{*}}$ o problema original é inviável e portanto $v\left(E^{1}-e^{p}\right)=+\infty$.Como v(.) é uma soluçäo ótima do dual estimador,segue então que o valor ótimo de (DEST) é $v^{*}=+\infty$. 
Capítulo 4: $O$ algoritmo $A^{*}$ e sua relação com dualidade

Claramente outras funções podem ser construidas utilizando-se o algoritmo, cmo mostra o seguinte resultado :

\section{LEMA 4.2.14}

Nas condições da proposição anterior a sequência de funções definida por

$\psi^{k}: \mathbb{R}^{p} \rightarrow \mathbb{R}$

$\psi^{k}(y)= \begin{cases}\min _{l \in C^{k}}\left\{g^{k}(l)+h^{k}(l, j)\right\} & \text { se } y=e^{1}-e^{j} \\ \mathrm{~h}^{k}(i, j) & \text { se } y=e^{i}-e^{j}(i, j) \in N \times N k \neq 1 \\ -\infty & \text { c.c. }\end{cases}$

$\forall k \in \mathbb{N}, \psi^{\mathbb{k}}(.) \in \mathrm{U}_{\text {est }}$

\section{Demonstração}

Claramente,sendo y $\in \mathbb{R}^{\mathrm{p}}, \mathrm{y}=\mathbf{e}^{1}-\mathbf{e}^{\mathrm{P}}$ temos

$\min _{e \in C^{k}} g^{k}(e)+h^{k}(l, j) \leqq g^{k}(1)+h^{k}(1, j)=0+h^{k}(1, j) \leqq$

$\mathrm{k}(1, \mathrm{j})$ e caso $\mathrm{y}=e^{i}-e^{j}(\mathrm{i}, \mathrm{j})(\mathrm{i}, \mathrm{j}) \in N \mathrm{x} N i \neq 1$ temos

$\psi^{k}\left(e^{i}-e^{j}\right)=h^{k}(i, j) \leqq \mathbf{l}(\mathrm{i}, \mathrm{j})$

O maior problema ao utilizarmos essa funçäo reside na determina. ,ão de $h^{k}(.,$.$) ,pois não é verdade ,a priori, que a término do processo \psi^{k^{*}}($. é solução ótima de (DEST).Essa construção no entanto nos será útil quando estudarmos a aplicação de $A^{*}$ a problema mais gerais que o problema do caminho mínimo.

Observe que os resultados vistos não se aplicam ao caso de dualidade subaditiva, pois as funçōes construidas não são necessariamente subaditivas como mostra o seguinte exemplo:

\section{EXEMPLO 4.2.15}

Considere o grafo $\mathrm{G} \equiv(\mathrm{N}, \mathrm{E})$ 
Capítulo f: $O$ algoritmo $A^{*}$ e sua relação com dualidade

Sendo $\hat{h}: N x N \rightarrow \mathbb{R}$

e

$\hat{h}(i, j)= \begin{cases}2 & \text { se }(i, j) \\ 0 & \text { c.c }\end{cases}$

' de fácil verificaşão que $\hat{h}(.,$.$) satisfaz a hipótese de consistência.$

A função $\psi^{1}($.$) serão então$

$\psi^{1}(y)=$

$\max _{e \in\{1,4\}}\left\{g^{1}(e)+\hat{h}(e, 4)\right\} \quad y=e^{1}-e$

$\hat{h}(i, j) y=e^{i}-e^{j}(i, j) \in N x N$

$-\infty$ c.c

e teremos $2=\psi^{1}\left(e^{1}-e^{4}\right)=\psi^{1}\left(e^{1}-e^{3}+e^{3}-e^{4}\right)>$

$\psi^{1}\left(e^{1}-e^{3}\right)+\psi^{1}\left(e^{3}-e^{4}\right)=0$

não sendo então subaditiva.

A relação entre $A^{*}$ e dualidade não se restringe à construção de variáveis viáveis no problema dual. Vale uma "reciproca" das resultados vistos,que nos permite caracterizar funções viáveis nos problemas duais como "candidatos" a subestimadores de caminho mínimo.

\section{PROPOSIÇÃO 4.2.16}

Seja $\psi(.) \in U_{\text {est }}$ uma função viável no dual estimador.Então $\psi($. indus uma de subestimadores de caminho mínimo admissível dado por

$$
\begin{aligned}
& \hat{h}: N \rightarrow \mathbb{R} \\
& \hat{h}(j)=\psi\left(e^{j}-e^{p}\right)
\end{aligned}
$$

Demonstração

Trivial

Esses estimadores não são necessariamente consistentes sendo para tanto que se imponha mais condições sobre as funçōes $\psi($.$) .$ 
Capítulo f: $O$ algoritmo $A^{*}$ e sua relação com dualidade

Seja $\psi(.) \in U_{s u b}$, uma função viável no dual subaditivo. Então o subestimador induzido por $\psi($.$) conforme proposição anterior e admissível.$

Demonstração

Imediata pois $U_{\text {sub }} \subset U_{\text {est }}$

\section{LEMA 4.2.18}

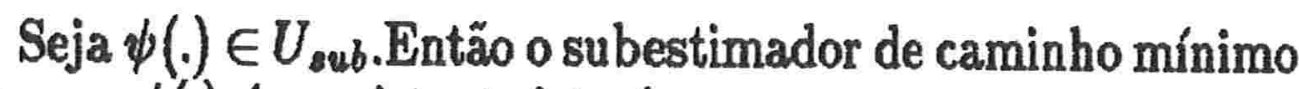
induzido por $\psi($.$) é consistente, isto é,$

$$
\forall(i, j) \in N \times N \hat{h}(i) \leqq k(i, j)+\hat{h}(j)
$$

Demonstração

$\forall(i, j) \in N \times N$ tem-se

$\hat{h}(i)=\psi\left(e^{i}-e^{p}\right)=\psi\left(e^{i}-e^{j}+e^{j}-e^{p}\right) \leqq \psi\left(e^{1}-e^{j}\right)+\psi\left(e^{j}-e^{p}\right)$ da subaditividade de $\psi($.)

Como $U_{\text {sub } b} \subset U_{\text {est }} \psi\left(e^{i}-e^{j}\right) \leqq \mathrm{k}(\mathrm{i}, \mathrm{j})$ e consequentemente $\hat{h}(i) \leq k(i, j)+\psi\left(e^{i}-e^{p}\right)=k(i, j)+\hat{h}(j)$

e (h)'eonsistente

Esse conjunto de resultados nos fornece uma maneira bastante simples de obter estimadores admissíveis e consistentes para o algoritmo $A^{*}$, bastando que tomemos elementos ví́veis no dual subaditivo. Fica assim estabelecida uma relação bastante forte dualidade e o algoritmo $A^{*}$.

\subsection{CONCLUSÃO}


Uma das razóes pelas quais elaboramos um estudo tão detalhado,e, consequentemente tão extensão sobre $A^{*}$, se deve basicamente a importância do algoritmo não somente em termos operacionais mas também à sua relevância enquanto algoritmo dual.

Mais do que a criação de uma linha de trabalho para a determinação de "bons estimadores" para 0 algoritmo,obtvemos,conforme será vistos adiante, um conjunto ou ferramentas para a resolução de problemas mais complexos como problemas de grupos,entre outros.Naturalmente o algoritmo $A^{*}$ aqui estudado se aplica aqueles problemas seja soluçảo pode ser determinada através da resolução de um problema busca de caminho mínimos em grafos : desde que possamos fazer tal transformaçãa de maneira eficiente,teremos um método eficar de resolução do problema original.

Nosso objetivo a partir de agora passa a ser estudo da utilização de $A^{*}$ para a resoluçáo de uma outra classe de problemas de grupos. 


\section{Capítulo 5}

\section{Problemas de grupos}

Por um problema de programação linear inteira ou simplesmente,um problema inteiro,compreende-se um problema de programação linear com a restrição de que as componentes de que qualquer ponto viável sejam inteiro.

Um caso típico de tal problema ocorre quando extendemos o conceito de programação linear a grupos comutativos,isto é, quando tratamos os coeficientes da matriz de restriçōes, bem como as componentes do vetor de termos independentes como elementos de um grupo comutativo.Neste caso,o produto de um elemento do grupo $a_{i j}$,por um inteiro não negativo, $x_{j}$ corresponde a soma de $a_{i j}$ a si mesmo $x_{j}$ vezes,e portanto e razoável considerarmos a restrição, $A x=b x \geqq 0 x$ inteiro.

Este problema, que chamaremos problema geral de grupos, possui inúmeras aplicações e tem como desvantagem a alta complexidade dos métodos para sua resolução, o que justifica a busca de métodos mais efi- 
cientes para resolvê-los.

Nos capítulos precedentes vimos que para o caso particular do problema do caminho mínimo, dispunhamos de bons algoritmos que utilizavam a estrutura de grafos inerente a ele.Como a problemas definidos sobre grupos pode-se associar um grafo para o qual a deteç̧ão de um caminho mínimo e equivalente a resolução do problema original, é natural tentarmos resolver - problema de grupo aproveitando a estrutura do grafo correspondente.

Neste capítulo, além de apresentarmos a transformação do problema de grupos em um problema de determinação de caminhos mínimos , exploraremos características do grafo obtido, que derivam do fato de estarmos trabalhando com grupos.Mais ainda, procuraremos mostrar o comportamento dos algoritmos para a resolução de problemas de caminhos mínimos, quando utilizados para a resolução do problema de grupos.

\section{1 - O PROBLEMA DE PROGRAMAÇÄO LINEAR INTEIRA E UM PROBLEMA DEFINIDO SOBRE GRUPOS}

Definamos formalmente o problema inteiro e de grupos:

\section{DEFINIÇÃO 5.1 .1}

$O$ problema de programação linear inteira, $(\mathrm{PI})$, consiste em:

Dados $c \in \mathbb{R}^{q}, A \in \mathbb{Z}^{p \times q} e b \in \mathbb{Z}^{q}$, encontre se existir ,

$$
x^{*} \in \operatorname{argmin}_{x \in X_{P I}}<c, x>
$$

onde,

$$
X_{P I}=\left\{x \in \mathbb{R}^{q} \mid A_{x}=b x \geqq 0 x_{j} \in \mathbb{Z} \quad \forall \in\{1,2, \ldots, q\}\right\}
$$

isto é,

$$
X_{P I}=\left\{x \in \mathbb{Z}^{q} \mid A x=b x \geqq 0\right\}
$$


Devido a ausência de convexidade do conjunto de pontos viáveis , não é possível, em geral , a utilização de algoritmos como o simplex para a resolução de (PI), a menos de casos particulares onde a relaxação da restrição $x \in \mathbb{Z}^{q}$ conduz a problemas cuja solução ótima permanece inalterada.

Algumas das abordagens utilizadas para a resolução do problema (PI) são a utilização de métodos de planos de corte consistem na construção iterativa de equações, a partir de soluções do problema relaxado (isto é , ao invés de $x \in \mathbb{Z}^{q}$ considerarmos $x \in \mathbb{R}^{q}$ ) que são adicionadas ao problema original.Essas restrições, chamadas cortes, são geradas de tal forma que o problema obtido ao adicioná-las tenha a mesma solução ótima que o problema original .0 trabalho clássico dentro dessa linha e de Gomory [GO65], aplicável a qualquer problema inteiro.

A resolução de (PI) em termos de grupos também procura gerar cortes, através da solução de um problema de minimização, definido sobre um problema auxiliar de grupos, cuja solução é obtida através de algoritmos específicos . Esse tipo de abordagem pode ser relacionada também a trabalhos de Gomory.

A importância destes problemas definidos sobre grupos, subjacentes ao problema inteiro, não se restringe a solução de (PI) mas a sua própria estrutura,razão pela qual nos concentraremos no seu estudo.Para que fique clara a relação entre (PI) e o problema definido sobre grupos , apresentamos suscintamente a construção deste último a partir de um problema inteiro.

A relaxação mais simples de um problema linear inteiro e dada por

\section{DEFINIÇÄO 5.1 .2}

Dado um problema de programação linear inteira (PI) , definimos a RELAXAÇÃO USUAL DE (PI), como :

(PREL) Encontre se existir,

$$
x^{*} \in \arg \min _{x \in X_{R E L}}\langle c, x\rangle
$$

, onde , 


$$
X_{R E L}=\left\{x \in \mathbb{R}^{q} \mid \mathrm{Ax}=\mathrm{b} \mathrm{x} \geqq 0\right\}
$$

Fixada uma base viável B desse problema, sem perda de generalidade $\mathrm{B}=(1,2, \ldots, p)$, podemos reescrever o conjunto de pontos viáveis do problema inteiro, $(\mathrm{PI})$, da seguinte forma

$$
\begin{aligned}
& X_{P I}=\left\{x \in \mathbb{R}^{\mathrm{q}} \mid \mathrm{x}_{\mathrm{B}}=\mathrm{A}_{\mathrm{B}^{-1} \mathrm{~b}-\mathrm{A}_{\mathrm{B}^{-1}} \tilde{\mathrm{A}} \tilde{\mathrm{x}}}\right. \\
& x_{B} \geqq 0 \text { inteiro } \\
& \text { onde } \left.x=\left(x_{B} \mid \tilde{x}\right) \quad A=0 \text { inteiro }\right\}
\end{aligned}
$$

Com isso o problema (PI) se reescreve como

$$
\text { (P1) } \min \left\langle c_{B}, A_{B}^{-1} x_{B}\right\rangle+\langle\tilde{y}, \tilde{x}\rangle
$$

$$
\begin{aligned}
& \text { sa } x_{B}=A_{B}^{-1} b-A_{B}^{-1} \tilde{A} \tilde{x} \\
& x_{B} \geq 0 \text { inteiro }
\end{aligned}
$$$$
\tilde{x} \geq 0 \overline{0} \text { inteiro }
$$

onde $\tilde{\gamma}=\tilde{c}-\tilde{A}^{t} A_{B}^{-1} c_{B}, \operatorname{com} c=\left[c_{B} \mid \tilde{c}^{t}\right]$

Para que construamos um problema definido sobre grupos a partir deste último, utilizaremos a operação congruência módulo 1 , definida da seguinte forma:

\section{DEFINIÇÃO 5.1.3}

Dados $(x, y) \in \mathbb{R} x \mathbb{R}$, dizemos que $\mathrm{x}$ é congruente a y modulo $\mathbf{k}$, $k \in \mathbb{N}(\mathrm{x} \equiv \mathrm{y}(\operatorname{modk}))$, se $\exists r \in \mathbb{Z} \mid x-y=r * k$

No caso em que $(x, y) \in \mathbb{R}^{q} \times \mathbb{R}^{q}$, dizemos que $x$ é congruente a y modulo $\mathrm{k}(x \equiv y(\bmod k))$ se

$$
\forall i \in\{1,2, \ldots, q\} \quad x_{i} \equiv y_{i}(\bmod k)
$$

É claro, segundo essa definição, que qualquer elemento viável em (PI) satisfaz então

* Nesse ponto estaremos adotando, assim como o fizemos no capitulo 1, uma notação consistentes com o trabalho de Humes[HH86] 


$$
x_{B} \equiv A_{B}^{-1} b-A_{B}^{-1} \tilde{A} \tilde{x}(\bmod 1)
$$

Mas $x_{B}$ é inteiro se e só se $x_{B} \equiv 0(\bmod 1)$ e portanto qualquer elemento viável em (PI) satisfaz

$$
\begin{aligned}
A_{B}^{-1} b \equiv & A_{B}^{-1} \tilde{A} \tilde{x}(\bmod 1) \\
& \tilde{x} \geqq 0 \text { inteiro } \\
& x_{B}=A_{B}^{-1} b-A_{B}-1 \tilde{A} \tilde{x} \geqq 0
\end{aligned}
$$

Retornando ao problema (P1),observe que a função objetivo independe da constante $\left\langle c_{B}, A_{B}^{-1} b\right\rangle$ e portanto as soluçóes ótimas de (PI) resolvem então

P2 $\min \langle\tilde{\gamma}, x\rangle$

bem como

$$
\begin{aligned}
& \text { sa } A_{B}^{-1} \tilde{A} \tilde{x} \equiv A_{B}^{-1} b(\bmod 1) \\
& \tilde{x} \geqq 0 \text { inteiro } \\
& x_{B}=A_{B}^{-1} b-A_{B}^{-1} \tilde{A} \tilde{x} \geqq 0
\end{aligned}
$$

P3 $\min \langle\tilde{\gamma}, x\rangle$

$$
\begin{aligned}
& \text { sa } A_{B}^{-1} \tilde{A} \tilde{x} \equiv A_{B}^{-1} b(\bmod 1) \\
& \tilde{x} \geqq 0 \text { inteiro }
\end{aligned}
$$

Note que não necessariamente a partir de soluções $\tilde{x}$ desse problema se pode obter solução de (PI) pois não necessariamente o correspondente $x_{B}$ é não negativo.

Em termos operacionais podemos resolver o problema relaxado (Prel),detectando-se inviabilidade do mesmo, ou uma base ótima $B^{*}$.Se esta base corresponder a um vetor inteiro,o problema (PI) terá sido resolvido * Caso contrário,resolve-se o problema (P3) obtendo-se uma solução $\tilde{x}^{*}$ tal que se $x_{B} \geqq 0$ então $\left(x_{b} \mid \tilde{x}\right)$ resolve $(\mathrm{PI})$.

Sendo $F=\left[A_{B^{-1}} \mid \tilde{A}\right]$ e $f^{0}=A_{B}^{-1} b$,onde $\mathrm{B}$ e uma base ótima da relaxação,temos que o problema e um caso particular de:

Dados $d \in \mathbb{R}^{\mathrm{n}}, \mathbf{F} \in \mathbb{R}^{\mathrm{m} \times \mathrm{n}}$ e $f^{0} \in \mathbb{R}^{\mathrm{m}}$,encontre se existir

* Esses resultados se baseiam no seguinte lema:

LEMA: Sendo $v^{*}=\inf _{x \in X_{P I}}\langle c, x\rangle e v_{r}^{*}=\inf _{x \in X_{\text {rel }}}\langle c, x\rangle$, os valores ótimos dos problemas (PI) e sua relaxação usual, vale

i) $v_{R}^{*} \in \mathbb{R} \rightarrow v^{*} \in \mathbb{R} \cup\{+\infty\}$

ii) $v_{R}^{*}=+\infty \Rightarrow v^{*}=+\infty$

iii) $\exists x^{*} \in \arg \min _{x \in X_{R E L}}\langle c, x\rangle \mid x^{*} \in X_{P I}$

$\Rightarrow x^{*} \in \arg \min _{x \in X_{P I}}\langle c, x\rangle$ 


$$
\begin{aligned}
& x^{*} \in \arg \min _{x \in X_{g}}<d, x> \\
& X_{g}=\left\{x \in \mathbb{R}^{\mathrm{r}} \mid \mathrm{Fx} \equiv \mathrm{f}^{0}(\bmod 1) \quad \mathrm{x} \geqq 0 \text { inteiro }\right\}
\end{aligned}
$$

Esse último problema e o ponto central do estudo apresentado nesse capítulo e nos que seguem, sendo que procuraremos estudar uma particular classe de algoritmos para sua resolução,relacionando-a com o algoritimo $A^{*}$.

\subsection{O PROBLEMA DE GRUPOS - UMA RELAÇÃO COM O PROBLEMA DE BUSCA EM GRAFOS}

De maneira geral o problema de grupos pode ser definido do seguinte modo :

\section{DEFINIÇÃO 5.2.1}

O PROBLEMA GERAL DE GRUPOS (PGG) é definido por :

Seja $G_{r} \equiv(S, \oplus)$ um grupo comutativo, $S \subset \mathbb{R}^{\mathrm{P}}$. Dados $S_{1}=$ $\left\{g_{1}, g_{2}, \ldots ., g_{q}\right\} \subset S, d \in \mathbb{R}^{\mathrm{q}}$ e $g^{*} \in S$

,encontre se existir,

$$
\begin{aligned}
& x^{*} \in \text { arg } \min _{x \in X_{G G}}<d, x>\text { onde } \\
& X_{G G}=\left\{x \in \mathbb{Z}^{q} \mid \mathbb{Q}_{i=1}^{q} g_{i} x_{i}=g^{*} x \geqq 0\right\} \text { com }
\end{aligned}
$$

$£$ denotando a somatória no grupo $G_{r}$, segundo a operação $\oplus$ e $g_{i} x_{i}=\sum_{i=0}^{x_{i}} g_{i}$

Para esse problema são conhecidos alguns métodos de resolução sendo salientada na literatura,a utilização de métodos recursivos como programação dinâmica por exempo [Hu69],[GN 72]

Um caso comum de problema de grupos ocorre quando o grupo é gerado a partir de um número finito de vetores de $\mathbb{R}^{\mathrm{p}}$ e a operação adotada e a soma modulo $1^{*}$, tal qual obtivemos no ítem anterior (5.1),ao estudarmos uma técnica para a resoluçảo de (PI).

* A operação de soma modulo 1 extendido $\mathbb{R}^{\mathrm{p}}$ é definido por

$\equiv: \mathbb{R}^{\mathrm{p}} \times \mathbb{R}^{\mathrm{p}} \rightarrow\left[0,11^{\mathrm{p}}\right.$ onde

$$
\alpha+\beta=\varsigma \Leftrightarrow \alpha+\beta \equiv \varsigma(\bmod 1)\left(\varsigma \in \left[0,1\left[^{p}\right)\right.\right.
$$


Mais precisamente,

\section{DEFINIÇÃO 5.2.2}

O problema de minimização de grupos,ou , simplesmente,PRO. BLEMA DE GRUPOS (PG) consiste em :

Dados $F \in \mathbb{R}^{\text {pxqfo }} \in \mathbb{R}^{p}$ e $d \in \mathbb{R}^{q}$,

encontre,se existir $x^{*} \in$ arg $\min _{2 \in X_{\mathbb{G}}}\langle d, x\rangle$ onde

$X_{G}=\left\{x \in \mathbb{R}^{q} \mid \mathrm{Fx} \equiv \mathrm{f}^{0} \mathrm{x} \geqq 0 \mathrm{x} \in \mathbb{Z}^{q}\right\}^{*}$

isto é, $X_{G}=\left\{x \in \mathbb{Z}^{q} \mid F x \equiv f^{0} x \geqq 0\right\}$

Trabalhando-se com a operação soma modulo 1, não há perda de generalidade em assumirmos que os coeficientes da matriz $\mathrm{F}$, bem como os de $f^{0}$ possuam componentes no intervalo [0,1[.Além disso,podemos também admitir que as colunas da matriz $\mathrm{F}$ sejam distintas (caso contrário,pode se construir um novo problema,equivalente ao original,onde as colunas iguais são substituidas por uma única $F^{j}$ com custo

$$
\hat{d}=\min _{j \in J}\left\{d_{j}\right\}, J=\left\{j \in\{1,2, \ldots q\} \mid F^{j} \equiv F^{j^{*}}\right\}
$$

Também não há perda de generalidade em assumirmos que as colunas da matriz F sejam não nulas

Além disso, para o problema de grupos,conforme definição 5.2.2,assumimos que :

$$
\begin{aligned}
& \forall i \in\{1,2, \ldots, p\} \forall j \in J=\{1,2, \ldots, q\} \\
& F_{i j} \in|0,1| e f_{i}^{0} \in[0,1]
\end{aligned}
$$

Ao considerarmos o problema de grupos (PG), estamos tentando encontrar uma combinação linear inteira formal positiva ** que nos permita obter $f^{0}$ a custo mínimo,ou seja,pretende-se obter $f^{0}$ a partir da soma,módulo 1 , dos vetores $F^{j}$ com possíveis repetições dos mesmos.De

* Por simplicidade denotaremos a operação $\alpha \equiv \beta(\bmod 1)$ por $\alpha \equiv \beta$ exceto nos casos onde tal notação se tornar ambígua.

* * dizemos que $\beta \in \mathbb{R}^{p}$ e uma combinaçã linear formal positiva dos elementos $F^{1}, F^{2}, \ldots, F^{q}$ de $\mathbb{R}^{p}$ se existem inteiros $x_{1}, \ldots, x_{q}$, não negativos tais que $\sum_{i=1}^{q} x_{i} F^{i}=\beta$ onde $\sum$ denota a somatória do módulo 1 dos elementos $\left\{F^{j}\right\}_{j=1}^{q}$ 
certa forma,esse problema se assemelha ao de determinação de caminhos mínimos em grafos no sentido de estarmos tentando "compor arcos $\left(F^{j}\right)$ a fim de atingir um fixado alvo $\left(f^{\circ}\right)$.É portanto natural verificarmos a relação entre a solução do problema de grupos com o problema de determinação de caminhos mínimos em grafos.

\section{HIPÓTESE 5.2.3}

Para o problema de grupos, conforme definição 5.2.2.,assumimos que $\forall i \in\{1,2, \ldots, p\} \forall j \in J=\{1,2, \ldots, q\} F_{i j} \in\left[0,1 \mid\right.$ e $f_{i}^{0} \in[0,1]$ sendo que $\forall\left(j_{1}, j_{2}\right) \in J \times J F^{j_{1}} / F^{j_{2}}$ CONSTRUÇÃO 5.2.4

Considere o problema de grupo (PG), e seja $G_{P G} \equiv(N, E)$ um grafo orientado com custos associados as arestas definido por :

i) $\mathbf{N}=\left\{y\right.$ in $|0,1|^{p} \mid \exists x \geqq 0: y \equiv F x$ inteiro $\}$,oconjuntodeno.

ii) $\mathrm{E}=\left\{(i, j) \in N \times N \mid \exists k \in\{1,2, \ldots q\}: j \equiv i+F^{k}\right\}$

iii) $c: E \rightarrow \mathbb{R}$

$$
c_{i j}=e(i, j)=d_{k} \text {, }
$$

onde $j \equiv i+F^{k} k \in\{1,2, \ldots, q\}$

‘Exemplificando

\section{EXEMPLO 5.2.5}

Considere o problema

$$
\operatorname{mind}_{1} x_{1}+d_{2} x_{2}
$$

$$
\begin{aligned}
& \text { sa. } 0,5 x_{1}+0.2 x_{2} \equiv 0.8(\bmod 1) \\
& x_{1} \geqq 0 x_{2} \geqq 0
\end{aligned}
$$

Neste caso, $N=$

$\{t \mid t=k / 10$ para algum $k \in \mathbb{N} 0 \geqq \mathbb{k} \geqq 10\}$ 
Primeiramente estudaremos algumas propriedades de $G_{P G}$.

\section{FATO 5.2.6} mente finito,isto é,

O grafo $G_{P G} \equiv(N, E)$ associado ao problema de grupos é local$\forall j \in N \# \operatorname{suc}(j)<\infty$

Demonstração

Imediata da construção de $G_{P G}$ observando que

$\forall j \in N \operatorname{suc}(j)=\left\{k \in N \mid \exists r 1 \leqq r \leqq q: k \equiv j+F^{r}\right\}=\bigcup_{r=1}^{q}\left\{j+F^{r}\right\}$

$e^{\prime}$ finito.

A finitude do grafo no entanto,só pode ser assegurada em alguns casos como por exemplo quando os coeficientes das restriçōes do problema forem racionais, isto é, $F \in \mathbf{Q}^{\mathrm{pxq}}$. Assim,assumiremos a seguinte hipótese:

\section{HIPOTESE 5.2.7}

$O$ problema de grupos considerado satisfaz: $\{1,2, \ldots q\}$.

$$
F_{i j} \in\left[0,1 \mid \text { hQ e } f_{i}^{0} \in[0,1 \mid \cap Q \quad \forall i \in\{1,2, \ldots p\} \forall j \in\right.
$$

Essa hipótese näo é muito restritiva se considerarmos que qualquer problema tratado através de um computador será representado através de números racionais, e, além disso, tomando $F \in Q^{p \times q}$ teremos que se 0 
Demonstração ( apesar de trivial apresentada para ilustrar o raciocínio utilizado neste tipo de resultado).

Como $\forall i \in\{1,2, \ldots p\} \forall j \in\{1,2, \ldots, q\} F_{i j} \in \mathbf{Q}$, temos que

$F_{i j}=\mathrm{s} \frac{i j}{w_{i j}}$ onde $\operatorname{mdc}\left(s_{i j}, w_{i j}\right)=1 w_{i j} \in \mathbb{N} s_{i j} \in \mathbb{Z}$.

Tomando-se $\bar{\mu}=m m c_{1 \leqq i \leqq p, 1 \leqq j \leqq q}\left\{w_{i j}\right\}$ temos que

$\forall i \in\{1,2, \ldots p\} \forall j \in\{1,2, \ldots, q\} \bar{\mu} * F_{i j} \in \mathbb{Z}$.

Sejam $S(\bar{\mu})=\left\{r \in \mathbb{Z}^{q} \mid 0 \leqq r \leqq \bar{\mu} \mathbb{H}\right\}$

e $W=\left\{y \in \mathbb{R}^{p} \mid y \equiv\right.$ Fr para algum $\left.r \in S(\bar{\mu})\right\}$, claramente finitos.

É imediato que $W \subset N$.

Por outro lado,seja $y \in N$ y $\in N \Rightarrow \exists \bar{x} \in \mathbb{Z}^{q} \bar{x} \geq 0$ tal que $y \equiv$ $F \bar{x}$.Como $\bar{x} \in \mathbb{Z}^{q}, \forall i \in\{1,2, \ldots, q\}, \exists k \in \mathbb{Z}^{q}$ e $r \in S(\bar{\mu})$ tais que $\bar{x}_{i}=$ $k_{i} \bar{\mu}+r_{i}$.Claramente, $y \equiv F \bar{x} \equiv F(k \bar{\mu}+F r) \equiv \bar{\mu} F k+F r \equiv 0+F r$ e portanto $y \in W$.

Assim, $\mathbf{N}=\mathrm{W}$ e sendo $\mathbf{N}$ finito segue a afirmação

Para que possamos relacionar o problema de grupos com o de determinação de caminhos mínimos,devemos excluir grafos que possuam arcos "paralelos".Assim assumiremos a seguinte hipotese.

\section{HIPÓTESE 5.2.9}

Para o problema de grupos considerado temos que

$$
\forall i \in\{1,2, \ldots p\} \forall j \in\{1,2, \ldots, q\} F^{i} \neq F^{j} \quad \text { e } F^{j} \neq 0 \text {. }
$$

*

Quanto a conexidade do grafo temos:

TEOREMA 5.2.10

* Note-se que esta hipótese pode ser assumida sem perda de generalidade,pois e sempre possível obter uma soluçäo ótima $x^{*}$ para o problema,tal que, $\forall(i, j) \in \operatorname{supp}\left\{x^{*}\right\} \times \operatorname{supp}\left\{x^{*}\right\} F^{i} \not \equiv F j$ 
O grafo $G_{P G}$ é fortemente conexo,isto é, $\forall(i, j) \in N \times N \exists$ um passeio $P_{i j}$ entre i e j

\section{'Demonstração}

A verificação do resultado sera feita em duas etapas:

a) $\forall j \in N, j$ é atingível a partir de 0 ,isto é,

$\exists P_{0 j}=\left(0, i_{1}, \ldots, i_{r}\right)$ um passeio entre 0 e $i_{r}=j$. Esse resultado é imediato pela construção do conjunto de nós $\mathbf{N}$. partir de y.

b) Para 0 grafo $G_{P G}$ tem que $\forall j \in N, 0$ nó $0 \in N$ é atingível a

Tomemos $\bar{\mu} \in \mathbb{N}$ tal que $\bar{\mu} * F_{i j} \in \mathbb{Z} \forall i \in\{1,2, \ldots, p\} \forall j \in\{1,2, \ldots, q\}$ e seja $\bar{x} \in \mathbb{Z}^{q} \bar{x}_{i}=\mu \forall i \in\{1,2 \ldots, q\}$. Para o nó y,considere $x_{y} \in \mathbb{Z}^{q}$ tal que, $F x_{y} \equiv y, e \quad \lambda \in \mathbb{N}$ o menor inteiro tal que $\lambda \bar{x} \geqq x_{y}(\lambda$ sempre existe pois $\bar{x} e x^{y}$ são elementos de $\mathbb{Z}^{q}$, não negativos).

Sendo $w=\lambda \bar{x}-x_{y}$ vem $w \geqq 0, w \in \mathbb{Z}^{q}$.

nós,

Assumindo $I(w)=\{1,2, \ldots, \delta\}$, construimos então uma sequência de

$$
\begin{gathered}
y, y+F^{1}, y+2 F^{1}, \ldots, y+w_{i} F^{1}, \ldots, \\
y+\sum_{k=1}^{s-1} w_{k} F^{k}+F^{1}, \ldots, y+\sum_{k=1}^{s-1} w_{k} F^{k}+w_{s} F^{1}
\end{gathered}
$$

Claramente,a sequência de nós fornece um passeio entre y e y $+\sum_{k=1}^{s} w_{k} F^{k} \cdot$ Mas $y+\sum_{k=1}^{s} w_{r} F^{k}=$

$$
\begin{aligned}
& y+\sum_{k=1}^{q} w_{r} F^{k} \equiv \\
& y+F w \equiv y \\
& +F\left(\lambda \bar{x}-x_{y}\right) \equiv \\
& y+\lambda F \bar{x} \equiv \\
& y-y=0,
\end{aligned}
$$

ou seja, determinamos um passeio entre y e 0 e segue a afirmação.De (a) e (b) segue que $\forall(i, j) \in N \times N, j$ pode ser atingido a partir de $i$ pois 0 e atingivel a partir de i e $j$ a partir de 0

Observe que a hipótese de estarmos trabalhando com $F \in \mathbf{Q}^{\text {qxp }}$ foi utilizada para que verificassemos que 0 é atingível a partir de qualquer 
nó.De fato,se tomarmos $F=[\sqrt{2}-1], \forall n \in \mathbb{N}, \mathrm{n}(\sqrt{2}-1) \not \equiv 0$ e portanto 0 näo é atingivel a partir de $\sqrt{2}-1$.

Um corolário importante é o seguinte:

\section{COROLÁRJO 5.2.11}

Para o grafo $G_{P G}$ tem que $f^{0} \in N$ se e somente se $f^{0}$ é atingivel a partir de 0 ,ou seja,se e somente se o problema de determinação de um passeio entre 0 e $f^{0}$ é viável.

\section{Demonstração}

\section{Imediata}

0 grafo $G_{P G}$ tendo sendo construido a partir do problema de grupo (PG mantém estreitas relaçōes com 0 mesmo.O processo construtivo do teorema anterior nos mostra que elementos $x \in \mathbb{Z}^{q}: F x \equiv y$ estão relacionados a passeios no grafo.Na verdade, valem alguns resultados ainda mais fortes, relacionain do elementos viaveis e passeios.Para prová-los consideremos a seguinte :

\section{DEFINIÇÃO 5.2.12}

Dado $y \in \mathbb{R}^{p}$ definimos o conjunto $X_{y}$ por

$$
X_{Y}=\left\{x \in Z^{q} \mid F x \equiv y \quad x \geqq 0\right\}
$$

Adotando esta definição temos

'PROPOSIÇÃO 5.2.13

A cada passeio $P_{i j}=\left(i_{1}, i_{2}, \ldots, i_{r}\right)$ entre $i_{1}=i e i_{r}=j \mathrm{em} G_{P G, \mathrm{e}}$ xiste associado $x \in X_{j-i}$ tal que $c\left(P_{i j}\right)=\left\langle d_{1} x\right\rangle$. 


\section{Demonstração}

Sendo $P_{i j}$ um passeio entre i e j, temos que

$\forall k \in\{1,2, \ldots .-1\} i_{k+1} \in \operatorname{suc}\left(i_{k}\right)$ e portanto,por construção de $N$, $i_{k+1}=F^{l_{k}}+i_{k}$ para algum $l_{k} \in\{1,2, \ldots, q\}$.

Assim, $j \equiv i_{r} \equiv \sum_{k=2}^{r-1} F_{k}^{l}+i$ e claramente $\sum_{k=2}^{r-1} F_{k}^{l} \equiv j-i$. Logo, $\exists \bar{x} \geqq 0$ inteiro tal que $F \bar{x} \equiv j-i$,ou seja, $\bar{x} \in X_{j-i}$.

Como, $c\left(P_{i j}\right)=\sum_{k=2}^{r} c_{i_{k-1}} l_{k}$ e $\forall k \in\{1,2, \ldots, r\} c_{i_{k-1} i_{k}}=d_{l_{k}}$ por construção da função custo, temos

$c\left(P_{i j}\right)=\sum_{k=2}^{r} d_{l_{k}}=d_{i_{i}}\left(l_{1}\right.$ tal que $\left.i+F^{l_{1}}=i_{2}\right)$ e portanto,
$c\left(P_{i j}\right)=\sum_{k=2}^{r} d_{l_{k}}=\langle d, \bar{x}\rangle$

A recíproca desse resultado é imediata.

\section{PROPOSIÇÃO 5.2.14}

A cada $x \in X_{i-j}, i \in N$ e $j \in N$, existe associado um passeio $P_{i j}$ entre i e j tal que $c\left(P_{i j}\right)=\langle d, x\rangle$

Demonstração

Trivial

'Segue deste resultado que

\section{COROLÁRIO 5.2.15}

Seja $h \in X^{0}$

Se $h \neq 0$ então o passeio associado a $h$ segundo a proposição anterior é um circuito.

\section{Demonstração}


Trivial

Além disso temos :

LEMA 5.2.16

$\forall(i, j) \in N \times N \quad X_{j-i} \neq \emptyset$.

Demonstração

Sendo o grafo fortemente conexo,existe um passeio entre $i$ e $j$ a partir do qual e possível determinar $x \in X_{i-j}$

No capítulo 1 pudemos relacionar caminhos a bases viáveis no problema do caminho mínimo e reciprocamente, bases a caminhos. As idéias fundamentais daquele caso podem ser facilmente extendidas para o problema de grupo:

\section{DEFINIÇÃO 5.2.17}

Dado $W \cap \mathbb{R}_{+}^{n}$, definimos subfecho, $W^{S}$ de $W$ como sendo $W^{S}=$ $\left\{x \in \mathbb{Z}^{n} \mid 0 \leqq x \leqq y\right.$ para algum $\left.y \in W\right\}$ $\mathrm{S}(\mathrm{x})$.

No caso particular em que $W=\{x\}$ denotaremos seu subfecho por

FATO 5.2.18

Sejam $y \in \mathbb{R}^{p}, x \in \mathbb{X}_{y}$ e

$H=X_{0}=\left\{h \in \mathbb{R}^{q} \mid F h \equiv 0 h \geq 0\right\}$

Então $S(x) \cap H=\{0\} \Leftrightarrow \forall x^{\prime} \in S(x) \backslash\{x\}, x^{\prime} \notin X_{y}$.

Demonstração

$\Rightarrow$ ) Suponhamos que $\exists \hat{x} \in S(x) \hat{x} \leq x: \hat{x} \in X_{y}$ 
Então $\exists h: \leq h \leqq x, h$ inteiro tal que $x=\hat{x}+h$. Mas assim, vem que $y \equiv F x \equiv F \hat{x}+F h \equiv y+F h$ e portanto $h \in H \cap S(x)$. Logo, $H \cap S(x)=$ $0 \Rightarrow \forall x^{\prime} \in S(x) \backslash\{x\} x^{\prime} \in X_{\mathbf{y}}$.

$\Leftarrow)$ Se $S(x) \cap H \neq\{0\}$ então $\exists h \in \mathbb{Z}^{q} 0 \leq h \leqq x$, tal que $F h \equiv 0$.

Claramente $x^{\prime}=x-h \in X_{y}$ e segue a afirmação

De certa forma o fato de um dado vetor $x \in \mathbb{Z}^{q}$ ser o "menor" elemento viável no conjunto,o caracteriza como elemento extremal,correspondendo a idéia de vértices de programação linear,pois pode-se verficar que $\forall y \in \mathbb{R}^{p} \forall \mathrm{x} \in \mathrm{X}_{\mathrm{y}} \mathrm{x}=\mathrm{x}_{\mathrm{e}}+\mathrm{h}$ com $x_{\mathrm{e}}$ um elemento extremal e $h \in X_{0}$. Sob uma outra ótica,como elementos $x \in X_{y}$ estão associados a. passeios no grafo,elementos extremais estão associados a caminhos

\section{PROPOSIÇÃO 5.2.19}

$\operatorname{Sejam}(i, j) \in N \times N, x \in X_{j}-i$ e $H=X_{0}{ }^{\prime}$

Se $S(x) \cap H=\{0\}$ entä́o o passeio associado a $\times$ segundo a proposição 5.2.15 e um caminho entre i $\mathrm{e}$.

\section{Demonstração}

Considere $P_{i j}$ o passeio entre i e j conforme aquela proposiçåo e suponha que $P_{i j}$ possua um circuito $C=\left(i_{1}, i_{2}, \ldots, i_{t}\right), i_{t}=i_{1}$.

Por construção, $\forall k \in\{1,2, \ldots, t-1\} i_{k+1} \in \operatorname{auc}\left(i_{k}\right)$ e claramente $\exists l_{k} \in\{1,2, \ldots, q\} \mid i_{k+1} \equiv i_{k}+F^{l_{k}}$.

Assim, $i_{1} \equiv i_{t} \equiv \sum_{k=1}^{t-1} F_{k}+i_{1}$, donde segue que $\sum_{k=1}^{t-1} F_{4} \equiv 0$, ou seja, $\exists w \geq 0 \mid F w \equiv 0$. E de facil verificação que $w \leqq x$ e portanto $w \in S(x)$ contrariando a hipótese original.

Logo, $P_{i j}$ näo possue circuito sendo portanto um caminho

Naturalmente seria interessante se pudessemos assegurar a validade de uma recíproca desse resultado no sentido de que, a caminhos no grafo que pudessemos garantir que $S(x) \cap H=\{0\}$ com $x$ o elemento associado aquele caminho.Isso entretanto näo ocorre conforme mostra o seguinte exemplo. 


\section{EXEMPLO 5.2.20}

Considere o seguinte problema :

$$
\begin{aligned}
& \min c_{1} x_{1}+c_{2} x_{2} \\
& \text { sa } 0.1 x_{1}+0.5 x_{2}=0.1 \\
& x_{j} \dot{0} 0 \text { inteiro. }
\end{aligned}
$$

Tomando-se os nós i $=0$ e j $=0.1$ no grafo $G_{P G}$ um caminho é dado por $P=(0,0.5,0.6,0.1)$, em

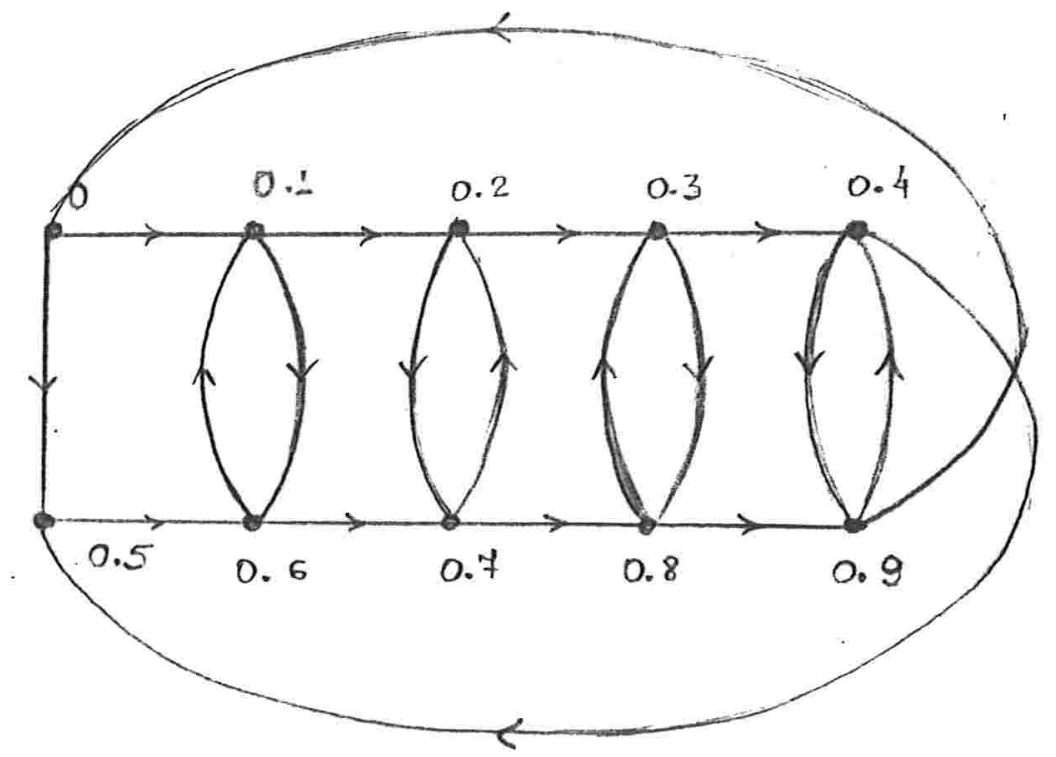

$\hat{x}=(0,2) \in S(x) \cap H$.

0 correspondente $x \in X_{0.1}$ é dado por $\mathrm{x}=(1,2)$ e claramente

Essa caracterização em termos de elementos extremais permite que se reproduza resultados clássicos de programação linear se fizermos analogias entre elementos extremais e vértices.

Para tanto utilizaremos a seguinte definição :

\section{DEFINIÇÃO 5.2.21}

Para o problema de grupos (PG), definimos a função perturbação $v($.$) , como$

$$
v: R^{p} \rightarrow \mathbf{R}
$$




$$
v(y)=\inf _{x \in X_{y}}<d, x>
$$

Primeiramente verifiquemos a seguinte propriedade :

\section{PROPOSIÇÃO 5.2.22}

Considere o problema de grupos (PG) e seja $(i, j) \in N \times N$ Então $v(j-i)=-\infty \Leftrightarrow \exists k \in\{1,2, \ldots, q\} \mid d_{k}<0$.

\section{Demonstração}

$\Rightarrow$ ) Imediata.

$\nLeftarrow)$ Seja $x \in X_{j-i}\left(X_{j-i} \neq \emptyset\right.$ do lema 5.2.16) e considere $\mu \in \mathbb{N}$ tal que $\mu F^{k} \equiv 0$.

Tomando $x(r)=x+r\left(\mu e^{k}\right), r \in N$, temos $F x(r) \equiv F\left(x+r\left(\mu e^{k}\right)\right) \equiv$ $F x+r\left(F\left(\mu e^{k}\right)\right) \equiv F x+r\left(\mu F^{k}\right) \equiv F x+r x 0 \equiv F x$ e portanto como $x(r) \geqq 0 x(r) \in X_{j-i}$.

Além disso

$\left.\lim _{r \rightarrow \infty}<d, x(r)\right\rangle=$

$\left.=\lim _{r \rightarrow \infty}<d, x+r \mu * e^{k}\right\rangle=$

$=<d, x\rangle+\lim _{r \rightarrow \infty} r * d_{j}=-\infty$

e portanto $v(j-i)=-\infty$

\section{COROLARIO 5.2.23}

Suponha que $f^{0} \in N$. Então

$v\left(f^{0}\right)=-\infty \Longleftrightarrow \exists k \in\{1, \ldots, ., q\} \mid d_{j}<0$

Demonstração

Trivial 
Estamos então em condiçōes de caracterizar soluçōes ótimas para os problemas de grupo.

\section{PROPOSIÇÃO 5.2.24}

Seja $(i, j) \in N x N, H=X_{0}$

$\{0\}$.

Se $v(j-i) \in \mathbb{R}$,então $\exists x^{*} \in \arg \min _{x \in X_{j-i}}\langle d, x>| S\left(x^{*}\right) \cap H=$

\section{Demonstração}

Para $\hat{x} \in \mathbb{Z}^{q}$ denotemos $S^{\prime}(\hat{x})$ ao conjunto de $S(\hat{x}) \backslash\{0\}$,isto é, $S^{\prime}(\hat{x})=\left\{x \in \mathbb{Z}^{q} \mid 0 \leq x \leqq \hat{x}\right\}$

Tome $x^{*} \in \arg \min _{x \in X_{j-i}}<d, x>$ e considere a seguinte construção

$x^{k}= \begin{cases}\mathrm{x}^{*} & k=0- \\ \mathrm{x}^{k-1}-w^{k} \text { para algum } w^{k} \in S^{\prime}\left(x^{k}-1\right) \cap H k \in \mathbb{N} & \mathrm{cc}\end{cases}$

E claro que $\exists k_{\max } \mid S^{\prime}\left(x^{k_{\max }+1}\right) \cap=\emptyset$ pois a sequência satisfaz $S^{\prime}\left(x^{*}\right) \subset S^{\prime}\left(x^{0}\right) \subset S^{\prime}\left(x^{1}\right) \ldots . . .0 m S^{\prime}\left(x^{k}\right) \neq S^{\prime}\left(x^{k+1}\right) \forall k \in\left\{0, \ldots, k_{\text {max }}\right\}$ e além disso $S^{\prime}\left(x^{*}\right)$ é finito.

Temos então

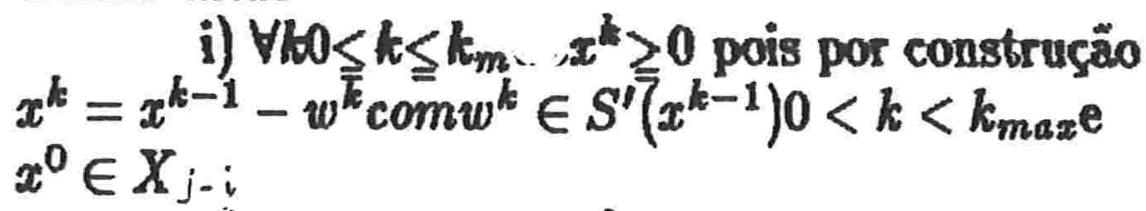

ii) $\forall k 0 \leqq k \leq k_{\max } x^{k} \in \mathbb{Z}^{q}$

Assim, $F x^{k} \equiv j-i$.

iii) $\forall k 0<\bar{k} \leqq k_{\max } F x^{k} \equiv F x^{k-1}-F w \equiv F x^{k-1}$

Como $v(j-i) \in \mathbb{R}$ temos $d \geqq 0$ donde $\forall h \in H,\langle d, h\rangle \geqq 0$.

Mas então temos

$\left\langle d, x^{k}\right\rangle=\left\langle d, x^{k-1}-w^{k}\right\rangle=\left\langle d, x^{k-1}-\left\langle d, w^{k}\right\rangle \leqq\left\langle d, x^{k-1}\right\rangle\right.$

e da otimalidade de $x^{0}=x^{*}$ segue que

$\left\langle d, x^{k}\right\rangle=<d, x^{*}>\forall k 0 \leq k \leq k_{\max }$

Em particular,$\left\langle d, x^{k_{\text {mas }}}\right\rangle=\left\langle d, x^{*}\right\rangle$ e como $x_{\text {max }}^{k} \in X_{j-i}$ vem que $x_{\text {mas }}^{k} \in \arg \min _{x \in X_{i-j}}\langle d, x\rangle$.

Por construçäo $S^{\prime}\left(x_{m a x}^{h}\right) \cap H=\emptyset$ e segue a afirmação 
Assim,verificamos que o problema,se possuir valor ótimo real,possue uma solução ótima "extremal". Observe que a demonstração corresponde a ideia de eliminarmos circuitos dos passeios associados aos elementos $x \in$ $X_{j-i}$.

Verifiquemos outras propriedades da função $v($.

\section{PROPOSIÇÃO 5.2.25}

é,

A função perturbação v(.) do problema de grupos é subaditiva,isto

$v\left(y_{1}, y_{2}\right) \in \mathbb{R}^{p} \times \mathbb{R}^{p} v\left(y_{1}+y_{2}\right) \leqq v\left(y_{1}\right)+v\left(y_{2}\right)$

Demonstração

trivial.

Seja $\left(y_{1}, y_{2}\right) \in \mathbb{R}^{\mathrm{p}} \times \mathbb{R}^{\mathrm{p}}$ Se $X_{y_{1}}=$ bou $X_{y_{2}}=\emptyset_{\text {a afirmação é }}$

Caso contrário, $X_{y_{1}} \neq \emptyset e X_{y_{3}} \neq \emptyset$ e temos

a) se $v\left(y_{1}\right) \in \mathbb{R} \operatorname{Rev}\left(\mathrm{y}_{2}\right) \in \mathbb{R}$ entäo

$\exists\left(x_{1}, x_{2}\right) \in X_{y_{1}} x X_{y_{3}}$ tais que

$v\left(y_{1}\right)=\left\langle d, x_{1}>\operatorname{ev}\left(y_{2}\right)=\left\langle d, x_{2}\right\rangle^{\prime}\right.$

Como $X_{y_{1}}+X_{y_{2}} \subset X_{y_{1}+y_{2}}$

segue $x_{1}+x_{2} \in X_{y_{1}+y_{2}}$ e claramente

$\left.v\left(y_{1}+y_{2}\right) \leqq<d, x_{1}+x_{2}\right\rangle=v\left(y_{1}\right)+v\left(y_{2}\right)$

elementos $X_{j_{i}}$ tal que

b) se $v\left(y_{1}\right)=-\infty$ então considere $\left\{x^{k}\right\}_{k \in \mathbb{N}}$ a sequência de

$\lim _{k \rightarrow \infty}\left\langle d, x^{k}\right\rangle=-\infty$

Seja $\hat{x} \in X_{y_{2}}$

$d, z^{k}>=-\infty$

Claramente $\forall k \in \mathbb{N}_{z}=\mathrm{x}^{\mathbf{k}}+\hat{\mathrm{x}} \in \mathbf{X}_{\mathrm{y}_{1}}+\mathbf{X}_{\mathrm{y}_{2}} \subset \mathbf{X}_{\mathrm{y}_{1}+\mathrm{y}_{2}}$ e $\lim _{k \rightarrow \infty}<$

* Por definiçáo dados $A \subset \mathbb{R}^{\mathrm{P} e B} \subset \mathbb{R}^{\mathrm{p}} A+B=\left\{y \in \mathbb{R}^{\mathrm{p}} \mid \exists(a, b) \in\right.$ $A x B: y=a+b\}$ 
c) Se $v\left(y_{2}\right)=-\infty$ a afirmação segue de forma analoga. $\operatorname{Logo}, v\left(y_{1}, y_{2}\right) \in \mathbb{R}^{\mathrm{P}} \mathbb{R}^{\mathrm{P}} \mathbf{v}\left(\mathrm{y}_{1}+\mathrm{y}_{2}\right) \leqq v\left(y_{1}\right)+v\left(y_{2}\right)$ segue a afirmaçãa

Nessas condiçōes temos

\section{TEOREMA 5.2.26}

Seja y $\in N$ e $x^{*} \in X_{y}$

$\mathrm{Se}\left\langle c, x^{*}\right\rangle=v(y)$ então $\forall x \in S\left(x^{*}\right)\langle c, x\rangle=v(F x)$

Demonstração

Seja y $\in S(x)$ e considere $\mathrm{h} \in \mathbb{Z}^{q} h \geqq 0$ tal que $x+h=x^{*}$.Temos $\langle c, x\rangle=v(y)=v\left(F x^{*}\right)=v(F x+F h)$

Mas $F x \in N e F h \in N$ e como v(.) e subaditiva,vem $\left\langle c, x^{*}\right\rangle=$ $v(F x+F h) \leqq v(f x)+v(F h)$

Como $\forall(x, h) \geq 0 v(f x) \leqq\langle c, x\rangle e v(F h) \leqq\langle c, h\rangle$, temos então $\left\langle c, x^{*}\right\rangle \leqq v(F x)+v(F h) \leqq v(F x)+\langle c, h\rangle \leqq\langle c, x\rangle+\langle c, h\rangle$

$=\left\langle c, x^{*}\right\rangle$

Segue que $v(F x)=\langle c, x\rangle$ e $v(F h)=\langle c, h\rangle$

\section{COROLÁRIO 5.2.27}

Nas condições do teorema anterior

$\forall j \in \operatorname{supp}\left\{x^{*}\right\}=\left\{j \in\{1, \ldots, q\} \mid x_{j}^{*}>0\right\}, v\left(F^{j}\right)=d_{j}$.

É importante salientar a semelhança entre o teorema 5.2.26 e o princípio de otimalidade que assegura que trechos de caminhos ótimos também são caminhos 6́timos.

Neste ponto estamos em condiçőes de verificar o seguinte resultado 
Para o problema de grupos (PG) e o correspondente grafo $G_{P G}$ vale $\forall(i, j) \in N \times N v(j-i)=k(i, j)$ definição 1.2.3.

onde $k(i, j)=\inf \{c(P) \mid P$ é um caminho entre $i$ e $j\}$ conforme

\section{Demonstração}

Segue das proposiçōes 5.2.13 e 5.2.14 que nos asseguram que, a cada passeio $P$ entre i e j existe $x \in X_{j-i}$ tal que $\langle d, x\rangle=c(P)(5.2 .13)$ e reciprocamente a cada $x \in X_{j-i}$ existe um passeio $P$ entre i e $\mathrm{j}$ tal que $c(P)=\langle d, x\rangle$

Segue trivialmente deste resultado que

\section{PROPOSIÇÃO 5.2.29}

O problema de grupos (PG) é equivalente ao problema de determinação de um caminho mínimo entre 0 e $f^{0} \mathrm{em} G_{P G}$.

\subsection{CONCLUSÃO}

Este capítulo tratou do problema de grupo (PG) procurando enfocar dois aspéctos : a relação entre ele e o problema de determinação da caminho mínimo em grafos ,e o estudo ainda que suscinto,da função perturbação v(.).

A relação entre (PG) e o problema definido sobre os grafos possibilita a utilização de algoritimos como Ford e $A^{*}$ para sua resolução.

Nesse sentido um algoritmo para a resolução de (PG) que merece destaque é conhecido como método de crescimento subaditivo (subadditive lifting) e nao pressupoe a existência do grafo $G_{P G}$.Para que o estudemos,alguns conceitos sobre a função $v($.$) aqui apresentados nos serão úteis$

Nosso próximo passo é o estudo de $A^{*}$ e do método de crescimento subaditivo para a resoluçảo de (PG) com a posterior verificação da relação entre eles. 
Capítulo 6

\section{A aplicação de $\mathbf{A}^{*}$ ao problema de grupos}

O capítulo anterior permitiu que caraterizassemos o problema de grupos como um problema de busca em grafos,o qual pode ser resolvido através de algoritmos de complexidade polinomial.

O objetivo central deste capítulo é a verificaçäo de que o grafo construido obedece as hipóteses basicas que permitem a utilização do algoritmo $A^{*}$ para a resolução de (PG), estabelecendo uma relação entre $A^{*}$ e o dual do problema de grupos que nos permite tratar $A^{*}$ como um algoritmo que gera funções viáveis em algum problema dual de (PG).

A razảo pela qual estudaremos o algoritmo $A^{*}$ para resolver o problema de grupos se deve não somente a sua medida de complexidade,mas também a sua relação com outros algoritmos para resolução do problema. 


\subsection{A VALIDADE DE $A^{*}$ PARA RESOLUÇÃO DE (PG)}

Conforme já estudamos se o grafo em questão for finito,conexo,sem laços e sem circuitos de custo negativo,estamos em condiçōes de aplicar o algoritmo $A^{*}$ que termina nesse caso em um número finito de passos.

Admitindo-se que o problema de grupos (PG) possua componentes racionais,sabemos que o grafo correspondente é finito;além disso,do processo construtivo,é também fortemente conexo de tal forma que precisamos apenas verificar que não possue laços nem circuitos de custos negativos.

Como o problema de grupos é ilimitado,se e só se

$$
\exists k \in\{1,2, \ldots, q\} \mid d_{k}<0,
$$

näo há perda de generalidade em assumirmos :

\section{HIPÓTESE 6.1.1}

Para o problema de grupos tem-se que

$$
\forall j \in\{1,2, \ldots, q\} \quad d_{j} \geq 0
$$

Para garantirmos a inexistência de laços assumiremos as hipóteses 5.2.7 e 5.2 .9 do capítulo anterior, quais sejam, a matriz de restriçōes do problema, bem como o vetor de termos independentes possuem coeficientes em $Q \cap\left[0,1\left[\right.\right.$ sendo que $\forall i \in\{1,2, \ldots p\} \forall j \in\{1,2, \ldots q\} \quad F^{i} \not \equiv \quad F^{j}$ e $F^{i} \not \equiv$.

Com essas considerações podemos estudar a utilização de $A^{*}$ na resolução de (PG).

\section{TEOREMA 6.1.2}

Suponhamos que $\exists\left\{h^{k}(.)\right\}_{k \in \mathbb{N}}$ uma sequência de subestimadores do caminho mínimo entre os nós 0 e $f^{0}$ em $G_{P G}$,que sejam admissiveis. è

Então sob a hipótese 6.1.1 o algoritmo $A^{*}$ aplicado ao grafo $G_{P G}$ termina em um número finito de passos quando se pode obter uma solução ótima do problema de grupos ou se detecta inviabilidade do mesmo. 
Sob as hipóteses assumidas, o grafo $G_{P G}$ é conexo,finito,e sem laços. Como o problema de determinaçã̃o de caminho mínimo em $G_{P G}$ não é ilimitado,estamos sob a hipótese do TEOREMA 1.1.14 e ao terminar o algoritmo detecta inviabilidade ou fornece um caminho mínimo $P^{*}$. Sendo os problemas equivalentes segue a afirmação.

No caso do problema de grupos temos que $\forall i \in N \quad k\left(i, f^{0}\right)=$ $v\left(f^{0}-i\right)$, e portanto, qualquer função $\mathrm{w}($.$) que subestime \mathrm{v}()$, pode ser utilizada para subestimar $k\left(i, f^{0}\right)$

\section{DEFINIÇÄO 6.1.3}

Chamamos de RELAXAÇÃO DE (PG) NO SENTIDO AMPLO,ou simplesmente,RELAXAÇÃO, à família de problemas

$P_{y}^{R}: \inf _{x \in X_{i}^{R}}\langle d, x\rangle$

onde,

$X_{y} \subset X_{y}^{\text {R }}$ com

$X_{y}=\left\{x \in \mathbb{Z}^{q} \mid F x \equiv y x \geqq 0\right\}$

Segue trivialmente desta definiçäo :

\section{PROPOSIÇÃO 6.1.4}

Sendo $v($.$) e v_{r}($.$) as funçōes :$

$v: \mathbb{R}^{p} \rightarrow \mathbb{R}$

e

$$
v(y)=\inf _{x \in X_{u}}<d, x>\text {. }
$$

respectivamente, vale :

$$
\begin{aligned}
& v_{r}: \mathbb{R}^{P} \rightarrow \mathbb{R} \\
& v_{r}(y)=\inf _{x \in X_{y}^{R}}\langle d, x>
\end{aligned}
$$

$\forall y \in \mathbb{R}^{p} \quad \mathbf{v}_{r}(y) \leqq v(y)$.

Demonstracão 
Capítulo 6: $A$ aplicação de $A^{*}$ ao problema de grupos

Trivial

Um exemplo de relaxação pode ser obtida através de um problema bastante simples de programação linear.

\section{DEFINIÇÃO 6.1.5} DE (PG) por:

Para o problema de grupos definimos a RELAXAÇÃO SIMPLES

onde

$$
\text { (RS) } \inf _{x \in X R S}\langle d, x>
$$

$$
\begin{aligned}
& X_{y}^{R S}=\left\{x \in \mathbb{R}^{q} \mid F x \geqq \hat{y} x \geqq 0\right\} \\
& \hat{y} \equiv y \quad \hat{y} \in\left[0,1\left[^{q}\right.\right.
\end{aligned}
$$

A verificação de que este problema é realmente uma relaxação de (PG) é bastante trivial.

\section{LEMA 6.1.6}

Para o problema de grupos (PG) e a relaxação simples temos que $\forall y \in \mathbb{R}^{\mathrm{q}} \quad \mathbf{X}_{\mathrm{y}} \subset \mathrm{X}_{\mathrm{y}}^{\mathrm{Rs}}$

Demonstração

Seja $y \in \mathbb{R}^{q}$

Se $X_{y}=$ nada a fazer.

Caso contrário seja $x \in X_{y}$. Como $F_{i j} \in[0,1[\cap \mathrm{D}, \forall i \in\{1,2, \ldots p\} \forall$ $\mathrm{j} \in\{1,2, \ldots . q\}$, e $x \in \mathbb{Z}_{+}^{q}$ é claro que Como i

$F x \in \mathbb{Q}$ e $F x \geqq 0$ temos $F x \geqq \hat{y}$.

Mas $y \equiv F x \equiv\lfloor F x\rfloor+\hat{y} \equiv \hat{y}$ e portanto, $\hat{y} \equiv y$.

Como $\quad x \geqq 0 x \in \mathbb{Z}^{q} e F x \geqq y$ segue que $x \in X_{y}^{R S}$

Dada uma relaxação qualquer,ela gera automaticamente subestimadores de caminho mínimo que Dodem ser utilizados dor $A^{*}$. 
Capítulo 6: $A$ aplicação de $A^{*}$ ao problema de grupos

\section{PROPOSIÇÃO 6.1.7}

Considere uma relaxação de (PG).Para o grafo $G_{P G}$ associado ao problema de grupos tem-se que $\hat{h}($.) definida por :

$\hat{h}: N \rightarrow \mathbb{R}$

com,

$$
\hat{h}(i)=v^{R}\left(f^{0}-i\right)
$$

$$
\begin{aligned}
& v^{R}: \mathbb{R}^{\mathrm{p}} \rightarrow \mathbb{R} \\
& v^{R}(y)=\inf _{x \in X_{y}^{R}}\langle d, x\rangle
\end{aligned}
$$

é subestimador admissivel do cáminho mínimo entre $i \in N$ e $f^{0} \in N$.

\section{Demonstração}

Da proposição 6.1.4 vem que

$$
\forall i \hat{h}(i)=v^{R}\left(f^{0}-i\right) \leq v\left(f^{0}-i\right)
$$

Como $v\left(f^{0}-i\right)=k\left(i, f^{0}\right)$ segue trivialmente a afirmação

Com este resultado temos que a relaxaçåo simples nos fornece subestimadores admissíveis. Como estamos interessados em obter $\hat{h}(i)=$ $v^{R}\left(f^{0}-i\right) i \in N$, podemos resolver a relaxação simples através de métodos como o simplex,obtendo $\hat{h}(0)=v^{R}\left(f^{0}\right)$ e a seguir determinando $\hat{h}(i)=$ $v^{R}(f-i)$ através de análise de sensibilidade.

Desde que possamos garantir que para uma relaxação,sua função perturbação $v^{R}($.$) é subaditiva, obtemos estimadores consistentes.$

\section{PROPOSIÇÃO 6.1.8}

Nas condições da proposição anterior,se a função $v^{R}($.$) é subadi-$ tiva, então $\hat{h}($.) é um subestimador consistente.

Demonstração 


$$
\begin{aligned}
& \hat{h}(i)=v^{R}\left(f^{0}-i\right)=v^{R}\left(f^{0}-j+j-i\right) \leqq v^{R}\left(f^{0}-j\right)+v^{R}(j-i) \\
& \hat{h}(i) \leqq v^{R}(j-i)+\hat{h}(j) \leqq v(j-i)+\hat{h}(j)=k(i, j) \\
& \text { ou seja, } \hat{h}(.) \text { é consistente }
\end{aligned}
$$

\section{COROLÁRIO 6.1.9}

Considere a relaxação simples de (PG)

$$
\begin{aligned}
\text { Então } \hat{h}: N & \rightarrow \mathbb{R} \\
\hat{h}(i) & =v^{R}\left(f^{0}-i\right)
\end{aligned}
$$
$f^{0} \in N$.

é um subestimador consistente do caminho mínimo entre $i \in N \mathrm{e}$

\section{Demonstração}

Segue da proposição anterior observando que a função perturbação da relaxação simples e subaditiva.*

Como estamos interessados em resolver o problema de grupos (PG), obtendo uma solução ótima do mesmo,ao resolver o problema definido sobre - grafo $G_{P G}$ devemos recuperar um caminho mínimo entre a origem $e$ o alvo (caso exista).

$\mathrm{Na}$ tentativa de melhorar o desempenho do algoritmo,podemos então associar aos nós alguma informação quanto ao elemento de $\mathbb{R}^{q}$ correspondente ao caminho entre a origem e o nó numa dada situação, Evitando assim, a "reconstituição" do caminho mínimo ao final do processo. Veja que assim podemos prescindir da utilizaçäo dos apontadores $a^{k}($.$) , cuja função$ básica, em termos operacionais,era a determinação da sequência de nós do caminho. o algoritmo

Para que essas consideraçōes fiquem mais claras vamos reescrever

* A demonstração deste resultado é analoga ao caso em que as restriçóes do problema são igualdades o que é verficado no apêndice 2. 
Capítulo 6: $A$ aplicação de $A^{*}$ ao problema de grupos

ALGORITMO 6.1.10

O ALGORITMO $A^{*}$ APLICADO A (PG)

$$
\begin{aligned}
& \llbracket g^{0}(0):=0 \\
& g^{0}(w):=+\infty ; \quad \forall w \notin\{0\} \\
& f^{0}(w):=g^{0}(w)+h^{0}(w) \quad \forall w \in N \\
& O^{0}=\{0\}_{i} \\
& C^{0}:=\emptyset \\
& k:=0 \text {; } \\
& x^{0}(w)=0 \quad \forall w \in N \\
& \text { Enquanto } O^{k} \neq \omega C^{k} \text { e } f^{0} \neq C^{k} \text { faça } \\
& \left\lceil\text { seja } w^{k} \in \arg \min _{w \in O^{k}}\left\{f^{k}(w)\right\}\right. \\
& F(k):=f^{k}\left(w^{k}\right) \\
& O^{k+1}:=O^{k} \backslash\left\{w^{k}\right\} \\
& C^{k+1}:=C^{k} \cup\left\{w^{k}\right\} \\
& \text { Para cada } j \in\{1,2, \ldots, q\} \text { faça } \\
& \left\lceil\operatorname{Seja} z \equiv w^{k}+F^{j} z \in\left[0,1\left[^{p}\right.\right.\right. \\
& \text { Se } g^{k}(z)>g^{k}\left(w^{k}\right)+d_{j} \\
& \text { entäo } \| g k+1(z):=g^{k}\left(w^{k}\right)+d_{j} \\
& x^{k+1}(z):=z^{k}\left(w^{k}\right)+w^{j} \\
& O^{k+1}:=O^{k} \cup\{z\} \\
& \text { Se } \left.z \in C^{z} \text { então } C^{k} \backslash\{z\}\right\rfloor \\
& \text { senäo }\left\lceil g^{k+1}(z):=g^{k}(z)\right. \\
& \left.x^{k+1}(z):=x^{k}(z)\right] \\
& f^{k+1}(z):=g^{k+1}(z)+h^{k+1}(z) \forall z \in N \\
& x^{k+1}(z):=x^{k}(z) \forall z \in N \backslash \operatorname{suc}\left(w^{k}\right) \\
& g^{k+1}(z):=g^{k}(z) \forall z \in N \backslash \operatorname{suc}\left(w^{k}\right) \\
& k:=k+1
\end{aligned}
$$

Sendo esse o próprio algoritmo $A^{*}$,nada há a se verificar quanto à sua validade.Outra rarão para o termos descrito é que segundo essa notação 
Capítulo 6: $A$ aplicação de $A^{*}$ ao problema de grupos

fica mais clara sua relação com outros algoritmos para a resolução do problema de grupos.

Alguns fatos interessantes se verificam nessas condiçốes :

\section{FATO 6.1.11}

Seja $k \in \mathbb{N}$ uma iteração qualquer do algoritmo $A^{*}$ aplicado a (PG).Entẵo

$$
\begin{aligned}
& \forall z \in C^{k} \cup O^{k} \\
& \exists x^{k}(z) \in \mathbb{R}^{q} \mid z=F x^{\mathbf{k}}(z) \text { e } g^{k}(z)=\left\langle d, x^{k}(z)>\right.
\end{aligned}
$$

Demonstração

Por indução sobre o número de iterações

$k=1$

Claramente $C^{1}=\{0\}$ e $O^{1}=\cup_{j=1}^{q}\left\{F^{j}\right\}$ e como

$x^{0}(0)=x^{1}(0)=0 x^{1}\left(F^{j}\right)=e^{j} \forall j \in\{1,2, \ldots, q\}$ segue que

$\left\langle d, x^{1}(0)\right\rangle=0=g^{1}(0) e\left\langle d, x^{1}\left(F^{j}\right)\right\rangle=d_{j}=g^{1}\left(F^{j}\right)$

\section{PASSO DA INDUÇÃO}

Suponhamos que $\forall k 1 \leqq k<p$ a afirmação seja verdadeira

$k=p$

Seja $z \in O^{k} \cup C^{k}$

Se $x^{k}(z)=x^{k-1}(z)$ e $g^{k}(z)=g^{k-1}(z)$ nada a fazer, a afirmação segue da hipótese de indução.

temos

Se $x^{k}(z) \neq x^{k-1}(z)$ (ou equivalentemente, $g^{k}(z) \neq g^{k-1}(z)$ ), entäo

$g^{k}(z)=g^{k-1}\left(w^{k}\right)+d_{j}$

$x^{k}(z)=x^{k-1}\left(w^{k}\right)+e^{j}$ para algum $\vec{j} \mid w^{k}+F \vec{j}=z$

$\mathrm{Da}$ hipótese de indução

$w^{k}=F x^{k-1}\left(w^{k}\right)$ e $g^{k-1}\left(w^{k}\right)=\left\langle d, x^{k-1}\left(w^{k}\right)\right\rangle$

e assim 
Capítulo 6: A aplicação de $A^{*}$ ao problema de grupos

$F^{j}=w^{k}+F^{j}=z$

$$
F x^{k}(z)=F\left(x^{k-1}\left(w^{k}\right)+e^{j}\right)=F\left(x^{k-1}\left(w^{k}\right)\right)+
$$

$$
\begin{aligned}
& g^{k}(z)=g^{k-1}\left(w^{k}\right)+d_{j}= \\
& =<d, x^{k-1}\left(w^{k}\right)>+d_{j}= \\
& =<d, x^{k-1}\left(w^{k}\right)+e^{j}>= \\
& =<d, x^{k}\left(w^{k}\right)>
\end{aligned}
$$

Do princípio de indução segue a afirmação

Verificamos com isto que 0 algoritmo constrói iterativamente pontos viáveis nos problemas $\inf _{x \in X_{y}}\langle d, x\rangle$, sendo que os rótulos $g^{k}(y)$ correspondem ao valor da função objetivo calculada neste ponto.Isso é equivalente a termos detectado um caminho de custo $g^{k}(y)$ entre 0 e y.

Ora, ao utilizarmos heurísticas consistentes,sabemos que a fechar um nó $j \in N$, determinamos o custo de um caminho mínimo entre a orígem e esse nó $\left(g^{k}(j)=k(0, j)\right)$ e portanto como $v(j)=k(0, j)$ é natural que nessas condiçōes,ao fechar um nó tenhamos obtido uma solução ótima de inf $f_{x \in X_{y}}\langle c, x\rangle$.

\section{LEMA 6.1.12}

Se o algoritmo $A^{*}$ aplicado a (PG) utiliza uma sequência $\left\{h^{k}(.)\right\}_{k \in \mathbb{N}}$ de subestimadores consistentes,então : $\forall z \in C^{k}, g^{k}(z)=v(z)=\left\langle d, x^{k}(z)\right\rangle$

Demonstração

Trivial,pois ao trabalharmos com estimadores consistentes

$$
\forall z \in C^{k} g^{k}(z)=k(0, z)=v(z)
$$


Nas condiçôes do lema anterior, temos que Se $\exists k^{*} \in N \mid f^{0} \in C^{k^{*}}$ então $x^{k^{*}}\left(f^{0}\right)$ é solução ótima de (PG).

\section{Demonstração}

Trivial

Veja que com isso ao utilizar a estimativa obtida a partir da relaxação simples do problema de grupo podemos afirmar que ao fechar o nó "alvo", teremos obtido a solução ótima do problema.É claro que o "bom desempenho" do algoritmo fica ainda assim comprometido,uma vez que é necessário resolvermos pelo menos,um problema linear para que determinemos $v^{R}\left(f^{0}\right)$ o que pode ter complexidade alta.

Como nos capítulos precedentes,estudemos a relação entre 0 algoritmo e o dual do problema.

POS

6.2 O ALGORITMO $A^{*}$ E O DUAL DO PROBLEMA DE GRU-

Como o grafo associado ao problema de grupo preserva as principais propriedades do problema original,é natural que alguma relaçäo entre os duais dos problemas de grupo e de caminho mínimo seja mantida.

O conceito de problema dual proposto por Tind e Wolsey [TW81], e que permite a eliminação de "gaps" entre valores ótimos primal e dual,no caso do problema do caminho mínimo se extende naturalmente ao caso de problemas definidos sobre grupos.

\section{DEFINIÇÃO 6.2.1}

POS a

Chamaremos de DUAL GERAL PARA O PROBLEMA DE GRU-

$$
\begin{gathered}
(\mathrm{DGPG}) \sup _{\psi(.) \in W_{\mathrm{g}}} \psi\left(f^{0}\right) \\
\text { onde, } \\
W_{g}=\left\{\psi(.) \in \mathcal{N} \mid \psi(F x) \leqq\langle d, x\rangle \forall x \in \mathbb{Z}^{q} x \geqq 0\right\}
\end{gathered}
$$


com

$$
\aleph \subset\left\{\psi: \mathbb{R}^{p} \rightarrow \mathbb{R}\right\}
$$

um caso particular deste problema é :

\section{DEFINIÇÄO 6.2.2}

Chamamos de DUAL SUBADITIVO PARA O PROBLEMA DE GRUPOS a

onde,

$$
(D P G S) \sup _{\psi(.) \in W_{* b}} \psi\left(f^{0}\right)
$$

$$
\begin{aligned}
& W_{\text {sub }}=\left\{w(.) \in \Im_{s u b} \mid \psi\left(F^{k}\right) \leq d_{k} \forall k \in\{1,2, \ldots, q\}\right\} \\
& \Im_{s u b}=\left\{\psi: \mathbb{R}^{\mathrm{P}} \rightarrow \mathbb{R} \mid \psi(.) \text { é subaditiva em } \mathbb{N}\right\} .
\end{aligned}
$$

A verificação de que o dual subaditivo é um caso particular do dual geral é basicamente a mesma que no caso da programaçảo linear inteira e será omitida. Uma outra definição de problema dual é a seguinte :

\section{DEFINIÇÄO 6.2.3}

Chamamos de DUAL ESTIMADOR PARA O PROBLEMA DE GRUPOS a :

$$
\begin{aligned}
& (\mathrm{DEPG}) \sup _{(.) \in W_{\text {sot }} \psi\left(f^{0}\right)} \\
& W_{\text {est }}=\left\{\psi(.) \in \Im \mid \psi(F x) \leqq v(j-i) \forall(i, j) \in N \times N \forall x \in X_{j-i}\right\} \\
& \Im=\left\{\psi: \mathbb{R}^{p} \rightarrow \mathbb{R}\right\}
\end{aligned}
$$

E de fácil verificação que este problema é também equivalente ao dual geral desde que o conjunto de definiçảo das funçōes seja o mesmo,isto $\hat{e}, k=3$.

A partir destas definições temos : 
Capítulo 6: $A$ aplicação de $A^{*}$ ao problema de grupos

\section{PROPOSIÇÃO 6.2.4.}

Nas condições das definições 6.2.2 e 6.2.3 tem-se :

i) $W_{\text {sub }} \subset W_{\text {est }}$

ii) $W_{\text {sub }}=W_{\text {eat }} \cap \Im_{\text {sub }}$

iii) $\sup _{\psi(.) \in W_{n \in b}} \psi\left(f^{0}\right)=\sup _{\psi(.) \in W_{\text {get }}} \psi\left(f^{0}\right)$

(DSPG) e (DEPG).

iv) $\exists \psi^{*} \in W_{\mathrm{sub}} \cap W_{\text {est }}$ tal que $\psi^{*}()^{\text {ent }}$ resolve

Demonstração

$\psi(y)=-\infty \quad \forall j$ é um elemento de $\left.W_{\text {sub }}\right)$

i) Seja $(i, j) \in N \times N$ \& $\phi(.) \in W_{\text {sub }}\left(W_{\text {sub }} \neq \emptyset\right.$ pois Tome $x^{*} \in \arg \min _{x \in X_{j-i}}\langle d, x\rangle\left(x^{*}\right.$ existe pois estamos trabalhando com custos não negativos), e seja $x \in X_{j-i}$,

$\psi(F x)=\psi(j-i)=\psi\left(F x^{*}\right)=\psi\left(\sum_{j=1}^{q} F^{j} x_{j}^{*}\right) \leqq \sum_{j=1}^{q} x_{j}^{*} \psi\left(F^{j}\right)$ $v(j-i)$

Como $\psi(.) \in W_{\text {sub }}, \psi(F x) \leqq \sum_{j=1}^{q} x_{j}^{*} \psi\left(F^{j}\right) \leqq \sum_{j=1}^{q} x_{j}^{*} c_{j}=\left\langle c, x^{*}\right\rangle=$

e portanto $\psi(.) \in W_{\text {est }}$

ii) É claro que $W_{\text {sub }} \subset W_{\text {sat }} \cap \Im_{\text {sub }}$

Seja então $\psi(.) \in W_{\text {est }} \cap \Im_{\text {sub }}$.

Como $\psi(.) \in W_{e a t} \psi\left(F^{k}\right) \leqq v\left(F^{k}\right) \leqq d_{k} \forall k \in\{1,2, \ldots, q\}$ e da subaditividade de $\psi($.$) segue a afirmaçẫo$

iii) Basta observar que

$$
\begin{aligned}
& \sup _{\psi \in W_{s u b}} \psi\left(f^{0}\right)=\sup _{\psi(.) \in W_{\text {eat }}} \psi\left(f^{0}\right) \\
& \text { iv) Imediato tomando-se } \phi^{*}=v(.)
\end{aligned}
$$

É imediato que

\section{TEOREMA 6.2.5}

Para o problema de grupos e os problemas duais subaditivo e estimadores vale o teorema forte de dualidade 
Demonstração

Trivial

A obtenção de funções viáveis no dual subaditivo é bem mais complexa que no caso do dual estimador. Apesar de que funçöes,como por exemploas lineares,sejam subaditivas elas, nem sempre atendem nossos objetivos. (Conforme veremos adiante,precisamos trabalhar,por exemplo,com funções que no conjunto de nós fechados por $\boldsymbol{A}^{*}$ se comporte como função valor ótimo de (PG)).

Procuremos relacionar o processo construtivo das funçöes $f^{k}($.$) uti-$ lizado por $A^{*}$ com dualidade,na mesma linha que no capítulo 4.

\section{PROPOSIÇÃO 6.2.6}

Seja $\hat{h}(.,$.$) uma função, \hat{h}: \mathbb{R}^{\mathrm{p}} \times \mathbb{R}^{\mathrm{p}} \rightarrow \mathbb{\mathbb { R }}$ satisfazendo

onde

$$
\hat{h}\left(y_{1}, y_{2}\right) \leqq k(i, j) \forall\left(y_{1}, y_{2}\right) \in N_{e}
$$

$$
N_{e}=\left\{\left(z_{1}, z_{2}\right) \in \mathbb{R}^{\mathrm{p}} \times \mathbb{R}^{\mathrm{p}} \mid \exists(\mathrm{i}, \mathrm{j}) \in \mathrm{N} \times N: j \equiv z_{2} e i \equiv z_{1}\right\}
$$

Então definindo-se $\psi($.) por

$\psi(y)= \begin{cases}\hat{h}(0, j) & \text { se } \exists j \in N \mid y \equiv j \\ -\infty & \text { cc. }\end{cases}$

tem-se que $\psi(.) \in W_{\text {est }}$

Demonstração

Trivial da definição de $W_{\text {eat }}$

Ao partir do algoritmo $A^{*}$ podemos também construir funçōes viáveis no dual estimador : 
Seja $\left\{z^{k}(., .)\right\}_{k \in \mathbb{N}}$ uma sequência de funções nas condições da proposição anterior,e suponhamos que $A^{*}$ utilize, na resolução do problema de grupo, uma sequência de estimadores $\left\{h^{k}(.)\right\}_{k \in \mathbb{N}}$ consistentes, $h^{k}\left(f^{0}\right) \equiv$ $0 \forall k$.

Então,a sequência $\left\{\psi^{k}(.)\right\}_{k \in \mathbb{N}}$ definida por

$$
\begin{gathered}
\psi^{k}: \mathbb{R}^{p} \rightarrow \mathbb{R} \\
\psi^{k}(y)= \begin{cases}\max _{l \in C^{k}}\left\{f^{k}(l)\right\} & \text { sey } \equiv f^{0} \\
z^{k}(0, j) & \text { se } y \equiv j \text { para algum } j \in N \mid\{f 0\} \\
-\infty & \text { c.c }\end{cases} \\
\text { satisfaz então } \psi^{k}(.) \in W_{\text {est }} \forall k \in \mathbb{N}
\end{gathered}
$$

Demonstração

Seja $k \in \mathbb{N}(i, j) \in \mathbb{N} \times N x \in X_{j-i}$

Se $F x \equiv j-i \equiv f^{0}$ então

$$
\psi^{k}(F x)=\psi^{k}\left(f^{0}\right)=\max _{l \in C^{k}}\left\{f^{k}(l)\right\}
$$

e da consistência dos estimadores

$$
\begin{aligned}
& \psi^{k}(P x)=\max _{l \in C^{k}}\left\{f^{k}(l)\right\} \leq \max _{l \in C^{k}}\left\{k\left(0, f^{0}\right)\right\}= \\
& =\max _{l \in C^{k}}\left\{v\left(f^{0}\right)\right\}=v\left(f^{0}\right)=v(j-i)
\end{aligned}
$$

Se $F x \equiv j-i \not \equiv \quad f^{0}$ então para algum $l \in N F x \equiv l$

e portanto

$\psi^{k}(F x)=\psi^{k}(l)=z^{k}(0, l) \leqq k(0, l)=v(l)=v(j-i)$

Assim, $\psi^{k} \in W_{\text {est }} \forall k \in \mathbb{N}$

A sequência de funçōes assim fechada goza da seguinte propriedade

\section{LEMA 6.2.8}

Nas condiçóes da proposição anterior, sendo $\left\{h^{k}(.)\right\}_{k \in \mathbb{N}}$ a sequência de subestimadores utilizadas por $A^{*}$ temos que :

$\mathrm{Se}$

$$
\begin{aligned}
& \text { i) } \forall(i, j) \in N \times N z^{k}(i, j) \leq z^{k+1}(i, j) \\
& \text { ii) } \forall j \in N\left\{h^{k}(j)\right\}_{k \in \mathbb{N}} \text { é não decrescente } \\
& \text { iii) } j \in N \text { é fechado na iteração } k \Rightarrow \forall k \geqq k^{\prime} \\
& h^{k}(j)=h^{k^{\prime}}(j)
\end{aligned}
$$


Capítulo 6: $A$ aplicação de $A^{*}$ ao problema de grupos

então $\forall y \in \mathbb{R}^{p}\left\{\psi^{\mathbf{k}}(y)\right\}_{\mathrm{k} \in \mathbb{N}}$ é não decrescente.

Demonstração

Sejam $k \in \mathbb{N}, y \in \mathbb{R}^{p}$

$$
\begin{aligned}
& \text { i) Se } y \not f^{0} y \equiv j \text { para algum } j \in N, \\
& \psi^{k}(y)=z^{k}(0, j) \leqq z^{k+1}(0, j)=\psi^{k+1}(y) \\
& \text { ii) Se } y \neq \equiv \quad f^{0} y \neq \equiv \quad j \forall j \in N \\
& \psi^{k}(y)=-\infty=\psi^{k+1}(y) \\
& \text { iii) Se } y \equiv f^{0} \text { entăo } \\
& \psi^{k}(y)=\max _{l \in C^{k}}\left\{f^{k}(C)\right\}
\end{aligned}
$$

Como a sequência $\left\{f^{l}\left(w_{l}\right)\right\}_{l \in \mathbb{N}}$ é não decrescente, $\left(w_{l}\right.$ o elemento escolhido para fechamento na iteração l) e os valores de $f^{l}\left(w_{l}\right)$ näo são alterados com as iteraçōes vem que

$$
\begin{aligned}
& \psi^{k}(y)=\max _{l \in C C^{k}}\left\{f^{k}(l)\right\}=\max _{l \in C^{k}}\left\{f^{k+1}(l)\right\} \\
& \leq \max _{l \in C C^{k+1}}\left\{f^{k+1}(l)\right\}=\psi^{k+1}(l) \\
& \overline{\bar{D}} \text { (i), (ii),e (iii) segue a afirmaçâo }
\end{aligned}
$$

Essa sequência de funçōes é limitada superiormente pela função valor ótimo,v(.) sendo não decrescente Portanto é de se esperar que "convirja" em algum sentido para $v($.$) , fornecendo uma solução ótima dual.$

\section{TEOREMA 6.2.9}

Nas condições da proposição 6.2.7,se $f^{0} \in N$ então na iteração $k^{*}$ na qual o algoritmo termina tem-se que $\psi^{k^{*}}($.$) é solução ótima do dual$ estimador

Demonstração

Como $\psi^{*} \in W_{\text {est }} \psi^{*}\left(f^{0}\right) \leqq v\left(f^{0}\right) \operatorname{mas}$ 
Capítulo 6: $A$ aplicação de $A^{*}$ ao problema de grupos

$v\left(f^{0}\right)$

$\psi^{k^{*}}\left(f^{0}\right)=\max _{l \in C k^{*}}\left\{g(l)+h^{k^{*}}(l)\right\} \geqq g\left(f^{0}\right)+h^{k^{*}}\left(f^{0}\right)=g\left(f^{0}\right)=$

Assim, $\phi^{k^{*}}\left(f^{0}\right)=v\left(f^{0}\right)$ e sendo viável resolve (DEPG)

É fácil obter exemplos que mostram que as funçōes $\psi^{k^{*}}$ assim construídas não são necessariamente subaditivas é possível obter funções subaditivas a partir da rotulação feita por $A^{*}$, quando são impostas condições mais fortes nas heurísticas. definiçăo:

Para que possamos construir tais funções utilizamos a seguinte

\section{DEFINIÇÄO 6.2.10}

Seja z(.) uma funcão definida nos nós do grafo $G_{P G}$, isto é,

$z: N \rightarrow \widetilde{\mathbb{R}}$. Definimos a extensäo de $z(). a \mathbb{R}^{p}$ como

$$
\begin{aligned}
& z_{e}: \mathbb{R}^{p} \rightarrow \mathbb{R} \\
& z_{e}= \begin{cases}z(j) & \text { se } y \equiv j \text { para algum } j \in \mathbb{N} \\
-\infty & \text { caso contrário }\end{cases}
\end{aligned}
$$

Doravante,utilizamos a seguinte notação simplificada

\section{NOTAÇÃO 6.2.11}

Para 0 algoritmo $A^{*}$ aplicado ao problema de grupos,ao utilizarmos as funçóes $f^{k}(),. g^{k}(),. h^{k}($.$) e g($.$) estaremos indicando suas extensões.$

\section{PROPOSIÇÃO 6.2.12}

Considere 0 algoritmo $A^{*}$ aplicado ao problema de grupos.Se a sequência $\left\{h^{k}(.)\right\}_{k \in \mathbb{N}}$ de subestimadores considerada satisfaz

$\forall k \in \mathbb{N}^{\mathbf{k}}\left(\mathrm{f}^{0}\right)=0, \mathrm{~h}^{\mathbf{k}}($.$) consistente e h^{k}($.$) subaditiva em \mathbf{N}$,entäo a sequência $\left\{\psi^{k}(.)\right\}_{k \in \mathbb{N}}$ definida por

$$
\phi^{k}: \mathbb{R}^{p} \rightarrow \mathbb{R}
$$


Capítulo 6: $A$ aplicação de $A^{*}$ ao problema de grupos

$$
\psi^{k}(y)=\left\{\begin{array}{l}
f^{k}(y) \\
f^{*}\left(j^{k}\right) c . c
\end{array} \quad \text { se } y \equiv j \text { para algum } j \in C^{k}\right.
$$

( $j_{k}$ o elemento escolhido para fechamento na iteração k) então

$$
\forall k \in \mathbb{N} \psi^{\mathrm{k}}(.) \text { é subaditiva }
$$

\section{Demonstração}

Sejam $\left(y_{1}, y_{2}\right) \in \mathbb{R}^{p} \times \mathbb{R}^{p}, \mathbf{k} \in \mathbb{N}$.Sem perda de generalidade $\left(y_{1}, y_{2}\right) \in\left[0,1\left[^{p} \times[0,1]^{p}\right.\right.$

i) Se $\left(y_{1}, y_{2}\right) \notin C^{k} \times C^{k}$ então

$\psi^{k}\left(y_{1}\right)+\psi^{k}\left(y_{2}\right) \geqq f^{k}\left(j_{k}\right) \geqq \psi^{k}\left(y_{1}+y_{2}\right)$ pois

$\psi^{k}(y) \geqq 0 \forall y$, e além disso, ou

$\psi^{k}\left(y_{1}\right)=f^{k}\left(j_{k}\right)$ ou $\psi^{k}\left(y_{2}\right)=f^{k}\left(y_{k}\right)$.

entäo,

ii) $\operatorname{Se}\left(y_{1}, y_{2}\right) \in C^{k} \times C^{k} e \exists j \in \mid\left(y_{1}+y_{2}\right) \equiv j$

$\psi^{k}\left(y_{1}+y_{2}\right)=f^{k}(y)=g(y)+h^{k}(y)=g\left(y_{1}+y_{2}\right)+h^{k}\left(y_{1}+y_{2}\right)=$

$=v\left(y_{1}+y_{2}\right)+{ }^{k}\left(y_{1}+y_{2}\right) \leqq v\left(y_{1}\right)+v\left(y_{2}\right)+h^{k}\left(y_{1}\right)+h^{k}\left(y_{2}\right)=$

$=\psi^{k}\left(y_{1}\right)+\psi^{k}\left(y_{2}\right)$

iii) $\operatorname{Se}\left(y_{1}, y_{2}\right) \in C^{k} \times C^{k}$ e $y_{1}+y_{2} \neq j \forall j \in C^{k}$

Como $y_{1} \in C^{k} e y_{2} \in C^{k}$ é claro que $\exists j \in N \mid j \equiv y_{1}+y_{2}$

Seja entäo $\hat{j} \in N \mid \hat{j} \in O^{k}, g^{k}(\hat{j}), \hat{j}$ um nó de algum caminho mínimo entre $0 \mathrm{ej}$.

Entäo

Mas $h^{k}($.$) é consistente e portanto$

$$
\psi^{k}\left(y_{1}+y_{2}\right)=f^{k}\left(j_{k}\right) \leqq g^{k}(\hat{j})+h^{k}(\hat{j})
$$

$\psi^{k}\left(y_{1}+y_{2}\right) \leqq g(\hat{j})+k(\hat{j}, j)+h^{k}(j)=g(j)+h^{k}(j)$

Assim, $\psi^{k}\left(y_{1}+y_{2}\right) \leqq g(j)+h^{k}(j)$

$=g\left(y_{1}+y_{2}\right)+h^{k}\left(y_{1}+y_{2}\right)=$

$=v\left(y_{1}+y_{2}\right)+h^{k}\left(y_{1}+y_{2}\right)$

$\leqq v\left(y_{1}\right)+v\left(y_{2}\right)+h^{k}\left(y_{1}\right)+h^{k}\left(y_{2}\right)=$

$=\psi^{k}\left(y_{1}\right)+\psi^{k}\left(y_{2}\right)$.

De (i),(ii),e (iii) segue a afirmação 
Capítulo 6: $A$ aplicação de $A^{*}$ ao problema de grupos

\section{COROLÁRIO 6.2.13}

Considere 0 algoritmo de Dijkstra aplicado ao problema de grupos, e seja $\left\{\psi^{k}(.)\right\}_{k \in \mathbb{N}}$ a sequência de funçōes construida conforme a proposição anterior,

$$
\forall k \in \mathbb{N} \psi^{\mathbf{k}}(.) \text { é subaditiva }
$$

\section{Demonstração}

Trivial, pois o algoritmo de Dijkstra corresponde a $A^{*}$ com heurísticas nulas, as quais são trivialmente consistentes e subaditivas

Esses resultados nos mostram alguns procedimentos que permitem a construção de funçōes viáveis em problemas duais de (PG) a partir das estimativas $f^{k}\left(\right.$.) de $A^{*}$. Uma "recíproca" desse resultado é válida no sentido de podermos construir subestimadores consistentes e admissíveis a partir de elementos viáveis nos problemas duais (DSPG) e (DEPG).

\section{PROPOSIÇÃO 6.2.14}

Seja $\psi(.) \in W_{\text {est }}$ e $\hat{h}($.$) definida por$

$$
\begin{aligned}
& h: N \rightarrow \mathbb{R} \\
& \hat{h}(j)=\psi\left(f^{0}-j\right)
\end{aligned}
$$

Então $\hat{h}($.$) é um subestimador admissível para o problema do cam-$ inho mínimo entre 0 e $f^{0}$

Demonstração

Trivial, pois

$$
h(j)=\psi(f 0-j) \leqq v\left(f^{0}-j\right)=k\left(j, f^{0}\right)
$$


Seja $\psi(.) \in W_{\text {sub }}$ Então

é um estimador consistente.

$$
\begin{aligned}
& \hat{h}: N \rightarrow \mathbb{R} \\
& \hat{h}(j)=\psi\left(f^{0}-j\right)
\end{aligned}
$$

Demonstração

Basta observar que qualquer $(\mathrm{i}, \mathrm{j}) \in N \times N$

$$
\begin{gathered}
\hat{h}(j)=\psi\left(f^{0}-j\right) \\
=\psi\left(f^{0}-i+i-j\right) \leqq \\
\psi\left(f^{0}-i\right)+\psi(i-j)=\hat{h}(i)+\psi(i-j) \\
\text { Mas } \psi(i-j) \leqq v(i-j)=k(j, i) \text { pois } \psi(.) \in W_{\text {sub }} \text { Logo, } \\
\hat{h}(j) \leqq k(j, i)+\hat{h}(i)
\end{gathered}
$$

Pudemos assim construir subestimadores consistentes e admissíveis para a utilização de $A^{*}$ na resolução do problema de grupos.Em particular, observe que a partir da relaxação simples do problema de grupo pudemos construir subestimadores consistentes (Corolário 6.1.9) o que ocorria pelo fato de que $v^{R}(.) \in W_{\text {sub }}$.

\subsection{CONCLUSÃO}

Além de verificar a viabilidade da aplicação de $A^{*}$ na resolução do problema de grupos, pudemos nesse capitulo, interpretá-lo como um algoritmo que gera iterativamente funções viáveis nos problemas duais de (PG). Observe que a escolha de nós para fechamento feita por $A^{*}$ utiliza adequadamente, "toda" a informação disponível sobre a "proximidade" do alvo, o que em geral não ocorre em outros algoritmos que resolvem (PG).

Nosso próximo passo seré o estudo de outro algoritmo para a resolução de (PG), conhecido como algoritmo de incrementos subaditivos, procurando relacioná-lo com o algoritmo $A^{*}$. 
Capítulo 7

\section{Algoritmos de incremento}

A utilização de conceitos de dualidade para a resolução do problema de grupos não se restringe a processos como $A^{*}$, que constroi implicitamente funçöes viáveis no problema de grupo.

Analogamente ao caso de programação linear, podemos construir iterativamente, uma sequência de funçōes viáveis no dual do problema, que mantenham, a cada passo, condiçōes de folgas complementares, procurando determinar viabilidade do problema original.Ao considerarmos uma sequência de funçöes viáveis no dual subaditivo do problema de grupo, que seja "não decrescente" com as iterações, obtemos um método bastante estudado na literatura, conhecido como MÉTODO DE INCREMENTO SUBADITIVO. Sequências de funções viáveis em outros problemas duais, como 
por exemplo o dual geral, também fornecem métodos aplicáveis a resolução do problema de grupo.

\section{1 - ALGORITMOS DE INCREMENTO}

Estaremos estudando o problema de grupos $(P G)$,

$$
\begin{aligned}
& \min _{x \in X_{G}}<d, x> \\
& X_{G}=\left\{x \in \mathbb{Z}^{q} \mid F x \equiv f^{0} x \geqq 0\right\}
\end{aligned}
$$

Para que possamos assegurar a existência de solução ótima do problema, vamos assumir a seguinte hipótese:

\section{HIPÓTESE 7.1.1}

O problema de grupos considerado satisfar
a.) $\forall i \in\{1,2, \ldots, p\} \forall j \in\{1,2, \ldots, q\}, F_{i j} \in \mathbb{Q} \cap[0,1]$ $f_{i}^{0} \in \mathbb{Q} \cap[0,1]$
b.) $\forall i \in\{1,2, \ldots, p\} \forall j \in\{1,2, \ldots, q\}, F^{j} \not \equiv 0 F^{j} \not \equiv F^{i}$
c.) $d \geqq 0$

Segundo Jonhson [Jo80A], o método de incrementos foi introduzido em 1973 em [Jo73a].

Repetindo idéias do algoritmo $\mathrm{A}, 0$ método gera iterativamente dois subconjuntos de elementos $e \in N$, correspondendo ao conceito de abertura e fechamento de nós.Gera-se também uma sequência de funçöes $\{\pi(.)\}_{k \in \mathbb{N}}$ satisfarendo

$$
\begin{aligned}
& \pi^{k}(e)=v(e) \forall \mathrm{e}^{n} \text { fechado" } \\
& \pi^{k}(e) \leqq\langle d, x\rangle \forall \mathrm{e}^{\text {"aberton }} \forall x \in X_{e}
\end{aligned}
$$

e de tal forma que a cada passo um elemento aberto possa ser incluido no conjunto de fechados (isto é, $\pi^{k}(e)=v(e)$ para algum e aberto).0 conjunto de abertos é então atualizado e o processo se repete até que se tenha obtido o valor ótimo do problema v $\left(f^{\circ}\right)$................ 
Em geral exige-se subaditividade das funções $\pi^{k}($.$) consideradas,$ quando entäo o método recebe a denominação "incremento subaditivo". Os trabalhos de Johnson usualmente apresentam o método subaditivo,sendo que as várias descriçốes apresentadas não possuem diferenças estruturais entre si.Em trabalho de 1974, Burdet e Johnson [BJ74] propóem uma particular construção de funçōes, sendo que uma extensão desse processo construtivo, aplicável a resolução de problemas de programação inteira, e elaborada em [BJ77].Em 1980, Johnson apresenta uma descrição geral do método [Jo80b], correlacionando-0 com outros métodos para a resolução de problemas inteiros.

Descrevendo-se o método de incremento subaditivo, temos:

MÉTODO 7.1.2

Método de Incrementos Subaditivos

INICLALIZAÇĀO

$$
\begin{aligned}
& C_{L}:=\{(0,0)\} ; \\
& O_{L}:=U_{j=1}^{q}\left\{\left(F^{j}, d_{j}\right)\right\} \\
& \text { Tome } \pi(.) \text { uma função subaditiva satisfazendo } \\
& \quad \pi(0)=0 \\
& \pi^{*}:=\pi\left(f^{j}\right)_{i}=d_{e} \forall\left(e, d_{e}\right) \in O_{L}
\end{aligned}
$$

\section{PASSO DE CRESCIMENTO}

$$
\begin{aligned}
& \text { Altere } \pi(.) \text { mantendo: } \\
& \text { i.) subaditividade } \\
& \text { ii.) } \pi(e) \leq d_{e} \forall\left(e, d_{e}\right) \in O_{L} \\
& \text { iii.) } \pi(e)=d_{e} \forall\left(e, d_{e}\right) \in C_{L} \\
& \text { iv.) } \pi\left(f^{0}-e\right)=\pi\left(f^{0}\right)-\pi(e) \forall\left(e, d_{e}\right) \in C_{L}
\end{aligned}
$$

e de tal forma que $\pi$ " tenha seu valor aumentado. 


\section{PASSO DE "ALCANÇE"}

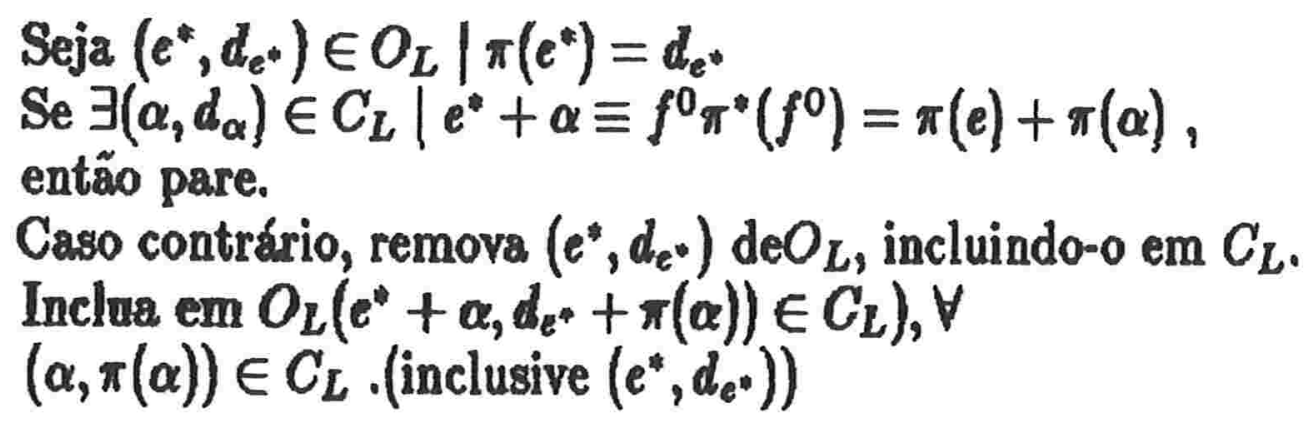

\section{FIM-MÉTODO}

Ao invés de verificarmos a validade do algoritmo na forma considerada, vamos fazer algumas pequenas alteraçöes no mesmo,que nos permitem trabalhar com uma notaçăo mais consistente com o que fizemos até então,sem que com isto se perca as características do algoritmo.

As condiçōes de (ii) e (iii) do passo de crescimento impöem que $\pi(e) \leqq d_{e} \forall\left(e, d_{e}\right) \in O_{L} \cup C_{L}$.

Consequentemente $\pi(e) \leqq \min _{d_{2}\left(t, d_{l}\right) \in Q_{L} \cup C_{2}}\left\{d_{l}\right\}=d^{*}$ e podemos incluir então em $O_{L}^{*}$ apenas os elementos para os quais $\left(e, d_{e}\right)=\left(e, d^{*}\right) \cdot$ Com isso näo é necessário que o conjunto $O_{L} e C_{L}$ correspondam a pares ordenados, bastando que se construa $\hat{g}($.$) ,uma função definida em \mathbb{R}^{p}, \hat{g}(e)=d^{*}$.

Vamos aqui procurar verificar a validade de um método de incrementos subaditivo,onde algumas restriçōes do conjunto de definição das funçōes $\left\{\pi^{k}(.)\right\}_{k \in \mathbb{N}}$ são relaxadas. $\operatorname{mos} \pi^{k}(.) \epsilon$

Ao invés de trabalharmos com funçôes $\pi^{k}($.$) subaditivas, considera-$

$$
W_{g}=\left\{\pi: \mathbb{R}^{p} \rightarrow \overline{\mathbb{R}} \mid \pi^{k}(\mathrm{Fx}) \leqq<\mathrm{d}, \mathrm{x}>\forall \mathrm{x} \geqq 0 \mathrm{x} \in \mathbb{Z}^{q}\right\}
$$

ou sejąfunções viáveis no dual geral do problema de grupo.Além disso, relaxaremos a restrição $\pi^{*}\left(f^{0}\right)=\pi\left(f^{0}-e\right)+\pi(e) e \in C_{L}$.

$\mathrm{Na}$ forma em que o método foi colocado não é possível assegurar que o mesmo termina,sendo para tanto necessário que se adote também outro critério de parada.

Nessas condiçōes temos 


\section{PASSO DE "ALCANÇE"}

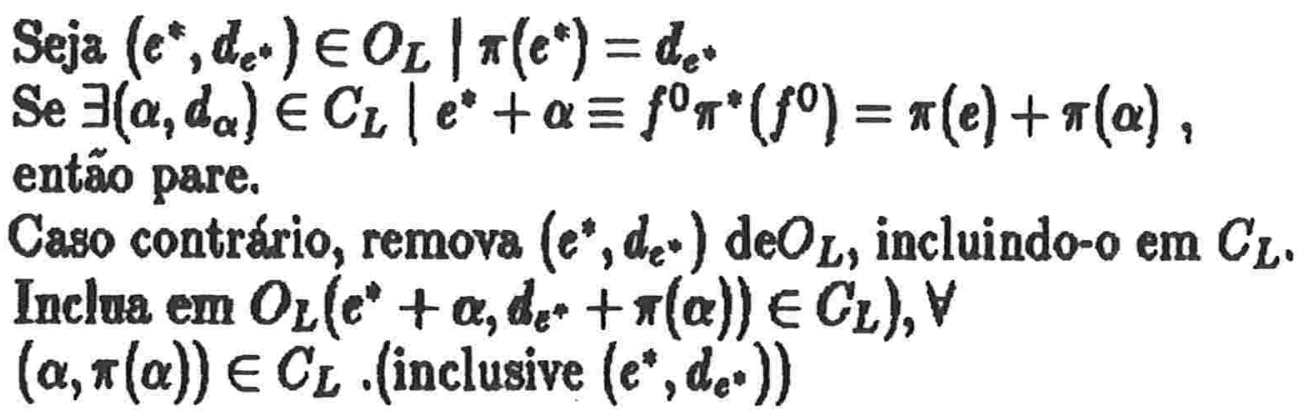

\section{FIM-MÉTODO}

Ao invés de verificarmos a validade do algoritmo na forma considerada, vamos fazer algumas pequenas alterações no mesmo,que nos permitem trabalhar com uma notaçăo mais consistente com o que fizemos até então,sem que com isto se perca as características do algoritmo.

As condiçöes de (ii) e (iii) do passo de crescimento impöem que $\pi(e) \leqq d_{e} \forall\left(e, d_{e}\right) \in O_{L} \cup C_{L}$.

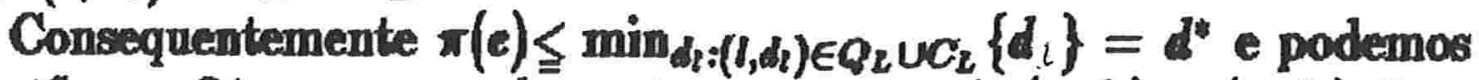
incluir entäo em $O_{L}^{*}$ apenas os elementos para os quais $\left(e, d_{e}\right)=\left(e, d^{*}\right) \cdot$.Com isso não é necessário que o conjunto $O_{L} C_{L}$ correspondam a pares ordenados, bastando que se construa $\hat{g}\left(\right.$.), uma função definida em $\mathbb{R}^{\mathrm{p}}, \hat{g}(e)=d^{*}$.

Vamos aqui procurar verificar a validade de um método de incrementos subaditivo,onde algumas restriçöes do conjunto de definição das funçöes $\left\{\pi^{k}(.)\right\}_{k \in \mathbb{N}}$ são relaxadas. $\operatorname{mos} \pi^{k}(.) \in$

Ao invés de trabalharmos com funçōes $\pi^{k}($.$) subaditivas,considera-$

$$
W_{g}=\left\{\pi: \mathbb{R}^{p} \rightarrow \overline{\mathbb{R}} \mid \pi^{\mathbf{k}}(\mathbb{F x}) \leqq<d, x>\forall x \geqq 0 x \in \mathbb{R}^{q}\right\}
$$

ou seja,funçóes viáveis no dual geral do problema de grupo.Além disso, relaxaremos a restrição $\pi^{*}\left(f^{0}\right)=\pi\left(f^{0}-e\right)+\pi(e) e \in C_{L}$.

$\mathrm{Na}$ forma em que o método foi colocado não é possível assegurar que o mesmo termina,sendo para tanto necessário que se adote também outro critério de parada.

Nessas condiçőes temos 
O Algoritmo de Incremento Relaxado

$$
\begin{aligned}
& \left\lceil\mathrm{g}^{0}(0):=0\right. \\
& g^{0}\left(F^{j}\right):=d_{j} ; j \in\{1,2, \ldots, q\} \\
& C_{f}^{0}:=\{0\} \text {; } \\
& O_{L}^{0}:=\cup_{j=1}^{q}\left\{F^{j}\right\} \text {; } \\
& g^{0}(y):=\infty \quad \forall y \in \mathbb{R}^{p} \backslash\left(O_{\mathrm{L}}^{\rho} \cup C_{\mathrm{L}}^{0}\right) ; \\
& \pi^{0}(.):=0 \\
& k:=0 \\
& \Omega^{1}=\left\{\pi(.) \in W_{G} \mid \pi\left(F^{j}\right) \leqq d_{j} j \in\{1,2, \ldots, q\}\right. \\
& \pi(0)=0 \\
& \pi\left(f^{0}\right) \geqq 0 \\
& \left.\exists j: \pi\left(F^{j}\right)=d_{j}\right\} \\
& \left.\forall\left(y_{1}, y_{2}\right) \in C_{L}^{k} \pi^{k}\left(f^{0}\right) \neq \pi^{k}\left(y_{1}\right)+\pi^{k}\left(y_{2}\right)\right)
\end{aligned}
$$

faça 【

Sejam $\pi^{k+1}(.) \in \Omega^{k+1} e j_{k} \in O^{k} \mid \pi^{k+1}\left(j_{k}\right)=g^{k}\left(j_{k}\right)$

onde $\Omega^{k+1}=\left\{\pi(.) \in W_{G} \mid\right.$

$$
\begin{aligned}
& \pi(e) \leqq g^{k}(e) \forall e \in O_{L}^{k} \\
& \pi(e)=g^{k}(e) \forall e \in C_{L}^{k} \\
& \pi\left(f^{0}\right) \geq \pi^{k}\left(f^{0}\right)
\end{aligned}
$$$$
\left.\exists \hat{e} \in O_{L}^{k} \backslash C_{L}^{k}: \quad \pi(\hat{e})=g^{k}(e)\right\}
$$

$O_{L}^{k+1}:=O_{L}^{k} \backslash\left\{j_{k}\right\} ;$

$C_{L}^{k+1}:=C_{L}^{k} \cup\left\{j_{k}\right\}$;

$g^{k+1}(y):=g^{k}(y) \quad \forall y \in \mathbb{R}^{p}$

Para cada $e \in C^{k+1} \backslash\{0\}$ faça

$$
\begin{aligned}
& \left\lceil\text { Seja } y \equiv j_{k}+\mathrm{e} y \in[0,1[p\right. \\
& \text { Se } g^{k}(y)>g^{k}\left(j_{k}\right)+g^{k}(e) \\
& \text { então } \\
& k:=k+1 \\
& \begin{array}{l}
g^{k+1}(y):=g^{k}(j k)+g^{k}(e) \\
\left.O_{L}^{k+1}:=O_{L}^{k} \cup y ;\right\rfloor \\
\Downarrow
\end{array}
\end{aligned}
$$

Colocado nesta forma o algoritmo se assemelha muito ao algoritmo $A^{*}$ aplicado a (PG),quanto ao processo construtivo dos conjuntos $C_{t}^{k}$. $O_{I}^{k}$ 
e dos rótulos $g^{k}($.$) . Assim como no caso do algoritmo A^{*}$, existe uma relação entre os valores $g^{k}(e)$ e $e \in C_{L}^{k}$ e as soluções de $\min _{x \in X_{e}}\langle d, x\rangle$.

\section{PROPOSIÇÃO 7.1.4}

Sejam $k \in \mathbb{N}$ e e $\in C_{L}^{k} \cup O_{L}^{k}$

Então $\exists x \in X_{e} \mid g^{k}(e)=\left\langle d_{3} x\right\rangle^{*}$

\section{Demonstração}

Analoga à demonstração do fato 6.1.11,lembrando-se apenas que estamos trabalhando com um critério de abertura de elementos distinto de $A^{*}$.

Outros resultados válidos para $A^{*}$ também se verificam

\section{LEMA 7.1.5}

Seja $k \in \mathbb{N}, e \in \mathbb{R}^{p}$

Se $g^{k}(e) \neq g^{k+1}(e)$ então $\exists x \in X_{e}:\left\langle d, x>=g^{k+1}(e)<g^{k}(e)\right.$.

Demonstração

Do processo de atualizaçåo de $g^{k}(),. \forall i \in 0_{l}^{k} \cup C_{l}^{k} g^{k+1}(e) \neq g^{k}(e)$ se e só se ${ }^{h+1}(e)<q^{h}(e)$

Logo, pelo teorema anterior segue a afirmação

COROLÁRIO 7.1.6

Seja $k \in \mathbb{N}, e \in O_{L}^{k} \cup C_{L}^{k}$

Se $g^{k}(e)=v(e)$ então $\forall k^{1} \geqq k g^{k^{1}}(e)=v(e)$.

* Como nos capitulos anteriores $X_{e}=\left\{x \in \mathbb{Z}^{9} \mid F x \equiv e x \geqq 0\right\}$ 
Demonstração

Imediato pelo lema anterior

Săo imediatos também os seguintes fatos

FATO 7.1.7

$\forall k \in \mathbb{N C}_{\mathrm{L}}^{\mathrm{k}} \cup \mathrm{O}_{\mathrm{L}}^{\mathrm{k}} \subset \mathrm{C}_{\mathrm{L}}^{\mathrm{k}+1} \cup \mathrm{O}_{\mathrm{L}}^{\mathrm{k}+1} \subset \mathrm{N}$

$\left(N=\left\{y \in\left[0,1|p| \exists x \in \mathbb{Z}^{a} x \geqq 0: y \equiv F x\right\}\right)\right.$

Demonstração

Trivial

EATO 7.1.8

$\forall k \in \mathbb{N} \#\left(C_{\mathrm{L}}^{k} \cup 0_{\mathrm{L}}^{k}\right) \leqq \# \mathbb{N}<\infty$

Demonstração

Trivial

Esses resultados derivam apenas do processo construtivo dos conjuntos $O_{L}^{k}$ e $C_{L}^{k}$ e de $g^{k}($.$) , assim como o algoritmo A^{*}$, e independem da sequência $\left\{\pi^{k}(.)\right\}_{k \in \mathbb{N}}$ utilizada.

As funçōes $\left\{\pi^{k}(.)\right\}_{b \in \mathbb{N}}$ que estamos considerando são viáveis no dual geral do problema de grupos. Como o conceito de viabilidade dual independe do vetor de termos independentes, $f^{0}$, temos que os duais dos problemas $P e: \min _{\mathfrak{x} \in X_{0}}\langle d, x>$ possuem o mesmo conjunto de pontos viáveis, $W=\left\{\psi: \mathbb{R}^{P} \rightarrow \overline{\mathbb{R}} \mid \psi(\mathrm{Px}) \leqq\left\langle d, x>\forall x \geqq 0 x \in \mathbb{Z}^{q}\right\}\right.$. 
Assim, se $\pi^{k}(e)=\langle d, x\rangle$ para algum $x \in X_{e}$ então,pelo teorema fraco de dualidade, $\pi^{k}(e)=\min _{x \in X_{e}}\left\langle d_{1} x\right\rangle$, ou seja, determinamos 0 valor ótimo de $P_{e}$.

Assim,

\section{TEOREMA 7.1.9}

Seja $k \in \mathbb{N}, \pi(.) \in \Omega^{\mathbf{k}}$ e $\bar{e} \in O^{k} \cup C^{k}$

Se $\pi(\bar{e})=g^{k}(\bar{e})$ então $\pi(\bar{e})=v(\bar{e})=\inf _{x \in X_{\varepsilon}}\langle d, x\rangle$

\section{Demonstração}

Da proposição 7.1.4 $\exists x \in X_{\bar{\varepsilon}} \mid g^{k}(\bar{e})=\langle d, x\rangle$

e portanto $g^{k}(\bar{e}) \geq v(\bar{e})$ mas $\pi(.) \in \Omega^{k} \subset W_{G}$, e dal,

$g^{k}(\bar{e})=\pi(\bar{e})=\pi(F x) \leqq v(F x)=v(\bar{e})$

Segue então que $g^{k}(\bar{e})=v(\bar{e})$

Decorre deste teorema que :

\section{TEOREMA 7.1.10}

Seja $k \in \mathbb{N}$ e $\pi \in \Omega^{k}$. Então $\forall l \in C^{k} \pi(e)=g^{k}(e)=v(e)$

Demonstracão

Basta observar que $\forall e \in C^{k} \pi(e)=g^{k}(e)$ por construção.Do teorema anterior segue a afirmação.

Consequentemente 
Seja $\left\{\pi^{k}(.)\right\}_{k \in \mathbb{N}}$ uma sequência de funções, $\pi^{k} \in \Omega^{k} \forall k \in \mathbb{N}$ e $e \in C^{k}$ para algum $k \in \mathbb{N}$.Entäo $\forall k \geqq k g^{k}(e)=v(e)$

\section{Demonstração}

Pelo teorema anterior, $e \in C^{\bar{k}} \Rightarrow g^{\bar{K}}(e)=v(e)$ Logo,pelo corolário 7.1.6 segue a afirmaçäo

Veja que resultados semelhantes a esses eram obtidos quando,no algoritmo $A^{*}$, utilizavamos subestimadores consistentes,pois naquele caso, ao fecharmos um nó $e \in N$ tinhamos detectado o valor ótimo do problema de $P_{e}$.

Para 0 algoritmo de incrementos vale ainda :

\section{PROPOSIÇÃO 7.1.12}

Seja $\left\{\bar{n}^{k}(.)\right\}_{k \in \mathbb{N}}$ tal que $\overline{\bar{n}}^{k}(.) \in \Omega^{k} \forall k \in \mathbb{N}$ e $e \in N$ Então, $\forall k \in \mathbb{N}$

ou

$$
e \in C^{k} e g^{k}(e)=v(e)
$$

ou

$$
\begin{gathered}
\forall x^{*} \in \arg \min _{x \in X_{k}}<d, x>\exists x \in S\left(x^{*}\right): \\
F x \in O_{L}^{k} \backslash C_{L}^{k} \text { e } g^{k}(F x)=v(F x)
\end{gathered}
$$

Demonstração

Seja $e \in O_{L}^{k} \backslash C_{L}^{k}$

Se $e \in C_{L}^{k}$, nada a faser

Caso contrário, seja $x^{*} \in \arg \min _{x \in X_{e}}\langle d, x\rangle$ (tal elemento existe pois $X_{e} \neq(e d \geqq 0$ ) 
Se $v\left(F x^{k}\right)=v(e)=g^{k}(e)$ a afirmação é trivial.

Senäo ,considere $S\left(x^{*}\right)$ o subfecho de $x^{*}$ (conforme definição 5.2.17) e seja $\Lambda=\left\{x \in S\left(x^{*}\right) \mid F x \in C_{L}^{k}\right\}$.

Claramente $\Lambda \neq \emptyset(0 \in \Lambda)$ e finito, e portanto $\exists x_{m a x} \in \Lambda$ tal que $\forall x \in \Lambda x \geq x_{\max }$

Como $x_{\max } \neq x^{*}$ (pois caso contrário $g^{k}\left(F x^{*}\right)=g^{k}(e)=v\left(F x^{*}\right)=$ $v(e))$ temos que $\exists \bar{j} \in\{1,2, \ldots, q\} \mid x_{\max }+e^{j} \leqq x^{*}$.

*

Sendo $x^{*} \in \arg \min _{x \in X_{0}}<d, x>$ e $x_{j}^{*}>0$, segue que $v\left(F^{j}\right)=d_{j}$

Temos dois casos a considerar

i) Se $F^{\vec{j}} \in 0_{L}^{k} \backslash C_{L}^{k}$ então $g^{k}\left(F^{j}\right)=d_{j}=v\left(F^{j}\right)$ pois $g^{1}\left(F^{j}\right)=d_{j} \mathrm{e}$ esse rótulo não é maìs alterado.

ii)Se $F^{j} \in C_{L}^{k}$,então na primeira iteração, $k^{1}$, na qual $F^{j} \in C_{L}^{k^{1}}$ e $F x_{\text {max }} \in C_{L}^{k^{1}}$, temos $y=F x_{\text {max }}+F^{j} \in O_{L}^{h^{1}}$ e $g^{k 1}(y)=v\left(F x_{\text {max }}\right)+v\left(F^{j}\right)=$ $v(y)$,não sendo esse o rótulo alterado.

Como por construção $x_{\max }+e^{j} \notin \Lambda$ segue a afirmaçäo

Caso nosso critério de parada do método fosse apenas $O_{L}^{k} \backslash C_{L}^{k}=\emptyset$ teríamos mostrado que o método não termina enquanto não determinar 0 valor ótimo de cada problema $\min _{x \in X_{e}}\langle d, x\rangle$ para cada $e \in \mathbb{R}^{p} \mid X_{e} \neq \emptyset$.

Como estamos interessados em obter o valor ótimo do problema de grupos precisamos mostrar que mesmo com o critério de parada utilizado - método não termina enquanto não determinar ou inviabilidade ou uma solução ótima (PG).

\section{TEOREMA 7.1.13}

Sob a hipótese 7.1.1 0 algoritmo de incremento relaxado aplicado a (PG) termina ao final de um número finito de $k^{*}$ de iteraçōes,quando então

* Veja que se $v\left(F^{j}\right)<d_{j},<d_{3} x^{*}>=v\left(F x^{*}\right) \leqq \sum_{j=1}^{q} x_{j}^{*} v\left(F^{j}\right)<$ $\sum_{j=1}^{q} x_{j}^{*} d_{j}=<d, x^{*}>$ absurdo 
ou $O_{L}^{k^{*}} \backslash C_{L}^{k^{*}}=\emptyset$ e o problema é inviável,

$$
\begin{aligned}
& \text { ou } \exists\left(e_{1}, e_{2}\right) \in C_{L}^{k^{*}} x C_{L}^{k^{*}} \mid \pi^{k^{*}}\left(e_{1}\right)=\pi^{k^{*}}\left(f^{0}\right)-\pi^{k^{*}}\left(e_{2}\right) f^{0} \equiv e_{1}+ \\
& e_{2}, e \pi^{k^{*}}\left(f^{0}\right)=v\left(f^{0}\right) \\
& \text { ou } \Omega^{k^{*}}=\emptyset
\end{aligned}
$$

\section{Demonstração}

Como $\forall k \in \mathbb{N} C_{L}^{k} \cup O_{L}^{k}$ é finito,segue que ao final de um número finito $k^{*}$ de iterações ou $O_{L}^{k^{*}} \backslash C_{L^{k}}^{k^{*}}=\emptyset$ ou $\exists\left(e_{1}, e_{2}\right) \in C_{L}^{k^{*}} x C_{L}^{k^{*}} \mid \pi^{k^{*}}\left(f^{0}\right)-\pi^{k^{*}}\left(e_{2}\right)=\pi \pi^{k^{*}}\left(e_{1}\right)$ com $f^{0}=e_{1}+e_{2}$ ou $\Omega^{k}=\emptyset$, e portanto, o algoritmo termina.

Temos dois casos a estudar :

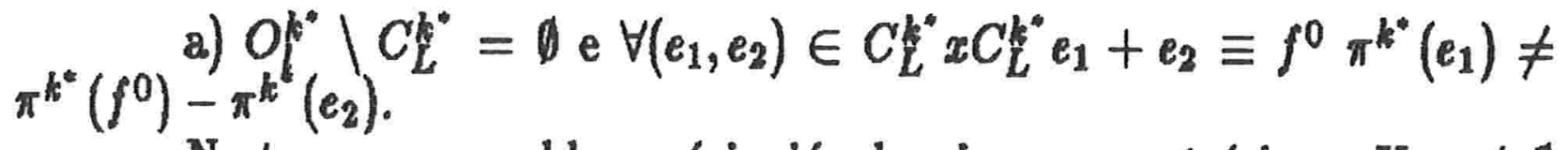

Neste caso o problema é inviável pois,caso contrário,se $X_{f 0} \neq 0$ sabemos que $\forall x^{*} \in \arg \min _{x \in X_{s}}<d, x>\exists x \in S\left(x^{*}\right) \mid F x \in O_{L}^{k^{*}} \backslash C_{L}^{k^{*}}$

b) $\exists\left(e_{1}, e_{2}\right) \in C_{L}^{k^{*}} x C_{L}^{k^{*}} \mid \pi^{k^{*}}\left(e_{1}\right)=\pi^{k^{*}}\left(f^{0}\right)-\pi^{k^{*}}\left(e_{2}\right) e_{1}+e_{2} \equiv f^{0}$ Sejam $\left(x_{1}, x_{2}\right) \in X_{t_{1}} x X_{e_{2}} \mid g^{k^{*}}\left(e_{1}\right)=\left\langle d, x_{1}\right\rangle g^{k^{*}}\left(e_{2}\right)=\left\langle d, x_{2}\right\rangle$.

Claramente $\bar{x}=x_{1}+x_{2} \in X_{f 0} \quad \pi^{k^{*}}\left(e_{1}\right)+\pi^{k^{*}}\left(e_{2}\right)=g^{k^{*}}\left(e_{1}\right)+$ $g^{k^{*}}\left(e_{2}\right)=\left\langle d, x_{1}+x_{2}\right\rangle=\left\langle d, \bar{x}>\right.$ Como $\pi^{k^{*}}(.) \in \Omega^{k^{*}} e \pi^{k^{*}}\left(f^{0}\right)=\langle d, \bar{x}\rangle$, segue que $\pi^{k^{*}}\left(f^{0}\right)=v\left(f^{0}\right)$.

De (a) e (b) segue a afirmação.

Desde que possamos asseguar a existência de uma sequência de funções $\left\{\pi^{k}(.)\right\}_{k \in \mathbb{N}}$ satisfazendo $\pi^{k}(.) \in \Omega^{k} \forall k \in \mathbb{N}$, o teorema 7.1.13 nos garante que o algoritmo termina em um número finito de passos, detectando a inviabilidade ou uma solução ótima do problema.Assim,verifiquemos que tal sequência pode ser sempre construida. 
Para o método de incrementos sempre existe uma sequência de funçōes $\left\{\pi^{k}(.)\right\}_{k \in \mathbb{N}}$ onde $\pi^{k}(.) \in \Omega^{k} \forall k \in \mathbb{N}$.

\section{Demonstração}

Tomando-se a sequência de funções invariantes com as iteraçōes, $\pi^{k}()=.v(.) \forall k \in \mathbb{N}$,segue a afirmação pela proposição 7.1.12 observando-se que

$$
\forall k \in \mathbb{N} \exists e \in \mathrm{O}_{\mathrm{L}}^{K} \backslash \mathrm{C}_{\mathrm{L}}^{\mathrm{k}} \mathrm{g}(\mathrm{e})=\mathrm{v}(\mathrm{e})
$$

Provamos assim,a validade do algoritmo de incrementos relaxado.No caso em que é imposta a restrição de subaditividade das funçōes consideradas, ou seja,conforme proposto por Johnson, a finitude do algoritmo é também assegurada devido ao seguinte lema.

\section{LEMA 7.1.15}

Seja $k \in \mathbb{N}$ e $\pi(.) \in \Omega^{k} \pi($.$) subaditiva em N$ Então $\pi(.) \in W_{G}$, isto é, $\forall x \in \mathbb{Z}^{q} x \geqq 0 \pi(F x) \leqq\langle d, x\rangle$

\section{Demonstração}

É claro que $\forall j \in\{1,2, \ldots q\} F^{j} \in O_{L}^{k} \cup C_{L}^{k}$.

Como $\pi(.) \in \Omega^{k}, \pi\left(F^{j}\right) \leq g^{k}\left(F^{j}\right) \leq d_{j}$. Mas da subaditividade de $\pi($.$) em N$ vem que $\forall x \in \mathbb{Z}^{q} x \geqq 0 \pi(F x) \leqq \sum_{j=1}^{q} x_{j} \pi\left(F^{j}\right) \leqq<d, x>\mathrm{e}$ segue a afirmação.

Observe ainda que devido à subaditividade da função $v($.$) ,a vali-$ dade do algoritmo ao utilizar funções subaditivas é sempre assegurada.

Tudo que fizemos até então poderia ter sido verificado se a restrição $\pi(e)+\pi\left(f^{0}-e\right)=\pi\left(f^{0}\right) \forall e \in C_{L}^{k}$ também fizesse parte da definição de $\Omega^{k} .0$ único resultado que ficaria inválido ao considerá-la seria este último,que assegura que $\forall k \in \mathbb{N} v(.) \in \Omega^{\mathbf{k}}$, conforme mostra o seguinte exemplo : 
Considere o problema

$\min 10 x_{1}+15 x_{2}$

sa $0.1 x_{1}+0.2 x_{2} \equiv 0.2$

$x_{1} \geqq 0 x_{2} \geqq 0$

Se na primeíra iteração do método $(k=1)$ escolhermos $j_{1}=0.1$ para inclusão em $C^{1}$ teriamos :

$C^{1}=\{0,0.1\} \quad O^{1}=\{0.2\}$

$v(0)=g^{1}(0) v(0.1)=10=g^{1}(0.1) v(0.2)=15=g^{1}(0.2)$

Assim tomando-se $e=0.1 f^{0}=0.2$,

$\pi\left(f^{0}\right) \neq(e)+\pi\left(f^{0}-e\right)$

Como queremos determinar o valor de $v\left(f^{0}\right)$,é natural que a função valor ótimo também possa ser incluida nos conjuntos $\Omega^{k}$,sendo esta a principal razão pela qual a restrição $\pi\left(f^{0}\right)=\pi\left(f^{0}-e\right)+\pi(e) e \in C_{L}^{k}$ foi excluida da definição de $\Omega^{k}$.Além disso, em termos operacionais a inclusão desta condição na definição de $\Omega^{k}$ deve acarretar algum trabalho adicional no processo de determinação da sequência $\pi^{k}(.)_{k \in \mathbb{N}}$ *

\subsection{CONCLUSÃO}

Neste capítulo definimos um algoritmo bastante semelhante a $A^{*}$ para a resoluçäo do problema de grupos.Apesar de termos verificdo que sob hipóteses não muito restritivas a aplicação do algoritmo é sempre possível,

não determinamos ainda um método simples,em termos operacionais,de gerar uma sequência de funções $\left\{\pi^{k}(.)\right\}_{k \in \mathbb{N}}$ com as propriedades exigidas pelo algoritmo.

Nosso trabalho prossegue,procurando determinar uma metodologia para a construção de tal sequência. Verificamos, não obstante, que de acordo com a sequência de funções utilizada,relações muito fortes entre $A^{*}$ e 0 método de incrementos é mantida.

* Parece-nos que a restrição tenha sido adotada nos trabalhos de Johnson por estar relacionada com a caracterização de faces do poliedro que descreve o conjunto de pontos viáveis do problema (Vide [Jo80A],Capítulo IV , §3) 
Capítulo 8

\section{Uma relacão entre $A^{*} \mathbf{e} 0$ algoritmo de incrementos}

Além de serem métodos que rotulam iterativamente elementos de um dado conjunto,tanto $A^{*}$ quanto o algoritmo de incrementos, estão fortemente relacionados com dualidade, dentro de um conceito amplo de problema dual.

A questão sobre qual é o melhor método a ser utilizado na resolução de um problema de grupos só pode ser respondida se dispusermos de um processo bem deteminado de construção de heurísticas e funçōes viáveis em problemas duais.Esta resposta esta associada ao conhecimento do "melhor" processo para tal construção, o que não se sabe a priori.

Este capítulo é orientado no sentido de comparar os dois métodos, sem procurar determinar qual é o melhor,porém verificando condições sob as quais ambos se comportam como um só algoritmo. 


\subsection{UMA CONSTRUÇÃO DE FUNÇÕES SUBADITIVAS}

Vamos assumir a hipótese 7.1.1 do capítulo anterior,a fim de que possamos assegurar a finitude dos algoritmos $A^{*}$ e de incrementos relaxado na resolução do problema de grupos (PG).

O estudo de funções subaditivas algumas vezes está relacionada,à determinição de faces de poliedros,através da geração de equações válidas, isto é, que não excluam elementos do conjunto de pontos viáveis considerado (correspondendo a noção de cortes em programação inteira) [Jo79] - Trabalhos interessantes de Burdet e Johnson ([BJ74], [BJ77]) geram funçōes subaditivas a partir da construção de funçốes chamadas gauges,as quais podem ser obtidas facilmente.

Estudaremos uma construção de funçōes subaditivas aplcável ao método de incrementos, e bastante simples em termos operacionais. 0 processo construtivo proposto baseia-se em [BJ74].

\section{DEFINIÇÄO 8.1.1}

Dado $\Gamma \subset \mathbb{R}^{q}$ e finito, e duas funções

$\hat{g}: \mathbb{R}^{\mathfrak{q}} \rightarrow \mathbb{R}$ e $\Delta: \mathbb{R}^{q} \rightarrow \mathbb{R} \cup\{-\infty\}$,definimos $\pi_{\Gamma}($.$) por:$

$$
\begin{gathered}
\pi_{\Gamma}: \mathbb{R}^{q} \rightarrow \mathbb{R} \cup\{-\infty\} \\
\pi_{\Gamma}(y)=\inf _{e \in \Gamma}\{\hat{g}(e)+\Delta(y-e)\}
\end{gathered}
$$

Para esta função vale :

\section{PROPOSIÇÃO 8.1.2}

Seja $\pi_{\Gamma}($.$) a função definida em 8.1.1.$

Se $\Delta($.$) é subaditiva em \mathbb{R}^{\mathrm{p}}$ e $\pi_{\Gamma}(e+f) \leqq \hat{g}(e)+\hat{g}(f) \forall(e, f) \in \Gamma \times \Gamma$ então $\pi$ (.) é subaditiva. 
Demonstração

Seja $\left(y_{1}, y_{2}\right) \in \mathbb{R}^{q} \mathbb{x}^{q}$

Se $\pi_{\Gamma}\left(y_{1}\right)=-\infty$ então $\exists \hat{e} \in \Gamma \mid \hat{g}(\hat{e})+\Delta\left(y_{1}-\hat{e}\right)=-\infty$ Assim, $\pi_{\Gamma}\left(y_{1}+y_{2}\right)=$

$$
\begin{aligned}
& =\inf _{e \in \Gamma}\left\{\hat{g}(e)+\Delta\left(y_{1}+y_{2}-e\right)\right\} \\
& \leqq \inf _{e \in \Gamma}\left\{\hat{g}(e)+\Delta\left(y_{1}+e\right)+\Delta\left(y_{2}\right)\right\} \\
& =-\infty
\end{aligned}
$$

e portanto

$$
\pi_{\Gamma}\left(y_{1}+y_{2}\right) \leqq \pi_{\Gamma}\left(y_{1}\right)+\pi_{\Gamma}\left(y_{2}\right)
$$

Da mesma forma se $\pi_{\Gamma}\left(y_{2}\right)=-\infty$ segue que

$$
\pi_{\Gamma}\left(y_{1}+y_{2}\right) \leqq \pi_{\Gamma}\left(y_{1}\right)+\pi_{\Gamma}\left(y_{2}\right)
$$

Se $\pi_{\Gamma}\left(y_{1}\right) \in \mathbb{R}$ e $\pi_{\Gamma}\left(y_{2}\right) \in \mathbb{R}$, considere $\left(e_{1}, e_{2}\right) \in \Gamma \times \Gamma \mid$

$$
\begin{aligned}
& \pi_{\Gamma}\left(y_{1}\right)=\hat{g}\left(e_{1}\right)+\Delta\left(y_{1}-e_{1}\right) \\
& \pi_{\Gamma}\left(y_{2}\right)=\hat{g}\left(e_{2}\right)+\Delta\left(y_{2}-e_{2}\right)
\end{aligned}
$$

Então

$$
\begin{gathered}
\pi_{\Gamma}\left(y_{1}\right)+\pi_{\Gamma}\left(y_{2}\right)= \\
\hat{g}\left(e_{1}\right)+\hat{g}\left(e_{2}\right)+\Delta\left(y_{1}-e_{1}\right)+\Delta\left(y_{2}-e_{2}\right)
\end{gathered}
$$

e da subaditividade de $\Delta($.$) vem que$

$$
\begin{gathered}
\pi_{\Gamma}\left(y_{1}\right)+\pi_{\Gamma}\left(y_{2}\right) \\
\geqq \hat{g}\left(e_{1}\right)+\hat{g}\left(e_{2}\right)+\Delta\left(y_{1}+y_{2}-e_{1}-e_{2}\right)=
\end{gathered}
$$


Capítulo 8: Uma relacão entre $A^{*}$ e o algoritmo de incrementos

$$
\begin{gathered}
=\hat{g}\left(e_{1}\right)+\hat{g}\left(e_{2}\right)+\Delta\left(y_{1}+y_{2}-\left(e_{1}+e_{2}\right)\right) \geqq \\
\geqq \pi_{\Gamma}\left(e_{1}+e_{2}\right)+\Delta\left(y_{1}+y_{2}-\left(e_{1}+e_{2}\right)\right)
\end{gathered}
$$

isto é,

$$
\begin{gathered}
\pi_{\Gamma}\left(e_{1}+e_{2}\right)+\Delta\left(y_{1}+y_{2}-\left(e_{1}+e_{2}\right)\right) \leqq \\
\pi_{\Gamma}\left(y_{1}\right)+\pi_{\Gamma}\left(y_{2}\right)
\end{gathered}
$$

(I) $\{1,2\}$ temos

Por outro lado,

$\forall\left(z_{1}, z_{2}\right) \in \mathbb{R}^{q} \times \mathbb{R}^{q} \operatorname{com} \pi\left(z_{j}\right)=\hat{g}\left(w_{j}\right)+\Delta\left(z_{j}-w_{j}\right) w_{j} \in \Gamma j \in$

$$
\begin{gathered}
\pi_{\Gamma}\left(z_{1}\right)=\inf _{e \in \Gamma}\left\{\hat{g}(e)+\Delta\left(z_{1}-e\right)\right\} \leqq \\
\leqq \hat{g}\left(w_{2}\right)+\Delta\left(z_{1}-w_{2}\right)= \\
=\hat{g}\left(w_{2}\right)+\Delta\left(z_{1}-z_{2}+z_{2}-w_{2}\right) \leqq \\
\leqq \hat{g}\left(w_{2}\right)+\Delta\left(z_{1}-z_{2}\right)+\Delta\left(z_{2}-w_{2}\right)= \\
\pi_{\Gamma}\left(z_{2}\right)+\Delta\left(z_{1}-z_{2}\right)
\end{gathered}
$$

Aplicando essa desigualdade para $z_{1}=y_{1}+y_{2} \quad z_{2}=e_{1}+e_{2}$ vem

$$
\pi_{\Gamma}\left(y_{1}+y_{2}\right) \leqq \pi_{\Gamma}\left(e_{1}+e_{2}\right)+\Delta\left(y_{1}+y_{2}-\left(z_{1}+z_{2}\right)\right)
$$

e de (I) segue que

$$
\pi_{\Gamma}\left(y_{1}+y_{2}\right) \leqq \pi_{\Gamma}\left(y_{1}\right)+\pi_{\Gamma}\left(y_{2}\right)
$$


A vantagem ao utilizarmos tal função reside no fato que conhecendo -se funções triviais ,como por exemplo as lineares,podemos construir funções subaditivas que garantem a convergência do método de incremento subaditivo. Na verdade vamos trabalhar com funçōes que nos "octantes" de $\mathbb{R}^{p}$ se comportem como as funçōes lineares.

\section{DEFINIÇÃO 8.1.3}

Dado $p \in \mathbb{N}$ e $j \in \mathbb{N} 0 \leqq j \leqq 2^{p}-1$ denotamos por $Q_{j}$ ao j-ésimo octante de $\mathbb{R}^{p}$,isto é

j na base 2$\}$.

$Q_{j}=\left\{u \in \mathbb{R}^{\mathrm{p}} \mid \mathrm{u}_{\mathrm{i}} \geqq 0 \Longleftrightarrow \mathrm{j}_{2}^{\mathrm{i}}=1\right.$ onde $j_{2}$ é a representação de

Nestas condições temos

\section{PROPOSIÇÃO 8.1.4}

Nas condições da proposição anterior

$$
\mathbb{R}^{p}=U_{j=0}^{2^{p}-1} Q_{j}
$$

Demonstração

i) $\cup_{j=0}^{2^{p}-1} Q_{j} \subset \mathbb{R}^{p}$

Imediato

ii) $\mathbb{R}^{p}=\cup_{j=0}^{2^{p}-1} Q_{j}$

Seja $x \in \mathbb{R}^{p}$ e considere $t \in\{0,1\}^{p}, t_{i}= \begin{cases}1 & \text { se } x_{i}<0 \\ 0 & \text { c.c }\end{cases}$

Sendo $j=\sum_{k=1}^{p} t_{i} 2^{(i-1)} \leqq 2^{p}-1$ é imediato, por construção, que $x \in Q_{j}$ e segue a afirmação 
Capítulo 8: Uma relacão entre $A^{*}$ e o algoritmo de incrementos 6 6

Para construirmos funções que sejam lineares nestes octantes, utilizaremos a seguinte definição

\section{DEFINIÇÃO 8.1.5}

Denotamos por $\mathbf{1}^{j}, 0 \leqq j \leqq 2^{p}-1$, ao vetor de $\mathbb{R}^{p}$ tal que

$$
\begin{gathered}
\mathbb{1}^{\mathrm{j}} \in \mathbf{Q}_{\mathrm{j}} \\
|\mathbf{H}|=1 \quad \forall i \in\{1,2, \ldots \mathrm{p}\}
\end{gathered}
$$

Nestas condições temos

LEMA 8.1.6

Seja $\Delta_{1}($.$) definida por :$

$$
\begin{gathered}
\Delta_{1}: \mathbb{R}^{p} \rightarrow \mathbb{R} \\
\Delta_{1}(u)=\max _{0 \leqq j \leqq 2^{p}-1}\left\{<\mathbb{1}^{j}, u>\right\}
\end{gathered}
$$

Então $\Delta_{1}()=.\|\cdot\|_{1} *$

Demonstração

Seja $u \in \mathbb{R}^{p}$.

Como $\mathbb{R}^{\mathrm{P}}=\mathrm{U}_{\mathbf{j}=0}^{2^{\mathrm{P}}-1} \mathrm{Q}^{\mathbf{j}}, \exists \vec{j}, 0 \leqq \hat{j} \leqq 2^{p}-1$ tal que $u \in Q_{\bar{j}}$. Assim,

* Por definiçã̃o

$\| \begin{array}{ll}\|_{1}: \mathbb{R}^{p} \rightarrow \mathbb{R}_{+} \\ x \|_{1}=\sum_{j=1}^{q}\left|x_{i}\right|\end{array}$ 


$$
\begin{gathered}
\left\langle\mathbf{1}^{\top}, \mathrm{u}\right\rangle= \\
=\sum_{i=1}^{p} \mathbb{1}_{\mathrm{i}}^{\bar{j}} \mathrm{u}_{\mathrm{i}}= \\
=\sum_{i=1}^{p}\left|\mathbf{1}_{\mathrm{i}}^{\bar{j}} \mathrm{u}_{\mathrm{i}}\right|= \\
=\sum_{i=1}^{p}\left|u_{i}\right|=\|u\|_{1}
\end{gathered}
$$

Por outro lado, $\forall j 0 \leqq j \leqq 2^{p}-1$ temos

$$
\begin{gathered}
<\mathbf{1}^{\mathrm{j}}, \mathrm{u}>=\sum_{\mathrm{j}=1}^{\mathrm{p}} \mathbf{1}_{\mathrm{i}}^{\mathrm{j}} \mathrm{u}_{\mathrm{i}} \leqq \\
\leqq \sum_{i=1}^{p}\left|\mathbf{1}_{\mathrm{i}}^{\mathrm{j}} \mathrm{u}_{\mathrm{i}}\right|= \\
=\sum_{i=1}^{p}\left|u_{i}\right|=\|u\|_{1}
\end{gathered}
$$

$\log 0, \Delta_{1}(u)=\max _{0 \leqq j \leqq 2^{p}-1}\left\{\left\langle\mathbb{1}^{\mathrm{j}}, \mathrm{u}\right\rangle\right\}==\left\{\left\langle\mathbf{1}^{\mathrm{j}}, \mathrm{u}\right\rangle\right\}=\|\mathrm{u}\|_{1}$

Assim,podemos escrever a norma, $\left\|_{0}\right\|_{1}$, como o máximo de um número finito de funções lineares. $O$ trabalho de Burdet e Johnson [BJ74] utiliza implicitamente no método de icrementos subaditivos, funções semelhante a estas, na forma $\Delta(u)=\max _{0 \leqq j \leqq 2^{p}-1}\left\{\left\langle l^{j},\right\rangle\right\} \operatorname{com} l^{j} \in \mathbb{R}^{\mathrm{p}}$, $, \forall j, 0 \leqq j \leqq 2^{p}-1$

Ao invés de considerar $F^{j} \in[0,1[p$,esses autores trabalham com hipercubo unitário $H u=\left\{u \in \mathbb{R}^{\mathrm{p}} \mid \mathrm{f}_{\mathrm{i}}^{0}-1 \leqq u_{\mathrm{i}} \leqq \mathrm{f}_{\mathrm{i}}^{0} \mathrm{i} \in(1, \ldots, \mathrm{p})\right\}\left(f^{0} \geqq 0\right.$ por hipótese).

Dado então $\alpha \in \mathbb{R}^{p}, \alpha \geqq 0$, considera-se 


$$
\sum_{i=1}^{p} \alpha_{i} f_{i}^{0}\left(1-f_{i}^{0}\right)=\alpha_{0}
$$

e define-se $D($.$) por$

$$
D(u)=\sum_{i=1}^{p} \alpha_{i} \sigma_{i}\left(u_{i}\right) u_{i}
$$

onde

$$
\sigma\left(u_{i}\right)=\left\{\begin{array}{cl}
1-f_{i}^{0} & \text { se } u_{i} \geqq 0 \\
-f_{i}^{0} & \text { cc }
\end{array}\right.
$$

Pode-se verificar que esta função é linear nos octantes de $\mathbb{R}^{p}$, $D(u)=\left\langle l^{j}, u\right\rangle$ em $Q_{j}$ onde

$$
l_{i}^{j}= \begin{cases}\alpha_{i}\left(1-f_{i}^{0}\right) & \text { se } \alpha_{i} \geqq 0 \\ -\alpha_{i} f_{i}^{0} & \text { cc }\end{cases}
$$

A idéia dos autores é construir funçóes $\pi($.) subaditivas a partir de $\mathrm{D}\left(\right.$.).Através da geraçào de uma sequência de vetores de $\mathbb{R}^{\mathrm{p}},\left\{\alpha^{k}\right\}_{k \in \mathbb{N}}$, obtem -se a sequência de funções $\left\{\pi^{k}(.)\right\}_{k \in \mathbb{N}}$, que satisfaz as condiçốes impostas pelo algoritmo de incrementos subaditivos.

No trabalho citado,não fica claro o processo de construção de $\left\{\alpha^{k}\right\}_{k \in \mathbb{N}}$ utilizado.Iremos aqui,elaborar uma construção bastante simples de tais elementos,sem considerar o hipercubo $\mathrm{H}_{u}$.

\section{PROPOSIÇÃO.8.1.7}

Considere a sequência de vetores de $\mathbb{R}^{\mathrm{p}},\left\{l^{j}\right\}_{j=0}^{2^{p}-1}$ definida por $l^{j}=\alpha \mathbf{1}^{\mathbf{j}}, \quad$ para algum $\alpha \in \mathbb{R}_{+}$

Se $\Delta: \mathbb{R}^{p} \rightarrow \mathbb{R}$

$$
\Delta(u)=\max _{0 \leqq j \leqq 2^{p}-1}\left\{\left\langle l^{j}, u\right\rangle\right\}
$$

então
i) $\Delta()=.\alpha\|\cdot\|_{1}$
ii) $\Delta($.$) é linear em Q_{j} \forall j 0 \leqq j \leqq 2^{p}-1$
iii) $\Delta($.$) é convexa$ 
iv) $\Delta($.$) é subaditiva$

Demonstração

i) $\Delta()=.\alpha\|\cdot\|_{1}$

$\forall u \in \mathbb{R}^{\mathrm{p}}, \Delta(\mathrm{u})=: \max _{0 \leqq \mathrm{j} \leqq 2(\mathrm{p})-1}\left\{\left\langle l^{j}, u\right\rangle\right\}=$

$\max _{0 \leqq j \leqq 2(p)-1}\left\{\left\langle\alpha \mathbf{l l}^{\mathbf{j}}, \mathrm{u}\right\rangle\right\}=$

$\max _{0 \leqq j \leqq 2(p)-1}\left\{<1^{j}, u>\right\}=$

$=\alpha \Delta_{1}(u)=$

$=\alpha\|u\|_{1}$

ii) $\Delta($.$) linear em Q_{j}$

Seja $u \in Q_{\vec{j}}$.

Como $\Delta(u)=\alpha \Delta_{1}(u)=\alpha\left\langle\mathbf{1}^{\mathrm{j}}, \mathrm{u}\right\rangle$ conforme estudamos na demonstraçäo do lema 7.2.7 temos entäo

$\Delta(u)=\left\langle\alpha \mathbb{1}^{j}, u>\right.$ sendo portanto linear em $Q_{\bar{j}}$

iii) $\Delta($.$) convexa$

Trivial,pois $\Delta()=.\alpha\|\cdot\|_{1}$ com $\alpha \geqq 0$

iv) $\Delta($.$) é subaditiva$

Sejam $\left(u_{1}, u_{2}\right) \in \mathbb{R}^{\mathrm{P} x} \mathbb{R}^{\mathrm{P}}$ Então

$\Delta\left(u_{1}, u_{2}\right)=\alpha\left\|u_{1}+u_{2}\right\|_{1} \leqq$

$\alpha\left\|u_{1}\right\|_{1}+\alpha\left\|u_{2}\right\|_{1}=$

$=\Delta\left(u_{1}\right)+\Delta\left(u_{2}\right)$

Rockafellar [Ro70] denomina "gauge",qualquer função não negativa,convexa e positivamente homogenea (isto é,tal que $\forall \lambda \in \mathbb{R}^{+} f(\lambda x)=$ $\lambda f(x))$.Convexidade e homogeneidade são condiçóes suficientes para assegurar a subaditividade,sendo esta uma razão pela qual alguns trabalhos procuram estudar gauges. 0 processo construtivo que discrevemos utiliza particuralizações do conceito de gauge. 


\section{LEMA 8.1.8}

Sejam $\Delta_{a}(). e \Delta_{b}$ construidas segundo a proposição 8.1.7, $\Delta_{a}()=.\alpha_{a} \Delta_{1}(.) \quad \Delta_{b}=\alpha \Delta_{1}(.) \quad \alpha_{a} \leqq \alpha_{b}$ Sendo $\pi_{a}($.$) e \pi_{b}($.$) definidas por$ $\pi_{a}: \mathbb{R}^{\mathrm{p}} \rightarrow \mathbb{R}$

$$
\pi_{a}(u)=\min _{e \in C}\left\{\hat{g}(e)+\Delta_{a}(u-e)\right\}
$$

*

$$
\begin{gathered}
\pi_{b}: \mathbb{R}^{p} \rightarrow \mathbb{R} \\
\pi_{b}(u)=\min _{e \in C}\left\{\hat{g}(e)+\Delta_{b}(u-e)\right\}
\end{gathered}
$$

com $C \subset \mathbb{R}^{\mathrm{p}} \mathbf{C} \neq \emptyset$, e $\hat{g}: \mathbb{R}^{\mathrm{p}} \rightarrow \overline{\mathbb{R}}$ tem-se $\pi_{a}(.) \leqq \pi_{b}($.

\section{Demonstração}

Trivial

Verificamos assim que que se tomarmos uma sequência crescente de elementos $\left\{\alpha^{k}\right\}_{k \in \mathbb{N}}$, a correspondente sequência $\left\{\pi^{k}().\right\}$, é não decrescente (desde que não alteremos o conjunto sobre o qual é feita a minimização).Para que essas idéias se apliquem ao método de incrementos é necessário que o conjunto $C$ possa ser alterado com as iterações.

\section{PROPOSIÇÃO 8.1 .9}

Sejam $\pi^{1}($.$) e \pi^{2}($.$) definidas por$

$$
\pi^{j}(u)=\min _{e \in C^{j}}\{\hat{g}(e)+\Delta(u-e)\} j \in\{1,2\}
$$

onde $\Delta($.$) é construida conforme 8.1.7, C^{1} \subset \mathbb{R}^{p} C^{2} \subset \mathbb{R}^{\mathrm{p}}$ são conjuntos finitos e não vazios $C^{2}=C^{1} \cup\{\hat{e}\}$, e $\hat{g}($.$) uma função dada. Se$ $\pi^{1}(\hat{e})=\hat{g}(\hat{e})$ então $\pi^{1}()=.\pi^{2}($.$) .$

* Note que neste caso podemos trabalhar com mínimo ao invés de infimo pois as funçòes $\Delta($.$) consideradas só assumem valores reais e C$ é finito 
Demonstração

Seja $u \in \mathbb{R}^{p}$

i.) Se $\exists e_{2} \in C^{1} \mid \pi^{2}(u)=\hat{g}\left(e_{2}\right)+\Delta\left(u-e_{2}\right)$

para algum $e_{2} \in C^{1}$ então claramente,

$$
\begin{gathered}
\pi^{2}(u)=\min _{e \in C^{2}}\{\hat{g}(e)+\Delta(u-e)\}= \\
=\min _{e \in C^{1}}\{\hat{g}(e)+\Delta(u-e)\}= \\
\pi^{\mathbb{1}}(u)
\end{gathered}
$$

ii.) Se $\pi^{2}(u)=\hat{g}(\hat{e})+\Delta(u-\hat{e}), \hat{e} \in C^{2} \backslash C^{1}$, tomemos

$e_{1} \in C^{1} \mid \pi(\hat{e})=\hat{g}\left(e_{1}\right)+\Delta\left(\hat{e}-e_{1}\right)$

$$
\begin{gathered}
\pi^{2}(u) \leqq \hat{g}\left(e_{1}\right)+\Delta\left(u-e_{1}\right)= \\
=\hat{g}\left(e_{1}\right)+\Delta\left(u-\hat{e}+\hat{e}-e_{1}\right)= \\
\leqq \hat{g}\left(e_{1}\right)+\Delta(u-\hat{e})+\Delta\left(\hat{e}-e_{1}\right)= \\
=\pi^{1}(\hat{e})+\Delta(u-\hat{e})
\end{gathered}
$$

Mas por hipótese, $\pi^{1}(\hat{e})=g(\hat{e})$ e, portanto, $\pi^{2}(u) \leqq \hat{g}(\hat{e})+\Delta(u-\hat{e})=\pi^{2}(u)$

Assim vale a igualdade nas expressóes acima, $\mathrm{e}$

$\pi^{2}(u)=\hat{g}\left(e_{1}\right)+\Delta\left(u-e_{1}\right) \geqq \pi^{1}(u)$

Ora, mas $\pi^{2}(u) \leqq \pi^{1}(u)$ por construção, uma vez que

$C^{1} \subset C^{2}$. Logo $\pi^{1}(u)=\pi^{2}(u)$.

$\mathrm{De}$ (i) e (ii) segue a tese.

A aplicação deste resultado ao algoritmos de incrementos é imediata. Suponhamos que para alguma iteração do método, tenhamos

$$
\pi^{k}(u)=\min _{e \in C^{k}}\left\{g^{k}(e)+\Delta^{k}(u-e)\right\}
$$




$$
\Delta^{k}(.)=\alpha^{k}\|\cdot\|_{1}, \pi^{k}(.) \in \Omega^{k-1}
$$

Se pudermos obter $\Delta^{k+1}()=.\alpha^{k+1}\left\|_{.}\right\|_{1}$, tal que

$\hat{\pi}(u)=\min _{e \in C^{k}}\left\{g^{k}(e)+\Delta^{k+1}(u-e)\right\}$ satisfaça $\hat{\pi}(\hat{e})=g^{k}(\hat{e})$, para algum $\hat{e} \in O^{k}$, então $\pi^{k+1}(u)=\min _{e \in C^{k+1}}\left\{g^{k}(e)+\Delta^{k+1}(u-e)\right\}$ satisfaz $\hat{\pi}()=.\pi^{k+1}($.$) , onde C^{k+1}=C^{k} \cup\{\hat{e}\}$. Assim, teremosdeterminadoumanova funcão. com características semelhantes às de $\pi^{k}($.$) .$

Exemplificando,

EXEMPLO 8.1.10

Considere o seguinte problema:

$\min 15 x_{1}+20 x_{2}$

sa $0.25 x_{1}+0.5 x_{2} \equiv 0.75$

$x_{1} \geqq 0, x_{2} \geqq 0$ inteiros

$k=0$

Seja $\alpha^{0}=0, l_{j}^{0}=0 j \in\{0,1\}$.Sendo

$\Delta^{0}(u)=\max _{j \in\{0,1\}}\left\{\left\langle l_{j}^{0}, u\right\rangle\right\}$.Temos

$\Delta^{0}(.) \equiv 0$ e $\pi^{0} \equiv 0$ pois $C^{0} \equiv\{0\}$

$k=1$

Tomemos $\alpha^{1}=40$ e portanto $l_{1}^{1}=-40$ e $l_{2}^{1}=40$.

Tomemos então $\Delta^{1}(u)=40\|u\|_{1}$ e

$\pi^{1}(u)=\Delta^{1}(u)=40\|u\|_{1}$.Com isso

$\pi^{1}(0)=0 \pi^{1}(0.25)=10 \pi^{1}(0.5)=20$

Escolhemos 0.25 para fechamento e temos $C^{1}=\{0,0.5\}$

$O^{1}=\{0.75,0.25\}$

$k=2$

$\pi^{2}(u)=\min _{e \in\{0,0.5\}}\left\{g^{2}(e)+\Delta^{2}(u-e)\right\}$

$\Delta^{2}(u)=\max \left\{\left\langle l_{1}^{2}, u\right\rangle,\left\langle l_{2}^{2}, u\right\rangle\right\}$

Tomando-se alpha ${ }^{2}=15 / 25$ temos $l_{1}^{2}=-15 / 25 l_{1}^{2}=15 / 25 \mathrm{e}$ $\Delta^{2}(u)=15 / 25\|u\|_{1}$, donde 
$\pi^{2}(0)=0 \pi^{2}(0.5)=20 \pi^{2}(0.25)=15 \pi^{2}(0.75)=35$

Posso fechar 0.25 e então:

$$
\begin{aligned}
& C^{2}=\{0,0.5,0.25\} \\
& O^{2}=\{0.75\}
\end{aligned}
$$

$k=3$

$$
\pi^{3}(.)=\pi^{2}(.)
$$

Fechando 0.75 tem-se $O^{3}=\emptyset$ e o algoritmo termina com $v(0.75)=35$

Nosso problema então é a determinação da sequência não decrescente de números reais $\left\{\alpha^{k}\right\}_{k \in \mathbb{N}}$ que assegurem que se $\pi^{k}(.) \in \Omega^{k}$ então $\pi^{k+1}(.) \in \Omega^{k+1}$

\section{DEFINIÇÄO 8.1.11}

Seja $k \in \mathbb{N}$ numa iteração qualquer do método de incrementos $\mathbf{e}$ $\pi^{k}($.$) definida por :$

$\pi(u)=\min _{e \in C^{k}}\left\{g^{k}(e)+\Delta^{k}(u-e)\right\}$

onde $\Delta^{k}()=.\alpha^{k}\|\cdot\|_{1}$ para algum $\alpha^{k} \in \mathbb{R}$.

Chamamos de $\mu^{k}$ ao elemento de $\overline{\mathbb{R}}$

$\mu^{k}=\inf \left\{\frac{g^{k}(e)-\left|g^{k}\left(e_{1}\right)+\Delta\left(e-e_{1}\right)\right|}{\left\|e-e_{1}\right\|} \mid e \in O^{k} e_{1} \in V^{k}(e)\right\}$
onde $V^{k}(e)=\left\{e_{1} \in C^{k} \mid g^{k}\left(e_{1}\right)+\Delta^{k}\left(e-e_{1}\right)<g^{k}(e)\right\}$ Nessas condiçóes denominamos $\epsilon^{k}$ ao elemento

$$
\epsilon^{k}=\left\{\begin{array}{cl}
\mu^{k} & \text { se } \mu^{k} \in \mathbb{R} \\
0 & \text { c.c. }
\end{array}\right.
$$

Com esta definição é possível construir a partir de $\pi^{k}(.) \in \Omega^{k-1}$, uma nova funçäo $\pi^{k+1}($.$) que satisafaça às propriedades necessárias à convergêncią$ do método. 
Seja $\pi^{k}($.$) e \epsilon^{k}$ conforme definição anterior e

$$
\begin{aligned}
& \pi: \mathbb{R p} \rightarrow \mathbb{R} \\
& \quad \pi(u)=\min _{e \in C^{k}}\left\{g^{k}(e)+\Delta(u-e)\right\} \text { onde } \\
& \Delta(.)=\alpha\|\cdot\|_{1} \alpha=\alpha^{k}+\epsilon^{k}
\end{aligned}
$$

Então

$$
\pi(\cdot) \geqq \pi^{k}(\cdot)
$$

Demonstração afirmação.

Por construção $\epsilon^{k} \geqq 0$.Como $\alpha=\alpha^{k}+\epsilon^{k}$ vem que $\alpha \geqq \alpha^{k}$ e segue a

Além disso temos

\section{LEMA.8.1.13}

Nas condições do lema anterior,sendo $\pi^{k}(.) \in \Omega^{k-1}$

Então

$$
\forall e \in C^{k} \pi(e)=g^{k}(e)
$$

\section{Demonstração}

Como $\pi^{k}(.) \in \Omega^{k-1}$,temos que $\forall l \in C^{k} \pi^{k}(e)=g^{k}(e)$ e do resultado anterior $\pi(e) \geqq \pi^{k}(e)=g^{k}(e)$

Mas $\pi(e)=\min _{\hat{\varepsilon} \in C^{k}}\left\{g^{k}(\hat{e})+\Delta(e-\hat{e})\right\} \leqq$

$\leqq g(e)+\Delta(e-e)=g^{k}(e)$.

Assim, $g^{k}(e) \leqq \pi(e) \leqq g^{k}(e)$ esegueaafirmacão

Não somente no conjunto de elementos fechados a função $\pi($.$) é$ bem comportada. 


\section{LEMA.8.1.14.}

Nas condições do LEMA.8.1.12 sendo $\pi^{k}(.) \in \Omega^{k-1}$ temos

$$
\forall e \in O^{k} \pi(e) \leqq g^{k}(e)
$$

\section{Demonstração}

Suponhamos que $\exists \bar{e} \in O^{k} \mid \pi(\bar{e})>g^{k}(\bar{e})$. Sendo $w \in C^{k}$ tal que $\pi^{k}(\bar{e})=g^{k}(w)+\Delta^{k}(\bar{e}-w)$ temos $g^{k}(\bar{e})=\pi(\bar{e}) \leqq g^{k}(w)+\Delta(\bar{e}-w)=$ $g^{k}(w)+\Delta^{k}(\bar{e}-w)+\epsilon^{k}\|\bar{e}-w\|$

Assim,

$\epsilon^{k}=\frac{\underline{g}^{k}(\bar{e})-\| g^{k}(w)+\Delta^{k}(\bar{e}-w) \mid}{\|\bar{\varepsilon}-w\|_{1}}$

contrariando a construção de $\epsilon^{k}$

Estamos procurando verificar que a função $\pi(.) \in \Omega^{k}$ e portanto devemos ter $\pi($.$) subaditiva.$

\section{LEMA.8.1.15}

Nas condições do lema 8.1.12, se $\pi^{k}(.) \in \Omega^{k-1}$ então $\pi($.$) é subaditva.$

\section{Demonstração}

Sendo $\Delta()=.\alpha\|\|_{1}$ subaditiva, basta verificarmos subaditividade de $\pi($.$) em C^{k}$. Sejam então $\left(u_{1}, u_{2}\right) \in C^{k} x C^{k}$.

Claramente $u_{1}+w_{2} \in O^{k} \cup C^{k}$ por construção. Temos então: $\pi(u)=\pi\left(u_{1}+u_{2}\right) \leqq g^{k}\left(u_{1}+u_{2}\right)$ pelos lemas 8.1.13 e 8.1.14.

Mas do processo construtivo do algoritmo

$$
g^{k}\left(u_{1}+u_{2}\right) \leqq g^{k}\left(u_{1}\right)+g^{k}\left(u_{2}\right)
$$

e segue que

$$
\pi\left(u_{1}+u_{2}\right) \leqq \pi\left(u_{1}\right)+\pi\left(u_{2}\right)
$$


Para que possamos incluir $\pi($.$) em \Omega^{k}$ é necessário que se imponha ainda que $\pi(e) \leqq g^{k+1}(e) \forall e \in O^{k}$, uma vez que os valores de $g^{k}($.$) podem ser$ alterados com as iteraçôes.

\section{LEMA.8.1.16}

Nas condiçôes do lema 8.1.12 tem-se que

se $\pi^{k}(.) \in \Omega^{k-1}$ então $\forall e \in O^{k+1} \pi(e) \leqq g^{k+1}(e)$

\section{Demonstração}

Seja $e \in O^{k+1}$ Se $e \in O^{k} \quad$ e $g^{k}(e)=g^{k+1}(e)$ nada a fazer,a afirmação segue de 8.1.14.

Caso contrário para algum par $\left(e_{1}, e_{2}\right) \in O^{k} x O^{k}$ tem-se que $e=$ $e_{1}+e_{2}, g^{k+1}(e)=g^{k}\left(e_{1}\right)+g^{k}(e)$ Da subaditividade de $\pi($.$) temos então que$ $\pi(e)=\pi\left(e_{1}+e_{2}\right) \leqq \pi\left(e_{1}\right)+\pi\left(e_{2}\right)=g^{k}\left(e_{1}\right)+g^{k}\left(e_{2}\right)=g^{k+1}(e)$ e segue a afirmação.

A única restrição que devemos ainda impor sobre a função $\pi($.$) é$ que $\pi\left(e^{*}\right)=g^{k}\left(e^{*}\right)$ para algum $e^{*} \in O^{k} . \mathrm{Na}$ forma pela qual construimos $\pi($.$) não necessariamente obtemos tal propriedade,porém a repetição deste$ processo construtivo permite que obtenhamos uma função que satisfaça esta condição.

\section{FATO.8.1.17}

$$
\begin{aligned}
& \text { Sendo }\left(e_{1}^{k}, e_{2}^{k}\right) \in O^{k} x\left(C^{k} \backslash O^{k}\right) \text { tal que } \\
& \epsilon=\frac{\left.g^{k}\left(e^{k}\right)-g^{k}\left(e_{1}\right)+\Delta^{k}\left(e^{k}, e_{1}^{k}\right)\right]}{\left\|\left(e^{k}, e_{1}^{k}\right)\right\|_{3}} \\
& \text { tem-se que }
\end{aligned}
$$




$$
g^{k}\left(e^{k}\right)=g^{k}\left(e_{1}^{k}\right)+\Delta\left(e^{k}-e_{1}^{k}\right)
$$

\section{Demonstração}

Como

segue trivialmente

$$
g^{k}\left(e_{1}^{k}\right)+\Delta\left(e^{k}, e_{1}^{k}\right)=g^{k}\left(e_{1}^{k}\right)+\Delta\left(e^{k}, e_{1}^{k}\right)+\Delta\left\|e^{k}-e_{1}^{k}\right\| \text { e a afirmação }
$$

Além disso temos

FATO.8.1.18

Nas condições do lema 8.1.12, dado $\pi^{k}(.) \in \Omega^{k-1}$

Se $\exists e \in O^{k} \mid V(e)=\emptyset$ entäo $\pi^{k}(e)=g^{k}(e)$

Demonstração

Como $\pi^{k}(.) \in \Omega^{k-1}$ temos que $\forall e \in O^{k} \pi^{k}(e) \leq g^{k}(e)$

Logo,se $V(e)=\emptyset$ entäo $\forall e \in C^{k} g^{k}\left(e_{1}\right)+\Delta^{k}\left(e-e_{1}\right) \geq g^{k}(e)$, donde segue que $\pi^{k}(e)=\inf _{e_{1} \in C^{k}}\left\{g^{k}\left(e_{1}\right)+\Delta\left(e-e_{1}\right)\right\} \geqq g^{k}(e)$ e portanto $\pi^{k}(e)=$ $g^{k}(e)$

Como isso estamos em condiçôes de verificar que podemos construir uma sequência de funçōes com as propriedades desejadas

\section{TEOREMA.8.1.19}

Seja $k \in \mathbb{N}$ uma iteração do método de incrementos e suponhamos que

$$
\begin{aligned}
& \exists \pi^{k}: \mathbb{R}^{p} \rightarrow \mathbb{R} \pi^{k}(u)=\min _{e i n C^{k}}\left\{g^{k}(e)+\Delta^{k}(u-e)\right\} \\
& \pi^{k}(.) \in \Omega^{k-1}
\end{aligned}
$$


$\operatorname{com} \Delta^{k}()=.\alpha^{k}\left\|_{\|}\right\|_{1}$ para algum $\alpha^{k} \in \mathbb{R}$

Entäo $\exists \pi^{k+1}(.) \in \Omega^{k}$ definida por

$\pi^{k+1}(u)=\min _{e \in C^{k+1}}\left\{g^{k+1}(e)+\Delta^{k+1}(u-e)\right\}$

onde

$\Delta^{k+1}()=.\alpha^{k+1}\|\cdot\|_{1} \alpha^{k+\triangleq}=\alpha^{k}+e^{k} C^{k+1}=c^{k} \cup\left\{e^{k}\right\}$ p algum $e^{k} \in O^{k}$

\section{Demonstração}

Considere a construção da função $\pi($.$) conforme proposto pelo lema.8.1.12.$
a) $\exists \bar{e} \in O^{k} \mid V(\bar{e})=\emptyset$

Neste caso de fato 8.1.18 temos que $\pi^{k}(\bar{e})=g^{k}(\bar{e})$ e tomando-se $C^{k+1}=C^{k} \cup \bar{e}$ segue a afirmaçäo.

b) $\forall e \in O^{k} V(e)=\emptyset$

Considere a função $\pi($.$) proposta em 8.1.12. Se para o elemento$ escolhido $e^{k} \pi\left(e^{k}\right)=g^{k}\left(e^{k}\right)$, tomamos

$$
\begin{aligned}
& \pi^{k+1}(u)=\min _{e \in C}{ }^{k+1}\left\{g^{k+1}(e)+\Delta^{k+1}(u-e)\right\} \text { onde } \\
& C^{k+1}=C^{k} \cup\left\{e^{k}\right\}\left(g^{k+1}(e)=g^{k}(e) \forall e \in C^{k}\right) \text { Se } \pi\left(e^{k}\right)<g^{k}\left(e^{k}\right) ; \\
& \text { como de 8.1.18 } \\
& g^{k}\left(e^{k}\right)=g^{k}\left(e_{1}^{k}\right)+\Delta^{k+1}\left(e^{k}-e_{1}^{k}\right) \\
& \text { temos que } V^{\prime}(e)=\left\{e \in C^{k} \mid g^{k}(e)>g^{k}\left(e_{1}\right)+\Delta\left(e-e_{1}\right)\right\} \# V^{\prime}(e)<
\end{aligned}
$$
$\# V(e)<\infty$ (pois $V(e) \subset C^{k}$ finito)

Repetindo-se o processo construtivo temos que ao final de um número finito de passos esse conjunto será vazio e podemos então determinar $\pi^{k+1}($.$) Note que o processo não altera as demais propriedades das funções$ $\pi($.$) de forma que ao final de um número finito de passos o proceeso termina$ $\operatorname{com} \pi^{k+1}(.) \in \Omega^{k}$

Podemos assim construir uma sequência de funçoes aplicáveis ao método de incrementos subaditivos, e cuja obtenção é trivial. Nesse caso, dada uma função $\pi^{k}($.$) , a "taxa de crescimento" que não viola as pro-$ priedades necessárias para que o método termine é bem determinada e não 
leva em consideração a a proximidade do "alvo", de tal forma que pode ser necessário pesquisarmos todos os elementos do grupo até que possamos determinar uma solução ótima do problema.

\section{EXEMPLO 8.1.20}

Considere o seguinte problema :

$\min _{1}+x_{2}+x_{3}+x_{4}+x_{5}+x_{6}+x_{7}+x_{8}$

sa $\sum_{j=1}^{9} x_{j} \equiv 0.1$

$x_{j} \geqq 0$ inteiro, $\forall j 1 \leqq j \leq 9$

Resolvendo-o pelo método de incrementos, seguindo o processo de construtivo proposto, temos $N=\cup_{j=9}^{9}\{j / 10\} \cup\{0\}$

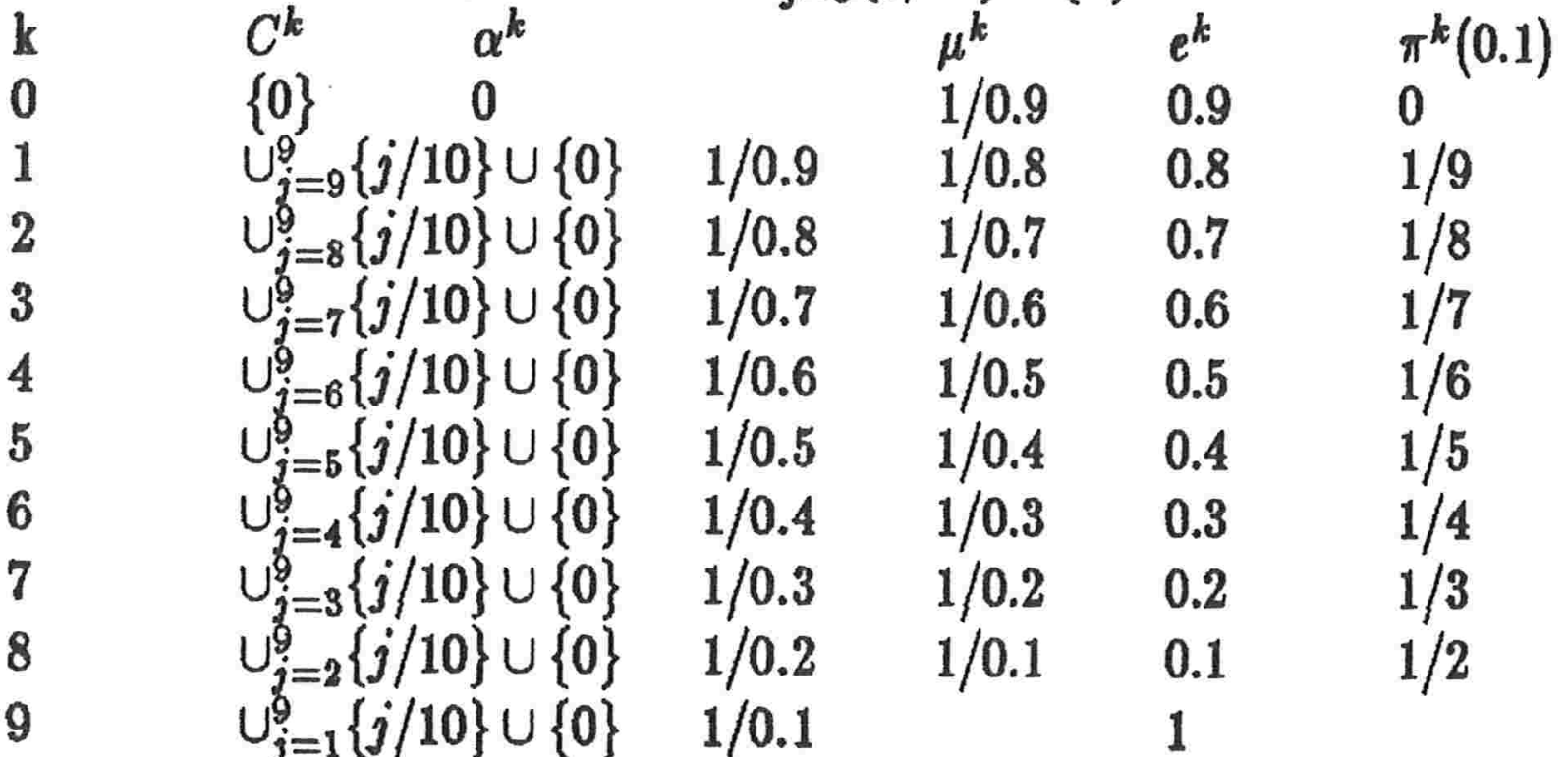

Nesté caso para que o elemento 0.1 fosse fechado precisamos fechar todos os elementos do grupo.

Esse tipo de situação ocorre por estarmos considerando apenas a informação contida em $O_{L}^{k} \cup C_{L}^{k}$ sem nos preocupar com a "proximidade do alvo", $f^{0}$. Uma possível aceleração do algoritmo deve ser obtida a incluirmos no processo construtivo alguma informação desse tipo.

\subsection{A CONSTRUÇÃO DE FUNÇÕES INFORMADAS}

Uma função subaditiva fortemente relacionada com o problema de grupo é a função perturbação $v^{R}($.$) de sua relaxação simples. Assim, a$ 
utilização de $\pi()=.v^{R}($.$) seria adequada a nossos objetivos se pudessemos$ assegurar que $\pi(e)=g^{k}(e) \forall e \in C^{k}$, o que em geral não ocorre.

Para que não tivessemos este tipo de problema, poderiamos "erguer" o valor de $\pi($.$) nesses pontos, eventualmente perdendo a subaditivi-$ dade assegurada por $v^{R}($.$) .$

\section{FATO 8.2.1}

Seja $k \in \mathbb{N}$ uma iteração do algoritmo de incrementos relaxado e $\pi($.$) definida por:$

$\pi: \mathbb{R}^{p} \rightarrow \overline{\mathbf{R}}$

$$
\pi(y)= \begin{cases}v^{R}(y) & \text { y } \not \equiv \quad j \forall j \in C_{L}^{k} \cup\left\{e^{k}\right\} \\ g^{k}(j) & \exists j \in C_{L}^{k} \cup\left\{e^{k}\right\}: y \equiv j\end{cases}
$$

, onde $e^{k} \in O^{k}: g^{k}\left(e^{k}\right)=v(e)$.

Então $\pi(.) \in \Omega^{k}$.

Demonstração

i.) $\pi(.) \in W_{G}$

Seja $x \geqq 0, x \in \mathbb{Z}^{q}$ Se $\exists j \in C_{L}^{k} \cup\left\{e^{k}\right\} \mid F x \equiv j$, então

$\pi(F x)=g^{k}(F x)=v(F x) \leq\langle d, x\rangle$

Caso contrário, $\pi(F x)=v^{R}(F x) \leqq v(F x) \leqq\langle d, x\rangle$

ii.) $\forall e \in O_{L}^{k} \pi(e) \leqq g^{k}(e)=v(e)$

Trivial

iii.) $\forall e \in C_{L}^{k} \cup\left\{e^{k}\right\} \pi(e)=g^{k}(e)$

Imediato por construção.

De (i), (ii) e (iii) segue a afirmação

Nosso problema passa então a ser a determinação do vetor $e^{k} \in O_{L}^{k} \backslash C_{L}^{k}$ para o qual tenhamos $g^{k}\left(e^{k}\right)=v(e)$ (o qual sabemos sempre existe). $O$ algoritmo $A^{*}$ ao utilizar subestimadores consistentes, escolhia a cada iteração, um elemento aberto para o qual se dispunha do 
valor ótimo v(e).Utilizando - se aquele critério de escolha para fechamento temos:

\section{PROPOSIÇÃO 8.2.2}

Nas condiçóes do fato anterior

Se $e^{k} \in \arg \min _{e \in O_{L}^{k} \backslash C_{L}^{k}}\left\{g^{k}(e)+v^{R}\left(f^{0}-e\right)\right\}$

então

$$
\pi(.) \in \Omega^{k}
$$

\section{Demonstração}

Basta verificar que $g^{k}\left(e^{k}\right)=v\left(e^{k}\right)$.

Suponhamos que $g^{k}\left(e^{k}\right)>v\left(e^{k}\right)$.

Pela proposição 7.1.12 $\exists \bar{x} \in S(\hat{x}) \hat{x} \in \arg \min _{x \in X_{e^{k}}}\langle d, x\rangle \mid$ $g^{k}(F \bar{x})=v(F \bar{x}) F \bar{x} \in O_{L}^{k} \backslash C_{L}^{k}$

Seja $\bar{e}=F \bar{x}$ entẫo,

$g^{k}(\bar{e})+v^{R}\left(f^{0}-\bar{e}\right) \leqq g^{k}(\bar{e})+v^{R}\left(f^{0}-\bar{e}\right)+v\left(e^{k}-\bar{e}\right)$

Como $\bar{x} \in S(\hat{x})$, vem que $v\left(e^{\bar{x}}\right)=v(\overline{\bar{F}} \hat{x})=v(\overline{\bar{e}})+v\left(e^{k}-\bar{e}\right)$ $v^{R}\left(f^{0}-e^{k}\right)$

e, portanto, $g^{k}(\bar{e})+v^{R}\left(f^{0}-\bar{e}\right) \leqq v\left(e^{k}\right)+v^{R}\left(f^{0}-\bar{e}\right)<g^{k}\left(e^{k}\right)+$

contrariando a escolha de $e^{t}$ para fechamento .

Dessa forma podemos utilizar uma sequência de funções construida iterativamente de acordo com o fato 8.2.1, escolhendo a cada passo para inclusão em $C_{L}^{k}, 0$ elemento $e^{k}$ determinado como na proposição anterior, reproduzindo o critério de escolha para fechamento de $A^{*}$.

Este critério tem a desvantagem de possuir complexidade alta pois, a determinação de $e^{k}$ pressupóe a resolução de \# $O_{L}^{k}$ subproblemas,

$\min _{x \in X_{f^{0}-\varepsilon}^{r}}<d, x>$.A fim de reduzir este número, podemos adotar outras funçốes em substituição a $v^{R}($.$) .$ 


$$
\Gamma=\left\{u \in \mathbb{R}^{p} \mid F^{t} u \leqq d u \geqq 0\right\} \neq \emptyset
$$

Demonstração

Trivial, pois por hipótese $d \geqq 0$.

\section{FATO 8.2.4}

$\forall e \in N, X_{e}^{r} \neq \emptyset$

Demonstração

Trivial

\section{LEMA 8.2.5}

$\forall e \in N, \max _{u \in \Gamma}<e, u>\in \mathbb{R}$

Demonstração

Trivial por dualidade.

Nessas condições podemos construir a seguinte sequência de funકões:

\section{PROPOSIÇÃO 8.2.6}

Seja $V \in N, V=\left\{e^{1}, e^{2}, \ldots, e^{m}\right\}$, e $\Delta($.$) definida por:$

$\left.\Delta: \mathbb{R}^{\mathrm{p}} \rightarrow \mathbb{R} \Delta(y)=\max _{1} \leqq i \leqq m<u^{i}, y\right\rangle u^{i} \in \arg \max _{u \in \Gamma}\left\langle e^{i}, u\right\rangle$

Então $\Delta($.$) é subaditiva.$ 
Demonstração

$$
\begin{aligned}
& \forall\left(y_{1}, y_{2}\right) \in \mathbb{R}^{\mathrm{P} x} \mathbb{R}^{\mathrm{p}} \\
& \Delta\left(y_{1}+y_{2}\right)=\max _{1 \leqq i \leqq m}\left\{<u^{i}, y_{1}+y_{2}>\right\} \\
& \leqq \max _{1 \leqq i \leqq m}\left\{<u^{i}, y_{1}>\right\}+\max _{1 \leqq i \leqq m}\left\{<u^{i}, y_{2}>\right\} \\
& =\Delta\left(y_{1}\right)+\Delta\left(y_{2}\right)
\end{aligned}
$$

A aplicação desta função ao algoritmo de incrementos é imediata:

\section{NOTAÇÃO 8.2.7}

Para o algoritmo de incrementos relaxado denotamos por $\left\{\Delta^{k}(.)\right\}_{k \in \mathbb{N}}$ à sequência de funçōes

$$
\begin{gathered}
\Delta^{k}: \mathbb{R}^{p} \rightarrow \mathbb{R} \\
\Delta^{k}(y)=\max _{1 \leqq i \leqq k}\left\langle u^{i}, y>\right.
\end{gathered}
$$

, onde, $u^{i} \in \arg \max _{u \in \Gamma}\left\langle e^{i}, u\right\rangle$

$e^{i} \in C^{i+1} \backslash C^{i}, \forall i \in\{1,2, \ldots, k-1\}$

\section{PROPOSIÇÃO 8.2.8} definida por:

Considere o algoritmo de incrementos relaxado, e seja $\left\{\pi^{k}(.)\right\}_{k \in \mathbb{N}}$ $\pi^{0}()=$.

$$
\pi^{k}(y)= \begin{cases}\Delta^{k}(y) & y \neq j \forall j \in C_{L}^{k} \cup\left\{e^{k}\right\} \\ g^{k}(j) & \exists j \in C_{L}^{k} \cup\left\{e^{k}\right\}: y \equiv j\end{cases}
$$

onde,

8.2 .7

$e^{k} \in \arg \min _{e \in O_{L}^{k} \backslash C_{L}^{k}}\left\{g^{k}(e)+\Delta^{k}\left(f^{0}-e\right)\right\} \Delta^{k}($.$) conforme a notacão$ Então, $\forall k \in \mathbb{N}, \pi^{\mathbf{k}}(.) \in \Omega^{\mathbf{k}}$. 
Demonstração

i.) $\forall e \in C_{L}^{k} \cup\left\{e^{k}\right\} \pi^{k}(e)=g^{k}(e)$

Da subaditividade de $\Delta^{k}($.$) que \forall e \in C_{L}^{k} g^{k}(e)=v\left(e^{k}\right)$

ii) $\forall e \in O_{L}^{k} \backslash C_{L}^{k} e \neq e^{k} \pi^{k}(e) \leqq g^{k}(e)$

De fato

$$
\forall e \in O_{L}^{k} \backslash C_{L}^{k} e \neq e^{k} \pi^{k}(e)=\Delta(e)=\max _{1 \leqq i \leqq k}\left\langle u^{i}, e\right\rangle
$$

Como $u^{i} \in \Gamma \quad \forall i 1 \leqq i \leqq k, \Delta^{k}(e) \leqq \max _{u \in \Gamma}\langle e, u\rangle=\inf _{x \in X_{e}^{R}}\langle d, x\rangle$ $\leqq \Gamma(e) \leqq g^{k}(e)$

iii) $\pi^{k}(.) \in W_{g}$

Trivial pois

$\forall x \in \mathbb{Z}^{q} x>0 \pi(F x) \leqq v(F x) \leqq\langle d, x\rangle$

De (i),(ii),(iii) segue a afirmação

Assim podemos construir uma sequência de funções aplicáveis ao método de incrementos para o qual vale

\section{LEMA 8.2.9}

Se 0 algoritmo de incrementos utiliza a sequência de funçōes

$\left\{\pi^{k}(.)\right\}_{k \in \mathbb{N}}$ construida na proposição anterior, escolhendo para fechamento a cada iteração, o elemento $e^{k} \in N$, então:

$\forall y \in \mathbb{R}^{\mathrm{p}}\left\{\pi^{\mathrm{k}}(\mathrm{y})\right\}_{\mathrm{k} \in \mathbb{N}}$ é não decrescente.

Demonstração

Seja $y \in \mathbb{R}^{\mathrm{p}}, \mathrm{k} \in \mathbb{N}$

Se $\pi^{k}(y)=\Delta^{k}(y)$ e $\pi^{k+1}(y)=\Delta^{k+1}(y)$

trivialmente

$\Delta^{k}(y)=\max _{1 \leqq j \leqq k}\left\langle u^{i}, y\right\rangle \leqq \max _{1 \leqq j \leqq k+1}\left\langle u^{i}, y\right\rangle=$ 
$=\Delta^{k+1}(y)$

Se $\pi^{k}(y)=\Delta^{k}(y)$ e $\pi^{k+1}(y)=g^{k+1}(y)$ então

$\pi^{k}(y)=\Delta^{k}(y) \leqq v(y) \leqq g^{k}(y)=\pi^{k+1}(y)$

Se $\pi^{k}(y)=g^{k}(y)$ e $\pi^{k+1}(y)=g^{k}(y)$ a afirmação é imediata

Com a utilização de funções $\Delta^{k}($.$) e o do critério de escolha de$ elemento para fechamento da proposição 8.2.9, reduzimos significativamente - número de subproblemas a serem resolvidos.Para obter $\Delta^{k+1}($.$) , devemos$ determinar apenas $u^{k+1} \in \arg \max _{x \in \Gamma}$ pois já conhecemos $u^{1}, u^{2}, \ldots, u^{k}$.

Aliás a obtenção de tal elemento é bastante simples, pois o conjunto de pontos viáveis, $\Gamma$,é invariante com as iterações.

\subsection{A RELAÇÄO ENTRE O MÉTODO DE INCREMENTOS E}

Duas abordagens podem ser consideradas ao compararmos os dois algoritmos vistos.Primeiramente podemos considerar o algoritmo de incrementos subaditivo como um processo que gera heurísticas consistentes,através da construção de funções viáveis no dual subaditivo do problema de grupos.Como a cada passo gera-se funçóes cada vez mais altas,estamos construindo uma sequência de estimadores que variam com as iteraçöes.

Vimos também que $A^{*}$ gerava sequências de funçôes viáveis no dual geral do problema de grupos (em alguns casos até mesmo no dual subaditivo), que podiam ser utilizadas pelo método de incrementos e poderia também,ser interpretado como um procedimento de geração de funções.

A questão então passa a ser a possibilidade de que os dois algoritmos possam ser um só.Intuitivamente se tomassemos a sequência de funções construída na proposição 8.2 .9 ,reproduziríamos o algoritmo $A^{*}$,porém devido ao critério de abertura de nós isso não ocorre.

\section{EXEMPLO 8.3.1}

Considere o seguinte problema 
$\min 2 x_{1}+3 x_{2}$

s.a. $0.1 x_{1}+0.2 x_{2}=0.3$

$x_{1} \geqq 0 x_{2} \geqq 0 x_{1}, x_{2}$ inteiros

$\mathrm{O}$ grafo correspondente a este problema é

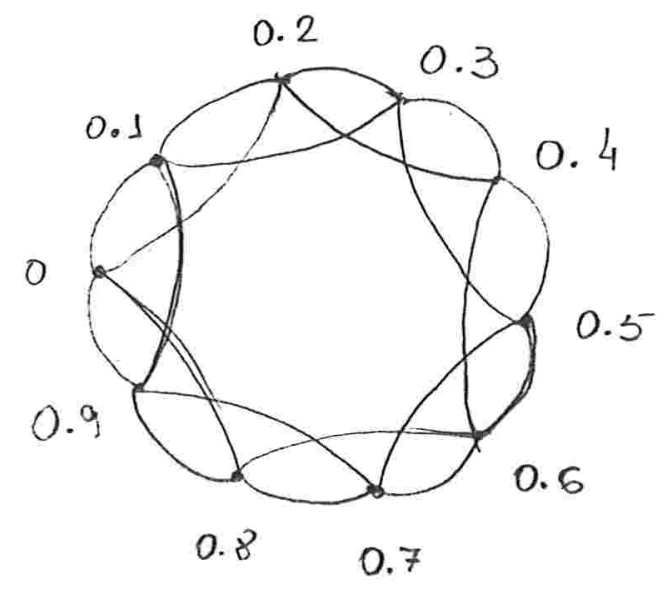

Aplicando-se $o$ algoritmo * $A^{*}$ a este grafo,com a sequência de heurísticas $\left\{h^{k}(.)\right\}_{k \in \mathbb{N}}, h^{k}(j)=\Delta^{k}\left(f^{0}-j\right)$, e considerando-se a aplicação do método de incrementos a este problema teríamos

$\begin{array}{lllll}k & C^{k} & O^{k} & C_{L}^{k} & O \\ 1 & \{0\} & \{0.1,0.2\} & \{0\} & \{0.1,0.2\} \\ 2 & \{0,0.1\} & \{0.2,0.3,0.4\} & \{0.0 .1\} & \{0.2\} \\ 3 & \{0,0.1,0.3\} & \{0.2,0.4\} & \{0,0.1,0.2\} & \{0.3\}\end{array}$

Neste caso o algoritmo $A^{*}$ termina em um número de iteraçôes menor que no caso do método de incrementos, sendo que podemos construir exemplos onde esta situação se reverte.

É claro que um dos fatores que causam essa diferença no desempenho dos dois algoritmos é o critério de abertura de nós, distinto nos dois casos. Assim, poderiamos considerar

\section{DEFINIÇÃO 8.3.2}

Chamamos de algoritmo de incrementos modificados 2.0 obtido a partir do algoritmo de incrementos relaxado com um critério de abertura de 
elemento de $A^{*}$,isto é, a cada $j_{r}$ escolhido para fechamento,são considerados para possível inclusão em $O_{L}^{k+1}$ apenas os sucessores de $j_{r}$,ou seja, $y \in N$ : $y \equiv j_{r}+F^{l} l \in\{1, \ldots, q\}$

Não verificaremos a validade do método assim obtido pois repetiríamos resultados já exaustivamente estudados.Parece-nos que segundo esta definição tem-se

\section{RESULTADO 8.3.3}

Considere o algoritmo $A^{*}$, a 0 utilizar uma sequência de estimadores $\left\{h^{k}(.)\right\}_{k \in \mathbb{N}}$,consistentes,admissíveis e não decrescentes com as iteraçốes.

Então sempre é possível reproduzí-lo através do algoritmo modifcado,isto é, $\exists\left\{\pi^{k}(.)\right\}_{k \in \mathbb{N}}$ tal que o utilizá-la para este algoritmo

$\forall k \leqq\left\{k_{1}, k_{2}\right\} O_{L}^{k}=O^{k}$ e $C_{L}^{k}=C^{k}$

$k_{1}$ e $k_{2}$ as iterações nas quais os algoritmos terminam.

Para que verificassemos esse resultado,seria necessário provarmos a finitude do algoritmo.Porém parece-nos que para tanto basta tomar$\operatorname{mos}\{\pi k(.)\}_{k \in \mathbb{N}}$ a sequência construida segundo a proposição 6.2.12 com o mesmo critério de escolha (isto é,fechando-se na iteração $\mathrm{k}$ o elemento $j k$ )

\subsection{CONCLUSÃO}

Neste capitulo apresentamos alguns processos construtivos de funsôes aplicáveis ao algoritmo de incrementos que,além de nos permitir fazer algumas identificaçōes entre esses algoritmos e $A^{*}$ aplicado a (PG),parecem conduzir a bons resultados computacionais.

Não é possível determinar a priori, qual dos algoritmos possue melhor desempenho na resolução de um dado problema, porém a utilização conjunta dos critérios de abertura de nós e de parada dos dois algoritmos parece fornecer algoritmos eficientes. 


\section{CONCLUSÃO}

Os capítulos 1,2 e 3 deste trabalho não apresentam resultados originais,tendo uma função de certa forma didática.

No capítulo 4,generalizamos alguns resultados de Pearl [Pe83] ao utilizar heurísticas viáveis com as iteraçóes no algoritmo $A^{*}$. e apresentamos uma relação entre subaditividade e heurísticas consistentes.

Não é usual na literatura o estudo detalhado do problema de grupos conforme apresentado no capítulo 5 ,assim como a utilização de $A^{*}$ para a sua resolução,sendo a principal função do capítulo 6 é permitir uma caracterização deste algoritmo como um processo de geração de funções viáveis no dual do problema de grupos.

Os métodos de incrementos vistos no capítulo 7 baseiam-se em trabalhos de Johnson,sendo que este autor restringe ao estudo do caso subaditivo, não tratando do método do incremento relaxado que apresentamos. 
Quanto ao capítulo 8,procuramos apresentar dois processos de construção de funçōes subaditivas,sendo o primeiro deles uma particularização do trabalho de Johnson,enquanto que o segundo é de nossa autoria.

Alguns pontos ficam ainda em aberto. Quanto a aspectos computacionais parece nos que algoritmos que englobem conceitos de $A^{*}$ e do método de incremento conduzem a bons resultados na resolução do problema de grupos e de porgramação inteira. 
Apêndice 1

\section{Alguns resultados sobre grafos}

A função deste apêndice e fixar a notação e mencionar os principais resultados sobre teoria de grafos utilizadas no trabalho. Como referência básica utilizamos [BM76] e [Ha69]

\section{DEFINIÇÃO A1.1}

Um GRAFO ORIENTADO, ou simplesmente, GRAFO é uma terna ordenada $\left(N, E, \xi_{G}().\right)$, onde $\mathrm{N}$ é um conjunto enumerável e não vazio de elementos chamados nós, $\mathrm{E}$ um conjunto disjunto de $\mathrm{N}$ de arcos e $\xi_{G}($. uma função chamada função de incidência, $\xi_{G}: E \rightarrow N \times N$,que associa 
a cada arco ,um par ordenado de nós,não necessariamente distintos.Por simplicidade, denotaremos tal grafo por $G \equiv(N, E)$.

Diremos que $G \equiv(N, E)$ é finito, se $\# N<\infty$ e \# $E<\infty$.

Da mesma forma definimos um grafo não orientado como uma terna $\left(N, E, \xi_{G}().\right)$ sendo neste caso, $\mathrm{E}$ um conjunto de arestas,e $\xi_{G}($.$) definida$ sobre pares não ordenados de nós.

\section{DEFINIÇÃO A1.2}

Dado um grafo $G \equiv(N, E)$, chamamos de OPERADOR SUCESSOR de $G$ a aplicação ponto-conjunto :

$$
\begin{gathered}
\text { suc }: N \rightarrow 2^{N} \\
\operatorname{suc}(n)=\{j \in N \mid(n, j) \in E\}
\end{gathered}
$$

Os elementos de suc(n) são chamados nós adjacentes a $\mathrm{n}$.

\section{DEFINIÇÃO A1.3}

Dado um grafo $G \equiv(N, E)$, e um nó $n \in N$, definimos o grau de n, $\delta(n), \operatorname{como} \delta(n)=\#\{e \in E \mid \exists j \in N: e=(n, j)$ ou $e=(j, e)\}$

\section{DEFINIÇÃO A1.4}

Dado um grafo orientado $G \equiv(N, E)$, e $\left(v_{0}, v_{r}\right) \in N \times N$, chamamos de passeio entre $v_{0}$ e $v_{r}$ a qualquer sequência finita

$$
W=\left(v_{0}, a_{1}, v_{1}, \ldots, a_{r}, v_{r}\right)
$$

onde,

$$
\begin{aligned}
& \forall i \in\{1,2, \ldots, r\} v_{i} \in N, a_{i} \in E \\
& \text { e } a_{i}=\left(v_{i-1}, v_{i}\right) .
\end{aligned}
$$

Diremos nesse caso que $\mathrm{W}$ possui comprimento $\mathrm{k}$. 
No caso em que a função de incidência do grafo, $\xi_{G}($.$) , e injetora, e$ $\forall v \in N, e=(v, v) \xi_{G}(e) \neq(v, v)$ (isto é, o grafo não possue laços),podemos denotar um passeio simplesmente através de seus vértices.Em geral, diremos que um arco $a_{j}$ pertence ao caminho W se, $\exists j \mid a=a_{j}$.

\section{DEFINIÇÄO A1.5}

Dado um grafo $G \equiv(N, E)$, chamamos de semi-passeio entre $v_{0} \mathrm{e}$ $v_{r}$ a qualquer sequência finita

$$
W=\left(v_{0}, a_{1}, v_{1}, \ldots, a_{r}, v_{r}\right)
$$

onde $v_{0} \in N$ e $\forall i \in\{1,2, \ldots r\} v_{i} \in N \mathrm{e}$

$a_{i}=\left(v_{i-1}, v_{i}\right)$ ou $a_{i}=\left(v_{i}, v_{i-1}\right){ }^{*}$

\section{DEFINIÇÃO A1.6}

Chamamos de caminho entre $v_{0}$ e $v_{r}$ a qualquer passeio tal que seus nós são distintos.Diremos ainda que um passeio é um circuito entre $v_{0} \mathrm{e} v_{r}$ se $v_{0}=v_{r}$, e todos os demais nós são distintos. Analogamente ao caso de passeios, define-se semi-circuitos em grafos.

\section{DEFINIÇÄO A1.7}

Diremos que o grafo $G \equiv(N, E)$ é aciclico se não possuir semicircuitos.

\section{DEFINIÇÃO A1.8}

Dado um grafo $G \equiv(N, E)$, e $(i, j) \in N \times N$, dizemos que j é atingível a partir de i se existe um passeio entre i $\mathrm{e} j$.

* Note que esse conceito corresponde a idéia de eliminarmos a orientação do grafo, e considerarmos "passeios" no grafo não orientado então obtido 


\section{DEFINIÇÃO A1.9}

Dado um grafo $G \equiv(N, E)$, e $(i, j) \in N \mathrm{x} N$, dizemos que G e conexo se $\forall(i, j) \in N \times N$ existe um semi-passeio entre i e j.

Diremos ainda que $\mathrm{G}$ e fortemente conexo se $\forall(i, j) \in N \times N$ existe um passeio entre i e j.

Nessas condições valem os seguintes resultados:

\section{PROPOSIÇÃO A1.10}

Se $G \equiv(N, E)$ é um grafo finito, então o número de caminhos distintos entre qualquer par de nós $(\mathrm{i}, \mathrm{j})$ de $\mathrm{G}$ é finito.

\section{LEMA A1.11}

Dado um grafo $G \equiv(N, E)$,

se $\bar{\delta}=\min _{n \in N} \delta(n) \geqq 2$, entấo G contém um semi-circuito.

\section{DEFINIÇÄO A1.12}

Dado um grafo $G \equiv(N, E)$, dizemos que $\mathrm{G}$ é uma semi-árvore se é fortemente conexo e não possui semi-circuitos (isto é, e aciclico).G sera uma semi-árvore se for conexo e aciclico.

\section{TEOREMA A1.13} aciclico

Um grafo $G \equiv(N, E)$ é uma árvore $\Longleftrightarrow \# E=\# N+1$ e $\mathrm{G}$ é

\section{PROPOSIÇÃO A1.14}

Se o grafo $G \equiv(N, E)$ é uma semi-árvore então possui pelo menos dois nós pendentes, isto é, tal que $\delta(n)=1$. 


\section{PROPOSIÇÃO A1.15}

Se $G \equiv(N, E)$ é uma semi-árvore e possui apenas dois nós pendentes, então e m semi-caminho.

Ao associarmos custos aos arcos de um grafo, temos:

\section{DEFINIÇÃO A1.16}

Seja $G \equiv(N, E)$ um grafo com custos associados aos arcos,isto é, tal que $\exists c: E \rightarrow \mathbb{R}$. Dado um passeio $W=\left(v_{0}, v_{1}, \ldots v_{r}\right)$ entre $v_{0} \mathrm{e} v_{r}$, definimos o custo de $W$ por :

$$
c(W)=\sum_{I=1}^{r} c\left(v_{i-1}, v_{i}\right)
$$

Um resultado bastante interessante é o princípio de otimilidade :

\section{TEOREMA A1.17[Go78]}

Considere $G \equiv(N, E)$, um grafo com custos associados as arestas e $k: N \times N \rightarrow \overline{\mathbb{R}} k(i, j)=\inf \{c(P) \mid \mathrm{P}$ é um caminho entre i e j $\}$

Sendo $P_{1 r}=\left(n_{1}, n_{2}, \ldots, n_{r}\right)$, um passeio entre $i_{1}$ e $i_{r}$,vale:
Se $c\left(P_{i j}\right)=k\left(n_{1}, n_{r}\right) P_{i j}=(n 1, n 2, \ldots n r)$, então $\forall(i, j) 1 \leqq i<j \leqq r c\left(P_{i j}\right)=$
$k(i, j)$.

Outros resultados a respeito de grafos e aplicaçôes podem ser encontrados nas referências já citadas. 
Apêndice 2

\section{Dualidade Generalizada em Progrmação Inteira}

O trabalho de Tind e Wolsey [TW81], apresenta resultados sobre dualidade generalizada, aplicáveis a problemas de programação matemática em geral. Verificaremos aqui alguns resultados relacionados com problemas lineares.

DEFINIÇÄO A.2.1

Dado o problema primal $(P) \inf _{x \in X} f(x)$

$$
\begin{gathered}
X=\left\{x \in X^{0} \mid g(x)=b\right\} \\
X^{0} \subset \mathbb{R}^{q}, g: \mathbb{R}^{q} \rightarrow \mathbb{R}^{p} f: \mathbb{R}^{q} \rightarrow \mathbb{R}
\end{gathered}
$$


definimos o DUAL GERAL DE $(P)$ por

$$
\begin{gathered}
(D P) \sup _{\phi(.) \in U} \phi(b) \\
U=\left\{\phi(.) \in \Im \mid \phi(g(x)) \leqq f(x) \forall x \in X^{0}\right\}, \\
\Im \subset\left\{\xi: \mathbb{R}^{\mathrm{P}} \rightarrow \overline{\mathbb{R}}\right\}
\end{gathered}
$$

TEOREMA A.2.2

$$
\sup _{\phi(.) \in U} \phi(b) \leqq \inf _{x \in X} f(x)
$$

Demonstração

Se $U=\emptyset$ ou $X=\emptyset$ a afirmação segue trivialmente.

Caso contrário,sejam

$x^{*} \in X$ e $\phi^{*}(.) \in U$.

Claramente,

$$
\phi^{*}(b)=\phi^{*}\left(g\left(x^{*}\right)\right) \leqq f\left(x^{*}\right) .
$$

Uma função que nos será útil é :

\section{DEFINIÇÄO A.2.3}

A função perturbação, ( ou função valor ótimo) v(.) associada ao problema $(P)$ é definida por :

$$
v: \mathbb{R}^{\mathrm{P}} \rightarrow \overline{\mathbb{R}}
$$




$$
\begin{gathered}
v(y)=\inf _{x \in X_{y}} f(x) \\
X_{y}=\left\{x \in X^{0} \mid g(x)=y\right\}
\end{gathered}
$$

LEMA A.2.4

Se $v(.) \in \Im$, então $v(.) \in U$

Demonstração

Basta observar que $\forall x \in X^{0}, f(x) \leqq v(g(x))$.

TEOREMA A.2.5 (Teorema forte de dualidade)

Se $v(.) \in \Im$ então, $\sup _{\phi(.) \in U} \phi(b)=\inf _{x \in X} f(x)$

Demonstração

Se $v(.) \in \Im \subset U$,

$v(b) \leqq \sup _{\phi(.)} \phi(b) \leqq \inf _{x \in X} f(x)$

Portanto,

$v(b)=\sup _{\phi(.)} \phi(b) \inf _{x \in X} f(x)=v(b)$.

0 conjunto $\Im$ sobre o qual as funçōes $\phi($.$) são definidas varia de$ acordo com o problema considerado.

\section{DEFINIÇÃO A.2.6}

A função $\varrho: W \rightarrow \mathbb{R}\left(W^{-} \subset \mathbb{R}^{p}\right)$ é dita subaditiva em $W$ se, 


$$
\begin{gathered}
\forall(\alpha, \beta) \in W \times W: \alpha+\beta \in W \\
\varrho(\alpha+\beta) \leqq \varrho(\alpha)+\varrho(\beta)
\end{gathered}
$$

\section{DEFINIÇÃO A.2.7}

Chamamos de problema inteiro, (PI), a

$$
\begin{gathered}
\inf _{x \in X_{P I}} f(x) \\
X_{P I}=\left\{x \in X_{P I}^{0} \mid A x=b\right\} \\
X_{P I}^{0}=\left\{x \in \mathbb{Z}^{q} \mid x \geqq 0\right\}, A \in \mathbb{R}^{\mathrm{p} \times \mathrm{q}}
\end{gathered}
$$

\section{LEMA A.2.8}

Se para o problema inteiro (PI) a função $f($.$) é subaditiva então$ sua função perturbação $v($.$) é subaditiva .$

Demonstração

Seja $\left(y_{1}, y_{2}\right) \in \mathbb{R} x \mathbb{R}$ tais que os problemas

$P_{y_{1}}=\inf _{x \in X_{y_{1}}} f(x)$ e $P_{y_{3}}=\inf _{x \in X_{y_{3}}} f(x)$

possuam solução ótima, $x_{1}$ e $x_{2}$ respectivamente.

Como, $X_{y_{1}}+X_{y_{2}} \subset X_{y_{1}+y_{2}}, x_{1}+x_{2}$ é viável em

$P_{y_{1}+y_{2}}=\inf _{x \in X_{y_{1}+y_{2}}} f(x)$

Mas $f($.$) é subaditiva, e portanto$

$f\left(x_{1}+x_{2}\right) \leqq f\left(x_{1}\right)+f\left(x_{2}\right) \leqq v\left(y_{1}\right)+v\left(y_{2}\right)$.

Logo,

$v\left(y_{1}+y_{2}\right)=\inf _{x \in X_{y_{1}+z_{2}}} f(x) \leqq f\left(x_{1}+x_{2}\right) \leqq v\left(y_{1}\right)+v\left(y_{2}\right)$ análoga.

Os casos onde ocorre ilimitação ou inviabilidade seguem de maneira 
Nessas condições,

\section{TEOREMA A2.9}

Se $\Im^{*}=\Im_{\text {sub }}=\left\{\psi: \mathbb{R}^{p} \rightarrow \mathbb{R} \mid \psi(\right.$.$\left.) subaditiva \right\}$,

vale o teorema forte de dualidade para (PI)

Demonstração

Do lema anterior , $v(.) \in \Im$, e portanto do teorema A2.5 segue a afirmação.

Como caso particular, temos:

\section{TEOREMA A2.10}

Nas condições do teorema anterior, o dual de (PI) é equivalente a $\inf _{\phi(.) \in U_{\text {sub }}} \phi(b)$,

$U_{s u b}=\left\{\phi(.) \in \Im_{\text {sub }} \mid \phi\left(A^{j}\right) \leqq c_{j} \forall j 1 \leqq j \leqq q\right\}$ $A^{j}$ a j-ésima coluna da matriz $\mathrm{A}$.

Demonstração

Basta verificar que tomando-se $\Im=\Im_{s u b}$, $U_{\text {sub }}=\left\{\phi(.) \in \Im_{\text {sub }} \mid \phi\left(A^{j}\right) \leqq c_{j} \forall j 1 \leqq j \leqq q\right\}=$

$=\left\{\phi(.) \in \Im_{s u b} \mid \phi(A x) \leqq\left\langle c, x>\forall x \in \bar{X}_{P I}^{0}\right\}=U\right.$

o que segue trivialmente da subaditividade das funçóes $\phi($.$) .$

Nestas condições, folgas complementares são dadas por: 
Considere o problema linear inteiro e seu dual geral, para o caso em que $\Im=\Im_{s u b}$.Sendo $x \in X_{P I}$ e $\phi(.) \in U_{s u b}$, tem-se:

$$
<c, x>=\phi(b) \Leftrightarrow
$$

i.)

$$
\sum_{j=1}^{q} x_{j} \phi\left(A^{j}\right)=\sum_{j=1}^{q} x_{j} c_{j}
$$

ii.)

$$
\phi(A x)=\langle c, x>
$$

Demonstração

$\Rightarrow$ Como $\phi(b) \in U_{s u b}$, $\phi(b)=\phi(A x) \leqq \sum_{j=1}^{q} x_{j} \phi\left(A^{j}\right) \leqq \sum_{j=1}^{q} x_{j} c_{j}=\langle c, x\rangle$

Sendo $\phi(b)=\langle c, x>$ vem que,

$$
\phi(A x)=\left\langle c_{g} x>e \sum_{j=1}^{q} x_{j} \phi\left(A^{j}\right)=\sum_{j=1}^{q} x_{j} c_{j}\right.
$$

$\Leftrightarrow)$ Trivial

\section{DEFINIÇÃO A2.12}

As condições i.) e ii.) do lema anterior são chamadas condições de folgas complementares para o par (PI), e seu dual geral.

Segue de folgas complementares que:

\section{TEOREMA A2.13}

Sejam $x \in X_{P I}$ e $\phi(.) \in U_{s u b \text {. }}$

Então, valendo as condiçöes de folgas complementares para o par $(x, \phi())$, tem-se que 
Apêndice 2: Dualidade Generalizada em Progrmação Inteira

$\mathrm{x}$ é solução otima de (PI) e

$\phi$ e solução ótima de seu dual geral

Demonstração

Basta observar que valendo as condiçôes de folgas complementares, $\phi(b)=<c, x>$ e do teorema fraco de dualidade segue a afirmação.

A validade dos resultados vistos se extende a problemas mais gerais onde as igualdades do problema são substituidas pela operação congruência modulo 1 , sendo que sua verificação nesse caso nao apresenta modificações significativas com relação ao que aqui fizemos. 


\section{REFERENNCIAS}

plications

[BM76] - Bondy, J. and Murty, U. S. R.- Graph Theory with Ap-

London, The Macmillan Press, 1976. 274p.

[BM83] - Bagchi, A and Mahanti, A - Search Algorithms under Different kinds of Heuristics : A Comparative Study,

Journal of the association Computing Machinery, 30(1): 1-21, 1983.

[BJ74] - Burdet, C. A. and Johnson, E. L.- A Subadditive Approach to the Group Problem of an Integer Programming .

Math. Programming Study 2(1974) 51-71

[BJ74] - Burdet, C. A. and Johnson, E. L.- A Subadditive Approach to solve Linear Integer Programming.

Annals of Discrete Mathematics 1, Studies in Integer Programming (North - Holland, Amsterdam, 1977 ) 117-144

[Cu79] - Cunningham, W. H. - Theoretical Proprerties of the Network Simplex Methods

Matematics of Operations Research, Vol 4, No 2, 1979 Network

[Da60] - Dantzig, G. B. - On the Shortest Route through a Road

Managment Science, 6, 2, 1960

[Di59] - Dijkstra, E.W - A Note on two Problems in Conexion with Graphs

Numerische Mathematik 1, 269-271 (1959)

[Dr68] - An Approach of some Shortest - path Algorithms

[Ge77] - Gelperin, D. - On the Optimality of $A^{*}$ Artificial Intelligence 8 (1) : 69-76, 1977 
[Go63] - Gomory, R. E. - An Algorithm for Integer Solutions to Linear Programs

In: R. L. Graves and P. Wolfe, eds, Recent Advances in Mathematical Programming (Mc Graw Hill, NY, 1963) 269-302

[Go65] - Gomory, R. E. - On the Relation between Integer and Non - integer Solutions to Linear Programs

Proc. Nat. Acad. Sci. U.S.A. 53(1965) 260-265

[GN72] - Garfinkel, R. S. and Nemhauser G.L - Integer Progamming New York, John Wiley, 1972.

\section{Linear Programs \\ [Go63] - Gomory, R. E. - An Algorithm for Integer Solutions to}

In: R. L. Graves and P. Wolfe, eds, Recent Advances in Mathematical Programming (Mc Graw Hill, NY, 1963 ) 269-302

[Go65] - Gomory, R. E. - On the Relation between Integer and Non - integer Solutions to Linear Programs

Proc. Nat. Acad. Sci. U.S.A. 53(1965) 260-265 [Go78] - Gonzaga, C - Busca de Caminhos em Grafos e Aplcações

I Reunião de Matemática Aplicada - Gávea, 1978

[Ha69] - Harary, F - Graph Theory

Addison Wesley, 1969. 274p.

[HH86] - Humes Jr., Carlos e Humes Ana Flora Pereira Castro. Progamação Linear : Um primeiro curso.

Rio de Janeiro, SBMAC, 1986. 213P.

[HNR68] - Hart, P. , Nilsson, N. and Raphael, B - A Formal Basis for the Heuristic Determination of Minimun Cost Paths

IEEE Trans. Systems, Man and Cybernet. 4(July 1968), 100-107

[Hu69] - Hu, T. C. - Integer Programming and Network Flows Addison Wesley, 1969. 452p. 

est Paths

[Jo73a] - Johnson, E.L - Cyclic Groups, Cutting Planes and ShortT.C.Hu and S. Robinson, Academic Press, New York, 1973. rithm [Jo73b] - Johnson, D. B. - A Note on Dijkstra shortest - path algo1973.

Journal of the association Computing Machinery, 20 385- 388 ,

[Jo79] - Johnson, E. J. : On the Group Problem and a Subadditive Approach to Integer Programming

Annals of Discrete Mathematics 5 (1979) 97-112

[Jo80a] - Johnson, E, L - Integer Progrmming : Facets, Subadditivity and Diality for Group and Semi-Group problems

[Jo80b] - Johnson, E. L - Subadditive Lifting Methods for Particioning and Knapsack Problems

Journal of Algorithms I, (1980) , 75- 96 matroids

[La.76] - Lawler, E - Combinatorial Optimizatin : networks and New York, Holt, 1976. Algorithms

[Ma77] - Martelli, A - On the Complexity of Admissible Search Artificial Intelligence 8(1):1-13, 1977

[Ni71] - Nilsson, N - Problem Solving Methods in Artificial Intelligence

Mc Graw Hill (NY), 1971

[Ni80] - Nilsson, N - Principles of Artificial Intelligence Mc Graw Hill (NY), 1971 lem Solving

[Pe83] - Pearl, J - Heuristics :Intelligent Search for Computer Prob- 
Addison Wesley Publishing Company, 1984

[Ro70] - Rockafellar, R. T. - Convex Analysis

Princeton University Press, Princeton, N.J., 1970

[Si66] - Simmonard, M - Linear Programming

Prentice - Hall , Englewood Cliffs, 1966

[Te82] - Terada, $R$ - Desenvolvimento de algoritmos e Complexidade de Computação

Terceira Escola de Computação, PUC - RJ, 1982.

Duality Theory in Mathematical Programming

[TW81] - Tind J and Wolsey, L - An Elementary Survey on General

Mathematical Programming, 21(1981) 241-261

[WH60] - Whiting, P. D. - A method for finding the Shortest Route through a Road Network Oper Res. Quat. 11, 37-40 (1960) 